Final Report

\title{
Natural Gas Vehicle Cylinder Safety, Training and Inspection Project
}

Cooperative Agreement: DE-FC26-05NT42608

Project Period: 10/1/05 - 12/31/08

January 30, 2009

Submitted to: National Energy Technology Laboratory US Department of Energy

Submitted by:

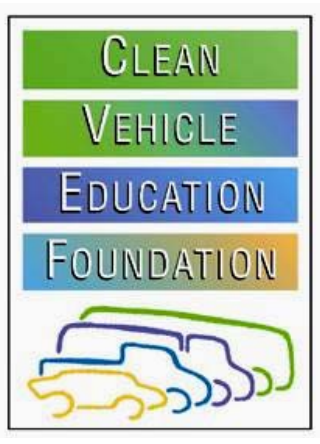

Clean Vehicle Education Foundation 6011 Fords Lake Ct.

Acworth, GA 30101

Author:

Hank Seiff

Director of Technology

703-534-6151

FAX: 703-534-6151

hseiff@cleanvehicle.org
Point of Contact:

Doug Horne

President

770-424-8575

FAX: 770-424-8575

dbhorne@cleanvehicle.org 


\section{DISCLAIMER}

This report was prepared as an account of work sponsored by an agency of the United States Government. Neither the United States Government nor any agency thereof, nor any of their employees, makes any warranty, express or implied, or assumes any legal liability or responsibility for the accuracy, completeness, or usefulness of any information, apparatus, product, or process disclosed, or represents that its use would not infringe privately owned rights. Reference herein to any specific commercial product, process, or service by trade name, trademark, manufacturer, or otherwise does not necessarily constitute or imply its endorsement, recommendation, or favoring by the United States Government or any agency thereof. The views and opinions of authors expressed herein do not necessarily state or reflect those of the United States Government or any agency thereof. 


\section{Table of Contents}

$\begin{array}{ll}\text { I Abstract } & 4\end{array}$

II Executive Summary $\quad 4$

III Project Tasks and Results $\quad 6$

A - Public and Industry Awareness Campaign 6

1 - Targeted outreach effort to CNG vehicle users and appropriate safety officials responsible for vehicle or infrastructure support $\quad 6$

2 - Emphasize need for vehicle/cylinder inspections and training

3 - Review cylinder inspection requirements and recommend best practices for fleet operators and public safety officials

4 - Recommend training practices to assure that cylinder inspectors are qualified and competent

5 - Recommend procedures to document and assure that cylinders are inspected

B - Training Scholarships and Tuition Assistance

1 - Identify Qualified Training Providers and Inspection Certification Programs

2 - Establish a scholarship or tuition assistance program to encourage vehicle users and safety officials to take the required training to become certified inspectors

3 - Inform industry of the DOE Cylinder Safety Project and the availability of training scholarships or tuition assistance

4 - Organize and operate the scholarship/tuition assistance program, and administer funding for scholarships (for both training and testing of students)

C - Evaluate Current Training and Testing Practices

1 - Work with individual fleets as well as training centers to determine if their present training program for cylinder safety is adequate and effective

2 - Recommend updates to training and testing programs where needed, and Provide guidance to training coordinators that outlines comprehensive NGV safety training recommendations, in addition to cylinder training (including where the training can be obtained)

D - CNG Cylinder Safety Monitoring and Investigation Activities

1 - Establish an industry incident reporting program 
2 - When necessary, participate in or conduct investigations of cylinder failures/incidents; document findings in lessons learned format

3 - Make recommendations for improvements in cylinder safety codes and standards, when appropriate

4 - Review and make recommendations for proper inspection procedures for stationary CNG storage cylinders

E - CNG Cylinder Recertification

1 - Review needs for procedures to address vehicles and cylinders that need to be in service for extended lifetimes

2 - Conduct a review of worldwide container standards related to extended service periods, accepted container lifetime, pertinent inspection and recertification procedures

3 - Make recommendations for extended lifetime container recertification procedures and safety testing

4 - Initiate appropriate standards updates to address recertification

F - Compressed Hydrogen/HCNG Cylinder Safety Considerations

1 - Evaluate synergies between CNG and compressed hydrogen and/or hydrogen/natural gas blend cylinders to determine similarities from a safety inspection and training perspective

2 - Recommend changes or improvements to CNG cylinder training and inspection guidelines to included expanded use of hydrogen (and HCNG blend) cylinder use in the future

IV Appendices

Appendix A - Summary of Communications Efforts

Appendix B - Recommended Periodic Motor Vehicle Inspection Requirements

Appendix C - New NFPA 52 Dispenser Wording and Fueling Station Hand Out

Appendix D - Summary of International Inspection and Recertification Activities

Appendix E - Review of Cylinder and CNG Fuel System Training Safety Requirements and Actual Practices

Appendix F - Scholarship Application Form

Appendix G - Sample of Correspondence with Scholarship Applicant Appendix H - Scholarship Record 
Appendix I - Fuel System Inspector Study Guide

Appendix J - Cylinder and Vehicle Manufacturers' Inspection Requirements

Appendix K - NGV Incidents Summary Document

Appendix L - Detailed File of NGV Incidents

Appendix M - Recommended Minimum In-Service Inspection of CNG Station

Storage Cylinders and Pressure Vessels

Appendix N - Summary of Recertification Results from Surveys

Appendix O - Procedure for Requalifying All-Steel NGV Cylinders

Appendix P - Some Things to be Learned from the "Other" Compressed Gas Fuel System 


\section{$\underline{\text { Abstract }}$}

Under the auspices of the National Energy Technology Laboratory and the US Department of Energy, the Clean Vehicle Education Foundation conducted a three-year program to increase the understanding of the safe and proper use and maintenance of vehicular compressed natural gas (CNG) fuel systems. High-pressure fuel systems require periodic inspection and maintenance to insure safe and proper operation. The project addressed the needs of CNG fuel containers (cylinders) and associated highpressure fuel system components related to existing law, codes and standards (C\&S), available training and inspection programs, and assured coordination among vehicle users, public safety officials, fueling station operators and training providers. The program included a public and industry awareness campaign, establishment and administration of a cylinder inspector certification training scholarship program, evaluation of current safety training and testing practices, monitoring and investigation of CNG vehicle incidents, evaluation of a cylinder recertification program and the migration of CNG vehicle safety knowledge to the nascent hydrogen vehicle community.

\section{Executive Summary}

A report by CVEF to the Department of Energy ${ }^{1}$ estimates there were 92,000 natural gas vehicles (NGV) in the United States in 2005. Worldwide, there are more than seven million NGVs ${ }^{2}$ with the numbers growing quickly in countries like Argentina, Brazil, Iran, Pakistan, India and China.

Although there are currently no commercially available hydrogen vehicles, government agencies, motor vehicle manufacturers and suppliers, the press and the general public are looking toward a "hydrogen future" which will include safe, low (or zero)-emitting, fuel-efficient fuel-cell motor vehicles. And between today's NGVs and tomorrow's hydrogen fuel-cell vehicles, there is increasing interest in internal-combustion engine (ICE) vehicles running on mixtures of natural gas and hydrogen (HCNG).

The major public safety concern with NGVs and hydrogen fueled vehicles is the gaseous fuel storage system. Most of today's NGVs store fuel in 3000 or 3600 psi containers, although discussion of compressed hydrogen storage for fuel cell vehicles currently ranges up to 10,000 psi. While storage of gas in high-pressure cylinders is normal procedure in the working world (e.g., medical gases in hospitals and welding gases on construction sites), it is not standard operating procedure in millions of private motor vehicles. Catastrophic failure of such a high-pressure vessel is a major safety concern.

Failure of high-pressure gas storage vessels in the working world is rare. ASME, and federal hazardous materials codes, standards and regulations are followed and en-

\footnotetext{
${ }^{1}$ Yborra, Stephen C., "Roadmap for Development of Natural Gas Vehicle Fueling Infrastructure And Analysis of Vehicular Natural Gas Consumption by Niche Sector," p 2, 10-2007

${ }^{2}$ International Association for Natural Gas Vehicles, http://www.iangv.org/
} 
forced. In the case of NGVs, high-pressure vehicular containers are built to Federal Motor Vehicle Safety Standard 304 (49 CFR 571.304) and the more stringent industry standard, ANSI NGV2. Both standards require the cylinders to be conspicuously labeled with the requirement that they be visually inspected by a qualified container inspector at least every 36 months, or at the time of any re-installation or fire, for external damage and deterioration.

The Clean Vehicle Education Foundation's predecessor organization established the task group to write the original NGV2 standard (used as the basis for FMVSS 304) and later brought together the National Alternative Fuels Training Consortium (NAFTC) and CSA America to provide training and certification testing for NGV cylinder inspectors. The training and certification program turns out qualified inspectors prepared to assure the safety of NGV cylinders in use.

There has been only one fatality in the US due to the failure of an NGV cylinder or fuel system. Yet CNG cylinder or high-pressure fuel system failures can be serious and can cause injuries and fatalities. Improper conversions to CNG, damage to uninspected fuel systems, failures in localized fires, and failures of pressure relief systems have led to accidents overseas and in the United States.

Despite the cautionary label, not all CNG cylinders in the US are periodically inspected. While vehicle owners are clearly aware of the need for brake or tire inspection, many NGV owners seem unaware of the need for CNG cylinder inspection. State safety inspection (in some states) insures that most vehicle systems are safe, but almost never concerns itself with NGV fuel systems.

Under the auspices of the National Energy Technology Laboratory and the US Department of Energy, the Clean Vehicle Education Foundation conducted a three-year program to increase understanding of the safe and proper use and maintenance of vehicular compressed natural gas (CNG) fuel systems. The project identified and addressed CNG fuel cylinders and associated high-pressure fuel system components needs related to existing law, codes and standards, available training and inspection programs, and assured coordination among vehicle users, public safety officials, fueling station operators and training providers. The program included a public and industry awareness campaign, the establishment and administration of a cylinder inspector certification training scholarship program, the evaluation of current safety training and testing practices, monitoring and investigation of CNG vehicle incidents, evaluation of a cylinder recertification program and the migration of CNG vehicle safety knowledge to the nascent hydrogen vehicle community.

Some highlights of the program include:

- A total of 69 press releases, articles, advertisements, and "other" communications were targeted to the NGV and other appropriate technical trade press. Our "CNG Cylinder Safety, Training and Inspection Program" web page (http://www.cleanvehicle.org/technology/cylinder.shtml) provides in one place a suc- 
cinct and specific summary of all the information on cylinder inspection requirements, inspectors, training and scholarships needed by NGV users.

- The approval of a new NFPA 52 CNG dispenser warning and fueling station handout emphasizing the need for CNG fuel system inspection

- The development of recommended periodic CNG motor vehicle inspection requirements.

- The implementation of a program which offered "scholarships" to pay for CNG fuel system inspector training and certification to 286 technicians.

- The revision and updating of the CSA cylinder inspector test procedure

- The production of a CNG Fuel System Inspector Study Guide

- The detailed investigation of two cylinder rupture incidents, collection of information on 29 incidents and the transfer of information gained toward improved C\&S.

- A recertification procedure for Type 1 all-steel cylinders

- The migration of CNG vehicle experience to hydrogen codes and standards including a technical paper delivered to an international cylinder safety conference

\section{Project Tasks and Results}

The object of this project was to increase the understanding of the safe and proper use and maintenance of vehicular compressed natural gas (CNG) fuel containers including installation of pressure relief devices and proper venting. These high-pressure fuel containers require periodic inspection and maintenance to insure safe and proper operation. This project identified and addressed CNG fuel system needs related to existing law, codes and standards, available training and inspection programs, and assured coordination among vehicle users, public safety officials, fueling station operators and training providers.

\section{A - Public and Industry Awareness Campaign}

The NGV trade press, NGV conferences, CVEF sponsored committees and affiliated Standards Development Organizations (SDOs) (e.g., CSA, NFPA, SAE) were used to "get the word out" on the importance of cylinder inspection and the availability of training and certification testing scholarships. We used our technical expertise and involvement in the industry to review the requirements and training and testing procedures in place against their effectiveness in service and recommend improvements as needed. We developed a periodic motor vehicle inspection procedure for CNG vehicles and requested AAMVA (American Association of Motor Vehicle Administrators) and CVSA (Commercial Vehicle Safety Alliance) to include it in their inspection procedures to increase the likelihood that cylinders are inspected.

1 - Targeted outreach effort to CNG vehicle users and appropriate safety officials responsible for vehicle or infrastructure support -

- A total of three press releases, 29 articles, 12 paid advertisements, 17 "other" advertisements (generally paid for by other organizations, such as CSA), and eight "other" communications vehicles were targeted to the NGV and other appropriate technical trade press. Our "CNG Cylinder Safety, Training and Inspection Program" web page 
(http://www.cleanvehicle.org/technology/cylinder.shtml) provides in one place a succinct and specific summary of all the information needed by NGV users on cylinder inspection requirements, inspectors, training and scholarships.

- Discussions, presentations and exhibits stressing the need for cylinder and total NGV safety were presented at NGV trade shows and meetings.

- We worked with CVEF and NGVAmerica to reach vehicle manufacturers, system installers, parts suppliers and others to present the message of cylinder, fuel system and vehicle safety through articles, advertisements, press releases, meetings, exhibits, etc.

- We informed CVEF's Utility and Public Fleet Council and the Natural Gas Transit (and school bus) Users Group (TUG) of the need for cylinder and vehicle safety, including holding a special TUG meeting in Los Angeles for the sole purpose of providing "free" training and certification testing of cylinder inspectors.

- We worked with safety and technical officials through CSA, NFPA, SAE, AAMVA, etc., to improve codes and standards for the safety of vehicle fuel systems, improve NGV safety inspections, and inform NGV users of the need for cylinder inspection.

- We were unable to make direct contact with all individual CNG vehicle users as it is impossible to identify them all through state or federal records or the "Natural Gas Vehicle Road Map and Data Collection Effort." However we believe that most NGV fleets and many individual users were impacted by our communications efforts

- We effected a change in NFPA 52 (2010 edition) to add a notice indicating the need for periodic cylinder inspection to CNG dispensers.
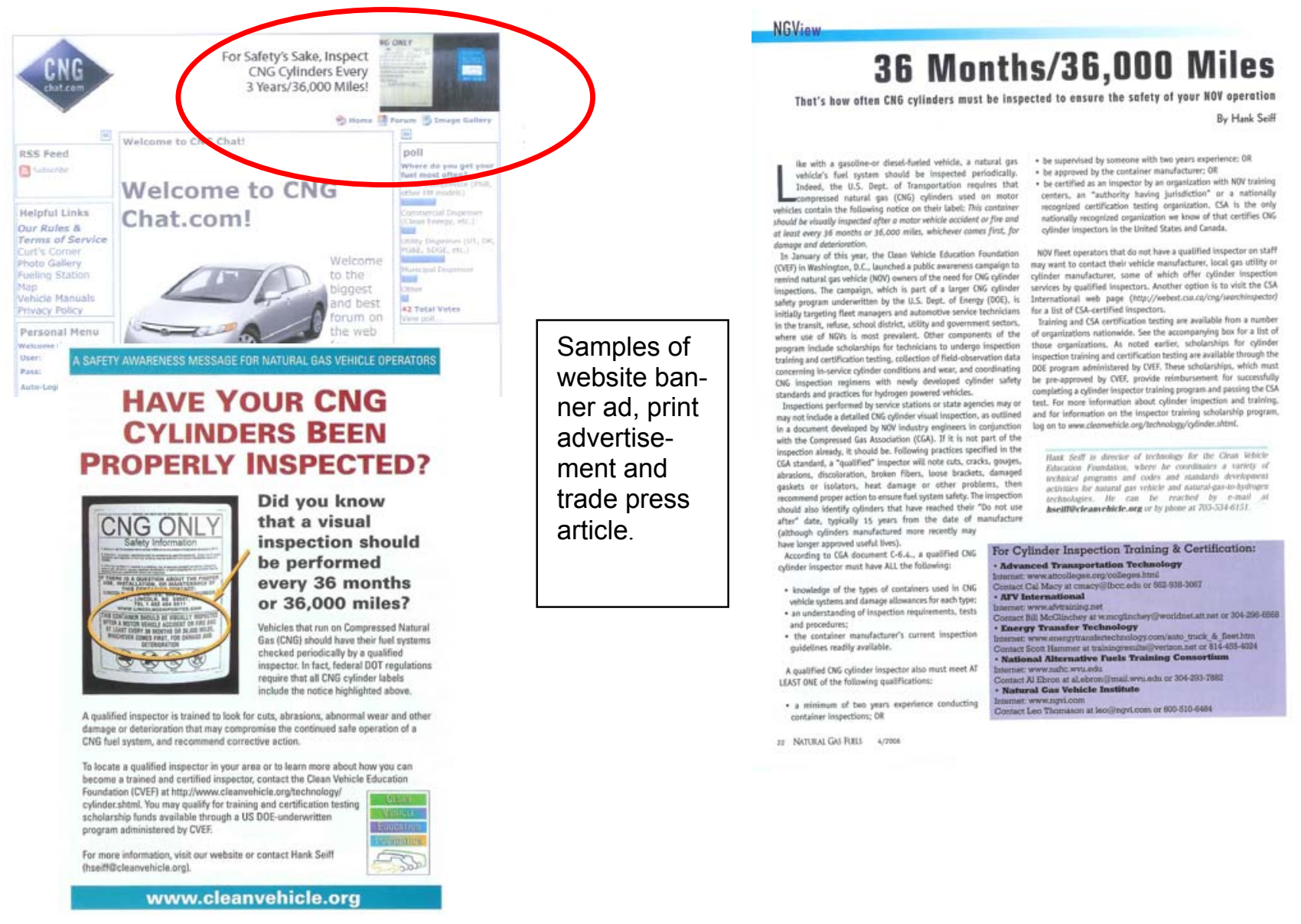
See Appendix A for a summary of communications activities under this project. See Appendix B for recommended periodic motor vehicle inspection requirements See Appendix C for new NFPA 52 dispenser wording and fueling station hand out.

2 - Emphasize need for vehicle/cylinder inspections and training - The targeted outreach program outlined above emphasized the need for vehicle/cylinder safety inspections and training, provided information on available training resources and where qualified inspectors may be found, and announced the availability of training and certification testing scholarships. The media for the message included published hard copy and electronic advertisements/notices, PowerPoint presentations, email messages, fliers/pamphlets, exhibits, training sessions, etc. It became clear however, that many individual NGV owners are not "getting the message" of the need for cylinder inspection. The addition of notices on CNG dispensers, the effort to have CNG inspection items added to state and commercial vehicle inspections, and a banner advertisement (through the end of 2008) on the preeminent CNG web site (www.cngchat.com) may help in this area, although it appears impossible to have universal cylinder inspection without state or national regulation.

3 -Review cylinder inspection requirements and recommend best practices for fleet operators and public safety officials - We reviewed the cylinder inspection (and recertification) requirements for Canada, Japan, Italy, Argentina, France, Brazil and Pakistan as input into our codes and standards, cylinder and vehicle inspection, and recertification activities. See Appendix D for a summary of international inspection and recertification requirements and activities.

4 - Recommend training practices to assure that cylinder inspectors are qualified and competent

- We reviewed Cylinder and CNG fuel system training safety requirements and actual practices, both as required by standards (e.g., NGV2, NFPA 52) and in actual use by front-line inspectors, by

- sending three project partners to separate cylinder inspector training programs,

- questioning 31 fleets on their cylinder inspection and safety practices, and

- visiting, in person or by phone, 14 fleets to determine their cylinder inspection and safety practices (See Appendix $E$ for a list of fleets reviewed for cylinder and CNG fuel system training safety requirements and actual practices)

- We considered suggestions from the three project partners who had experienced cylinder inspector training courses. And, working through CSA, the inspector certification agency, suggestions and recommendations from trainers, trainees and others were considered to improve the inspection, training and certification process.

- Information gained from these interactions was directly involved in the recommendation of changes to appropriate CNG and hydrogen C\&S groups

- We worked with CSA to revise and update the CNG fuel system inspection certification test

- We developed a CNG Fuel System Inspector Study Guide (See Appendix I) 
5 - Recommend procedures to document and assure that cylinders are inspected - CVEF developed new wording for NFPA 52's CNG dispenser warning sign, adding the text:: "Natural Gas Vehicle Fuel Cylinders Should be Periodically Inspected (Normally Every 3 Years) to Ensure Safe Operation of the Vehicle. Contact Vehicle or Cylinder Manufacturer". The new wording was accepted for the upcoming 2010 version of NFPA 52 with an explanatory flyer to be handed out by dispensing facilities. See Appendix $C$ for wording and flyer.

With the cooperation of CSA's NGV2 (CNG Containers) Technical Advisory Group, we developed a simple inspection procedure to be used by state Periodic Motor Vehicle Inspection agencies and the Commercial Vehicle Safety Alliance in their inspection of commercial vehicles per the Federal Motor Carrier Safety Regulations. See Appendix $B$ for the procedure. We submitted the inspection procedure to the American Association of Motor Vehicle Administrators for inclusion in their state inspection handbook and to the Commercial Vehicle Safety Alliance for inclusion in their inspection and out-of-service procedure.

AAMVA informed us that they would consider our request when the handbook is updated, but due to decreasing state interest in periodic motor vehicle safety inspection, they do not know when and if the handbook would be updated.

CVSA agreed with our recommendations but informed us they could not enforce them to put vehicles out of service unless the Federal Motor Carrier Safety Regulations were amended to require cylinder inspection per the FMVSS 304 label. On their recommendation we petitioned the Federal Motor Carrier Safety Administration of DOT in November 2008 to so amend their regulations. We have not heard back from them but expect the procedure, if successful, to take a number of years. CVSA is also considering whether to join our petition to FMCSA.

Despite these actions, the effort to inform each and every owner and operator of a CNG vehicle that cylinders should be inspected for safety at regular intervals has proved frustrating. Unfortunately there is no practical way to identify every owner/user of CNG vehicles. Most states do not keep vehicle records by fuel type (other than "diesel," "gasoline," and "other"). While vehicle OEM VINs identify fuel type, many or most CNG vehicles are converted after manufacture so their VINs would identify the original fuel. And, more important, while OEMs have records of the original purchaser of a vehicle by VIN, many/most CNG vehicles are no longer in the hands of their original owner.

It is "relatively" easy to identify and target fleets of CNG vehicles, and we have made every effort to do so through publications which are read by alternative fuel fleet owners.

And, of course, even though individual CNG vehicle owners, or fleets, may know that cylinders SHOULD be periodically inspected, that knowledge does not insure that they WILL be inspected. We know this is the case from a number of incidents which have occurred during the period of this contract. In one case a major airport shuttle fleet suffered a fatal cylinder rupture. During the investigation we found not only that the ruptured cylinder may not have been properly inspected after an accident, but that other cylinders added onto the vehicle to improve range had defects which should have been 
found during inspection. In another cylinder rupture case, a properly-inspected CNG vehicle was sold by a utility to one of its CNG technicians, who then did not re-inspect the cylinder after three years.

The US has one of the best motor vehicle safety records in the world. Because of this and because of Americans' strong love of individual freedom and negative attitude toward government regulations, we have relatively few controls to assure motor vehicle safety. For example only 18 states (and the District of Columbia and Puerto Rico) presently have periodic motor vehicle inspection (PMVI) programs (ref. AAMVA). Others have random inspections (e.g., CA) or require inspection when a vehicle is bought or sold (e.g., MD). Other areas of the country do required emission inspections but not safety inspections.

To try to "get the message" of cylinder inspection to all CNG vehicle users, we performed two "outside the box" projects as part of this contract (dispenser notices and $\mathrm{PMVI}$ ). As discussed above, we have succeeded in both cases, yet that will not ensure that all cylinders in use are given a proper safety inspection. Although this is frustrating, the same situation exists with other motor vehicle safety components, such as brakes, lights and tires. Without state or federal regulations requiring these inspections, we do not believe that it is possible to assure $100 \%$ safety inspection of cylinders or any other motor vehicle safety items. This may become an even more important issue if and when hydrogen vehicles gain widespread acceptance.

\section{B - Training Scholarships and Tuition Assistance}

A major assumption of this entire project is that the CNG fuel systems and especially CNG cylinders need to be periodically inspected per the requirements of their FMVSS 304 label (and ANSI NGV2) to assure the safety of operation of CNG vehicles. Although there are a number of ways one can become a "qualified" inspector per CGA C6.4 (the CNG and hydrogen cylinder inspection standard), the major route is to be certified as an inspector by a nationally recognized certification testing organization. The only such organization for CNG vehicular cylinder inspection in the US at the present time is CSA America. To encourage the proliferation of CSA certified inspectors, CVEF established a program of scholarships and tuition assistance to help potential inspectors train for and pass the CSA certification test.

1. Identify Qualified Training Providers and Inspection Certification Programs By virtue of having originated the inspector certification program in 1999 by working with the National Alternative Fuels Training Consortium at West Virginia University and CSA America, CVEF was already familiar and working with CSA and most providers of training for the CSA exam. Through our contacts in the NGV industry we were able to locate and speak to all those providing such training early on in the program. A list of these training providers is included on our web page discussing the training program: http://www.cleanvehicle.org/technology/cylinder.shtml.

2. Establish a scholarship or tuition assistance program to encourage vehicle users and safety officials to take the required training to become certified inspectors CVEF established a scholarship or tuition assistance program and widely "advertised " 
its availability throughout the NGV community (see phase I above). Also see http://www.cleanvehicle.org/technology/cylinder.shtml under "Is Scholarship Funding Available?" With the approval of NETL's project officer eligibility rules that were fair and acceptable to all were developed. A copy of the application form can be found in Appendix $\mathrm{F}$.

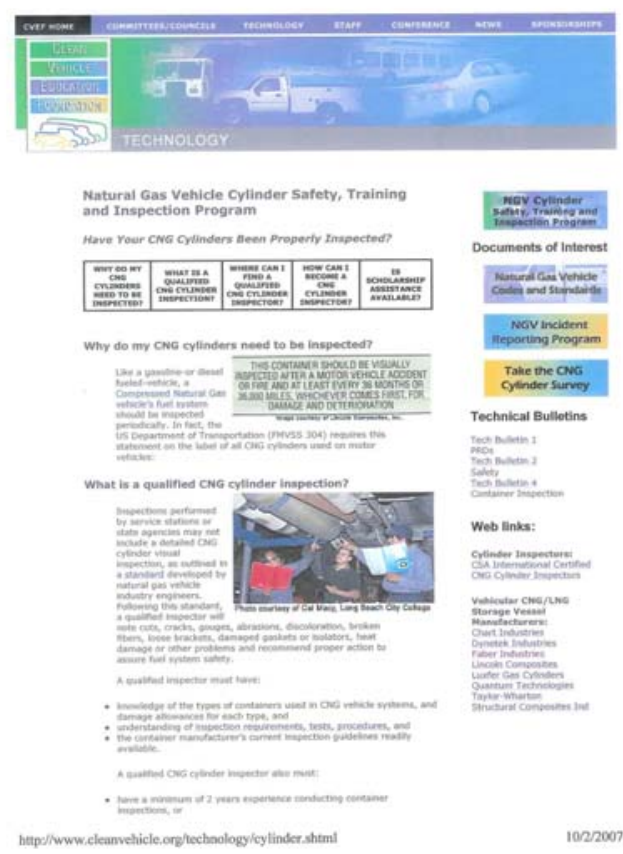

Cylinder Program web page.

3. Inform industry of the DOE Cylinder Safety Project and the availability of training scholarships or tuition assistance - In coordination with the Public and Industry Awareness Campaign, CVEF informed the NGV industry of the existence of the program, its content, and particularly the availability of scholarship funds as described in section III A 1 above. When one "Googles" "CNG Cylinder Inspection," they will find that the web page we produced on the program, http://www.cleanvehicle.org/technology/cylinder.shtml, comes up number one!

4. Organize and operate the scholarship/tuition assistance program, and - Administer funding for scholarships (for both training and testing of students) - After discussion among program participants and with the involvement of NETL's Project Officer, we developed a uniform scholarship application form (see Appendix F). Once applications were received (electronically in most cases, but some via FAX and some via post), we normally responded within a few days. Copies were kept of all correspondence with each applicant. Appendix $G$ is an example of correspondence with an applicant who was granted a scholarship but never sent in his "paperwork" to get his money and did not respond to our "reminder" emails.

We originally anticipated that 250 full-tuition scholarships (or equivalent, e.g., bringing the training to an organization's place of business) for training and certification testing would be provided during the first year of the program with 150 each for years two and three. As it turned out only 142 viable (of 197 total) applications were submitted and granted in the first year and only 88 persons actually completed coursework, 
passed the test and were reimbursed. Applications not considered viable included those missing proper information, those cancelled by the applicant, those from applicants outside the US, and those from major organizations well able to afford training on their own. In all cases we got back to applicants in a timely manner (within a few days in almost all cases) and explained the reason for granting or denying their application.

We are not sure of the reasons why only 88 persons actually received funding, compared with 142 whose applications were accepted, since most of those people simply never sent in the required "paperwork" for reimbursement, despite email reminders. We expect some failed the test, some never took the training and test and some just never submitted the paperwork. CSA provided us information on those who were granted scholarships during the second year and in that case $63 \%$ passed the test, $13 \%$ failed, and there was no record of $24 \%$ (we assume they never took the test). We assume similar percentages applied for the first and third years of the program.

One hundred and twenty-five scholarships were anticipated for the second and third years of the project, however a total of only 82 were approved in the second year (of 143 applications) and 62 approved of 91 applicants in the third year. In the second year 47 of the 82 scholarship recipients actually claimed their scholarship: in the third year 34 of 62 claimed their money.

In the second year, as in the first, the actual number of scholarships granted was limited by the smaller than expected number of applicants. So, in revising the third year's budget, we decreased the number of scholarships budgeted for and budgeted remaining money toward codes and standards work. But with a tremendous interest in NGVs (especially conversions) brought on by very high petroleum prices, we received far more applicants than expected. We were able to reprogram some money to cover this but finally ran out of funding at the end of June.

After receiving the necessary paperwork from scholarship recipients, we sent out scholarship checks within a few days. This ability to send out checks in such a timely fashion was in no small part due to NETL's easy and swift disbursement of funds through its electronic system.

Appendix $\mathrm{H}$ provides a detailed record of all scholarship applicants and the action taken on each application.

\section{$\underline{\text { C - Evaluate Current Training and Testing Practices }}$}

A training and testing program needs constant oversight and updating to assure that it meets the needs of safety and efficiency. In this task we worked to assure that the CNG fuel system training and testing certification program is training and testing for the right things and, most important, that technicians are performing adequate inspections.

1. Work with individual fleets as well as training centers to determine if their present training program for cylinder safety is adequate and effective - Because of stringent OMB survey requirements, our efforts to survey the NGV industry on the adequacy and effectiveness of the present cylinder safety training and certification program was somewhat limited. We did however discuss the program with CVEF's Utility and Public Fleet Council and the Transit Users Group and an informal survey was placed on our website. 
As discussed in section A 4 above, 31 fleets filled out our informal survey and 14 fleets were "visited," either by telephone or in person, to evaluate their CNG fuel system safety training and inspection program. A list of these fleets is found in Appendix E. The information from the surveys and visits was used by CVEF and its program partners in working with CSA to update and improve its certification testing procedures, with the assumption that the content of the test drives the training programs which prepare students for certification.

CVEF's partners, John Dimmick (John Dimmick LLC), and Murray Pennington and Rob Adams (Marathon Technologies), experts on high-pressure cylinders in CNG vehicles, attended training and testing sessions conducted by three different training centers and became certified inspectors. They were used by CVEF to contact the 14 fleets mentioned above. And, during the early parts of the program we were careful to keep major cylinder manufacturers apprised or our efforts and request relevant feedback. When we received relatively little initial feedback from the cylinder manufacturers, we decreased the frequency of our contacts with them,

2. Recommend updates to training and testing programs where needed, and Provide guidance to training coordinators that outlines comprehensive NGV safety training recommendations, in addition to cylinder training (including where the training can be obtained) - Based on the above evaluation of the adequacy and effectiveness of training and testing systems presently in place, we had planned to prepare a detailed report for DOE, providing information on the evaluation we have done and the results found and present recommendations for updates to training and test programs from that report to CSA and cylinder (fuel system) inspection trainers. However, during the second year of the program CSA decided to make major improvements to its CNG Fuel System Inspector Program by following the International Organization for Standardization, (ISO) 17024 Conformity Assessment standard, the global benchmark for personnel certification programs. They led an effort to update and improve the test itself, changed to a three-year re-examination cycle, and make other changes and improvements.

Rather than preparing a report for DOE, CSA, and CNG fuel system inspection trainers, we participated in the CSA certification test upgrade program by providing four members of our team to take part in in-person and telephone meetings, allowing us to impart our expertise directly into the test revision process. Our logic was that the certification test content directly drives the type and quality of training that is provided by the various training organizations. After the new CNG Fuel System Inspector Certification Program was announced by CSA America in July of 2008, we prepared a Fuel System Inspector Study Guide which we have provided to CSA and the various training organizations for their use. (See Appendix I).

CNG fuel system inspectors are primarily guided by the recommendations of the cylinder or vehicle manufacturer. They look to CGA pamphlet C-6.4 for general inspection requirements or if cylinder and vehicle manufacturer guidance is not available. As our work with CSA to improve the certification test and training progressed, we realized there was no single source of all available cylinder and vehicle manufacturer inspection information. So we collected all available information as part of this program. That information, in electronic format, has been provided to CSA to make available to their certified inspectors, insofar as their legal council will permit. We have also sent copies to 
trainers and inspectors as they have requested it. A copy is included in this report as Appendix J.

$\underline{D}$ - CNG Cylinder Safety Monitoring and Investigation Activities

Industry codes and standards (C\&S) insure that compressed natural gas vehicles are built and maintained to high levels of safety. Information gained from evaluating "incidents" involving CNG vehicles must be considered in improving these codes and standards. By taking part in the writing and revising of C\&S CVEF insures that the experience of its staff and partners, including that gained through the study of NGV incidents and the other tasks of this program, is brought to bear on maintaining and improving the safety of CNG vehicles in the US.

1- Establish an industry incident reporting program - CVEF (and NGVC before it) have been collecting information on natural gas vehicle "incidents" as long as they have been in existence. The NGV industry has long been aware that we collect incident information in an informal program to improve NGV safety and has consequently provided us with the needed information. Under the DOE/NETL program we investigated the possibility of "formalizing" our incident reporting program. To do this we reviewed similar programs conducted by the Department of Transportation's Pipeline and Hazardous Materials Safety Administration (PHMSA), the Compressed Gas Association (CGA) and the Institute of Makers of Explosives (IME).

When we contacted them, CGA had recently discontinued their incident reporting program because of legal issues. IME's members are used to being regulated so are not uncomfortable reporting incidents to their trade association. However the information is protected in the sense that it is not disclosed outside IME, yet we were told that companies still routinely withhold information based on the advice of counsel. PHMSA's hazardous materials reporting program is, of course, enforced by government regulation.

Given the motor vehicle industry's relatively unregulated environment and the fact that our incident files are open to all, at least within the industry, it was evident that only an informal incident reporting program was appropriate. CVEF has no regulatory authority nor was there any likelihood that any regulatory authority for a CNG vehicle incident reporting program would be considered. We therefore decided to continue the program which had been in place of years, but reminded the industry of the need to submit incident information through a press release, information on our website (http://www.cleanvehicle.org/IncidentReportingNoticeforWeb.pdf) and an article in the NGVAmerica newsletter.

Under this program we digitized the information on all incidents we have collected over the years. Both a short summary of each NGV incident in a "Word" document and details from our files on each incident are available, as appropriate, to those wishing to improve the safety of the industry. We have included a copy of the "Word" summary document as Appendix $\mathrm{K}$ and the detailed file as Appendix L.

Whereas we had collected detailed information on 56 incidents previous to this program, our files now have detailed information on 85 incidents through the end of 2008. 
We have also begun putting together a list of NGV incidents internationally, for the benefit of the NGV industry worldwide. That list can be found at HTTP://cleanvehicle.org/NGVIncidents. To access it the username is "NGVINC" and the password is "CVEF-1998."

2- When necessary, participate in or conduct investigations of cylinder failures/incidents; document findings in lessons learned format - Years ago, GRI (Gas Research Institute, now part of GTI, Gas Technology Institute) had funding available to conduct investigations of NGV incidents, when called upon. Under this program NETL/DOE provided funding to conduct or help conduct detailed investigations of one or two CNG vehicle incidents each year.

To most effectively use our ability to investigate incidents, we looked for incidents where the CNG fuel system did not perform as expected or where there was serious damage or injury or fatality. However, in many such cases, the vehicle owner or manufacturer, government agency, involved fleet, fueling station owner, etc., or their insurance company takes control of incident investigation and provides only limited data to CVEF and to the public.

During the first year of the program, we were made aware of no incidents in the US where our information investigation criteria were met.

During the second year of the program a serious fire-related incident took place when an arsonist torched 12 vehicles in a Seattle, WA city vehicle yard, including one Honda CNG car. The fuel tank ruptured, apparently due to localized flame impingement away from the PRD. This incident was investigated by the Seattle Fire Department and the incident led to a recall of Honda GX CNG vehicles and the installation of a fireproof blanket behind the rear seat of the vehicle. Later in the year, in Carson, CA, a Comdyne add-on tank at the rear of a Ford E350 SuperShuttle van ruptured during the first fueling after the repair of a rear-end accident. This was the first known US incident where a failure of the natural gas fuel system contributed to a fatality. CVEF assisted the California Department of Occupational Safety and Health (DOSH) in investigating this incident, which turned out to have been caused by stress corrosion cracking of the fiberglass cylinder over-wrap which was in turn caused by the exposure of the CNG cylinder to car battery acid when it was rear-ended in the earlier accident three weeks prior to the tank failure. A copy of the report on the incident can be found at http://seal.onlinelrms.net/rv.asp? rn=420-3B43GN\&rp=5442.

After completion of the investigation, the results were announced in articles in the weekly NGVAmerica newsletter, and a press release was issued and webpage (http://www.cleanvehicle.org/technology/Comdyne Warning.pdf) established warning the industry of potentially catastrophic results if Comdyne cylinders were exposed to battery acid. No additional action was taken since most of the Comdyne cylinders manufactured were no longer in service and the cylinder manufacturer had gone out of business.

During the third year of the contact there were three incidents where we volunteered our assistance in conducting an investigation in hopes of developing information which could help improve the safety of NGVs. In the first case a Comdyne cylinder on a privately-owned 1995 or 1996 Dodge van ruptured during fueling in Grand Rapids, MI with minor injuries to driver/fueler. We were told that this was probably caused by 
gouges/damage to the fiberglass wrapping and that the cylinders had not been inspected in at least four years. However neither the utility that sold the vehicle to the private owner (an ex employee) nor the owner agreed to our involvement in any investigation.

On June 9, 2008 a Type 2 Lucas cylinder on a 1998 Ford E350 van owned by Philadelphia Gas Works ruptured at a PGW fueling station in a Philadelphia suburb. We worked with PGW and NHTSA to investigate this incident and found that the rupture was due to a combination of stress corrosion cracking of the fiberglass cylinder overwrap along with likely overpressurization of the cylinder during fueling. Unfortunately, the exact cause of the stress corrosion cracking was not pinpointed in laboratory tests. In addition, although our investigation found evidence that the CNG dispensers in use could dispense gas at higher than acceptable pressures, there was no direct evidence that a single incidence of very high pressure caused the rupture, although indirect evidence pointed to that possibility.

The final reports and other information on the two incidents investigated in 2007 and 2008 can be found in Appendix L - Detailed File of NGV Incidents (note that this appendix and appendix $\mathrm{J}$ are not actually included in this report since, in order to meet NETL's requirements that the final report accompanying form 241.3 be uploaded as one document in pdf file, these appendices would be unacceptably compromised [all files in the appendix files are listed by Adobe in alphabetical order, rather than under appropriate subfolders]. Please contact CVEF for a CD copy of the Detailed File of NGV Incidents or these specific investigation reports.)

A third incident of interest also occurred in 2008, in which a cylinder ruptured on a 1995 Chrysler Corporation van in Corona, CA July 16, injuring five people. The van, which was privately owned, was fueling at a City of Corona fueling station. Although we volunteered to help in an investigation, the City Attorney refused our offer and also refused to provide information on a laboratory evaluation of the ruptured cylinder which Corona had performed.

3 - Make recommendations for improvements in cylinder safety codes and standards, when appropriate - Relevant information gained from the incident reporting program and the investigations discussed above is used directly by CVEF to make recommendations for improvements in appropriate NGV-related safety C\&S. In fact, at the beginning of most CSA NGV standards meetings a time is set aside to review recent incidents and discuss any relevant lessons for the standards under consideration.

We did not keep a formal list of recommendations made based on incident experience during this program, but a few samples are:

- Discussions were initiated in the PRD1 and NGV2 technical advisory groups based on the cylinder rupture caused by localized flame impingement during the Seattle Honda incident. This and other incidents (such as a similar case during a Ford Crown Victoria fire in Madison, WI in 2002) indicate that more stringent tests for cylinder protection from localized fire are needed in one of these standards.

- The failures of at least two tanks in the past few years, caused by chemical induced stress corrosion cracking, indicates a potential need to restrict fiber cylinder over-wrap materials or strengthen the environmental test in NGV2. 
This issue has and will continue to be raised to the NGV2 Technical Advisory Group.

- The report on the likely overpressurization of the tank in the PGW incident includes a recommendation that NFPA 52 specifically require redundant pressure control strategies on CNG dispensers.

4. Review and make recommendations for proper inspection procedures for stationary CNG storage cylinders - the majority of stationary fueling station CNG containers are built to ASME requirements. This is because of the high safety factor and general confidence in ASME pressure vessels and also apparently because only visual inspection is required by ASME. A few years ago CVEF researched the inspection requirements for DOT 3AA storage cylinders, which are used in some fueling stations. In response to an inquiry, OSHA responded that DOT's five-year retest, including a visual internal and external examination and a hydrostatic pressure test were applicable, despite the fact that these cylinders are not subject to DOT jurisdiction since they are not used in interstate transportation. Based on the best available information, CVEF has prepared a Recommended Minimum In-Service Inspection of CNG Station Storage Cylinders and Pressure Vessels report which is found in Appendix M.

Because of a lack of interest among CNG fueling station owners and operators, we did not go ahead and ask the NGV2 TAG or the NFPA 52 committee to consider adding this document as an informative index to their standards.

\section{E-CNG Cylinder Recertification}

All CNG cylinders built to meet the original (1992) version of NGV2 were designed and marked for a service life of 15 years. It was only with the 1998 version that a 20-year life was permitted and the 2007 version that a 25 -year life is allowed. Some of us, the author included, have assumed that the occasional call from a school bus or other NGV operator for a method of extending cylinder useful life indicates a growing need for a recertification process. We suspect that some cylinders are being destroyed after their expiration date despite having many potentially useful years of life left. This unnecessarily increases costs to present and potential users of NGVs. With these concerns in mind we explored the possibility of developing a process by which nominally expired cylinders could be recertified/requalified.

1. Review needs for procedures to address vehicles and cylinders that need to be in service for extended lifetimes - Because of stringent OMB survey requirements, our efforts to survey the NGV industry on the adequacy and effectiveness of the present cylinder safety training and certification program was somewhat limited.

As can be seen in the summary of recertification results from the surveys in Appendix $\mathrm{N}$, there was by no means overwhelming support for a need for a recertification procedure. In fact, only $37 \%$ of the respondents felt that the fact that most CNG cylinders have a design life of 15 years (and should be replaced after that time) caused a problem for their fleet. Although only 11 of the 30 respondents felt there was a problem, 17 felt it would be useful to have a method of "recertifying" cylinders so that they could be used for an additional period of time. 
Certainly the small size of the survey cannot be projected to represent the universe of CNG vehicle users. However we judged there was sufficient interest to justify continuing to review the potential for developing a cylinder recertification process in the US.

2. Conduct a review of worldwide container standards related to extended service periods, accepted container lifetime, pertinent inspection and recertification procedures - There is no need to "reinvent the wheel." Other countries have far more NGVs in service than the US and have different standards for cylinder construction, use and inspection. CVEF and its partners reviewed the practices of seven foreign countries (Canada, Japan, Italy, Argentina, France, Brail and Pakistan) to determine whether there was a consensus method of safely continuing the use of CNG cylinders beyond their originally designed life. The results of that review are summarized in Appendix D.

In summary, there is no standard lifetime for a CNG cylinder and, of the countries surveyed, only Canada has in place a system for recertification after a cylinder's original lifetime is completed, and that procedure is only for type 1 all-steel cylinders.

3. Make recommendations for extended lifetime container recertification procedures and safety testing - Based on the Canadian standard, a requalification procedure was devised for type 1 all-steel cylinders and accepted by the NGV2 Technical Advisory Group to become an informative index in the upcoming version of NGV2. That procedure is included as Appendix $O$. It should be noted that this procedure will not be of significant assistance in lengthening the life of many CNG cylinders used in the US, since most US CNG vehicles use other than type 1 steel cylinders. However it was felt that the acceptance of this procedure was an important first step, a "place holder," which will allow the US industry to consider whether a recertification procedure for other types of cylinders should be considered in the future.

4. Initiate appropriate standards updates to address recertification - As section $E$ 3 above describes, a recertification procedure was accepted by the NGV2 Technical Advisory Group as an informative appendix for the upcoming version of the standard. Since it is more of a "place holder" than a realistic method of recertifying a significant number of CNG cylinders in vehicular use in the US, it was not felt necessary to recommend it's adoption by the NFPA 52 committee, or NHTSA for revision of FMVSS 304.

\section{$\underline{\text { F - Compressed Hydrogen/HCNG Cylinder Safety Considerations }}$}

A major advantage of this Natural Gas Vehicle Cylinder Safety, Training and Inspection Project has been the ability to transfer decades of knowledge about compressed natural gas as a vehicle fuel to the nascent hydrogen vehicle industry. This was done primarily by taking part in hydrogen and HCNG codes and standards meeting but also by writing and delivering a paper addressing some of the major issues which CNG vehicle experience shows need to be considered by hydrogen vehicle manufacturers and users. 
1. Evaluate synergies between CNG and compressed hydrogen and/or hydrogen/natural gas blend cylinders to determine similarities from a safety inspection and training perspective - From CVEF's work with various standards development organizations (SDOs) involved in NGV codes and standards, it was immediately obvious that NGV C\&S were not necessarily written to incorporate hydrogen and HCNG vehicles and needed change. Most of these SDOs early-on determined either to widen the codes and standards in place (e.g., NFPA 52 and CGA C-6.4) or develop parallel C\&S for hydrogen and/or HCNG based on the natural gas documents in place (e.g., CSA NGV and PRD documents).

Although it seems obvious that there are many synergies between the two flammable compressed gases, there are also some differences. For example, while CNG is stored in 3000 and 3600 psi cylinders, hydrogen has a much lower energy density and, in order to have adequate fuel energy available, cylinder pressure in the 5000 to 10,000 psi range are being considered for hydrogen. In addition, hydrogen can cause hydrogen embrittlement, a potentially serious safety issue, to types of steel cylinders and appurtenances which are perfectly safe for compressed natural gas.

2. Recommend changes or improvements to CNG cylinder training and inspection guidelines to include expanded use of hydrogen (and HCNG blend) cylinder use in the future - Members of the CVEF team served on codes and standards development committee of CSA, SAE, NFPA and other SDOs. CSA's NGV standards technical advisory groups considered whether to simply add hydrogen to the extant NGV standards but decided to develop parallel standards instead. For example, the extant NGV2 CNG cylinder standard was used as a basis for the development of HGV2 for hydrogen cylinders and PRD1 (pressure relief devices) served as the basis for HPRD1 for hydrogen PRDs. In most cases the same people (including CVEF staff and partners) served on both groups,

NFPA decided to widen its NFPA 52 standard which originally covered only CNG and $L N G$ vehicles and infrastructure to include hydrogen vehicles and fueling stations. CGA in C-6.4 (fuel system inspection) did the same. SAE, which had few recommended practices covering CNG vehicles, is developing a whole new set of RPs for hydrogen vehicles.

Although the issue has been discussed at some length, it is still not clear whether a separate set of C\&S will be written for HCNG or whether HCNG components and vehicles will be required to meet both $C N G$ and compressed hydrogen codes in place. In the meantime, some documents (i.e., NGV2 and NFPA 52) have been modified to make it clearer that they do not apply to HCNG mixtures with over $2 \%$ hydrogen.

In all cases, CVEF and its partners provided input gained from years or decades of experience in the natural gas vehicle industry.

CVEF's program manager wrote a technical paper entitled Some Things to be Learned from the "Other" Compressed Gas Fuel System and delivered it at the Critical CNG \& Hydrogen Cylinder Issues Workshop in Brussels, Belgium in March 2008. It explored "a few of the things to be learned from CNG vehicle history that can help assure the safety of compressed hydrogen tanks and fuel systems," such as the need for periodic cylinder inspection, the concern that PRDs can leak and, in present usage, may not protect against localized fires. An article about the paper published in NGVAmerica's 
newsletter offered copies to all interested persons. A copy of the paper is included as Appendix P. 
Appendix A - CNG Cylinder Program Communications Record

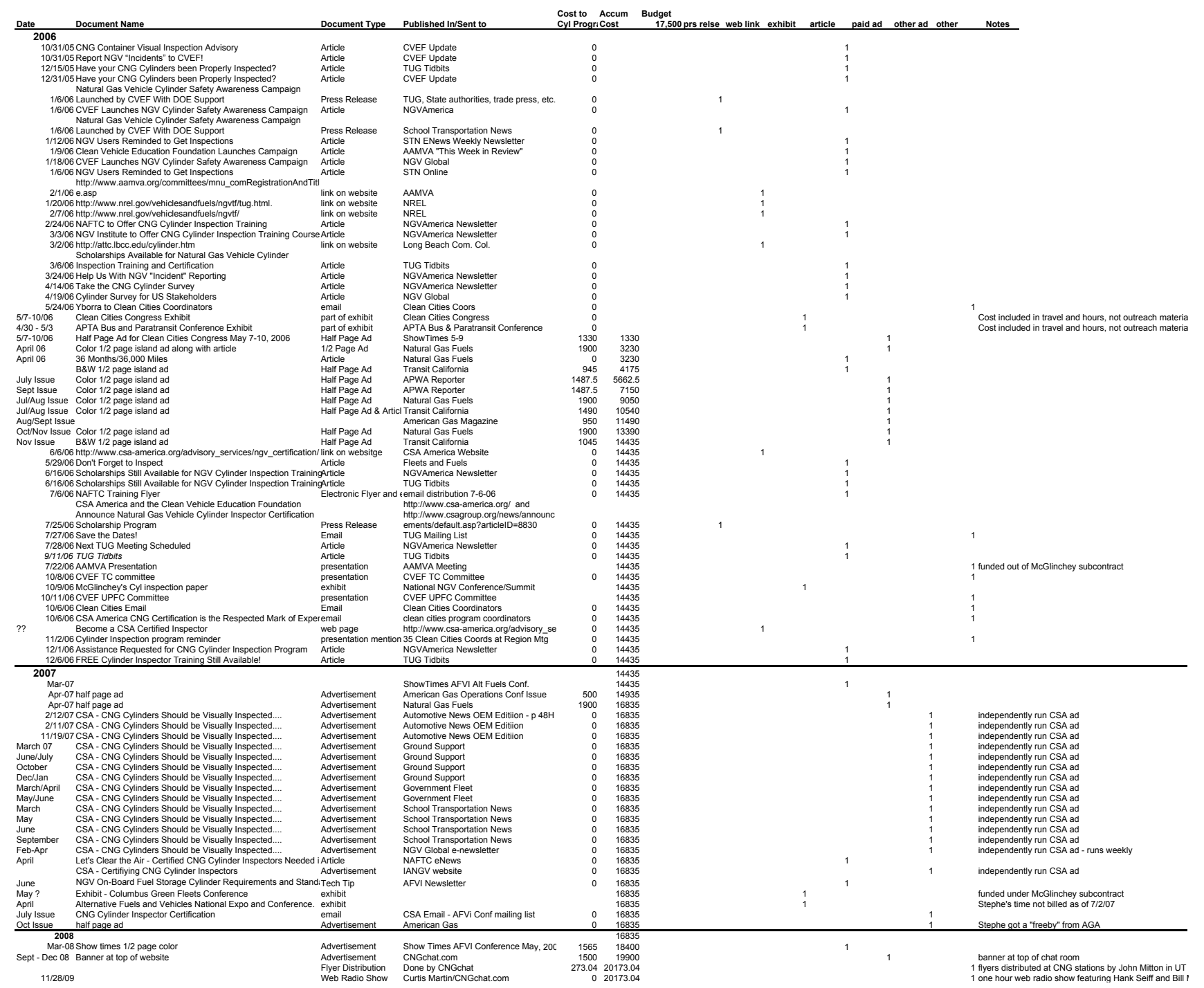



Appendix B - Suggested Wording for State PMVI (Periodic Motor Vehicle Inspection) and CVSA (Commercial Vehicle Safety Alliance) Commercial Vehicle Inspections (Last Revised 6-6-08)

\section{Compressed Natural Gas (CNG) Fuel Systems}

If the vehicle has a CNG fuel system which has not been disconnected and depressurized:

Examine the fuel system and reject vehicle for:

Any fuel leakage from the CNG fuel system detected by smell (CNG is odorized), sound or visible evidence (such as ice buildup at fuel system connections and fittings), and verified by a commercial leak detection fluid.

Examine the CNG fuel system and reject vehicle if:

1. The tank(s), tank mounting system and fuel system are not in serviceable condition and/or not securely attached or critical components are missing, disconnected, broken or loose.

2. There is obvious collision, chemical attack or fire damage to the fuel system. However the vehicle need not be rejected if it can be determined it has passed a detailed visual inspection (see below) since the time of the damage.

3. The vehicle system service (working) pressure on the label at the fueling connection receptacle is higher than the cylinder service (working) pressure on the cylinder label.

4. There is inadequate clearance to assure protection from mechanical damage or from the exhaust system.

Examine the CNG fuel cylinder(s) labels and reject vehicle if:

1. Information on cylinder manufacturer, service pressure, and "do not use after" date is missing or illegible

2. "Do not use after" date has passed

Reject vehicle if fuel cylinder(s) have not had a detailed visual inspection within the last three years or 36,000 miles, whichever is less. ${ }^{*}$ Inspection may be documented by inspection labels or tags on the cylinders, inspection labels on the windshield, doorpost, etc., and/or by other documentation which the vehicle owner/operator may provide.

Reject vehicle if there is obvious serious damage or deterioration to the CNG fuel system.

* refer to §2.1.3 ANSI NGV 2, FMVSS 304 or other applicable federal, state or local standard.

Safety Note: CNG is extremely flammable. Avoid exposure to any ignition source if leakage is suspected. CNG rises so beware that leaking CNG may collect in pockets on the ceiling of structures and form flammable mixtures. Do not bring leaking (or suspected leaking) vehicles indoors; park leaking CNG vehicles outside in an uncovered location. 


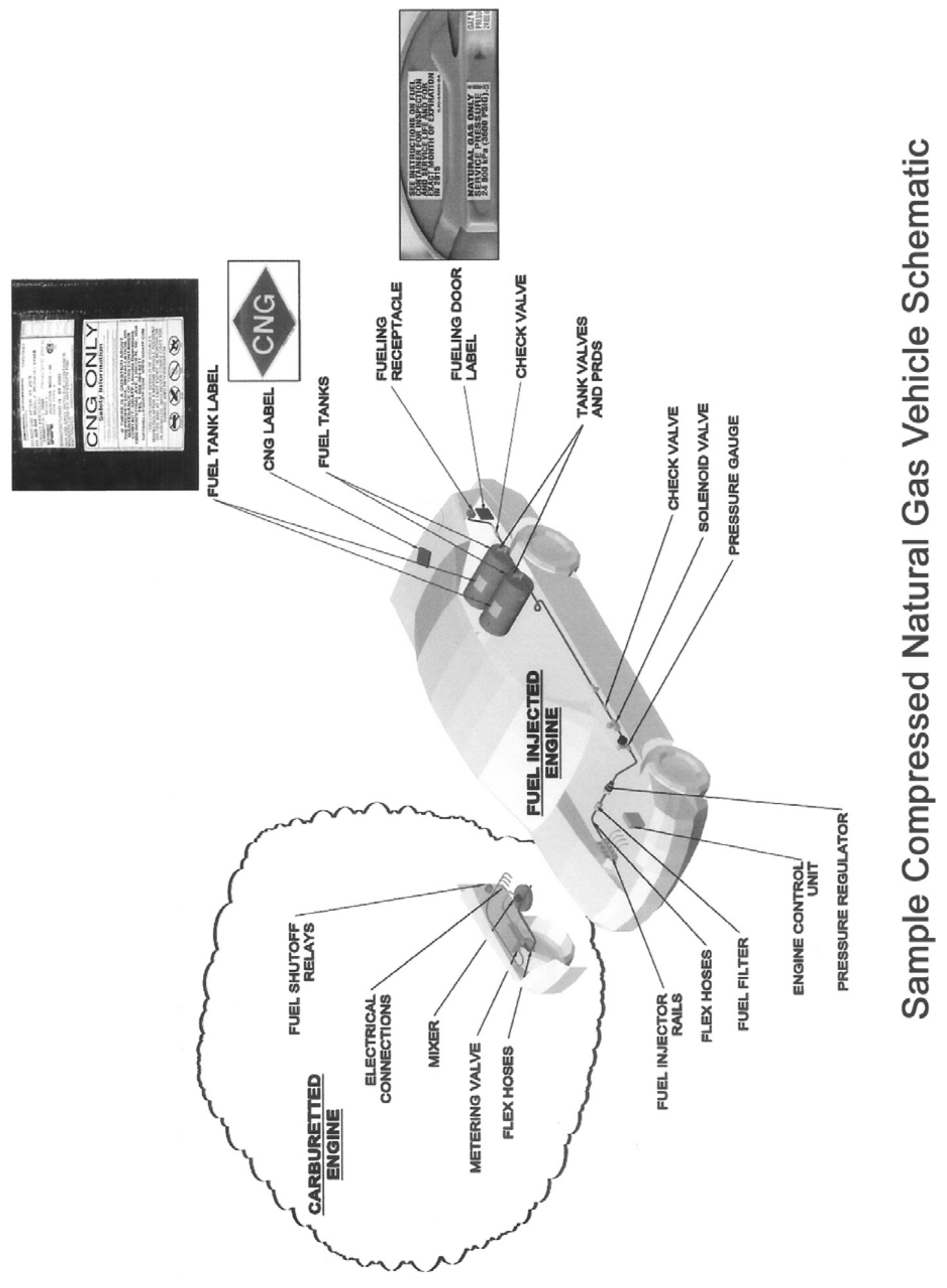


Appendix C - New NFPA 52 Dispenser Wording and Fueling Station Hand Out

8.14.12* A warning sign(s) with the words

"A. STOP MOTOR

B. NO SMOKING

C. FLAMMABLE GAS

D. NATURAL GAS VEHICLE FUEL CYLINDERS SHOULD BE PERIODICALLY INSPECTED (NORMALLY EVERY 3 YEARS) TO ENSURE SAFE OPERATION OF THE VEHICLE. CONTACT VEHICLE OR CYLINDER MANUFACTURER" shall be posted at the dispensing points. 


\section{How to Tell if Your Compressed Natural Gas (CNG) Fuel Cylinders Have Been Inspected}

The Department of Transportation requires this statement on the label of all CNG cylinders used on motor vehicles:

THIS CONTAINER SHOULD BE VISUALLY INSPECTED AFTER A MOTOR VEHICLE ACCIDENT OR FIRE AND AT LEAST EVERY 36 MONTHS OR 36,000 MILES, WHICHEVER COMES FIRST, FOR DAMAGE AND DETERIORATION

Evidence that your cylinders have been inspected could be:

1. a readily-visible inspection label on the cylinder:

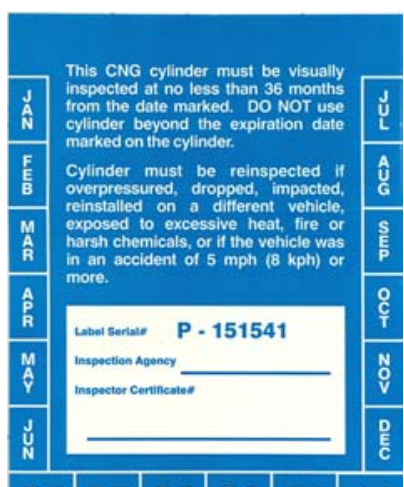

2. inspection form/report provided by inspector (perhaps kept in glove box with insurance, registration, etc. papers).

3. other - sticker on windshield, doorpost, fueling receptacle area, etc. 


\section{Appendix D - Summary of International Inspection and Recertification Activities}

Task: Determination of how other countries are handling the inspection and recertification of NGV cylinders.

This task was accomplished by surveying a number of colleagues/contacts in various countries where NGVs are active. A separate survey of users of NGVs in the United States on the need for an effort to permit recertification of cylinders beyond their initial 15-20 year life was conducted separately.

The following questions were posed to colleagues/contacts in Canada, Japan, Italy, Argentina, France, Brazil and Pakistan:

1. What is the lifetime of the CNG cylinder? Are there provisions to extend the lifetime of the cylinder?

2. What are the current requirements for cylinder recertification? For example, annual visual inspection to a maximum lifetime of 15 years, 5 year hydrostatic retest until the cylinder fails, etc.

3. What are the accepted inspection and recertification procedures? For example, visual, hydrostatic, ultrasonic, acoustic emission, etc. Is it a function of cylinder type?

4. How is the cylinder inspection/recertification enforced or implemented?

The following is a summary of the survey results:

\section{Canada}

Canada has a provision for the recertification of type 1 (all-steel) NGV cylinders in Supplement No. 1 to CSA B51-03, Part 2, "High Pressure Cylinders for the On-Board Storage of Natural Gas and Hydrogen as Fuels for Automotive Vehicles." The procedure is described in Annex B, "Procedure for Requalifying All-Steel Natural Gas Vehicle Storage Cylinders." The procedure requires a visual inspection in accordance with CGA C-6 followed by ultrasonic inspection per Annex B of ISO 9809-1. The cylinders are subsequently recoated and stamped. Steel vehicle cylinders can be requalified for at most 15 years from the date of ultrasonic inspection or 30 years from the date of manufacture, whichever comes first.

$\underline{\text { Japan }}$

In Japan, NGV cylinder lifetime is 15 years with no provisions to extend the life. A cylinder is first inspected after 4 years in service, followed by once every 25 months. The inspection methods employed include visual inspection and leak check. Damage is classified according to severity by cylinder type as specified by Japanese regulations. Leak checks are performed with natural gas and detector or soap solution. The inspection/recertification is enforced by a law which regulates high pressure gas safety. 
$\underline{\text { Italy }}$

NGV cylinders manufactured to the Italian regulation have a maximum lifetime of 40 years, whereas cylinders manufactured to the European regulation ECE R110 have a maximum lifetime of 20 years. The periodic retest for Italian regulation cylinders is conducted every 5 years and includes a hydraulic test at 300 bar, weight measurement and internal/external visual inspection. The periodic retest for R110 cylinders is every 4 years and includes a visual inspection for type 1 cylinders and a hydraulic test to 300 bar for types 2-4 cylinders. An Italian laboratory organization (SFBM) is responsible for the requalification of cylinders.

\section{$\underline{\text { Argentina }}$}

There is currently no defined lifetime for NGV cylinders in Argentina, with the exception of ECOTEMP (Russian) cylinders which were manufactured in accordance with NGV2 (15 years). Cylinders are recertified via visual inspection and hydrostatic retest every 5 years until the cylinder fails. The requalification requirement was established in 1991 and is regulated by the state gas authority (Gas del Estado).

\section{France}

CNG cylinders are requalified every 4 years in France in accordance with the ECE R110 regulation (20 year service life). A French group (Cetim) has developed an inspection system which eliminates the need for internal visual inspection and hydrostatic testing. The system includes a web-based database which keeps a written and visual history of each cylinder. The cylinders are inspected in-situ under full gas pressure using a number of nondestructive testing methods including visual, video-scope, and bubble leak test.

\section{$\underline{\text { Brazil }}$}

The lifetime of type $1 \mathrm{CNG}$ cylinders in Brazil is 20 years and there are currently no provisions to extend their life. Cylinder recertification occurs every 5 years and for the case of type 1 vessels includes thread inspection, external/internal visual inspection, hydrostatic test, weight measurement and inspection of labels. Types 2-4 are subjected to additional tests in accordance with ISO 11623.

\section{$\underline{\text { Pakistan }}$}

CNG cylinder lifetime is a function of the standard to which it was manufactured (e.g. NGV2 $=15-20$ years). Cylinders are currently requalified every 5 years via visual inspection and hydrostatic testing. Requalification is enforced at the refueling station through the verification window decals which specify cylinder retest dates. 
Appendix E - Review of Cylinder and CNG Fuel System Training Safety Requirements and Actual Practices

31 Fleets Responding on Cylinder Inspection and Safety Practices:

Capital City Coach, Indianapolis, IN

Carlos Garcia, Ontario, CA

Citizens Gas and Coke, Indianapolis, IN

City of Asheville, NC

City of Kirkwood, MO

City of Long Beach, CA

City of Merced, CA

City of Tulare, CA

City of Visalia, CA

Fiba Canning, Scarborough, Ontario, Canada

Greater Portland Transit, Portland, ME

Greenes Auto Service, Indianapolis, IN

Hoffman Beverage, Virginia Beach, VA

Lansing Community College, Lansing, MI

Manhattan Beer, New York City, NY

Monterey-Salinas Transit, Monterey, CA

Montgomery County, MD

MV Transit, Fremont, CA

Oklahoma Natural Gas, Davenport, OK

Omnitrans, San Bernardino, CA (2)

QCMetrolink, Rock Island, IL

Questar, Salt Lake City, UT

Sacramento Regional Transit District, Sacramento, CA

Santa Clarita Transit, Santa Clarita, CA

SunLine Transit, Thousand Palms, CA

SW Transit Agency, Caruthers, CA

Valley Transit, Walla Walla, WA

Veolia Transportation, Chula Vista, CA

Veolia Transportation, Springfield, VA

West Wind Farms, Deer Lodge, TN

14 Fleets Visited in Person or by Phone:

Alternate Fuel Systems, St. Louis, MO

Citizens Gas and Coke, Indianapolis, IN

GSA Fleets in Western Washington State, Ft. Lewis, WA

Laclede Gas, St. Louis, MO

Lower Merion School District, Ardmore, PA

Oklahoma Natural Gas, Davenport, OK

Omnitrans, San Bernardino, CA 
Pierce Transit, Tacoma, WA

Questar, Salt Lake City, UT

South Coast Area Transit (now Gold Coast Transit), Oxnard, CA

SeaTac Airport, Seattle, WA

Shuttle Express, Seattle, WA

Valley Transit, Walla Walla, WA

WE Energy, Milwaukee, WI 


\section{Natural Gas Vehicle CNG Cylinder Inspection Training and Certification Scholarship Program}

The Department of Transportation requires each vehicular CNG container (cylinder) to carry a label saying:

CSA is the only nationally recognized organization certifying CNG cylinder inspectors in the US and
THIS CONTAINER SHOULD BE VISUALLY INSPECTED AFTER A MOTOR VEHICLE ACCIDENT OR FIRE AND AT LEAST EVERY 36 MONTHS OR 36,000 MILES, WHICHEVER COMES FIRST, FOR DAMAGE AND DETERIORATION

Canada. The inspector training and testing program and a list of certified inspectors is available at: http://webext.csa.ca/cng/cngmain.asp\#searchinspector. The organizations listed below provide training for and administer the CSA certification test:

National Alternative Fuels Training Consortium - http://www.naftc.wvu.edu/ Contact: Al Ebron (al.ebron@mail.wvu.edu or 304-293-7882)

AFV International - www.afvtraining.net Contact: Bill McGlinchey (w.mcglinchey@worldnet.att.net or 304-296-6568)

Natural Gas Vehicle Institute - http://www.ngvi.com/ Contact: Leo Thomason (leo@ngvi.com or 800-510-6484)

Advanced Transportation Technology - http://www.attcolleges.org/att coleges.html Contact: Cal Macy (cmacy@lbcc.edu or 562-938-3067)

Energy Transfer Technology - http://www.energytransfertechnology.com/auto truck \& fleet.htm Contact: Scott Hammer (trainingresults@verizon.net or 814-455-4024)

The US Department of Energy, through the Clean Vehicle Education Foundation (CVEF), offers training and certification testing scholarships for qualifying technicians or organizations. These scholarships will reimburse you for successfully completing a cylinder inspector training program provided by one of these organizations and passing the CSA test.

To apply for a scholarship:

1) Contact one of the organizations listed above and determine when and where you want to take their course. You may take a course at their facility or work with them to provide a course for a number of technicians at a facility of your choice.

2) Fill out the form and return it to Hank Seiff, Director of Technology, Clean Vehicle Education Foundation, 6812 Haycock Road, Falls Church, VA 22043, FAX: 703-534-6151.

3) We will determine whether scholarship money is available for you, based on the information included in your request and availability of program funding (at this time funding is available only through the end of 2008). We will inform you of our decision within 10 business days of receiving your application. If we agree to pay for your training and testing, you must make your own arrangements with the training and testing facility and provide us with a receipt for the amount paid and documentation that you successfully completed the course and passed the test. You are responsible for your own travel, lodging and expenses (other than training tuition and testing cost), unless special plans are made in advance.

4) Questions? - Call or email Hank Seiff, Director of Technology, Clean Vehicle Education Foundation, 703-534-6151, hseiff@cleanvehicle.org. 


\section{CNG Cylinder Inspection Training and Certification Scholarship Form}

Name:

Title:

Company:

Address:

Phone/FAX:

Email Address:

From what organization do you plan to take training?

When and where will the training course be held?

If you plan to have the course administered to a group or away from the trainer's facility, tell us the details:

What is the cost of this course? (If you plan to provide the course to more than one person, provide details of total cost and number of people taking the course)

When/where/for whom do you plan to inspect CNG cylinders? How will your training and certification be useful in assuring the safety of compressed natural gas vehicles?

Why do you feel scholarship money should be provided for you or your group to be trained and certified?

Is there a special need for scholarship money to pay for travel, lodging and other expenses? If so, tell us what that special need is and itemize the extra amount you would like us to provide.

Signature:

Date: 


\section{Appendix G - Sample Correspondence with Applicant}

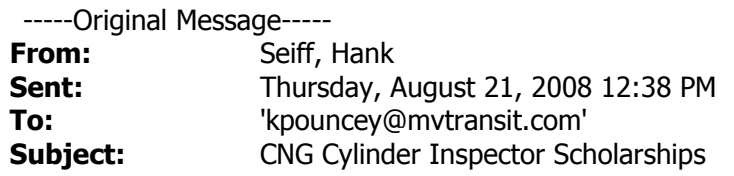

Mr. Pouncy:

On May 1 we approved your scholarship request and agreed to pay for you to take the CNG Cylinder Inspector Certification course and test. As yet we haven't received the documentation of your successful completion of the course and the test.

In order to reimburse you for your costs, we need to receive documentation that you successfully passed the course and test and the amounts paid for them. Please forward this information via email to hseiff@cleanvehicle.org, fax to 703-534-6151 or mail to me at CVEF, 6812 Haycock Road, Falls Church, VA 22043.

We're looking forward to hearing from you.

Hank Seiff

Director of Technology

Clean Vehicle Education Foundation

$\begin{array}{ll}\text { From: } & \text { Seiff, Hank } \\ \text { Sent: } & \text { Thursday, May 01, 2008 2:10 PM } \\ \text { To: } & \text { 'Kenneth Pouncey' } \\ \text { Subject: } & \text { RE: Scholarship Response }\end{array}$

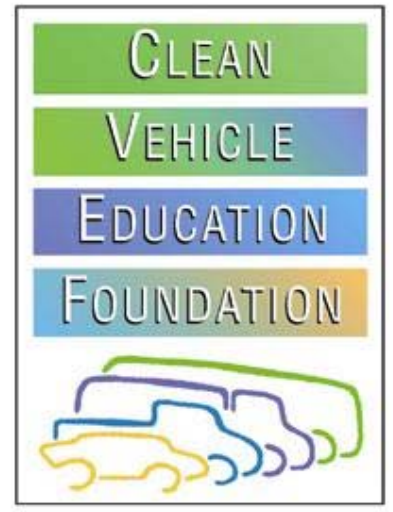

Mr. Kenny Pouncey

Regional Director of Maintenance

MV Transportation

360 Campus Lane, Suite 201
Clean Vehicle Education Foundation

6812 Haycock Road

Falls Church, VA 22043

May 1, 2008 
Fairfield, CA 93454

Dear Mr. Pouncy:

We have received and approve your request for scholarship funding for CNG cylinder inspection training and certification. We agree to pay up to $\$ 495$ for the course tuition and $\$ 150$ for testing costs, provided you meet the requirements of the next paragraph.

It is your responsibility to make arrangements with the training and testing facility and provide us with receipts for the amount paid, and documentation that you successfully completed the course and passed the CSA test. We will then reimburse you for training and testing costs. You or your employer are responsible for any other costs.

We are happy to assist in training and certifying CNG cylinder inspectors. Natural gas vehicles decrease harmful emissions and reduce America's dependence on foreign oil. Your skills will help assure the continued safety of the natural gas vehicle fleet.

Please contact me (703-534-6151, hseiff@cleanvehicle.org) if you have any questions.

Sincerely,
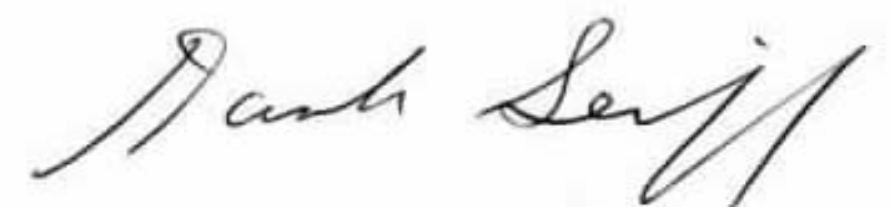

Hank Seiff

Director of Technology

Clean Vehicle Education Foundation

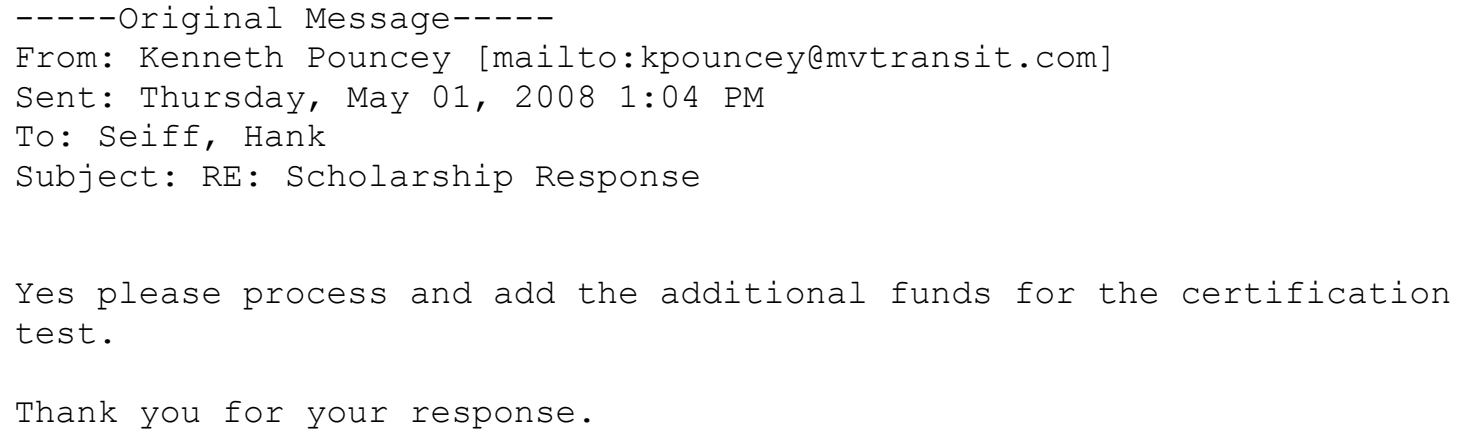




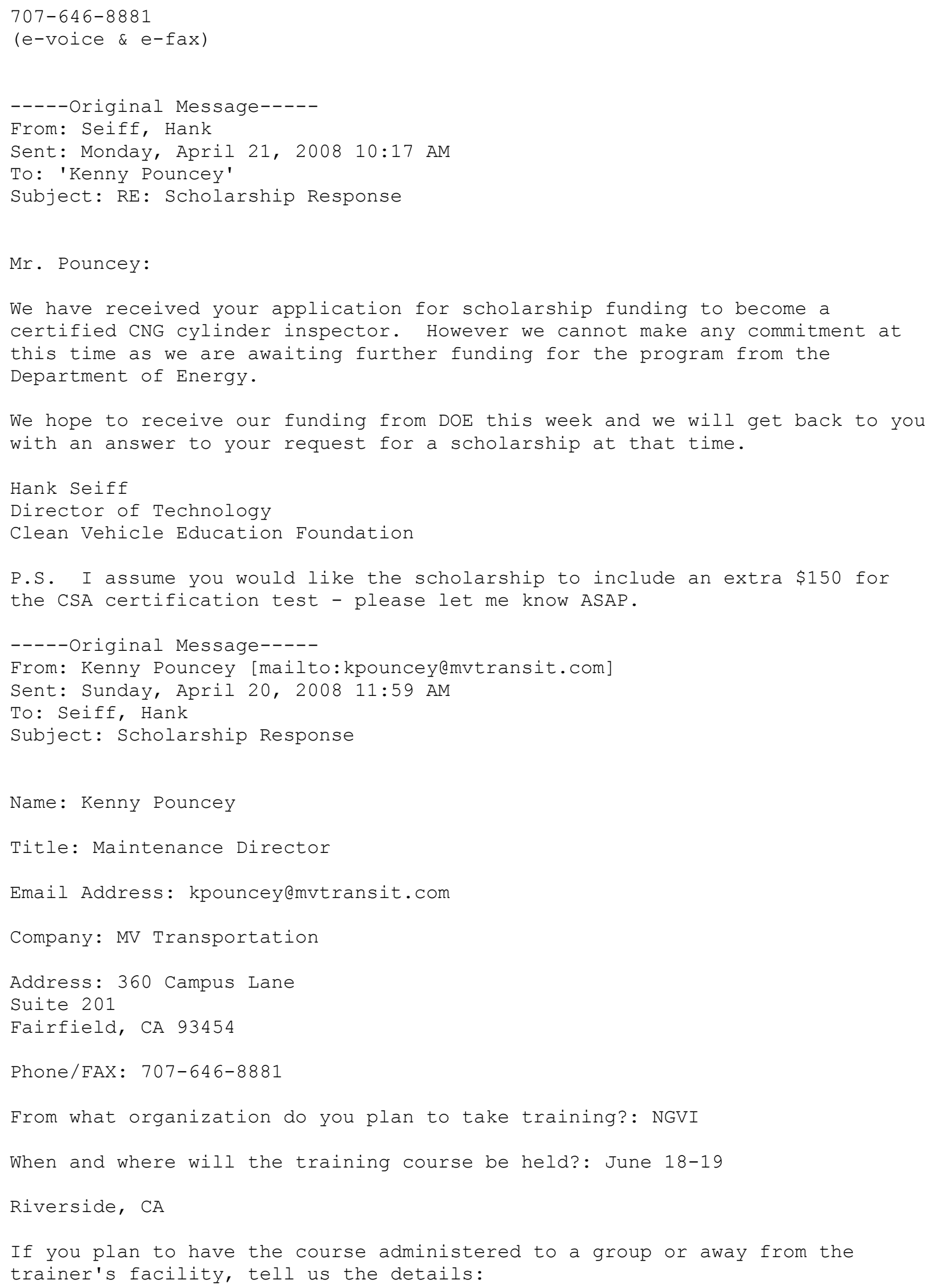


What is the cost of this course? (If you plan to provide the course to more than one person, provide details of total cost and number of people taking the course): $\$ 495$

When/where/for whom do you plan to inspect CNG cylinders? How will your training and certification be useful in assuring the safety of compressed natural gas vehicles?: We have mulitple fleets in the greater LA area that utilize CNG. Will be the designated person to keep inspections current

Why do you feel scholarship money should be provided for you or your group to be trained and certified?: We are a contractor to multiple goverment agencies and funding is limited.

Is there a special need for scholarship money to pay for travel, lodging and other expenses? If so, tell us what that special need is and itemize the extra amount you would like us to provide: No program is close to my home. 
Appendix H - Scholarship Record
CNG Cylinder Inspection Training and Certification Scholarships

\begin{tabular}{|c|c|c|c|c|c|c|c|c|c|c|c|c|c|c|c|c|c|c|c|}
\hline \multirow{2}{*}{\multicolumn{2}{|c|}{ №. Person }} & \multirow[b]{2}{*}{ Tithe, Company, Address } & & & & & & & & & \multirow{2}{*}{\multicolumn{3}{|c|}{ 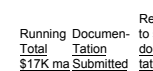 }} & & \\
\hline & & & Phone, FAX, Email & $\frac{\frac{\text { Form Sub- }}{\text { mited on }}}{\frac{A p}{D^{2}}}$ & 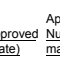 & $\begin{array}{l}\text { ed } \\
0\end{array}$ & $\begin{array}{l}\text { Approvec } \\
\text { Iolat }\end{array}$ & 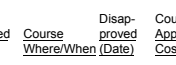 & 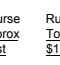 & 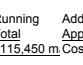 & & & & $\begin{array}{l}\text { minder } \\
\text { unenen } \\
\text { one }\end{array}$ & $\begin{array}{l}\text { wal } \\
\text { st }\end{array}$ & $\begin{array}{l}\text { Sobmtg } \\
\text { or Pymt }\end{array}$ & $\begin{array}{l}\text { a aid } \\
\text { Date) }\end{array}$ & 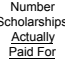 & ss \\
\hline & 1 Kenneth Conaway & 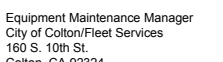 & 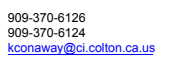 & 1/31/106 & 2/2/106 & 1 & 1 & $\underset{4 / 19-20}{1 \mathrm{NGVI}}$ & $\$ 545$ & $\$ 545$ & & so & & 810 & & & & & \\
\hline & 2 Jose Esparza & 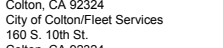 & $\begin{array}{l}909-370.6126 \\
909-370-6124\end{array}$ & 1/31/106 & 212/106 & 1 & 1 & $\underset{4 / 19-20}{2 \mathrm{NGV}}$ & $\$ 545$ & $\$ 1,990$ & & so & & 8110 & & & & & \\
\hline & 3 John Kanyan & 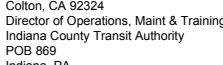 & 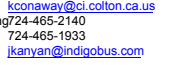 & 211106 & 212106 & 1 & & $\begin{array}{l}3 \mathrm{NGVI} \\
4 / 19-20\end{array}$ & $\$ 545$ & $\$ 1,635$ & & so & $6 / 1106$ & & $\$ 545.00$ & 6151506 & 661606 & 1 & \\
\hline & 4 John Duncan & 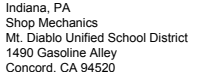 & 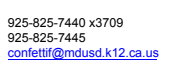 & 27706 & 27706 & 1 & 1 & $\begin{array}{l}4{ }_{4}^{4 N V V I} \\
4 / 19 \cdot 20\end{array}$ & $\$ 545$ & $\$ 2,180$ & 150.00 & $\$ 150$ & 6/12006 & & $\$ 6955.00$ & 6112066 & 6/19106 & 1 & \\
\hline & 5 Larry Hammil & 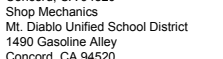 & 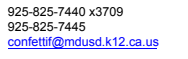 & 277106 & 271106 & 1 & 1 & $\begin{array}{l}5 \mathrm{NNVVI} \\
4 / 1 / 9 \cdot 20\end{array}$ & $\$ 545$ & $\$ 2,25$ & 150.00 & $\$ 300$ & 6/12/106 & & $\$ 695.00$ & 6/12/106 & 6/19/106 & 1 & \\
\hline & 6 Bryan Muramoto & $\begin{array}{l}\text { Maintenance Manager } \\
\text { MV Trasspotation, Inc. } \\
600 \text { Sutro St. }\end{array}$ & 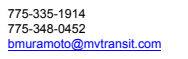 & 277106 & 277106 & 1 & & $\begin{array}{l}6 \mathrm{~N} \times \mathrm{VV} 1 \\
4 / 18-20\end{array}$ & $\$ 545$ & $\$ 3,270$ & & $\$ 300$ & 6/2/206 & & $\$ 545.00$ & $6 / 5106$ & $61 / 106$ & 1 & \\
\hline & 7 Les Fy & 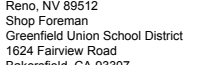 & 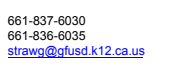 & 277106 & 277106 & 1 & 1 & $\begin{array}{l}7 \mathrm{NGVI} \\
4 / 19-20\end{array}$ & $\$ 545$ & $\$ 3,815$ & 125.00 & $\$ 425$ & & $8 / 10$ & & & & & \\
\hline & 8 Rick Statanaker & 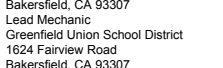 & 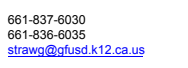 & 277106 & 277106 & 1 & 1 & $\begin{array}{l}8 \mathrm{NGVI} \\
4 / 19-20\end{array}$ & $\$ 545$ & $\$ 4,360$ & 125.00 & $\$ 550$ & & $8 / 10$ & & & & & \\
\hline & 9 Aaron Carson & 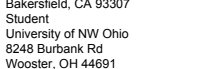 & 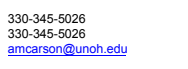 & 281806 & 29106 & 1 & & 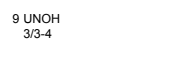 & $\$ 375$ & $\$ 4,735$ & & $\$ 550$ & 6/1/106 & & $\$ 375.00$ & $6 / 1 / 106$ & 61606 & 1 & \\
\hline & 10 Chris Czerwinski & 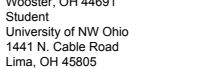 & 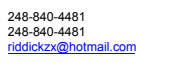 & 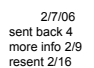 & 2117706 & 1 & & $\begin{array}{l}10 \mathrm{uNOH} \\
3 / 3-4\end{array}$ & $\$ 375$ & $\$ 5,110$ & & $\$ 550$ & & $8 / 10$ & & & & & \\
\hline & 11 Eric Jensen & 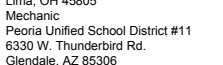 & $\begin{array}{l}\text { 623-486-6152 } \\
\text { 623-486-6152 } \\
\text { clo dgass@peoriaud.K12.az.us }\end{array}$ & 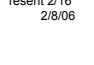 & 219106 & 1 & & $\underset{4 / 19-20}{11 \mathrm{NGV}}$ & $\$ 545$ & 55.655 & 200.00 & $\$ 750$ & $6 / 1406$ & & $\$ 745.00$ & 6/14/06 & 6/19/106 & 1 & \\
\hline & 12 David Ezzinga & 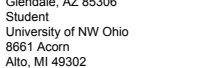 & 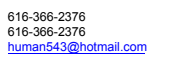 & 277106 & 219106 & 1 & & $12 \mathrm{UNOH}$ & $\$ 375$ & s6,030 & & $\$ 750$ & & $8 / 10$ & & & & 1 & \\
\hline & 13 Willam Clough & 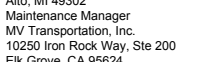 & 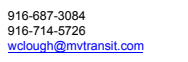 & 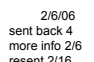 & & & & $12 \mathrm{NGVI}$ & & 56,030 & & $\$ 750$ & & & & & & & \\
\hline & 14 Will Wigal & 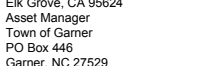 & 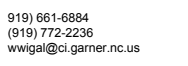 & 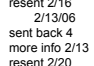 & & & & $12 \mathrm{NGVI}$ & & $\$ 6,030$ & & $\$ 750$ & & & & & & & \\
\hline & 15 Alan Vincent & 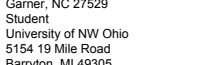 & 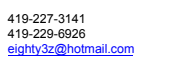 & $\begin{array}{l}\text { resestet2200 } \\
2 / 131006\end{array}$ & 2144106 & 1 & & 13 UNOH & $\$ 375$ & 56,005 & & $\$ 750$ & 811606 & $8 / 10 \mathrm{~s}$ & $\$ 375.00$ & $8 / 21106$ & $9 / 506$ & 1 & \\
\hline & 16 Joel Agner & 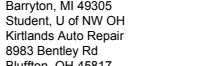 & $\begin{array}{l}\text { 419-35-4975 } \\
\text { jeel agnererenhoo.com }\end{array}$ & 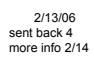 & 2144106 & 1 & & $\begin{array}{l}14 \mathrm{UNOH} \\
21177-18\end{array}$ & $\$ 375$ & $\$ 6,780$ & & $\$ 750$ & 6/1806 & & $\$ 375.00$ & 6/30/06 & 71506 & 1 & \\
\hline & 17 Hans White & 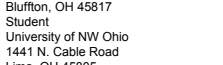 & $\begin{array}{l}\text { 419-998-3160 } \\
\text { hawhiteQunohe.edu }\end{array}$ & 2/1406 & 2144106 & 1 & & $\begin{array}{l}15 \mathrm{UNOH} \\
3 / 3.4 \\
3\end{array}$ & $\$ 375$ & $\$ 7,155$ & & $\$ 750$ & & $8 / 10$ & & & & & \\
\hline & 18 Jeff Krepshaw & 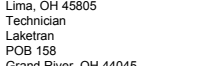 & 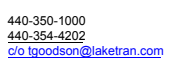 & $\begin{array}{l}\text { 21/406 } \\
\text { callod of more } \\
\text { info } 2 / 144\end{array}$ & 2146106 & 1 & & 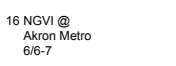 & $\$ 645$ & $\$ 7,800$ & & $\$ 750$ & & 9/19 & & & & & $\begin{array}{l}\text { John Avena (sp?) of Laketran called } 8 / 8 \text { - Itold him to get in touch with Sue White } \\
\text { of Akron RTA Who Id d just corresponded with. }\end{array}$ \\
\hline & 19 Dennis Wargo & 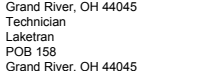 & 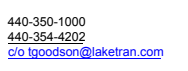 & 211506 & 2146/106 & 1 & & 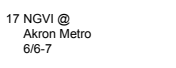 & $\$ 645$ & s8,445 & & $\$ 750$ & 9/2206 & $9 / 19 \quad \&$ & $\$ 645.00$ & 9/25106 & 10/4/06 & 1 & \\
\hline & 20 Mathew Lake & 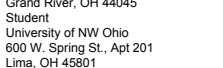 & 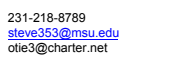 & 2/1406 & 2/14/06 & 1 & & $\begin{array}{c}18 \mathrm{UNOH} \\
\text { s/3.4- }\end{array}$ & $\$ 375$ & $\$ 8,820$ & & $\$ 750$ & & $8 / 10$ & & & & & \\
\hline & 21 Stephen Hull & 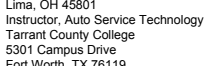 & 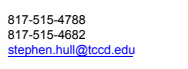 & 2/14406 & 2114/06 & 1 & & $\underset{3 / 13-14}{19 w / U}$ & $\$ 321$ & $\$ 9,141$ & 950.00 & $\$ 1,700$ & 7/177106 & & $\$ 1,271.00$ & 7/24106 & 7/24/06 & 1 & $\begin{array}{l}\text { agreed to pay high addl costs since he'd become an instructor of the course at his } \\
\text { home cologe }\end{array}$ \\
\hline & 22 Jeffrey Parks & 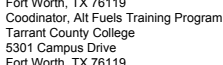 & 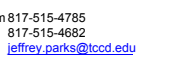 & 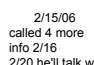 & & & & $\underset{3 / 13-14}{19 w v U}$ & & s9,141 & & $\$ 1,700$ & & & & & & & \\
\hline 23-37 & Cily of Anaheim & 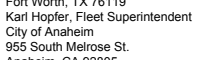 & $\begin{array}{l}\text { 714-765-6890 } \\
714-465-68999 \\
\text { khooperer@anaheim.net }\end{array}$ & $\begin{array}{l}2 / 20 \text { eilitak w } \\
2 / 15 / 100\end{array}$ & 21461/106 & 0 & & 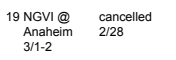 & & s9,141 & & $\$ 1,700$ & & & & & & & for 15 students \\
\hline & 38 Ted Rapley & 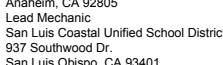 & 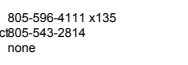 & 2/17/106 & 2117706 & 1 & & $\begin{array}{ll}20 \mathrm{NGVI} \\
4 / 19-20\end{array}$ & $\$ 545$ & $\$ 9,686$ & 186.50 & $\$ 1,887$ & $5 / 2206$ & & $\$ 731.50$ & $5 / 22106$ & 61606 & 1 & \\
\hline & 39 Brett Lavoy & 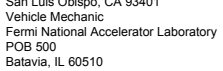 & $\begin{array}{l}\begin{array}{l}630-840-3307 \\
630-848-0530 \\
\text { clo davidson@ @nal.gov }\end{array}\end{array}$ & $\begin{array}{l}2117106 \\
\text { emaled } \\
\text { emal17106 }\end{array}$ & 2/20106 & 1 & & $21 \mathrm{NGVI}$ & $\$ 545$ & $\$ 10,231$ & 166.00 & $\$ 2,053$ & $6 / 506$ & & $\$ 711.00$ & $6 / 5106$ & 6/16/106 & 1 & \\
\hline
\end{tabular}




\begin{tabular}{|c|c|c|c|c|c|c|c|c|c|c|c|c|c|c|c|c|c|c|}
\hline 40 Joel Smith & $\begin{array}{l}\text { Quality Manager } \\
\text { FAB ndustries } \\
1417 \mathrm{~A} \text { Commerce Bivd }\end{array}$ & $\begin{array}{l}356-831-155 \\
25-631-7150 \\
\text { ismith frabind.com }\end{array}$ & 2/16/106 2 & 2/20106 & 1 & & ${ }_{3 / 13-14}^{22 w v}$ & $\$ 550$ & $\$ 10,781$ & & $\$ 2,053$ & $5 / 31 / 06$ & & $\$ 550.00$ & $6 / 1 / 106$ & $6 / 6 / 106$ & 1 & \\
\hline 41-44 Scott Bohannon & 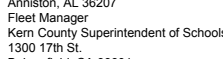 & $\begin{array}{l}\text { 661-852-5812 } \\
\text { Isscobohnon@kern.org }\end{array}$ & $\begin{array}{l}2 \text { 2/21/06 } \\
\text { emaled } \\
\text { more info } 2 / 21\end{array}$ & 2/2206 & 1 & 1 & $\underset{23 \mathrm{NGV}}{23 / 19-20}$ & $\$ 1,980$ & $\$ 12,761$ & & $\$ 2,053$ & 2212307 & 8110 & $\$ 1,485.00$ & $2 / 266707$ & $2 / 26107$ & 3 & only 3 passed first time around \\
\hline 45 Albert Rodriguez & 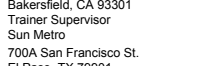 & 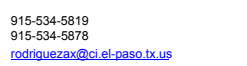 & $\begin{array}{l}\text { 2012106 } \\
\text { emiled proposal } \\
2,21 \text { r resend } 37\end{array}$ & & & & $\begin{array}{ll}23 \mathrm{NGVY} \\
4 / 1 / 9-20\end{array}$ & & $\$ 12,761$ & & $\$ 2,053$ & & & & & & & \\
\hline 46-47 Sam Amentrout & 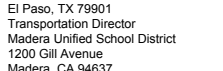 & $\begin{array}{l}699.673-2288 \\
559-675-58545 \\
\text { ammentrout s@madera.k12.ca.us }\end{array}$ & 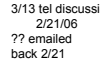 & sion $3 / 206$ & 2 & 2 & $\begin{array}{l}25 \mathrm{NGVI} \\
\text { date unknown }\end{array}$ & $\$ 1,090$ & $\$ 13,851$ & & $\$ 2,053$ & & $8 / 10$ & & & & & \\
\hline 48 Michael Gedeon & 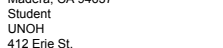 & 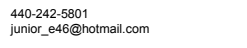 & $2 / 22=$ & 2/27106 & 1 & & ${ }_{3 / 3-4}^{26} \mathrm{UNOH}$ & $\$ 375$ & $\$ 14,226$ & & $\$ 2,053$ & 9/20/06 & $8 / 10$ & $\$ 375.00$ & $9 / 21 / 06$ & 10/4/106 & 1 & $\begin{array}{l}\text { sent CSA certificate but nothing else, wrotel bock and forth in June. } \\
\text { Final bit of info submitted } 9 / 20\end{array}$ \\
\hline 49 Darren Sell & 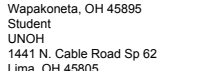 & 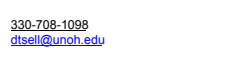 & 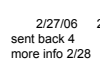 & $2 / 28106$ & 1 & & ${ }_{27 / 3-4}^{27}$ & $\$ 375$ & $\$ 14,601$ & & $\$ 2,053$ & & $8 / 10$ & & & & & \\
\hline 50-61 Joe LaFreniere & 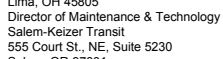 & 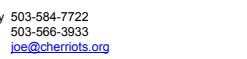 & 2/23/06 & 3/2/106 & 12 & & $\begin{array}{l}39 \text { Portland } \\
\text { Conmunty } \\
\text { Col } 4 / 111-12\end{array}$ & $\$ 4,620$ & $\$ 19,221$ & & $\$ 2,053$ & $\begin{array}{l}67106 \\
977106 \\
-10\end{array}$ & & $\begin{array}{l}\$ 3.465 .00 \\
\$ 877.00\end{array}$ & $\begin{array}{r}6 / 12106 \\
97 / 106\end{array}$ & $\begin{array}{r}6 / 16 / 106 \\
9 / 8106\end{array}$ & $\frac{9}{2}$ & 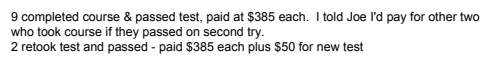 \\
\hline 62 Jerry Singh & 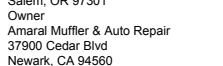 & 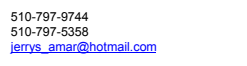 & 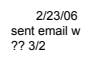 & 3/6/106 & 1 & 1 & $\begin{array}{l}40 \text { NoVI } \\
4 / 18-20\end{array}$ & $\$ 545$ & $\$ 19,766$ & & $\$ 2,053$ & & $8 / 10$ & & & & & \\
\hline 63 Art Prendergast & 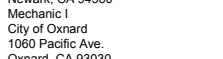 & $\begin{array}{l}\text { 805-385-8048 } \\
\text { 805-255-8053 } \\
\text { ken.dunham@oci.oxnard.ca.us }\end{array}$ & 21/2206 & & 0 & & 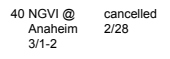 & & $\$ 19,766$ & & $\$ 2,053$ & & & & & & & \\
\hline 64 Andrew Sandstrom & 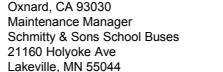 & 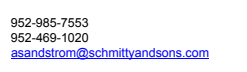 & 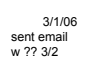 & $3 / 6106$ & 1 & & $\begin{array}{l}41 \text { NGVI } \\
4 / 19.20 \text { or } \\
11131-1211106\end{array}$ & $\$ 545$ & $\$ 20,311$ & $\$ 215.00$ & $\$ 2,268$ & 6/15106 & & $\$ 760.00$ & $6 / 15106$ & 6/19/06 & 1 & \\
\hline 65-84 Patrick Foley & 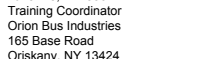 & $\begin{array}{l}315-233.5250 \\
315-768-52020 \\
\text { pfoley@eorionus.com }\end{array}$ & 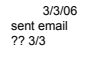 & & 0 & & $\begin{array}{c}41 \text { NGI } \\
\text { Oriskany } \\
\text { no date }\end{array}$ & & $\$ 20,311$ & & $\$ 2,268$ & & & & & & & \\
\hline 85-86 Mike Klinkner & 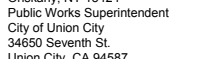 & $\begin{array}{l}510-675-5371 \\
510-6579349 \\
\text { mklinker@ociunion-cily.ca.us }\end{array}$ & $\begin{array}{l}\substack{2 / 17106 \\
\text { sentemail } \\
? 23 / 6} \\
3 / 6\end{array}$ & $3 / 7106$ & 2 & 2 & 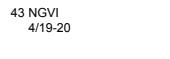 & $\$ 1,090$ & $\$ 21,401$ & $\$ 345.00$ & $\$ 2,613$ & & 8110 & & & & & \\
\hline 87-88 Danny Akers & 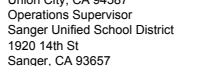 & 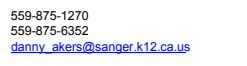 & $\begin{array}{l}316106 \\
\text { CANCELLED }\end{array}$ & $3 / 6106$ & & & $\begin{array}{ll}43 \mathrm{NGV} \\
4 / 19-20\end{array}$ & & $\$ 21,401$ & & $\$ 2,613$ & & & & & & & \\
\hline 89-97 Roger Wilson & 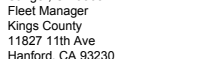 & 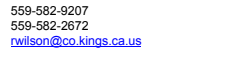 & $\begin{array}{l}3 / 16106 \\
\text { sent } \\
? 2316\end{array}$ & 377106 & 9 & 9 & 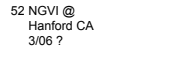 & $\$ 5,950$ & $\$ 27,351$ & & $\$ 2,613$ & & $8 / 10$ & & & & & \\
\hline 98 Art Prendergast 2 & 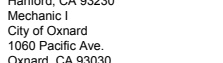 & 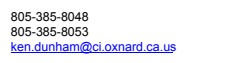 & 381806 & 3/9106 & 1 & 1 & $\begin{array}{ll}53 \mathrm{LBCC} \\
3 / 30-31\end{array}$ & $\$ 399$ & $\$ 27,750$ & & $\$ 2,613$ & & $8 / 10$ & & & & & \\
\hline 99-100 Terry Holden & 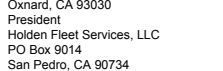 & 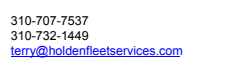 & 391066 & 3/13106 & 2 & 2 & $\begin{array}{ll}55 \mathrm{LBCC} \\
3 / 30-31\end{array}$ & $\$ 798$ & $\$ 28,548$ & & $\$ 2,613$ & $6 / 22106$ & & $\$ 798.00$ & 6/292/106 & 715106 & 2 & \\
\hline 101-111 Steve Smith & $\begin{array}{l}\text { Aminisistrive Analyst } \\
\text { City for nglewood }\end{array}$ & 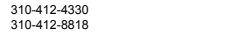 & $\begin{array}{ll}3 / 15 / 106 & 3 \\
\text { sent mail } ? 2 \mathrm{e}^{3}\end{array}$ & $\begin{array}{l}3 / 24106 \\
33116\end{array}$ & 11 & 11 & $\begin{array}{l}66 \text { LECC 3300-31 } \\
\text { \& 2nd date TBD }\end{array}$ & $\$ 4,389$ & $\$ 32,937$ & & $\$ 2,613$ & & 8110 & & & & & Revised application coming \\
\hline 112 Herman Cevantes & 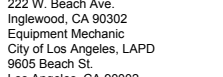 & 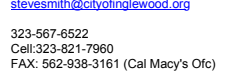 & $\begin{aligned} 3 / 683 / 23 \text { ressin } \\
3 / 23\end{aligned}$ & sponse & 1 & 1 & $\begin{array}{c}67 \mathrm{LCCC} \\
3 / 30.31\end{array}$ & $\$ 399$ & $\$ 33,336$ & & $\$ 2,613$ & & 8110 & & & & & \\
\hline 113 Mahendra Chahal & 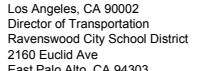 & 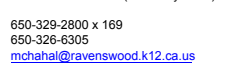 & $\begin{array}{l}\text { 3/23 } \\
\text { sent emaip prope } \\
3 / 27 \text { response }\end{array}$ & 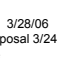 & 1 & 1 & $\begin{array}{lll}68 \mathrm{NVVV} \\
4 / 1 / 9-20\end{array}$ & $\$ 545$ & $\$ 33,881$ & $\$ 179.70$ & $\$ 2,792$ & & $8 / 22$ & & & & & \\
\hline 114 Jon Merry & 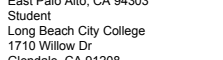 & $\begin{array}{l}\text { 818-247-2021 } \\
\text { ciontre@earthink.net }\end{array}$ & $3 / 27$ & 3/28106 & 1 & 1 & $\begin{array}{l}69 \mathrm{LBCC} \\
3 / 30-31\end{array}$ & $\$ 399$ & $\$ 34,280$ & & $\$ 2,792$ & 9/25/106 & $8 / 22$ & $\$ 399.00$ & 9/28/106 & 10/4/106 & 1 & \\
\hline $\begin{array}{l}\text { 115-116 Danny Akers } \\
\text { Don Lentz }\end{array}$ & 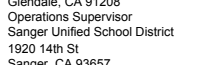 & 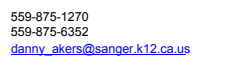 & $3 / 30$ & 4/406 & 2 & 2 & 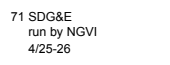 & so & $\$ 34,280$ & $\$ 570.00$ & $\$ 3,362$ & 7/20066 & & $\$ 570.00$ & 7/24/106 & $7 / 24 / 06$ & 2 & \\
\hline 117 Martin Vargas & 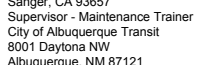 & 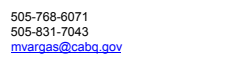 & 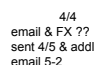 & & & & 71 & & $\$ 34,280$ & & $\$ 3,362$ & & & & & & & \\
\hline 118 Earl Green & 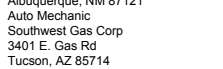 & 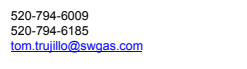 & 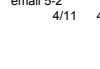 & 4/121206 & 1 & & $\begin{aligned} 72 \mathrm{NGV} \\
\text { revonix } \\
5 / 111-12\end{aligned}$ & $\$ 545$ & $\$ 34,825$ & & $\$ 3,362$ & $7 / 6106$ & & $\$ 545.00$ & 7/10/06 & 7/11/106 & 1 & \\
\hline 119 Matthew Campbell & 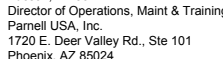 & $\begin{array}{l}\text { ng623.-581-8335 } \\
\text { meceparnellusa.com }\end{array}$ & 4144 & 4/14106 & 2 & & $\begin{array}{l}74 \mathrm{NGV} \\
\text { Phonix } \\
5 / 11-12\end{array}$ & $\$ 1,090$ & $\$ 33,915$ & & $\$ 3,362$ & & $8 / 22$ & & & & & \\
\hline 120-134 Matt Bean & 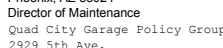 & 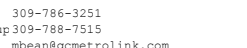 & ${ }_{\text {email ? ? }}^{4 / 14}$ & & & & 74 & & $\$ 35,915$ & & $\$ 3,362$ & & & & & & & Later emails indicate he's going to send people to TUG meeting in LA for training \\
\hline 135 David Cleme & 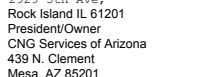 & $\begin{array}{l}488-4661-5166 \\
480-615-4338 \\
\text { dave@engaz.com }\end{array}$ & 4/14406 & 4/17706 & 1 & & $\begin{array}{c}75 \mathrm{NGV} \\
\text { Phonix } \\
5 / 11-12\end{array}$ & $\$ 545$ & $\$ 36,460$ & & $\$ 3,362$ & 7/171/106 & & $\$ 545.00$ & $7 / 24 / 06$ & $7 / 24 / 06$ & 1 & \\
\hline 136-139 Will Chrisman & 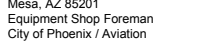 & $\begin{array}{l}\text { 602-273-2007 } \\
\text { wilard.chrisman@phoenix.gov }\end{array}$ & 4/24106 & 4/24/106 & 4 & & $\begin{array}{ll}79 \text { NGVI } \\
\text { Phoenix }\end{array}$ & $\$ 2,180$ & 8,640 & & $\$ 3,362$ & & $8 / 2$ & & & & & \\
\hline
\end{tabular}




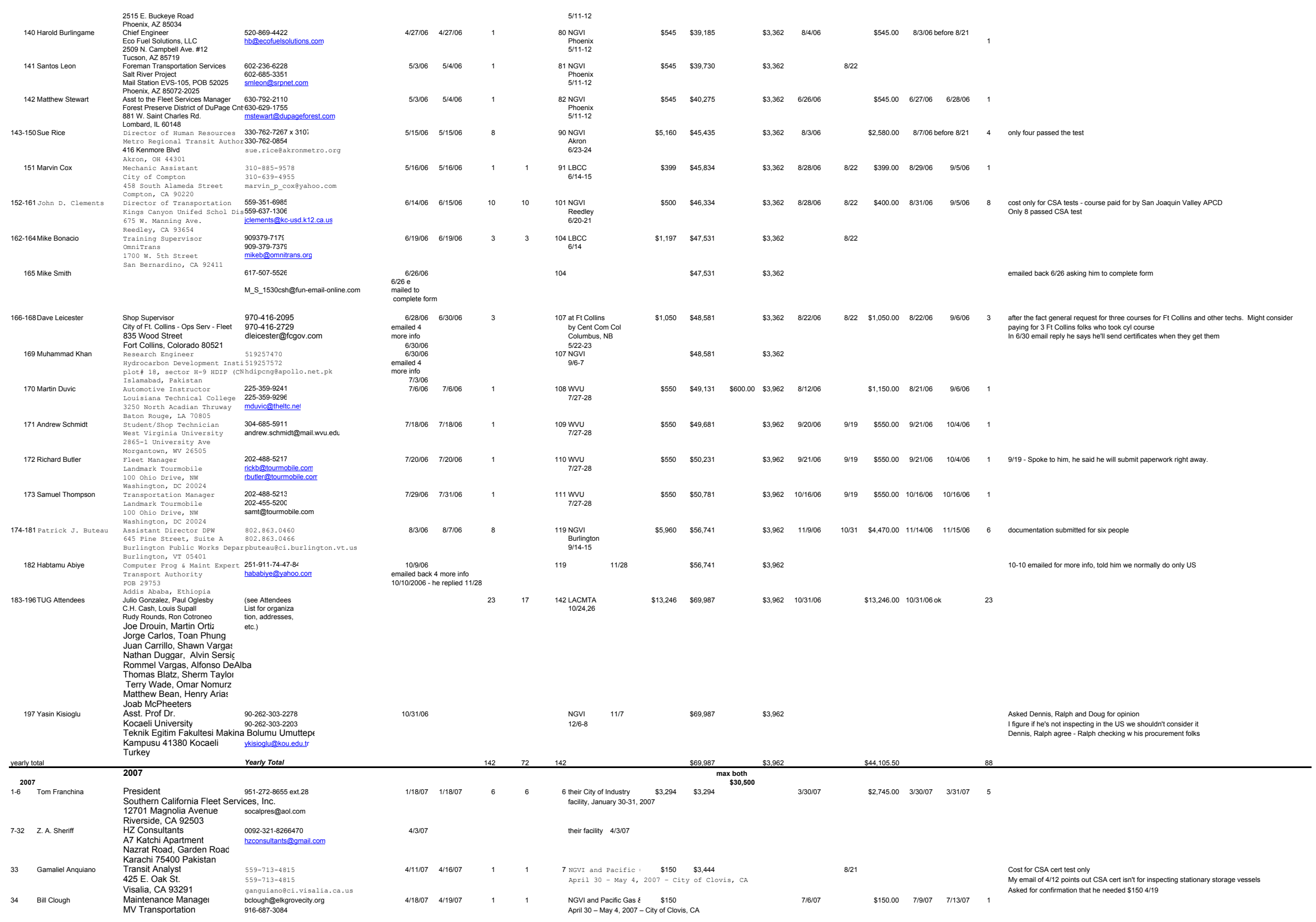




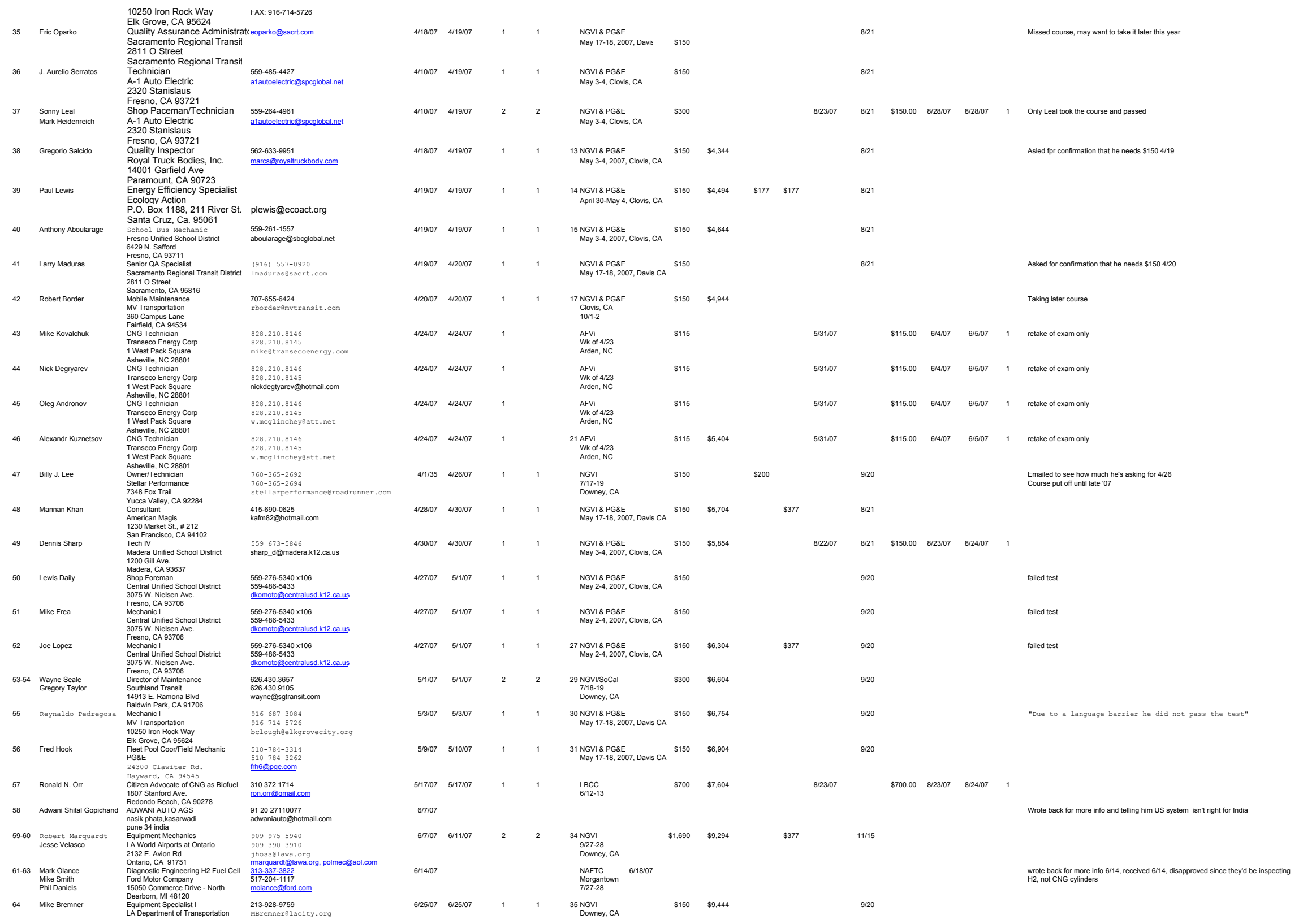




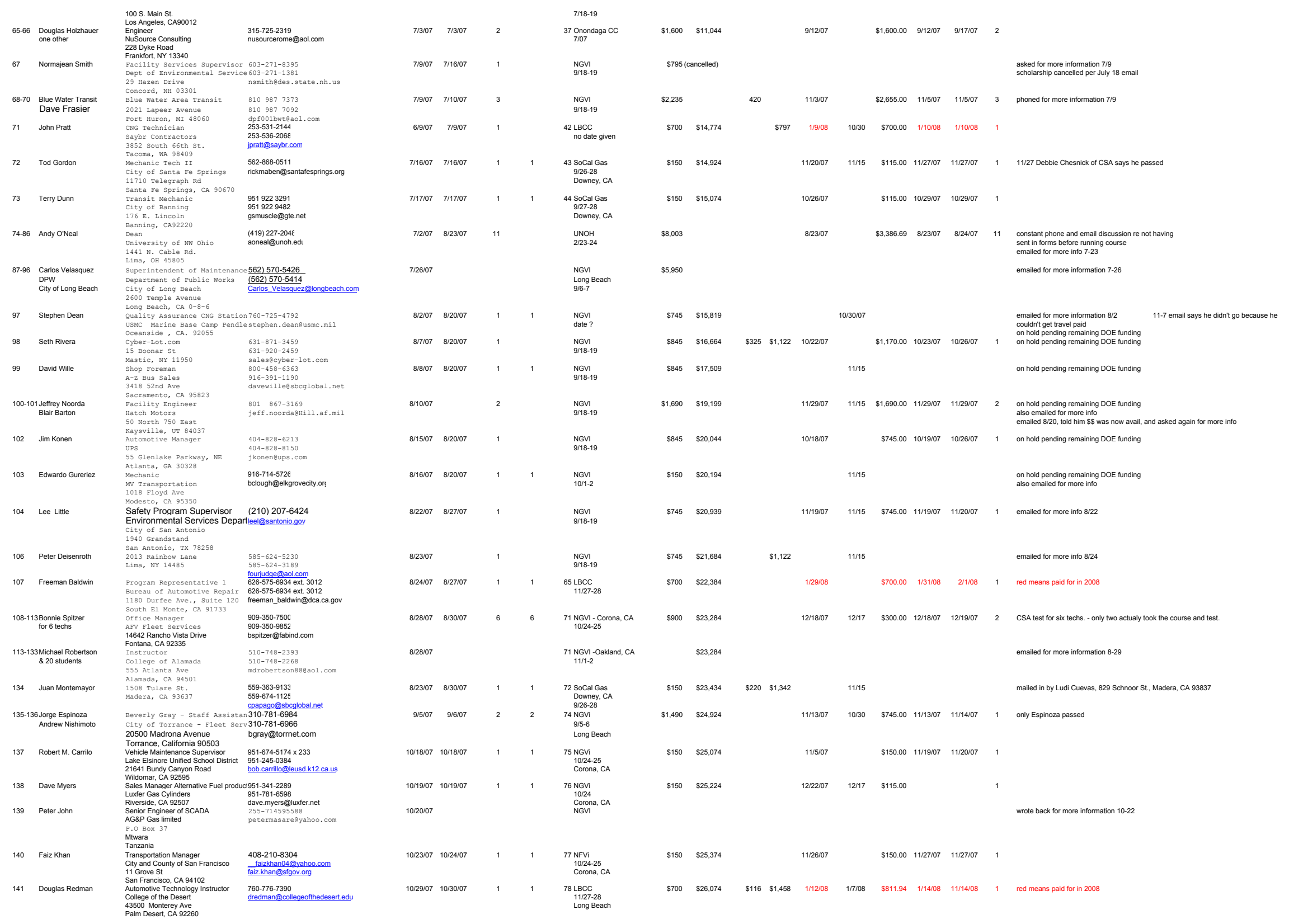




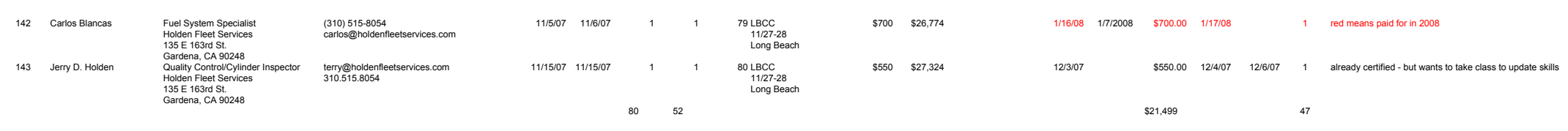

\begin{tabular}{|c|c|c|c|c|c|c|c|c|c|c|c|c|c|c|c|c|c|}
\hline & & 2008 & & & & & & & & & 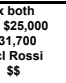 & & & & & & \\
\hline 1 & John Savacool & 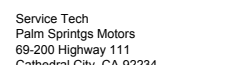 & $\begin{array}{l}760.965 .6020 \\
\text { jsavacoolepalmsp ringsmotors. com }\end{array}$ & 11/1007 & 2408 & 1 & 1 & $\begin{array}{l}1 \mathrm{NGVi} \\
11151.1708 \\
\text { Downey, CA }\end{array}$ & $\$ 150$ & $\$ 150$ & & NA & 50.00 & & & & Approved affer DOE funding - His co paid the total cost- closed \\
\hline 2 & James Madsen & 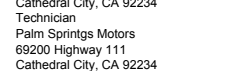 & $\begin{array}{l}760-328-2102 \\
\text { madsenis susteyahoo. com }\end{array}$ & 11124107 & 24108 & 1 & 1 & $\begin{array}{c}2 \mathrm{NGVi} \\
1115-17108 \\
\text { Downey, CA }\end{array}$ & $\$ 150$ & $\$ 300$ & & NA & & & & & Approved affer DOE funding - His co paid the total cost- closed \\
\hline 3-10 & Mark Pry & 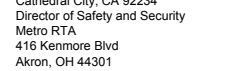 & 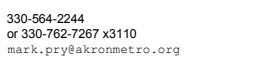 & 12/12/107 & 5/308 & 8 & & $\begin{array}{l}\text { Akron RTA } \\
\text { eary OB }\end{array}$ & $\$ 2,800$ & $\$ 3,100$ & & 12/31/1087/21 & $\$ 1,400.00$ & 124108 & & 4 & 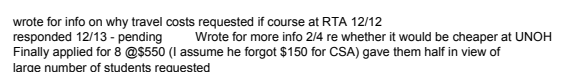 \\
\hline 11-14 & $\begin{array}{l}\text { Carlos Saldana } \\
\text { \& three others }\end{array}$ & 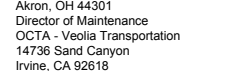 & $\begin{array}{l}\text { 949-857-7262 } \\
\text { FAX: } 949-857-7264 \\
\text { carlos.saldana@eveliatransportation.com }\end{array}$ & 4/16108 & 5/108 & 4 & 4 & $\begin{array}{l}\stackrel{L B C C}{ } \\
5 / 1 / 9-9\end{array}$ & $\$ 2,800$ & $\$ 5,900$ & & $7 / 21$ & & & & & 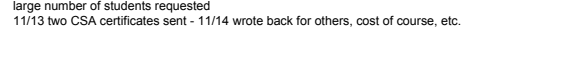 \\
\hline 15 & Kenny Pouncey & 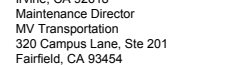 & 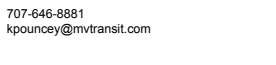 & 4/201/08 & 5/1108 & 1 & 1 & $\begin{array}{l}\mathrm{NGVi} \\
6 / 18-19\end{array}$ & $\$ 645$ & $\$ 6,545$ & & $8 / 21$ & & & & & 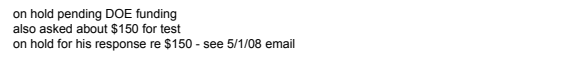 \\
\hline 16-17 & $\begin{array}{l}\text { Tim Kelly } \\
\text { and one other }\end{array}$ & 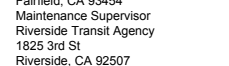 & 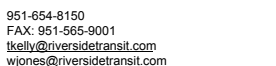 & $4 / 22208$ & 5/1108 & 2 & 2 & $\begin{array}{l}\mathrm{NGVi} \\
6 / 17-18\end{array}$ & $\$ 1,290$ & & & $7 / 16108$ & $\$ 1,290.00$ & 711708 & $7 / 21108$ & 2 & on hold pending DOE funding \\
\hline 18-20 & $\begin{array}{l}\text { Warren Riley } \\
\text { and two others }\end{array}$ & 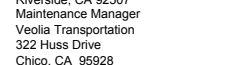 & 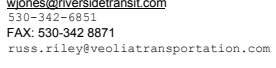 & 4/24108 & 5/1/108 & 3 & 3 & $\begin{array}{l}\mathrm{NGVi} \\
6 / 17-18\end{array}$ & $\$ 1,935$ & & $\$ 700$ & 8/121208 & $\$ 1,990.00$ & 811808 & $8 / 18108$ & 3 & on hold pending DOE funding \\
\hline 21 & Khandaker Ati qu & 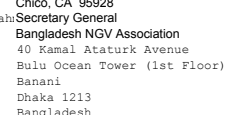 & 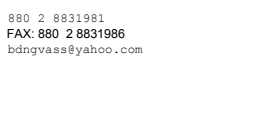 & 4266108 & & & & $\underset{6 / 17-18}{\operatorname{NGVi}} \quad 5 / 2$ & & & & & & & & & 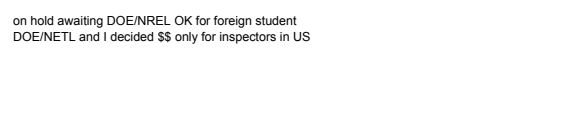 \\
\hline 22 & James $\mathrm{C}$. Herber & 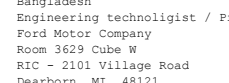 & 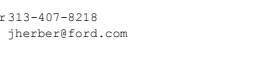 & $5 / 61685 / 7$ & & 1 & & $\begin{array}{l}\text { NAFTC } \\
\text { ArronRA } \\
\text { 5/15-16 }\end{array}$ & s950 & & & $8 / 4087121$ & $\$ 700.00$ & $8 / 408$ & $8 / 408$ & 1 & wrotet to see if he'd be inspecting $C N G$ or $\mathrm{H} 2$ cylinders \\
\hline 23 & Kurt Vassinder & 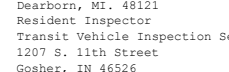 & $\begin{array}{l}\text { 57-534-4502 } \\
\text { evurmavas@everzonne }\end{array}$ & 577108519 & & 1 & & $\begin{array}{l}\text { NAFTC } \\
\text { ARTOPRTA } \\
\text { 515-16-16 }\end{array}$ & $\$ 700$ & & & 6/17708 & $\$ 700.00$ & 6/1908 6 6119 & & 1 & \\
\hline 24 & George Vasbinder & 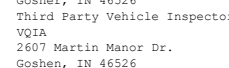 & $\begin{array}{l}\text { r574-536-083 } \\
\text { vqia@maplenet.ne }\end{array}$ & $5 / 51085 / 9$ & & 1 & & $\begin{array}{l}\text { NAFTC } \\
\text { AFronRA } \\
\text { 51/5-16 }\end{array}$ & $\$ 700$ & & & 6/17708 & $\$ 700.00$ & 6/191086 6/19 & & 1 & \\
\hline 25 & Vince Denman & 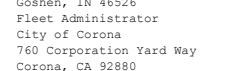 & $\begin{array}{l}\begin{array}{l}951-736-2306 \\
\text { FAX } 951-279-350 \ell \\
\text { vince.denman@icorona.ca.us }\end{array} \\
\end{array}$ & 5/1208 & & & & $\begin{array}{l}\text { NGVi } \\
\text { Luxier } \\
66 / 7-18\end{array}$ & & & & & & & & & $\begin{array}{l}\text { don't want to take test, wont' be inspecting enough } \\
\text { therefore scholarship refused }\end{array}$ \\
\hline 26-28 & $\begin{array}{l}\text { Jason Hohalek } \\
\text { and } 2 \text { ofhers }\end{array}$ & 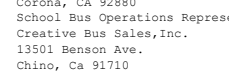 & $\begin{array}{l}\text { eq09-465-5528 } \\
\text { jasonhecreativebussales.com }\end{array}$ & $5 / 81085 / 1$ & & 3 & 3 & 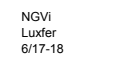 & $\$ 1,935$ & & & $8 / 21 / 088 / 21$ & $\$ 1,290.00$ & 8/21108 & & 2 & 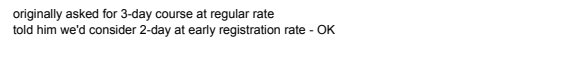 \\
\hline 29.44 & $\begin{array}{l}\text { Tom Sewell } \\
\text { and 11-14 others }\end{array}$ & 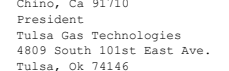 & $\begin{array}{l}\text { (918) } 665-2641 \\
\text { tseeve11etulsagastech. com }\end{array}$ & $5 / 9108$ & & & & & & & & & & & & & wrote back on $5 / 12$ for more information \\
\hline 45 & George Wofford & 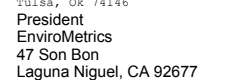 & 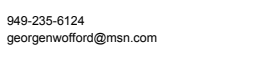 & 5/13/108 5/1/1 & & 1 & 1 & $\begin{array}{l}\text { NoVi } \\
\text { Luxder } \\
6417-18\end{array}$ & $\$ 645$ & & & $8 / 21$ & & & & & \\
\hline 46 & Leon Schrader & 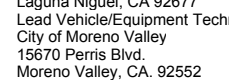 & 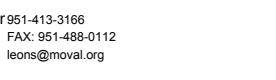 & 5/9/988 5/2 & & 1 & 1 & $\begin{array}{l}\text { NGVi } \\
\text { Luxder } \\
6417-18\end{array}$ & $\$ 645$ & & & 8/29/1088/21 & $\$ 645.00$ & 9/208 & 9/3/108 & 1 & \\
\hline 47 & Larry Hisel & 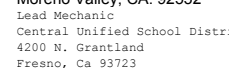 & 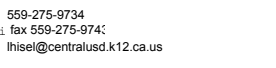 & 5/9/9/85 5/2 & & 1 & 1 & 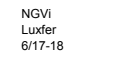 & $\$ 645$ & & $\$ 300$ & $8 / 21$ & & & & & \\
\hline 48 & Mike Frea & 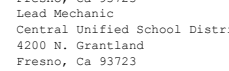 & 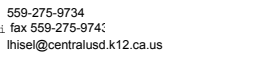 & 5/9/9/18 5/2 & & 1 & 1 & $\begin{array}{l}\text { NGVi } \\
\text { Luter } \\
6 / 17-18\end{array}$ & $\$ 645$ & & $\$ 300$ & $8: 21$ & & & & & \\
\hline 49 & Thomas Henry & 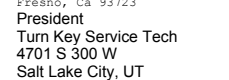 & $\begin{array}{l}\text { 801-261-8034 } \\
\text { tomandobobi@xmission. com }\end{array}$ & $5 / 20085$ 5/2 & & 1 & & 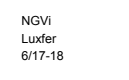 & $\$ 645$ & & & 8/2221088/21 & $\$ 645.00$ & $8 / 2508$ & 8126108 & 1 & \\
\hline 50-52 & $\begin{array}{l}\text { Keith Delk } \\
\text { and two others }\end{array}$ & $\begin{array}{l}\text { Manager, Product Support } \\
\text { Haaker Equipment Co. } \\
\text { 2070 N. White Aue. } \\
\text { La Verne, CA 91750 }\end{array}$ & $\begin{array}{l}\text { 909-598-2706 } \\
\text { keitddahaker.com }\end{array}$ & 5/201085/2 & & 3 & 3 & $\begin{array}{l}\text { NGVi } \\
\text { Luxter } \\
6 / 77-18\end{array}$ & $\$ 1,935$ & & & $8 / 21$ & & & & & \\
\hline
\end{tabular}




\begin{tabular}{|c|c|c|c|c|c|c|c|c|c|c|c|c|c|c|c|c|}
\hline 53 & Michael Millet & $\begin{array}{l}\text { Profoessor } \\
\text { Salt Lake Community College } \\
4556 \text { south } 3245 \text { west }\end{array}$ & $\begin{array}{l}\text { 801-244-6453 } \\
\text { mike.millet@sclc.edu }\end{array}$ & $5 / 211085522$ & 1 & & $\begin{array}{l}\text { Novi } \\
\text { Luxter } \\
6 / 17-18\end{array}$ & $\$ 645$ & $\$ 300$ & 8/2 & & & & & & 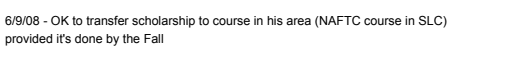 \\
\hline 54 & Buck Bellower & $\begin{array}{l}\text { Buck Belf Ilover } \\
\text { Mechanic } \\
\text { city of Lindsay } \\
\text { PoB } 369\end{array}$ & 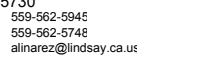 & $5 / 211085523$ & 1 & 1 & $\begin{array}{l}\text { NGVi } \\
\text { Luxter } \\
6 / 77-18\end{array}$ & $\$ 645$ & $\$ 300$ & 10/14/1088/2 & & $\$ 945.00$ & 10/4408 & 10171708 & 1 & wrote back on $5 / 22$ for more intromation \\
\hline 55 & Joe Makovics & 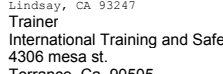 & $\begin{array}{l}\text { 310-989-2165 } \\
\text { e jomak1@verizon.net }\end{array}$ & 5/22085 5/22 & 1 & 1 & $\begin{array}{l}\text { Nuvi } \\
\text { Luxter } \\
6 / 17-18\end{array}$ & $\$ 645$ & & $8 / 2$ & & & & & & \\
\hline $56-57$ & $\begin{array}{l}\text { Doug Spencer } \\
\text { and one other }\end{array}$ & 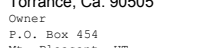 & $\begin{array}{l}\text { douglas.jspencer@us.army.mi } \\
435-462-0132\end{array}$ & 5/2808 55/29 & 2 & seen note & $\begin{array}{l}\text { NGVi } \\
\text { Luxter }\end{array}$ & $\$ 1,490$ & & $\begin{aligned} 8 / 2108 \\
8 / 2308\end{aligned}$ & & $\begin{array}{l}\$ 745.00 \\
\$ 745.00\end{array}$ & $\begin{array}{r}8 / 4108 \\
8 / 25108\end{array}$ & $\begin{array}{l}8 / 12108 \\
8 / 26108\end{array}$ & 1 & 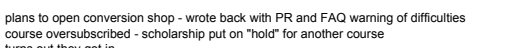 \\
\hline 58-61 & $\begin{array}{l}\text { Encic Mabey } \\
\text { and three others }\end{array}$ & 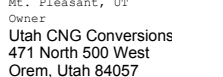 & $\begin{array}{l}\text { utahnongconversions@gmail.com } \\
801-368-8380 \\
\text { fax 801-225-4029 }\end{array}$ & $5 / 2908066 / 2$ & 4 & & 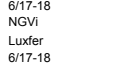 & $\$ 2,980$ & $\$ 300$ & 1291908 & $8 / 21$ & $\$ 3,280.00$ & 1219108 & 1210108 & 4 & furns out they gotin \\
\hline 62 & $\begin{array}{l}\text { Daniel Dy } \\
\text { and one other }\end{array}$ & 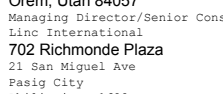 & $\begin{array}{l}\text { is danny. dy } 2003 \text { egma i } 1 \text {.com } \\
6326873750\end{array}$ & 6/2/208 & & & $\begin{array}{l}\text { NGVi } \\
\text { Luxier } \\
6 / 17-18\end{array}$ & & & & & & & & & \\
\hline 63 & william Hewitt & $\begin{array}{l}\text { Philitippines } 16000 \\
\text { Mechanic } \\
\text { Harper Redi Mix } \\
5442 \text { S. Tropicana Dr. }\end{array}$ & $\begin{array}{l}\text { 801-455-3645 } \\
\text { malloygacambriapm.com }\end{array}$ & $6 / 11108$ & & & $\begin{array}{l}\text { NAFTC } \\
\text { SLC } \\
\text { July o8 }\end{array}$ & & & & & & & & & 6112 wrote back telling him we only pay for inspector course \& a sking for more intormation \\
\hline 64 & Josh Cummings & 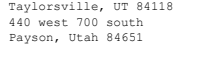 & $\begin{array}{l}435621499 \mathrm{C} \\
\text { cumni } 195570 \text { eho tma i } 1 . \text { com }\end{array}$ & 6/1208 & & & $\begin{array}{l}\text { NAFTC } \\
\text { SLC } \\
\text { July yo }\end{array}$ & & & & & & & & & $6 / 13$ wrote back telling him we only pay for inspector course \& a asking for more information \\
\hline 65 & Karl Haten & $\begin{array}{l}476 \text { S } 500 \text { E } \\
\text { St George UT } 84770\end{array}$ & 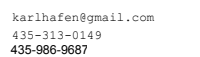 & 61151086 6/16 & 1 & & $\begin{array}{l}\text { NAFC } \\
\text { NLC } \\
\text { July }\end{array}$ & $\$ 650$ & $\$ 300$ & 9/25108 & 9/16 & $\$ 950.00$ & 9/25/08 & & 1 & 616 wrote back telling him we only pay for inspector course \& a sking for more information \\
\hline 66 & Stanley Martineau & $\begin{array}{l}\text { Manager } \\
\text { SVM LIC } \\
\text { POB } 116\end{array}$ & stan.martineau@cuu.edt & 61661086617 & 1 & & $\begin{array}{l}\text { NAFTC } \\
\text { SLI } \\
\text { Juyg-11 }\end{array}$ & $\$ 650$ & $\$ 300$ & $9 / 22108$ & 9/16 & $\$ 9950.00$ & 9/23308 & 9/24/08 & 1 & 616 wrote back telling him we only pay for inspector course \& a skxing for more intrormation \\
\hline $67-68$ & $\begin{array}{l}\text { Chares Flowers } \\
\text { and one other }\end{array}$ & 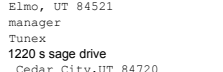 & 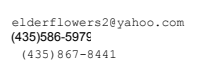 & 611610886/24 & 1 & & $\begin{array}{l}\text { NAFTC } \\
\text { SLC } \\
\text { July } 9-11\end{array}$ & $\$ 650$ & & 9/1808 & 9/16 & $\$ 650.00$ & 9/18108 & 9/19/08 & 1 & $6 / 16$ wrote back telling him we only pay for inspector course \& a sking for more information \\
\hline 69 & Stanley Witt & $\begin{array}{l}\text { President CNG Conversions, Inc. } \\
83 \mathrm{~W} 2350 \mathrm{~N}\end{array}$ & $\begin{array}{l}.801-450-4222 \\
801-5251-1008 \\
\text { switcoconversions com }\end{array}$ & 61161086/19 & 1 & & 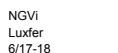 & $\$ 645$ & $\$ 300$ & 9/12/108 & $8 / 21$ & $\$ 920.00$ & 9/15/08 & 9/16/108 & 1 & $\begin{array}{l}\text { 6/17 w wotet back for more information - from their website it appears they don't use } \\
\text { EPA approved conversion kits }\end{array}$ \\
\hline 70 & Jim Atkinson & 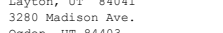 & 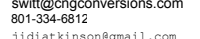 & 6/16/108 & & & & & & & & & & & & 6177 wrotet back tor more intormation on their plans 10 do conversions \\
\hline 71 & Mark Anderson & $\begin{array}{l}\text { Technical Specialist } \\
\text { Utan County Bureau of Air Qua } \\
3255 \mathrm{~N} \text { Main }\end{array}$ & 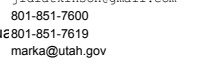 & 6177086 6/19 & 1 & & $\begin{array}{l}\mathrm{NGVi} \\
8 / 26-27\end{array}$ & $\$ 650$ & & & 9/16 & & & & & \\
\hline 72 & Anthur Andrew Scolson & 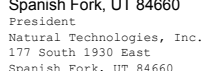 & $\begin{array}{l}801-319-6316 \\
\text { naturalechnologies@gmail.corn }\end{array}$ & 6177086619 & 1 & & $\begin{array}{l}\text { NAFTC } \\
\text { SLC }\end{array}$ & $\$ 650$ & & 10/6/108 & 9/16 & $\$ 650.00$ & 1016/108 & 10/7108 & 1 & 6/19 wrote back for more intrormation \\
\hline 73 & Damin Barbieri & 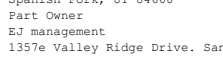 & 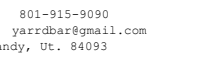 & 6177/086/23 & 1 & & $\begin{array}{l}\text { NAFTC } \\
\text { SLC } \\
\text { July } 9-11\end{array}$ & $\$ 650$ & & 9/2408 & 9/16 & $\$ 650.00$ & 9/25/08 & 9/25/108 & 1 & $6 / 19$ wrote back for more information \\
\hline 74 & Melvin Nensen & 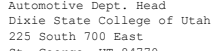 & jensenm@dixie.edt & 61777086/19 & 1 & & $\begin{array}{l}\text { NAFTC } \\
\text { SLC }\end{array}$ & $\$ 650$ & $\$ 300$ & & 9/16 & & & & & \\
\hline 75 & Mark Hatch & $\begin{array}{l}\text { S.t. George, UT } 847700 \\
\text { OHaner } \\
\text { Hatch Motors } \\
\text { 3ato so Fort Lane } \\
\text { Thon }\end{array}$ & 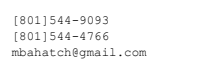 & 6/1180868/24 & & & $\begin{array}{l}\text { July y-11 } \\
\text { NAFC } \\
\text { sul } \\
\text { July } 14-18\end{array}$ & $\$ 650$ & & & & & & & & 6/19 wrote back for more intrormation \\
\hline 76 & Norman Larsen & 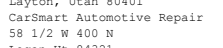 & $\begin{array}{l}\begin{array}{l}435-512-1333 \\
\text { chevcarsmartehotmai } 1 . \text { com }\end{array}\end{array}$ & 6/181808 & & & $\begin{array}{l}\text { NAFTC } \\
\text { SLC }\end{array}$ & & & & & & & & & 6/19 wrote back for more information \\
\hline 77 & Jim Hare & 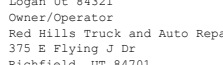 & 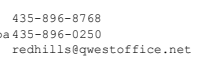 & 614818086/23 & 1 & & $\begin{array}{l}\text { S } \\
\text { SATC } \\
\text { July } 14-18\end{array}$ & $\$ 650$ & & & 9/16 & & & & & $6 / 19$ wrote back for more initorm \\
\hline $78-79$ & $\begin{array}{l}\text { Jeff Donovan } \\
\text { Al Boman }\end{array}$ & 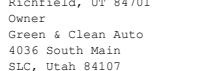 & $\begin{array}{l}801-541-5730 \\
\text { jsdonovane concast. net }\end{array}$ & 6/199086/19 & 2 & & $\begin{array}{l}\text { NAFTC } \\
\text { SLCC }\end{array}$ & $\$ 1,300$ & & 9/15/08 & & $\$ 1,300.00$ & 9/16/108 & 9/16/108 & 2 & $6 / 19$ wrote back for more information \\
\hline 80 & Craig Jorgensen & $\begin{array}{l}\text { Air Pollution Control Auditor } \\
\text { Weber-morgan Health Dept. } \\
477723 \text { drd Stret } \\
\text { Ogaden UT UT } 84401\end{array}$ & $\begin{array}{l}\text { 801-399-7140 } \\
\text { 801-199-9145 } \\
\text { ciorgensen@co.weber.ut.us }\end{array}$ & 6/199086/19 & 1 & & $\begin{array}{l}\text { NAFTC } \\
\text { SLC } \\
\text { July 14-18 }\end{array}$ & $\$ 650$ & & 9/17/08 & 9/16 & $\$ 650.00$ & 9/18/08 & 9/16/108 & 1 & \\
\hline 81 & Matt cook & 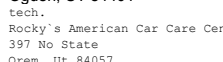 & $\begin{array}{l}801-655-1144 \\
\text { nntgti remanhenotmai1 . com }\end{array}$ & 6/19/108 & & & $\begin{array}{l}\text { NAFTC } \\
\text { SLC } \\
\text { July 14-18 }\end{array}$ & & & & & & & & & 6/23 wrote back for more information \\
\hline 82 & Kimball Phillips & 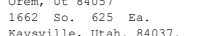 & $\begin{array}{l}801-550-3959 \\
k\end{array}$ & 6/20108 & & & $\begin{array}{l}\text { NAFTC } \\
\text { SIC }\end{array}$ & & & & & & & & & $6 / 23$ wrote back for more information \\
\hline 83 & Shawn Horrocks & 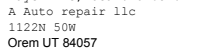 & 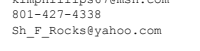 & 6/23108 & & & $\begin{array}{l}\text { NAFTC } \\
\text { SLC }\end{array}$ & & & & & & & & & $6 / 23$ wrote back for more informatio \\
\hline 84 & Giovanni Guanuna & $\begin{array}{l}\text { Managere cout } \\
\text { Auto Gas conversion } \\
4768 \text { summerwood Dr. }\end{array}$ & $\begin{array}{l}\text { 801-652-8462 } \\
\text { giovanni86_9emsn.com }\end{array}$ & 6/24008 6/26 & 1 & & $\begin{array}{l}\text { NAFTC } \\
\text { SLC } \\
7 / 28-8 / 1\end{array}$ & $\$ 650$ & & & 9/16 & & & & & 6/24 wrott back for more information \\
\hline 85 & Tai Robinson & $\begin{array}{l}\text { President- - Chief Technology C } \\
\text { Intergalactic Hydrogen } \\
9851 \text { S. Borg Dr. }\end{array}$ & $\begin{array}{l}\text { C801-201-7370 } \\
\text { tai@n290.info }\end{array}$ & $6 / 240868124$ & 1 & & $\begin{array}{l}\text { NAFTC } \\
\text { SLC }\end{array}$ & $\$ 650$ & & $117 / 108$ & $9 / 16$ & $\$ 650.0 \mathrm{c}$ & 11/10/08 & 11/144/08 & 1 & \\
\hline
\end{tabular}




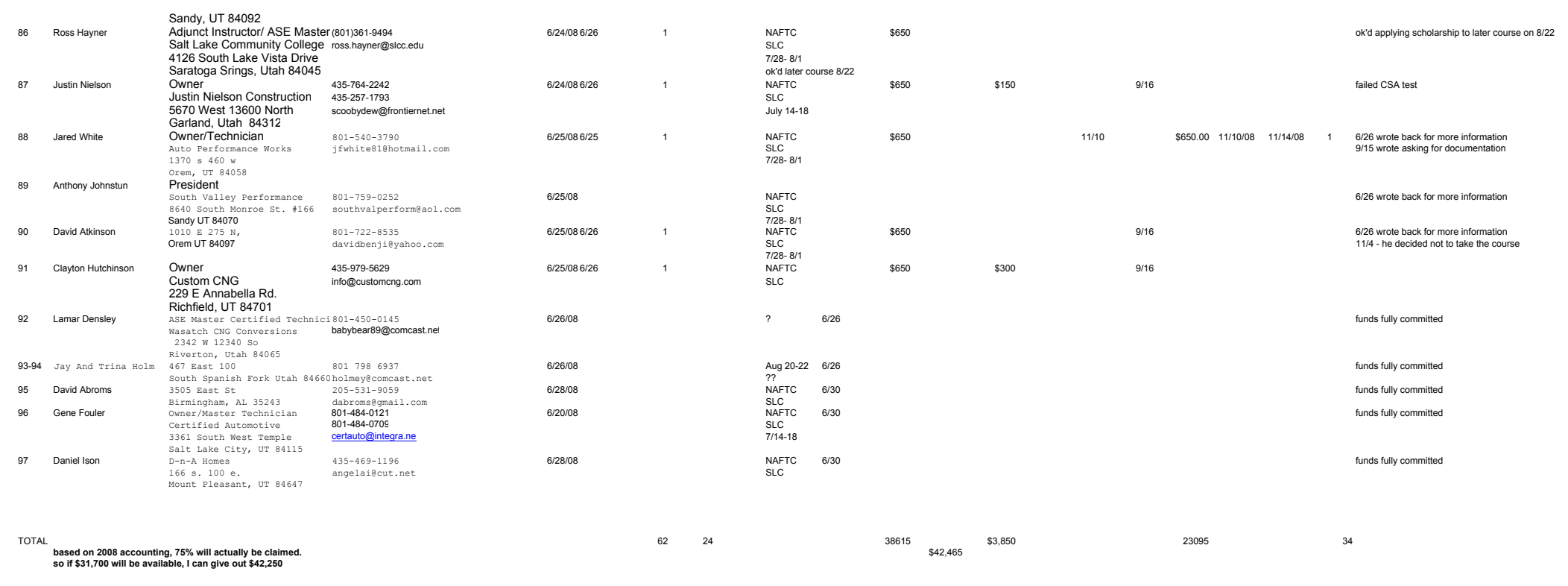




\section{Appendix I - CNG Fuel System Inspector}

\section{Study Guide}

Sponsored by:

National Energy Technology Laboratory

U.S. Department of Energy

DE-FC26-05NT42608

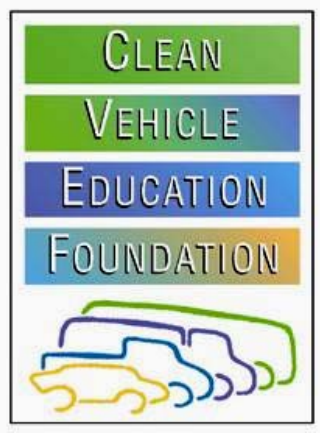

Contract Manager:

Clean Vehicle Education Foundation

Point of Contact: Doug Horne

President

770-424-8575

FAX: 770-424-8575

dbhorne@cleanvehicle.org

Submitted by:

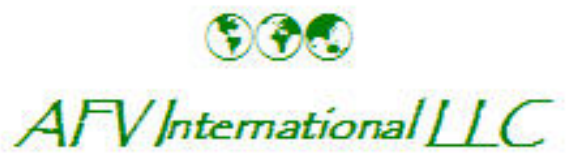




\section{Acknowledgments}

It is a privilege to work on such a relevant and exciting effort as this. Energy independence, environmental, and technology transfer issues are at the heart of a dynamic and mobile society and were at the heart of the project.

Safety issues associated with the health and quality of the natural gas vehicle industry were the initial goals of the project. But a slightly different, perhaps more profound spirit emerged as we began our work. We soon came to recognize a higher purpose to support and encourage a 'movement' to participate in a larger mission, improving an industry that can significantly impact our world. This movement comprised individuals and companies, loosely organized advocates, officials, technical experts, and practitioners - each contributing a positive energy to help place alternative fuels higher on America's agenda.

The material presented in this study guide could not have been collected without the cooperation of numerous alternative fuel industry representatives, committees, and individuals. From these sources, technical details and graphic examples were always forthcoming. Time was freely given to review text or participate in development sessions.

The funding to develop this material came from a grant from the U.S. Department of Energy, under the leadership of Mr. Hank Seiff, Technical Director for the Clean Vehicle Education Foundation.

Additional support came from the United States standards development organization CSA America, Inc. Spearheading this effort was Mr. Mike Dickerson, Product Manager, Personnel Certification. With his patient guidance throughout the process we were able to complete a rigorous series of meetings and numerous conference calls culminating in a well developed examination and certification program for the CNG Fuel System Inspector.

The following individuals and institutions contributed significantly to this text: Clean Vehicle Education Foundation Long Beach Community College Advanced Technology Training Centers Mr. Hank Seiff Mr. Cal Macy Mr. Peter Davis

To these and all of those who participated in this project, thank you for your efforts and we hope you will find the result a contribution to your good work.

William H. 'Bill' McGlinchey

AFV International LIc

Lancaster, $\mathrm{OH}$

(740) 205-2107

w.mcglinchey@att.net 


\section{About CSA America}

CSA America, Inc. is well known as the standards writing body in the United States for gas appliances and accessories and alternative energy products. It had its origins in this country as the American Gas Association Labs. Now they are part of CSA-International with laboratories all over the country. The offices for this program are in Cleveland, $\mathrm{OH}$.

CSA America Inc.

8501 East Pleasant Valley Road

Cleveland, $\mathrm{OH} 44131-5575$

Tel: (216) 524-4990

Fax: (216) 520-8979

http://csa-america.org

In 2006 CSA America assumed the administration of the CNG Cylinder Inspector Certification program from CSA International with the goal of strengthening the content, administration, systems and procedures. Since then, CSA America has been working to update and improve the CNG Cylinder Inspector Certification program. The new program was made available August 1, 2008 as the CNG Fuel System Inspector Certification program and follows the International Organization for Standardization (ISO) 17024 Conformity Assessment standard, the global benchmark for personnel certification programs. 


\section{About the Exam}

The exam represents the conclusion of two years of development, several workshops convened across the country and many more conference calls among a group of dedicated industry professionals. The CNG Fuel System Inspector Certification is on a three year re-examination cycle. Those CNG Cylinder Inspectors certified under the older version (prior to 8/1/2008) of the test will have to retest at the expiration of their CNG Cylinder Certification to re-certify in the expanded certification program. In nonexamination years there will be an annual administration fee to maintain your certification. Unlike the previous program, there will be no minimum cylinder or vehicle inspection requirements to meet in order to renew your CNG Fuel System Inspector Certification.

The CSA test contains approximately sixty multiple choice questions. There may be additional test questions included that are for evaluation purposes only and will not be part of the final scoring. The questions are based on expert opinions from a cross section of the CNG industry after consideration of the skills and knowledge that a minimally qualified applicant should have. They are taken in proportion to their importance from a carefully constructed set of objectives or tasks that inspectors would be expected to perform.

In preparation for taking the exam, you should first evaluate yourself against these objectives. Honestly consider if you are confident that you know each specific task listed in the following Task List. Note the percentage of questions you can expect in each category and check Yes $(\mathrm{Y})$ or No $(\mathrm{N})$ as you grade yourself. This will form the basis for any additional study you need prior to taking the test.

You can learn more about registration requirements, fees, qualifications and training resources from the CSA America website:

http://csa-america.org/personnel certification/cng certification/default.asp?load=getcert

Appendix $D$ has more about taking the test and a sampling of test questions for your review.

Good luck and study hard.

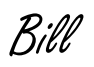




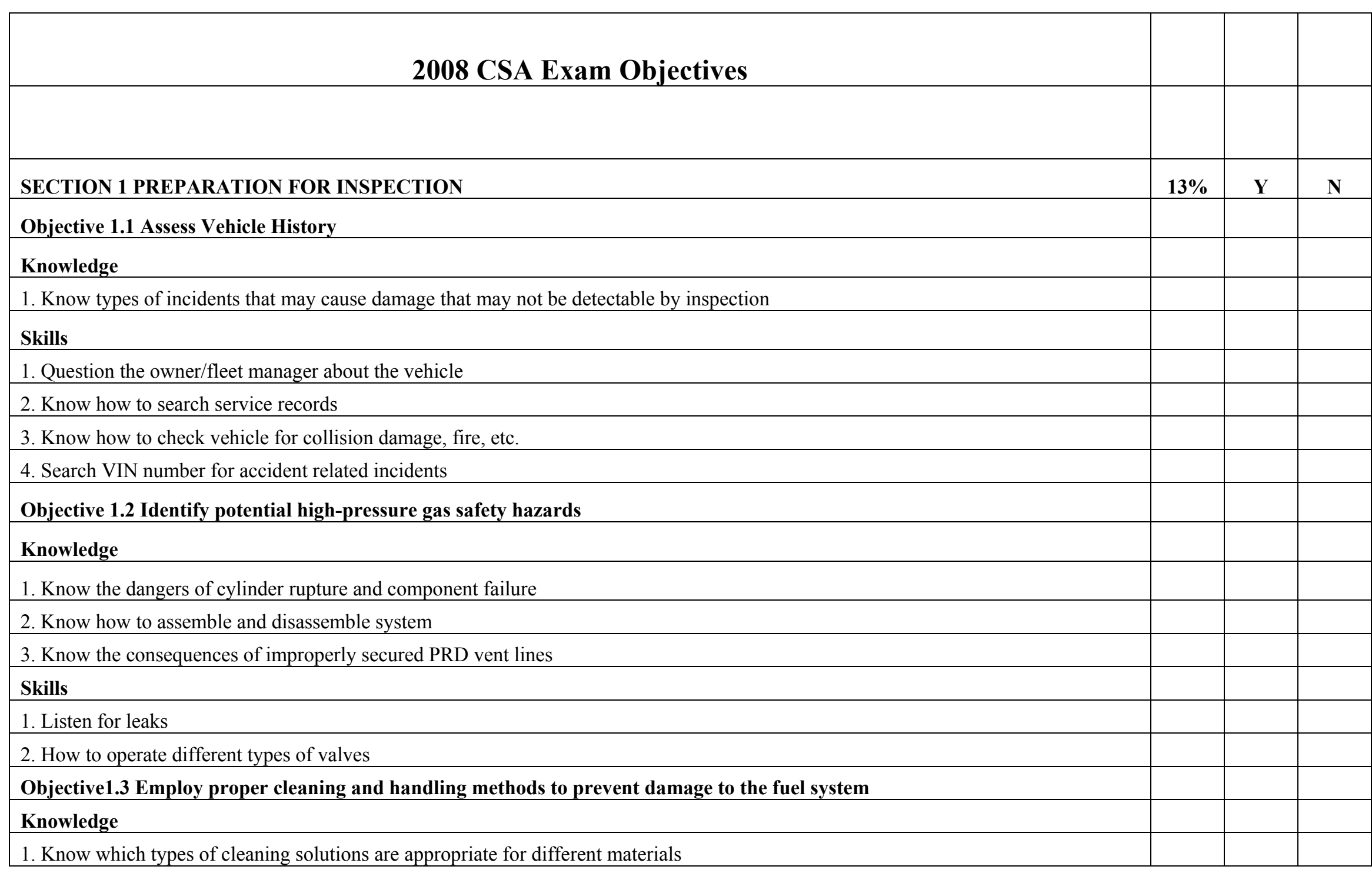




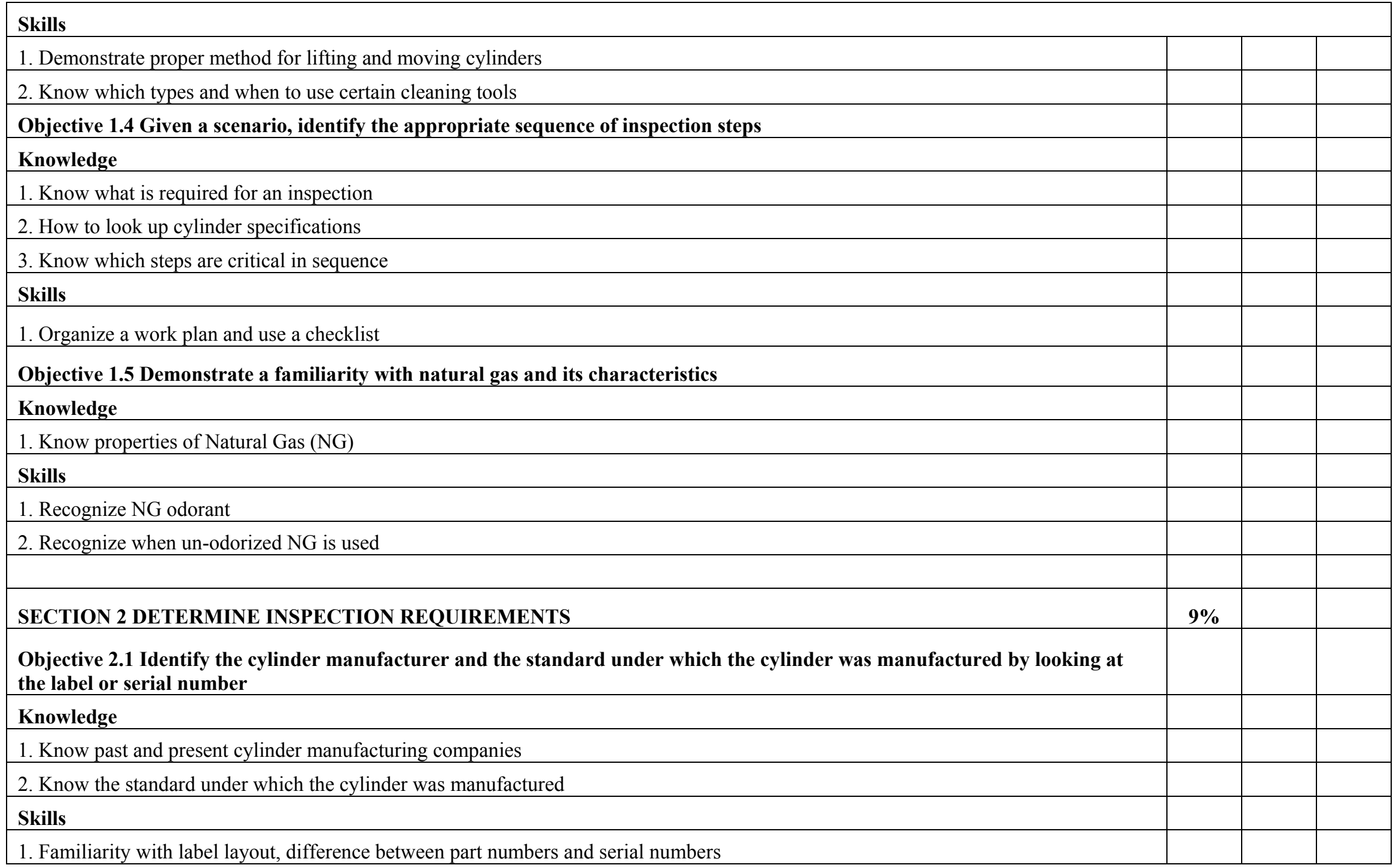


Objective 2.2 Select the proper inspection standard and/or the manufacturer's inspection recommendations based on the label

Knowledge

1. Familiarity with inspection standards

2. Know if there is a manufacturers' standard that's applicable to the vehicle

3. Know that the manufacturers' standard always goes first

Skills

1. Ability to interpret the standards

Objective 2.3 Determine which NFPA 52 requirements apply to the vehicle being inspected

Knowledge

1. Know the dates of the past NFPA 52 revisions

2. Know how to recognize the labeling of an OEM certified vehicle vs. an aftermarket conversion Skills

1. How to correlate the vehicle with NFPA 52

Objective 2.4 Verify that the cylinder inspection documents are appropriate to the vehicle

Knowledge

1. Know the dates of the past inspection document revisions

2. Know how to recognize the labeling of an Original Equipment Manufacturer (OEM) certified vehicle vs. an aftermarket conversion Skills

1. How to correlate your vehicle with the inspection documents

Objective 2.5 Know the sources of additional inspection information and explain where to find them Knowledge

1. Know the cylinder, component, system, vehicle manufacturers

Skills

1. Accessing contact information

\begin{tabular}{|l|l|l|}
\hline & & \\
\hline & & \\
\hline & & \\
\hline & & \\
\hline & & \\
\hline & & \\
\hline & & \\
\hline & & \\
\hline & & \\
\hline & & \\
\hline & & \\
\hline & & \\
\hline & & \\
\hline & & \\
\hline & & \\
\hline & & \\
\hline
\end{tabular}




\section{SECTION 3 PRESSURE RELIEF DEVICE (PRD) INSPECTION}

Objective 3.1 Given a scenario, inspect PRD piping for damage, obstructions, restrictions and verify that it is properly seated to prevent foreign material from entering.

Knowledge

1. Know what a venting system looks like

2. Know function and appearance of venting systems

3. Know the different types of PRD channel configurations

Skills

1. Recognize different types of damage

2. Recognize probable modifications

Objective 3.2. Identify visible damage and assess serviceability of PRD's and verify that the PRD has not been recalled Knowledge

1. Know how to recognize a PRD

2. Know the location of PRD's

3. Know lists of manufacturers

Skills

1. Know how to find the list of recalled PRD's

Objective 3.3 Recognize if the PRD vent line is properly routed, supported and adequate for venting pressure Knowledge

1. Know what a good PRD looks like

Skills

1. Be able to differentiate between original manufacturer versus later extrusion of the eutectic trigger

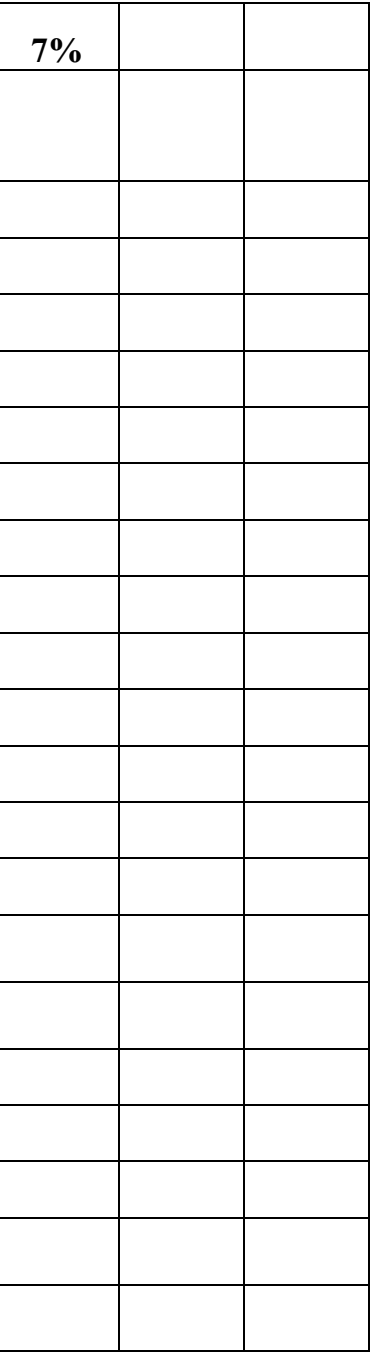




\section{SECTION 4.0 PHYSICAL INSPECTION OF BRACKETS AND HIGH-PRESSURE COMPONENTS}

Objective 4.1 Identify missing components or damage to guards and covers that could affect cylinder or system integrity

Knowledge

1. Know in which situations guard covers are required

2. Know different designs of guards and covers

Skills

1. Rubber gaskets and clearance requirements

\section{Objective 4.2 Assess the condition of CNG cylinder mounting systems}

Knowledge

N/A

\section{Skills}

1. Know how to identify systems that have been degraded

2. How to identify missing or out of place rubber isolation gaskets

3. How to identify over tightened brackets

3. How to identify misaligned brackets

Objective 4.3 Identify CNG fuel system components and describe their functions

Knowledge

1. Know the major components

2. Know the function of each component based on its appearance and location in the system Skills

1. Recognize defective, damaged or missing components

Objective 4.4 Recognize system defects that would require a partial or full system defueling

\begin{tabular}{|l|l|l|}
\hline $\mathbf{1 8 \%}$ & & \\
\hline & & \\
\hline & & \\
\hline & & \\
\hline & & \\
\hline & & \\
\hline & & \\
\hline & & \\
\hline & & \\
\hline & & \\
\hline & & \\
\hline & & \\
\hline & & \\
\hline
\end{tabular}




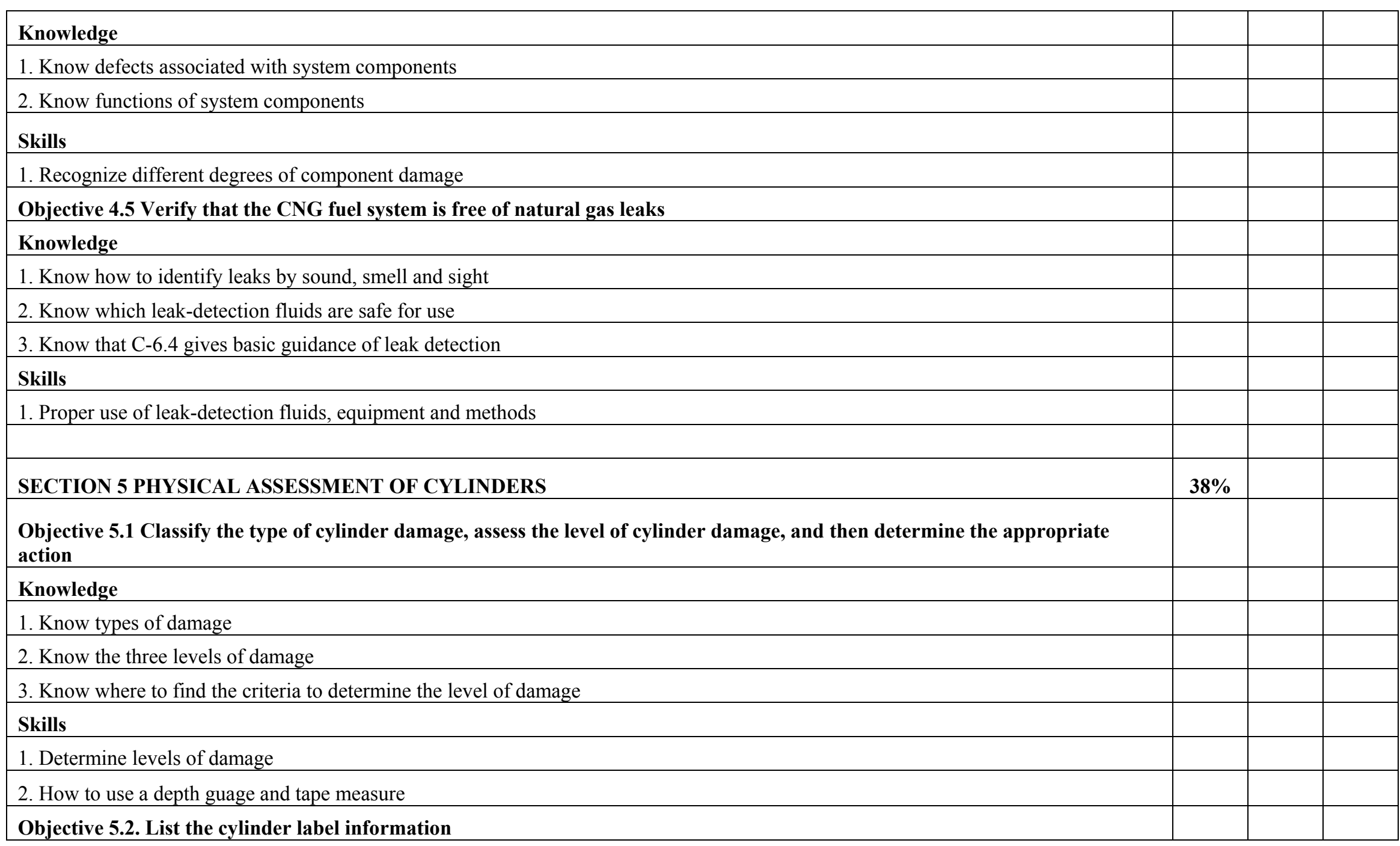




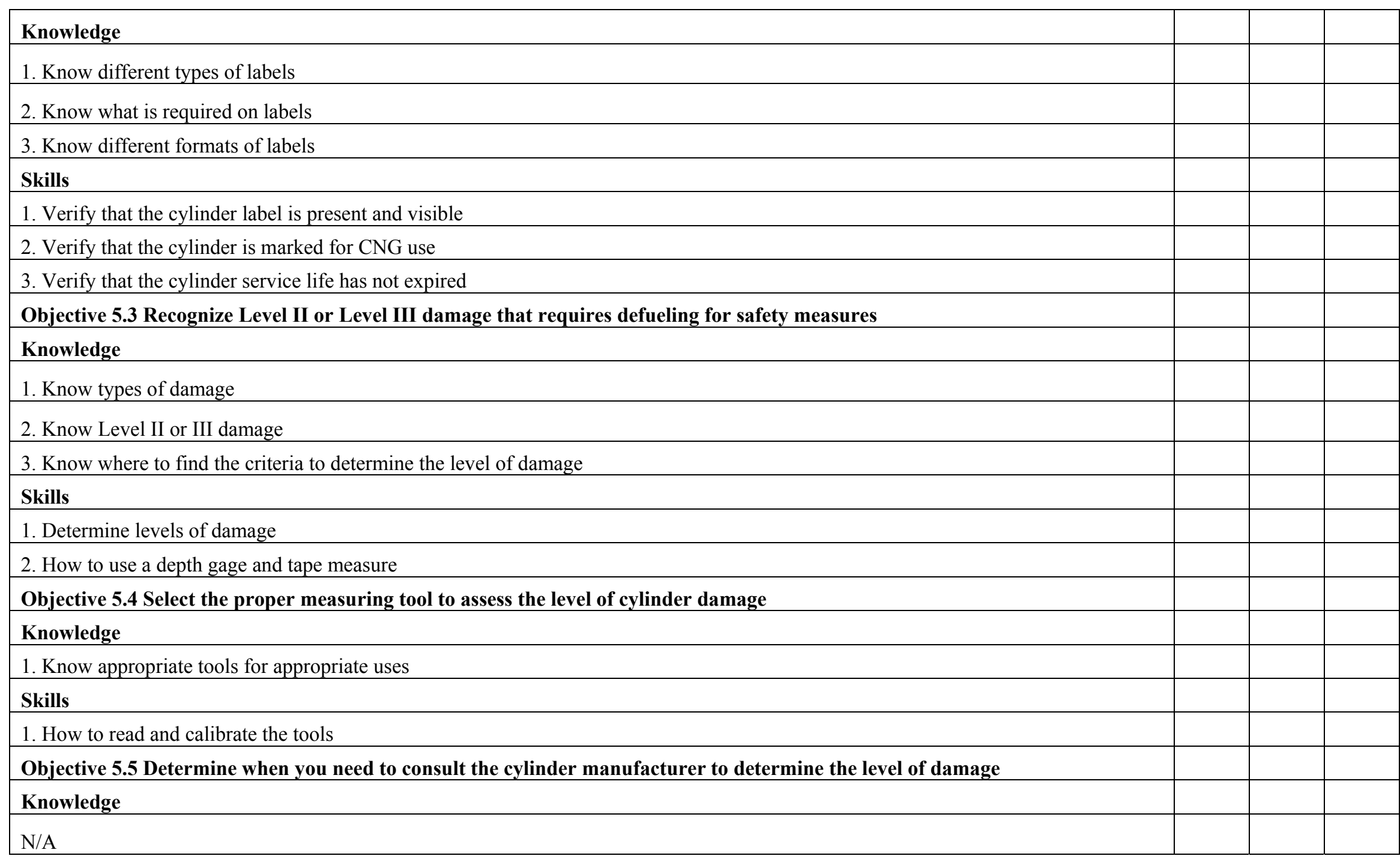




\section{Skills}

1. Applying standards to actual situations

Objective 5.6 Demonstrate familiarity with the concept of pressures and temperature relationships as applied to cylinder operating requirements

Knowledge

1. Know temperature/pressure relationship

2. Know the service pressure may not be the fill pressure

3. Know maximum permissible fill pressure Skills

1. How to read pressure gages and thermometers

Objective 5.7 Given a scenario, recognize the different types of CNG cylinder materials and construction Knowledge

1. Know different types of cylinder materials

2. Know construction methods

3. Know how different materials respond to damage mechanisms

\section{Skills}

1. Be able to recognize different cylinder materials and construction methods

\section{SECTION 6 ORIGINAL CNG FUEL SYSTEM INSTALLATION}

Objective 6.1 Determine that cylinders, brackets, components and shielding are installed to prevent damage and safety hazards

\section{Knowledge}

1. Know components applicable to NG systems

2. Know which components can be supported by piping

3. Know ground clearance requirements

4. Know acceptable locations for cylinders

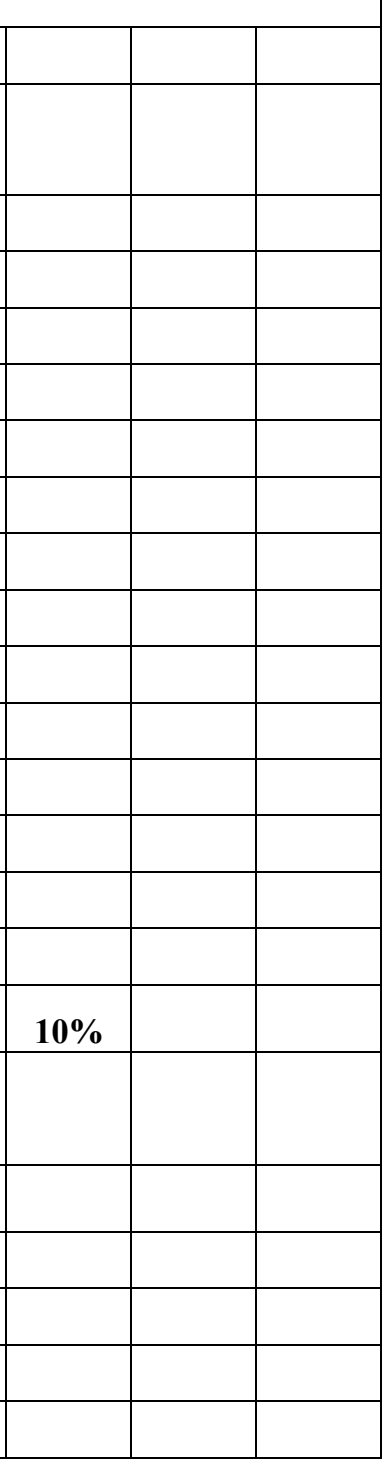




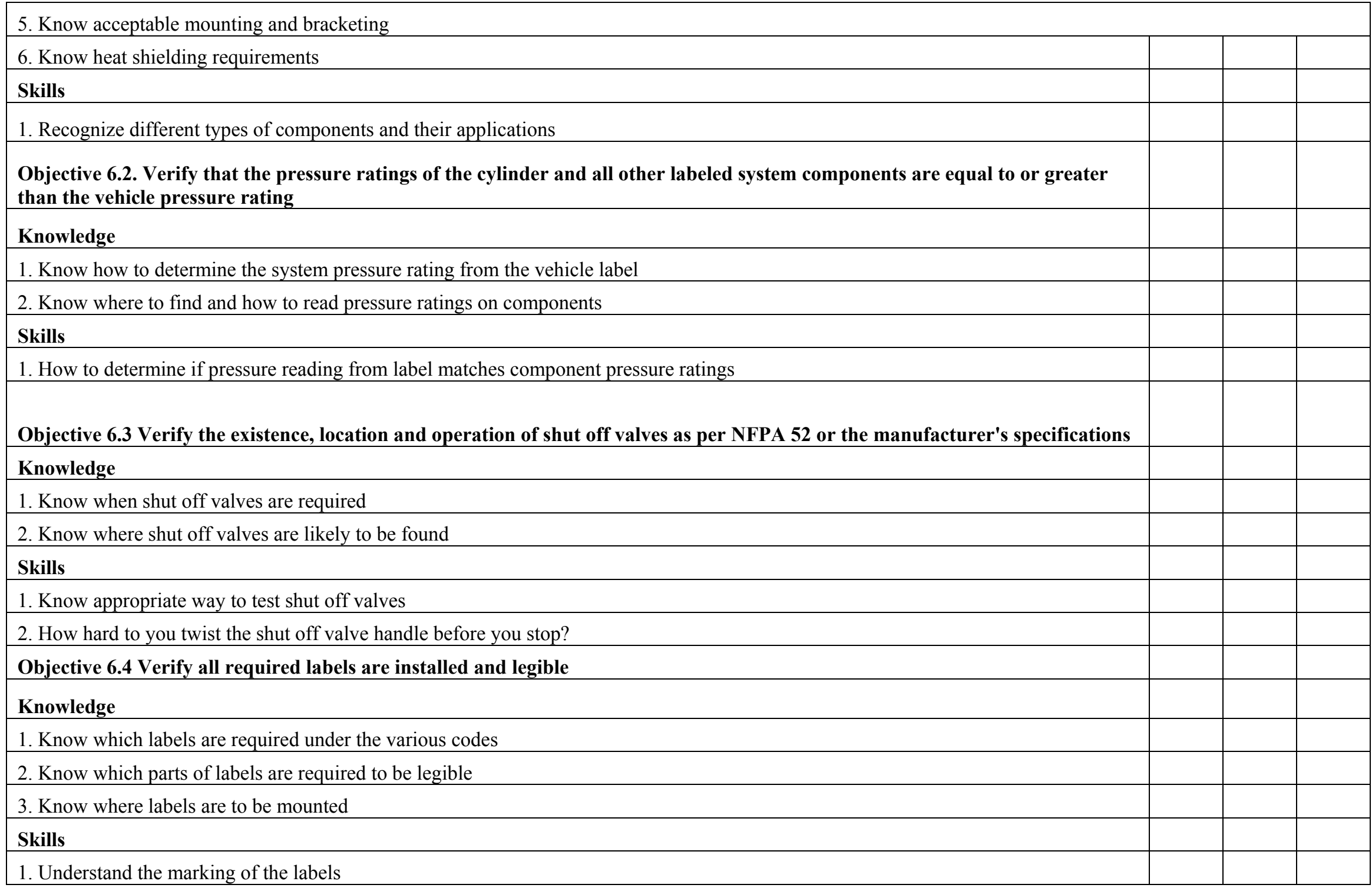




\section{SECTION 7 INSPECTION REPORTING}

Objective 7.1 Know how and when to fill out all areas of applicable inspection forms

Knowledge

1. Know which inspection form you need to use

2. Know to use the checklist during inspection

Skills

1. Operate a digital camera

2. Know how to write a comprehensive description and recommendation

\section{Objective 7.2 Given an inspection scenario, explain which actions you recommend}

Knowledge

1. Know the consequences of various defect levels

2. Know how to communicate to non-technical customers

3. Know sources for appropriate repair or replacement

Skills

1. Communication with public, vendors, manufacturers

\begin{tabular}{|l|l|l|}
\hline $\mathbf{5 \%}$ & & \\
\hline & & \\
\hline & & \\
\hline & & \\
\hline & & \\
\hline & & \\
\hline & & \\
\hline & & \\
\hline & & \\
\hline & & \\
\hline & & \\
\hline & & \\
\hline & & \\
\hline & & \\
\hline
\end{tabular}




\section{Table of Contents}

Section 1 Preparation for Inspection

Page 16

Section 2 Determine Inspection Requirements

Page 18

Section 3 Pressure Relief Device (PRD) Inspection

Page 23

Section 4 Physical Inspection of Brackets and High-Pressure Components

Page 25

Section 5 Physical Assessment of Cylinders

Page 27

Section 6 Original CNG Fuel System Installation

Page 35

Section 7 Inspection Reporting

Page 37

Appendix A Contact Information

Page 38

Appendix B Sample Checklists

Page 40

Appendix C Reference Resources

Page 44

Appendix D Test Taking/Sample Questions

Page 49 


\section{Section 1 Preparation for Inspection}

Natural gas is arguably, the safest, cleanest and most economical transportation fuel available today. While that is a rather bold statement, it can be backed up by the facts. As a CNG Fuel System Inspector, you will be part of this growing movement toward alternative fuels whether your goal is contributing to a cleaner environment, helping alleviate our dependence on foreign petroleum or reducing our national debt. In order for this industry to flourish it must maintain the enviable safety history it has to date. By joining the ranks of certified inspectors you can play a vital role in its development.

Before any CNG fuel system inspection begins, the inspector has quite a bit of work to do. He or she must gather as much information about the vehicle history, previous inspections, any accidents, etc., as is available. This can be done by interviewing the owner and/or operators, searching the existing service records, and looking for any obvious signs of collision damage from accidents or, more importantly vehicle fires.

Inspection forms will require information on the vehicle VIN number as well as any additional identifiers the owner or agency may use, e.g. license plate number. Now is the time to obtain and record these (see Appendix B).

Just as with a pre-flight check of an airplane, it's prudent to do a General Inspection of the fuel system and cylinder installation. A General Inspection is defined as an inspection for any signs of obvious or gross external damage, sounds or smells of possible natural gas leaks or any other potential problems that might require immediate attention. These inspections should also be included in any regular preventive maintenance programs or during any vehicle service or repair.
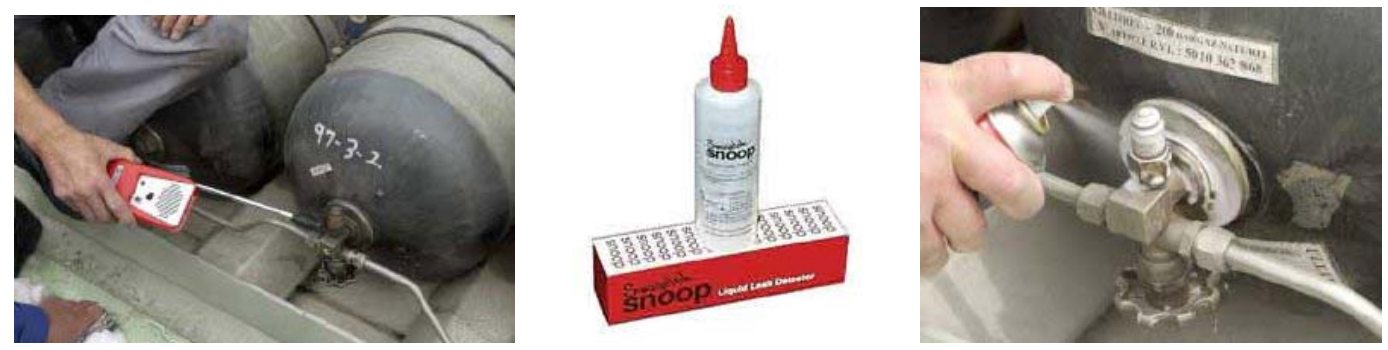

Figure 1.1 Leak Testing

In the rare event that there would be an urgent safety concern, the inspector should immediately decide on the appropriate action. If leaks are found or more serious levels of damage are suspected, stop and determine how to resolve the issue. This can be something as simple as turning off a cylinder or fuel line shut off valve to recommending CNG defueling and contacting emergency responders, before any further inspection is considered.

More commonly, the next step in preparing for an inspection is gaining access and cleaning all of the CNG fuel system components. This can be done either by the owner or designated staff personnel or by the inspector. Generally, time and money can be saved if the owner of the vehicle performs this operation. Regardless, some care needs to be exercised. Specifically, cylinder coatings and components may be susceptible to caustic cleaning solvents. Avoid soap solutions that contain ammonia as an example. Often questions regarding the use of high pressure washing equipment arise. Even if the cleaning solutions are appropriate care should be taken to avoid water intrusion into the PRD vent lines. The cylinder manufacturer should be consulted first, especially in the case of Type 4 composites. Regular sponges, wash rags, etc. are generally sufficient. 

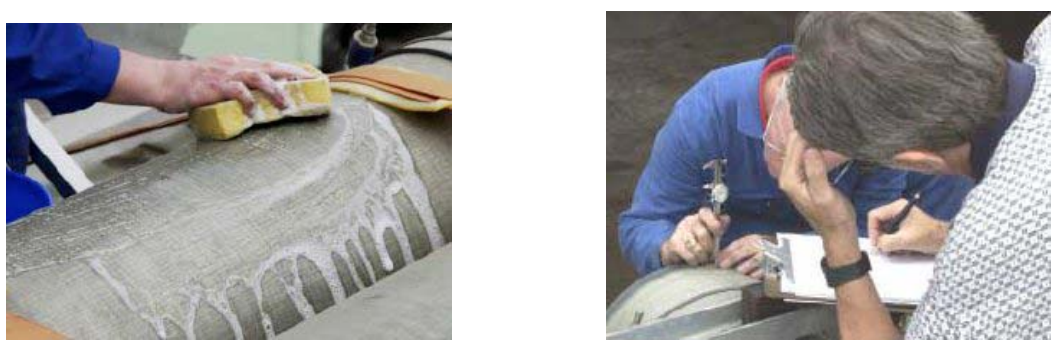

Figure 1.2 Cleaning and Recording

\section{Properties of Natural Gas}

Natural gas as provided by Local Distribution Companies (LDC's) or gas utilities has a distinctive odor. Generally, Ethyl Mercaptan is added to give a sulphur smell that allows detection at an early warning level, since pure methane, the major constituent of natural gas is odorless, colorless and tasteless. Any fuel has specific Upper and Lower Flammability Limits. In the case of natural gas, the lower limit is $5 \%$ by volume and the upper limit is $15 \%$ fuel to air. The Mercaptan concentration is designed to be detectable at $20 \%$ of the lower flammability limit or at a $1 \%$ level, fuel-to-air ratio, well short of the point of possible combustion.

It should be noted that Liquefied Natural Gas will not include the odorant and either on-board methane detectors will be added or a separate operation to reintroduce Mercaptan into the gas stream will be used.

Other properties that make this fuel safer are:

Natural gas is lighter than air. The specific gravity of natural gas is 0.6 compared to air at $1.0 \mathrm{SG}$. It will rise into the atmosphere if there is a leak and dissipate quickly.

By comparison, the flammability range for gasoline is $1 \%$ to $8 \%$, but remember that leaks from gasoline can accumulate and still be dangerous hours later, while natural gas will have dissipated.

To ignite natural gas, the ignition source must be at least $1,200^{\circ} \mathrm{F}$. The ignition temperature of gasoline is $540^{\circ}-800^{\circ} \mathrm{F}$, or about half of what is required for natural gas.

Below is a table of some of the important fuel characteristics:

\begin{tabular}{|c|c|c|}
\hline CHARACTERISTIC & GASOLINE & NATURAL GAS \\
\hline Chemical Symbol & $\mathrm{C}_{4} \mathrm{H}_{10}-\mathrm{C}_{12} \mathrm{H}_{26}$ & $\mathrm{CH}_{4}$ \\
\hline Vapor Density (Air=1.0) & 3.50 & $0.6-0.7$ \\
\hline Flammability Limits in Air & 1.4 to $7.6 \%$ & 5.0 to $15.0 \%$ \\
\hline 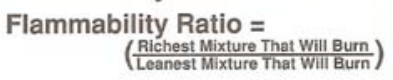 & $5.4: 1$ & $3: 1$ \\
\hline Boiling Point & $81^{\circ} \mathrm{F}$ to $437^{\circ} \mathrm{F}$ & $-260^{\circ} \mathrm{F}$ \\
\hline Air-Fuel Ratio & $14.7: 1$ & $16.5: 1$ \\
\hline Ignition Temperature & $540^{\circ} \mathrm{F}-800^{\circ} \mathrm{F}$ & $1,200^{\circ} \mathrm{F}$ \\
\hline Flame Speed (ft. per sec.) & 2.72 FPS & $2.20 \mathrm{FPS}$ \\
\hline Octane Rating & $86-93$ & $115+$ \\
\hline Fuel Quantity Measurement & Gallons & $\begin{array}{l}\text { Gasoline Gallon Equivalent } \\
\text { (GGE) (1 GGE = } 125 \mathrm{cu} \text {. ft.) }\end{array}$ \\
\hline Storage Pressure & Atmos. Pressure & $3,000-3,600 \mathrm{psi}$ \\
\hline Energy Content (BTU per lb.) & 18,400 & 20,500 \\
\hline
\end{tabular}

Table 1.1 (Courtesy General Motors) 


\section{Section 2.0 Determine Inspection Requirements}

The United States has led the world in NGV technology, particularly in the area of cylinder design and construction. From their beginnings in the space industry, lighter, composite cylinders have become the 'gold standard.' Several manufacturers have come and gone and as an inspector you should be familiar with them all. Appendix A contains a list of most of the major companies, past and present, that have been a part of that history.

There are four types of construction that are designated, appropriately, Type 1 thru Type 4.

\section{Types of CNG cylinders}

\begin{tabular}{|l|l|c|c|}
\hline Type & Description & $\begin{array}{c}\text { \% load contained } \\
\text { by metal }\end{array}$ & $\begin{array}{c}\text { \% load contained } \\
\text { by composite }\end{array}$ \\
\hline NGV2-1 (Type 1) & $\begin{array}{l}\text { All metal cylinders either steel or } \\
\text { aluminum }\end{array}$ & 100 & n/a \\
\hline NGV2-2 (Type 2) & $\begin{array}{l}\text { Cylinders with metal liner and a } \\
\text { hoop (center) wrapped composite }\end{array}$ & 55 & 45 \\
\hline NGV2-3 (Type 3) & $\begin{array}{l}\text { Cylinders with thin metal liner and } \\
\text { a fully wrapped composite }\end{array}$ & 20 & 80 \\
\hline NGV2-4 (Type 4) & $\begin{array}{l}\text { Cylinders with a plastic liner and a } \\
\text { fully wrapped composite }\end{array}$ & n/a & 100 \\
\hline
\end{tabular}

Beginning with Type 1, all steel or aluminum, each subsequent type reflects efforts to reduce weight by replacing metal with lighter weight composite (fiberglass or carbon fibers in a plastic resin) materials. The relative roles of metal and composites in the four designs can be understood by comparing the portion of the pressures retained by the liner and by the overwrap.

In Type 1 all of the internal force is contained by the metal. In Type 2 and 3 , the metal and composite share the pressures. The difference between the two is the coverage of the fiberglass overwrap. Type 2 covers the center of the cylinder only, while Type 3 wraps the entire cylinder. Type 3 will have a significant decrease in the metal liner thickness.

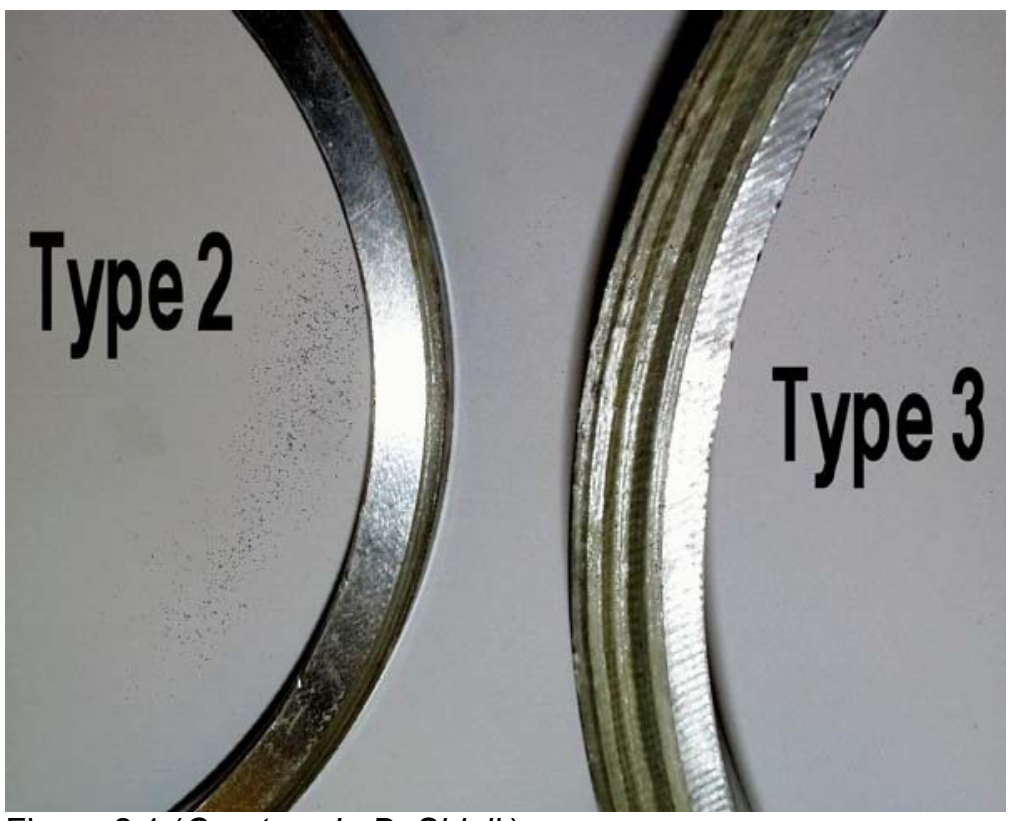

Figure 2.1 (Courtesy L. DaShiell:) 


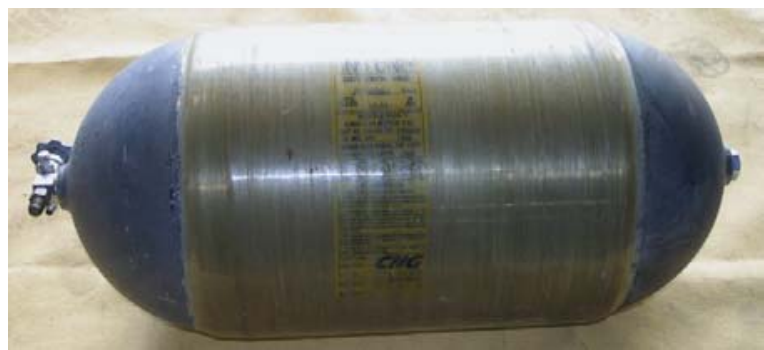

Figure 2.2 Type 2 Hoop Wrapped

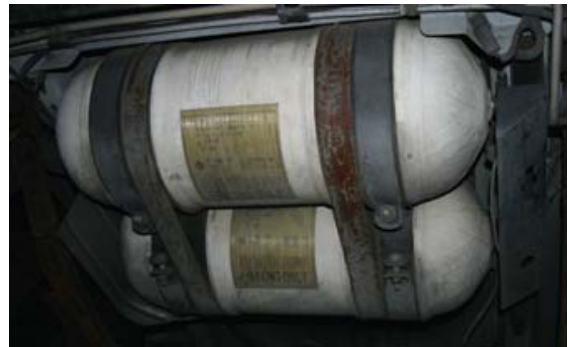

Figure 2.3 Type 3 Fully Wrapped

Type 4 is a full composite cylinder with no metal, except for the end boss for the valve. NOTE that without information from the manufacturer's label you can't easily tell the difference between a Type 3 and Type 4 cylinder.

Not only must inspectors be knowledgeable about the cylinder companies in the industry (see Appendix A), but they must be intimately familiar with the various standards those companies manufactured to or under. There is a priority to those standards as well.

The first and foremost resource for any inspection is always the manufacturers' guidelines. In the absence of specific manufacturer specification, the next two standards cover, in a generic overview, cylinder inspection and fuel system installation. Those are the Compressed Gas Association's (CGA) document C-6.4 Methods for External Visual Inspection of Natural Gas Vehicle (NGV) Fuel Containers and Their Installations and the National Fire Protection Association (NFPA) 52 Vehicular Fuel Systems Code.

Before any Detailed Inspection can begin, the inspector must identify which of these standards applies. There are currently two for CNG cylinders:

1. ANSI/CSA: NGV2 Compressed Natural Gas Vehicle Fuel Containers

2. DOT/NHTSA: FMVSS 304 Compressed Natural Gas Fuel Container Integrity (NOTE: this is a US government standard referenced in 49 CFR 571.304 Code of Federal Regulations)

These contain design qualification requirements that apply to manufacturers during production. They outline the allowable materials along with manufacturing and quality control tests. They are interesting to the inspector only for their rigor and as references.

NGV2 is a voluntary, industry driven standard that has been incorporated into the International ISO standard 11439. It details elaborate cycling, burst, impact, environmental, bonfire and rupture tests. FMVSS 304 is a US government (DOT - National highway Traffic Safety Administration) Federal Motor Vehicle Safety Standard and as such does have the force of law. They can be obtained on-line at: ANSI/CSA NGV2

http://webstore.ansi.org or www.csa-america.org

FMVSS 304 http://edocket.access.gpo.gov/cfr 2003/octqtr/pdf/49cfr571.304.pdf

They both specify periodic visual inspection of cylinders either every three years and/or 36,000 miles and after an accident or fire. They are the basis for the visual inspection protocols used in the United States today.

There were earlier standards, from the Compressed Gas Association, known as FRP-1 and FRP2. They were used by several cylinder manufacturers on a limited exemption basis by the Department of Transportation (see: 'DOT-E' in Fig. 2.4) before the current standards were developed. However, these cylinders are generally beyond their useful life (15 yrs. from the date [Mo-Yr] on the cylinder) and rarely seen. 
Cylinder labels can help determine the standards for a particular cylinder. Note the NGV2, DOT or DOT-E designations in the following examples:

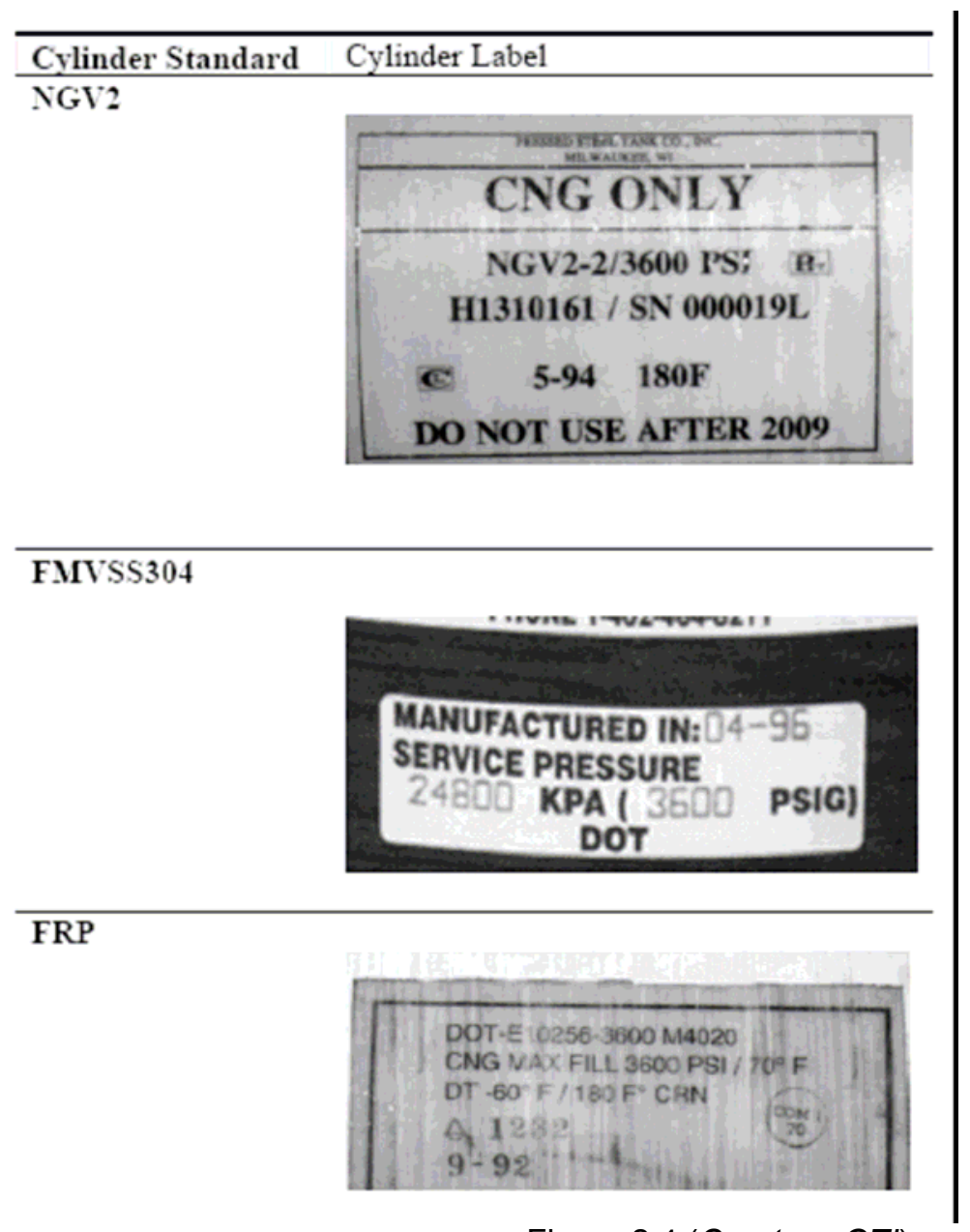

Figure 2.4 (Courtesy GTI)

Labels are also required to give critical information regarding the cylinder manufacturer, the service pressure, serial and/or model numbers and the container build date and expiration date.

CGA C-6.4 deals primarily with cylinder inspection whereas NFPA 52 deals with the rest of the fuel system installation. There are overlaps in both but this is generally the distinction between the two. As with any standard, they are dated and undergo periodic revisions. Each edition will generally have a history of the previous revisions or indication of the changes listed in the introductions. Inspectors should be aware of the changes and keep current on the standards.

This raises a common question when dealing with older vehicles. Which version should I use; the latest edition or the one that was in effect when the vehicle was produced? The best advice is to always follow the most current guidelines and if there are discrepancies use good judgment on whether they create a serious safety concern and finally, err on the side of caution. Where a system is built to an earlier standard and not required to be updated to a later version, it should not be faulted for not meeting the later standard unless there is an obvious safety issue. 
This issue comes up more with revisions to NFPA 52 and the installations of downstream components. Inspectors should be familiar with these as well. The major components of any NGV fuel system will consist of most, if not all, of the following:

- Fill receptacle

- One-way check valve(s)

- Fuel storage cylinder(s)

- Cylinder shut-off valve

- $\quad$ Pressure relief device (PRD)

- Manual ‘Quarter-Turn' shut-off valve
- Annealed stainless steel fuel tubing

- Shut-off valves (Lock-offs)

- Pressure regulator(s)

- Mixer assemblies (older vehicles)

- Gas injectors

\section{NATURAL GAS SYSTEM}
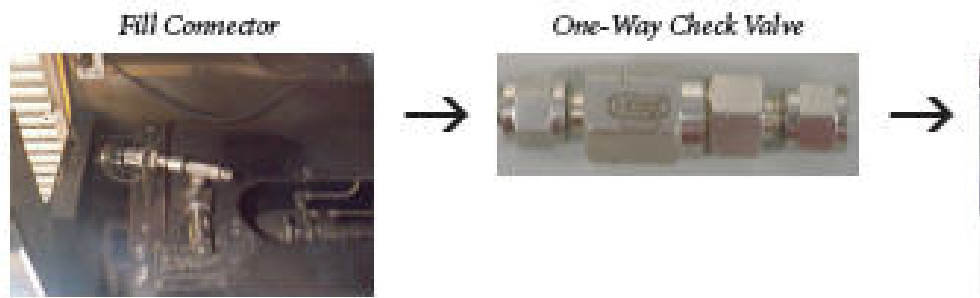

Fuel Storage Cylinder

Pressure Regulator
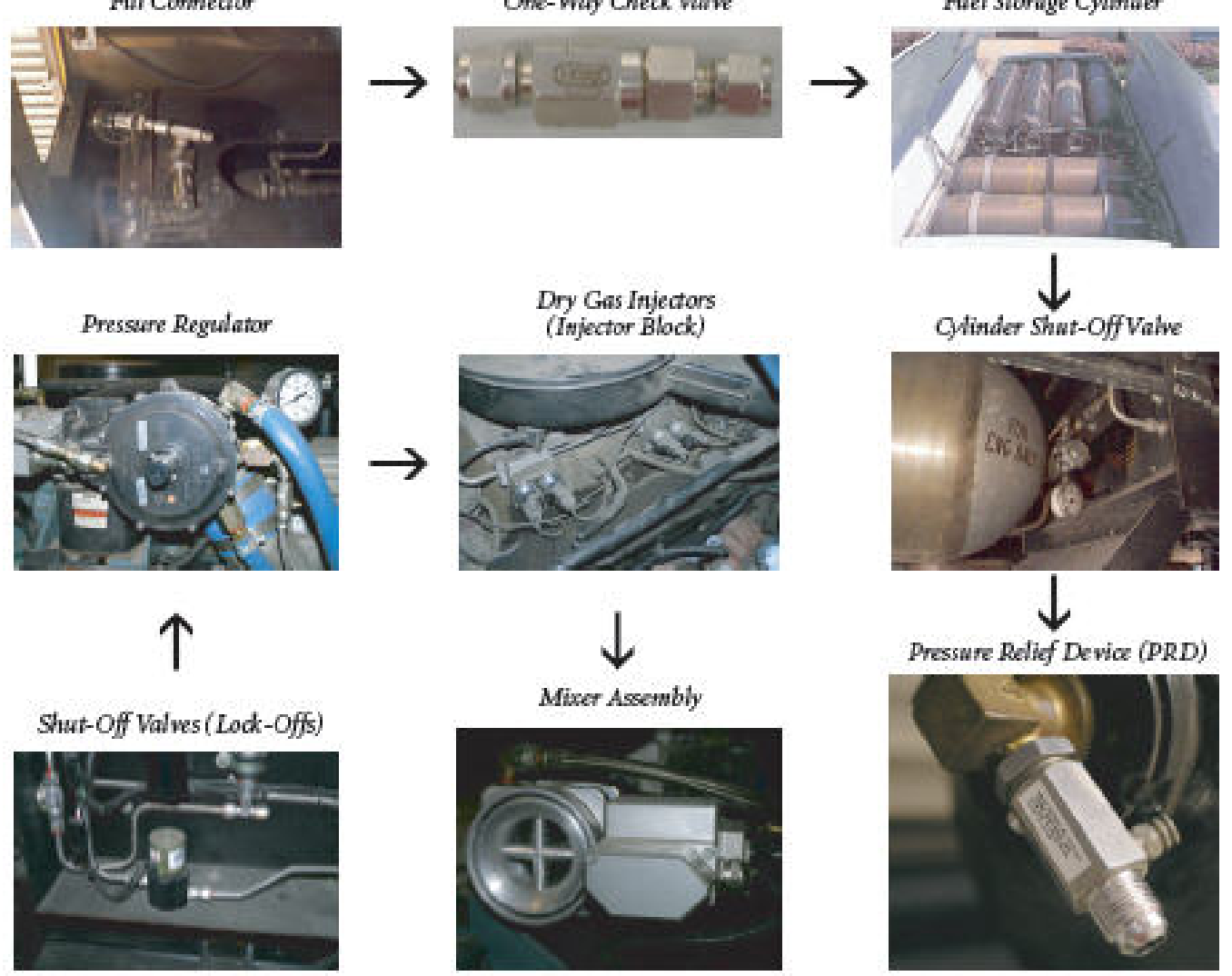

Cylinder Shut-Off Valve
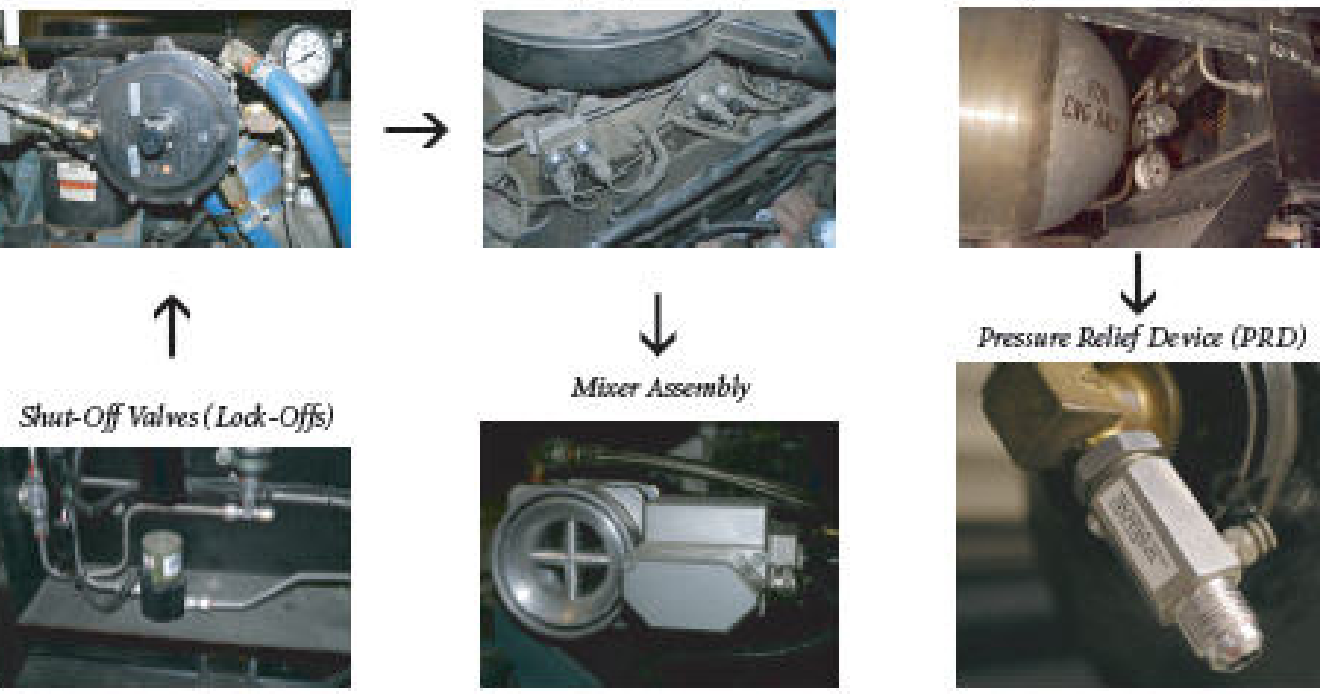

Pressure Relief Device (PRD)
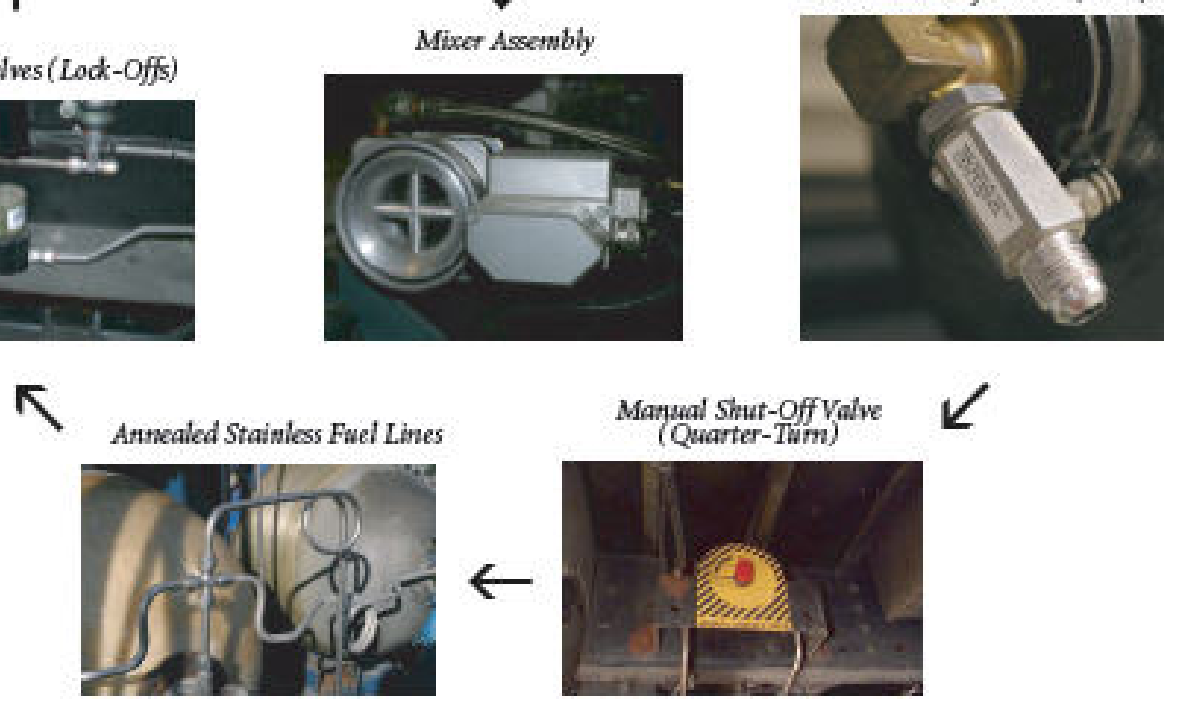

Figure 2.5 Typical Fuel System Components (Courtesy LBCC) 
In conclusion, these standards; the manufacturers' specifications, CGA C-6.4 and NFPA 52 (in that order) form the basis of the Detailed Visual Inspection protocol. A Detailed Visual Inspection, performed by trained, certified personnel, is the subject of this study guide.

The Clean Vehicle Education Foundation (CVEF) has made available a CD containing all of the manufacturers' specifications they were able to obtain. A copy can be obtained from CSA or CVEF's John Lapetz at jlapetz@cleanvehicle.org) 


\section{Section 3.0 Pressure Relief Device (PRD) Inspection}

Safety factors on NGV fuel systems are stringent. Cylinders are designed to withstand at least 2.25 times the working pressure. All of the downstream components are safe at three times their working pressures! (The battery of tests that cylinders have to pass in order to be certified can be found in the NGV2 standard) The device that assures cylinders will 'relieve' pressure in a fire is the Pressure Relief Device (PRD).

Pressure Relief Devices have a checkered history. Early versions had high failure rates and several were subject to recall. Today, new designs and improved manufacturing have all but eliminated these problems. The job of these devices is to relieve pressure from NGV cylinders in the event of a fire that could lead to a cylinder rupture. Standards do not require PRD's to vent during overpressurization of a cylinder (CNG fuel dispensers are relied on to control pressure), but they are required to vent during a vehicle fire.

There are many combinations of temperature and pressure mechanisms used in PRDs but two basic types are in wide use for compressed natural gas today.

\section{Thermally Activated}

This design is built to protect gas-containing vessels from rupture in case of fire. One style uses an alloy, called a eutectic, with a specific melting point, as an integral part of the PRD seal and a newer type that relies on the eutectic material only as a thermal triggering method.

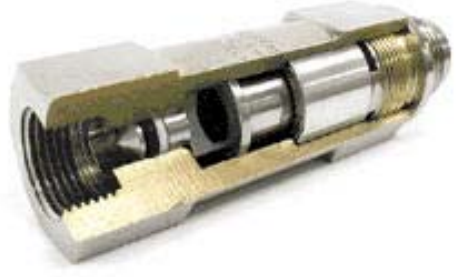

Figure 3.1 (Courtesy Circle Seal Controls)

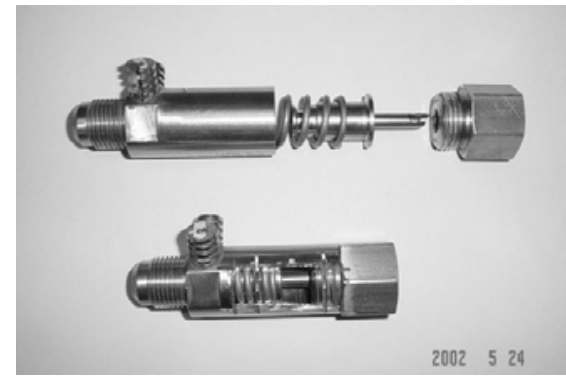

Figure 3.2 (Mirada- Courtesy L. DaShiell)

\section{Series Combination}

This type has a thin steel 'rupture' or 'burst disc' designed to burst at a predetermined pressure backed by a lead eutectic as a plug or thru a series of channels within a brass body. It requires excessive pressure and temperature to cause it to operate. It cannot prevent an improperly filled (overfilled) cylinder from rupturing due to hydrostatic pressure at room temperature, or any temperature below the melting point of the fusible material. Both the burst pressure and temperature are generally stamped on the PRD body.

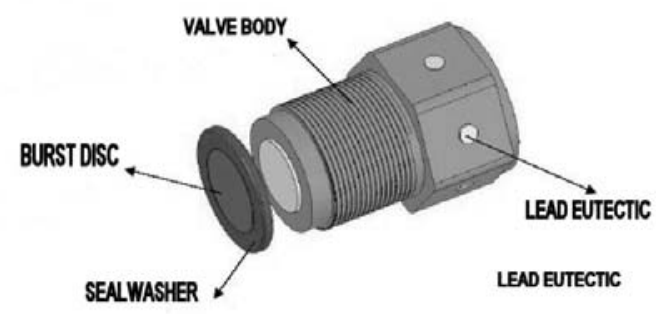

Figure 3.3 Series PRD 
There are two conditions, aside from mechanical damage, with PRD's that an inspector must look for, namely leakage and premature extrusion of the eutectic. The first requires some form of leak testing and in the case of attached vent lines may present some challenges to access or assure that the PRD is OK.

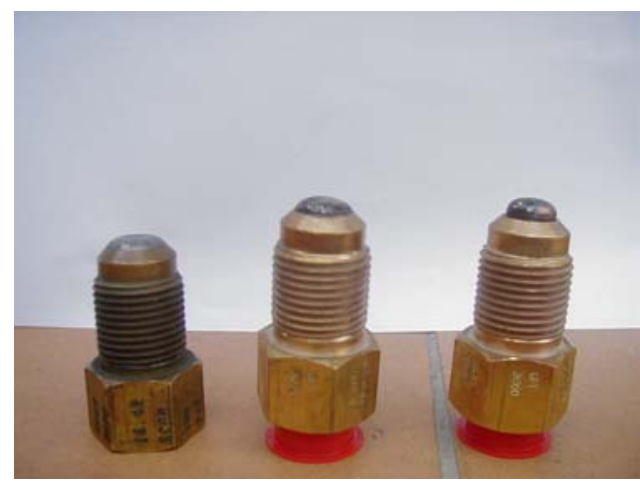

By the same token, evaluating an extruded eutectic can be difficult. Consider these three Pressure Relief Devices. It would appear that the lead plug on the right has begun to extrude but the center and right PRD's are both new! So the difference between a manufacturing anomaly and an actual extruded eutectic might not be obvious. You must see evidence of damage or leaking of the fusible material before condemning it.

Figure 3.4 PRD

Mechanical damage can occur when moisture is allowed to collect at the PRD. During freezing weather cases have been reported where the PRD released even though there was no indication of fire. NFPA 52 requires the venting system prevent water, dirt, or any foreign objects from collecting in the vent lines or PRD (see Section 6.4 NFPA 52, 2006).

This is the biggest cause of PRD failure, and many designs overlook this. Check for evidence of water intrusion:

- Loose or stretched PRD's

- Loose fittings on the outlet side

- Leaks

- Water marks (soap scum, lime, etc.) in the vent tube or the PRD

- Evidence of reverse pressure on the PRD (more pressure in the outlet than in the tank) [Ice can generate over 10,000 psi]

NOTE: Rubber caps that have been knocked off, particularly by cleaning brushes or tree branches can cause this.

- Rubber caps that break down in UV.

- Lack of caps or drain holes

- Vent tubes that run straight up. They should have a bend so that any water that does accumulate doesn't fill the PRD.

- Caps that are to tight that allow the inevitable permeation of gas through the PRD causes a pressure build-up that blows off the cap. Caps should have the ability to vent the tiny flow may come through the PRD. 


\section{Section 4.0 Inspection of Brackets and High-Pressure Components}

Inspection of cylinder mounting assemblies is the second largest portion of the certification exam. This section, along with Section 6 dealing with the rest of the system components, comprises $28 \%$ of the questions.

NFPA 52 is the primary resource for requirements on the installation of NGV Fuel Systems. The current edition (2006) devotes the entire Chapter 6 to Installation of cylinders, venting systems, piping and valves, pressure gauges fill receptacles and regulators. The inspector should be familiar with all of them. What follows is a summary (not intended to be complete) of the major points.

Beginning with $\underline{\text { cylinders, }}$

- May be located within, below or above the passenger compartment

- No portion can be located ahead of the front axle or behind the point of attachment of the rear bumper

- Must have the label visible

- Cannot be within eight inches of the exhaust system without proper shielding

- Must be mounted at least nine inches above the ground for vehicles over 127" wheel base or at least seven inches for vehicles with 127" or less measured with the tires deflated.

- Be capable of restraining the cylinder when subjected to a force of eight times the weight of the cylinder in six principle directions without moving over one half inch

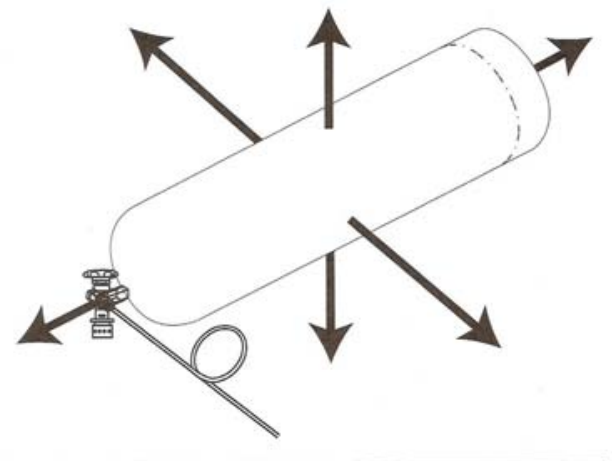

Figure 4.1 Six principle directions

- Incorporate manufacturer's recommended brackets with rubber gaskets and torque to specification

- Be properly shielded from sunlight, cargo or road debris

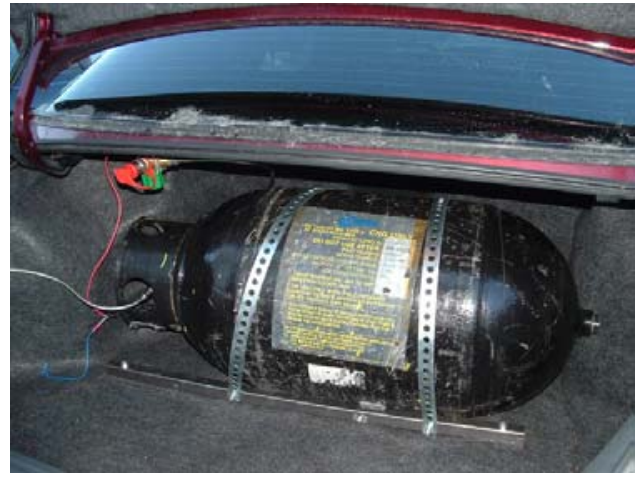

Figure 4.2a Bad Mounting

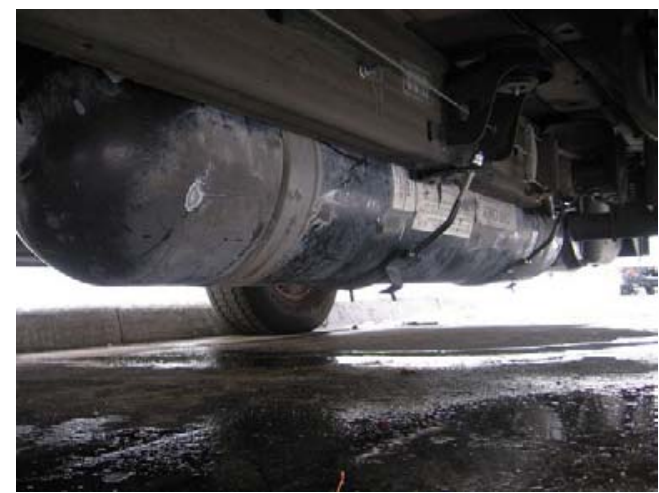

Figure 4.2b No Shielding 
- Cylinder valves and fittings mounted in the passenger compartment must be properly vented to the outside using tubing or a vapor barrier

- Rubber gaskets shall be installed under the clamping bands to provide insulation between the bands and the containers

- Must not adversely affect the driving characteristics of the vehicle

Venting systems included here can refer either to high pressure PRD vent lines or low pressure 'vent bags' for the neck of the cylinder and all fittings within the passenger compartment. (The trunk is considered part of the passenger compartment.)

- All potential leak points must be protected (cylinder valves and fittings)

- High pressure PRD vent line must be metallic, and electrically conductive

- Cannot vent into a wheel well

- Must prevent water, dirt or other contaminants from collecting in the lines or PRD

- The PRD and cylinder must be in the same vehicle compartment

- Low pressure enclosures must be gastight, made of low-density polyethylene or equivalent, free of tears.

- Vent lines must be adequately secured, have a burst pressure at least $1 \frac{1 / 2}{2}$ times the pressure of an activated PRD and capable of withstanding $1120^{\circ} \mathrm{F}$ for 20 minutes

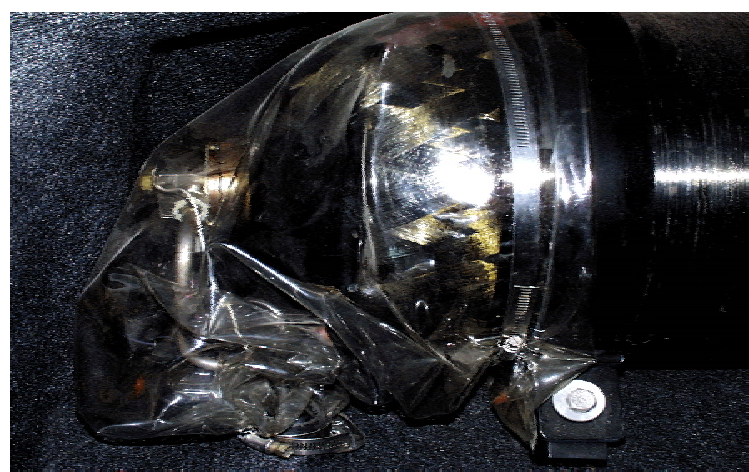

Figure 4.3 Polyethylene vent bag (L. DaShiell)

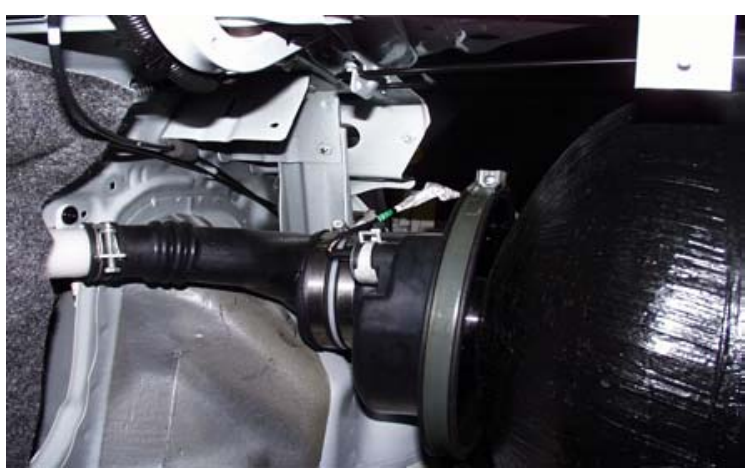

Figure 4.4 Formed vent cover (L. DaShiell)
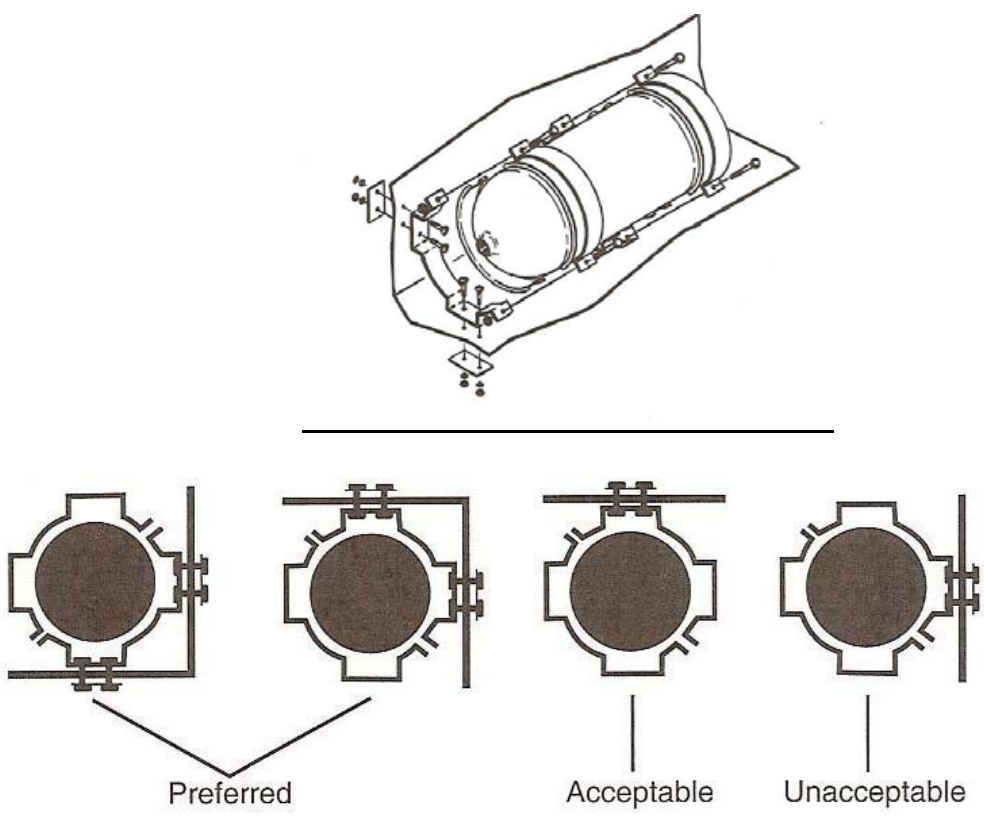

Flgure 4.5 Proper and improper cylinder mounting procedures (Courtesy CNG Cylinder Company) 


\section{Section 5.0 Physical Assessment of Cylinders}

This section provides the inspector with a description of some types of damage that can occur with CNG cylinders. As it is not possible to address every possible damage scenario, these are the most common. These guidelines are defined in specific detail in the Compressed Gas Associations' C-6.4 document. This is also the largest portion of the exam $-38 \%$.

The cylinder manufacturer's recommendations (if available) always take precedence and are the primary source for information.

CGA C-6.4 lists general guidelines. CNG cylinder damage is classified in three levels. The levels are as follows:

Level 1 - any scratch, gouge, or abrasion with a damage depth of less than or equal to .010 inch. Level 1 damage is acceptable and does not need to be repaired. * Refer to CGA C-6.4 as a guideline for each type of damage and the allowable limits. Some manufacturers allow different limits over .010 inch for newer tanks. Always consult the manufacturer of the cylinder if damage exceeds .010 inch for their exact requirements.

Level 2 - any scratch, gouge, or abrasion with a damage depth of .011 to .050 inch.

Level 2 damage requires rework (either in the field or by the manufacturer), a more thorough evaluation, or destruction of the cylinder depending on severity.

Level 3 - any scratch, gouge, or abrasion with a damage depth greater than .050 inch. Level 3 damage is severe enough that the cylinder cannot be repaired and must be destroyed. All fire, and chemical damage is Level 3 , if it does not wash off.

Level 1 cut or abrasion damage is generally .010 inch or less according to CGA C-6.4. However, the manufacturer is the final authority having jurisdiction over damage levels. Some Level 2 damage may be repaired in the field depending upon manufacturer's guidelines and procedures. Between Level 2 and Level 3 , there are acceptable field repairs available to resolve some conditions to a level where they can be resolved to Level 1 and returned to service. There is also Level 2 damage criteria where the manufacturer has to complete the repair, but the cylinder can be re-certified and returned to service. Depending upon the type of cylinder and the manufacturer, the point at which damage becomes Level 3 varies. Some, like Dynetek Type 3 cylinders, allow rework by the manufacturer for cuts from .030 to .050 inch damage. Some Type 4 cylinders, such as the Tuffshell by Lincoln Composites, allow rework by the factory for scratch, gouge, or abrasion damage from .036 to .050 inch and condemn the cylinder after .050 inch. The area where the damage occurs can alter the allowances, e.g., the radius of the dome. When in doubt, check the manufacturer's specific tolerances.

*NOTE: Although Level 1 damage does not require rework, all damage must be recorded. 


\section{Damage Types}

\section{Surface Corrosion or Pitting}

This is most prominent on Type 1 and 2 cylinders due to exposed metals. Many newer cylinders have epoxy painted coatings to help prevent this damage. Corrosion should be cleaned off, evaluated, and resealed to prevent further damage. Corrosion or pitting over .030 inch in depth can be Level 3 damage if it covers considerable surface areas of the cylinder (see $\$ 7.6 .1 .4$ of CGA C-6.4). Type 1 cylinders must be evaluated for loss of wall thickness. This will require specifics on the original cylinder. CGA C-6 provides additional guidance and lists wall thicknesses for certain steel cylinders.
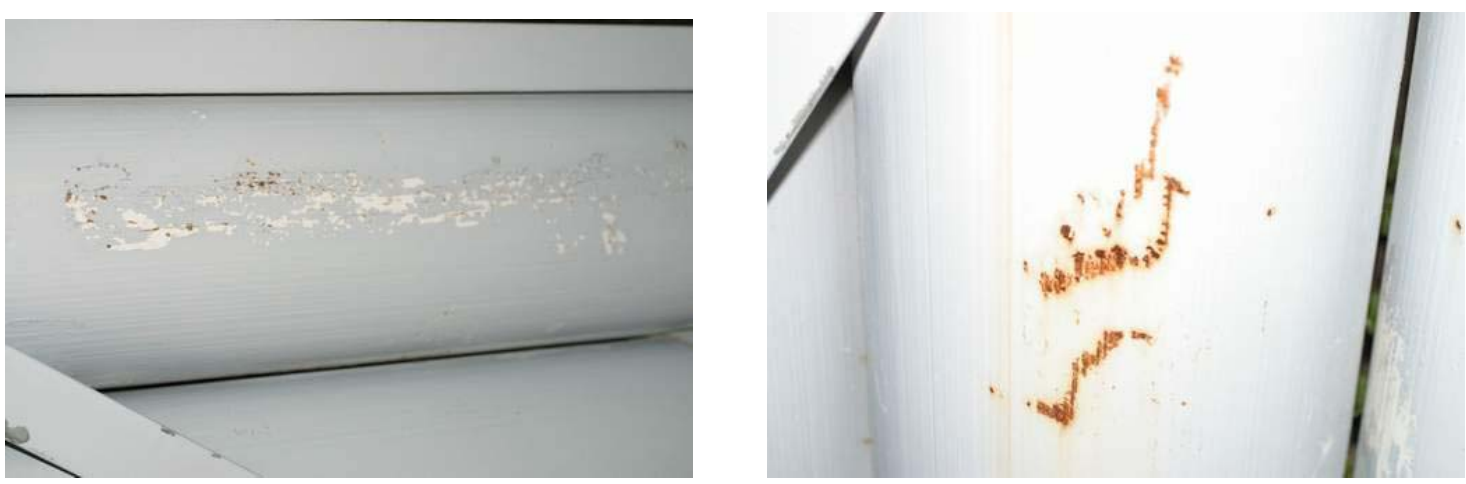

Figure 5.1 Corrosion

\section{Fatigue or Stress Corrosion Cracking}

This occurs when the cylinder is cycled repeatedly causing expansion and contraction of the cylinder, which is usually a sign of age or over pressurization. The fiber wrap cracks longitudinally causing loss of the cylinder pressure safety factor. Any identified stress or fatigue cracking is Level 3 damage. A contributing factor can be chemical attack e.g., battery acid.

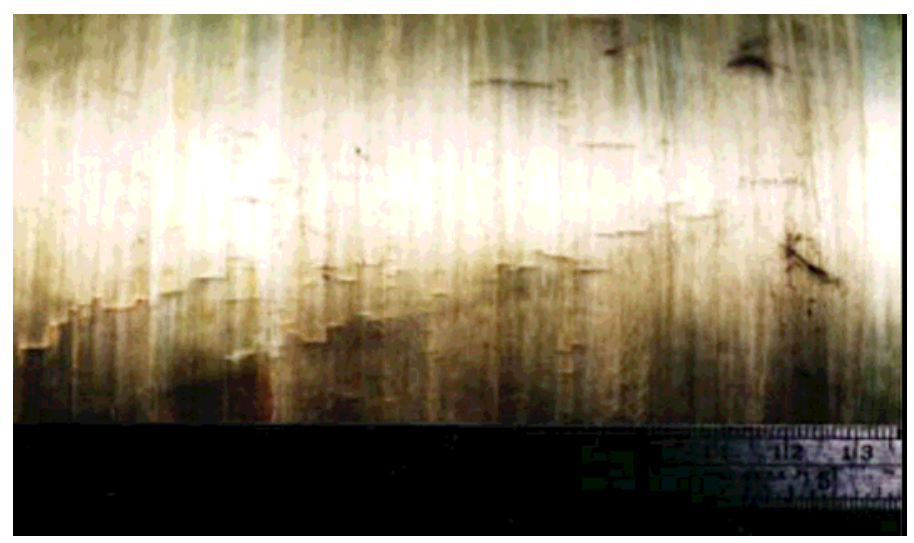

Figure 5.2 Stress Corrosion Cracking (SCC)

\section{Scuffing or Abrasion Damage}

This is very common on cylinders that are mounted underneath the vehicle, if shielding is inadequate or the cylinder is exposed. Type 2, 3, and 4 cylinders with less than 0.010 in have Level 1 damage. 


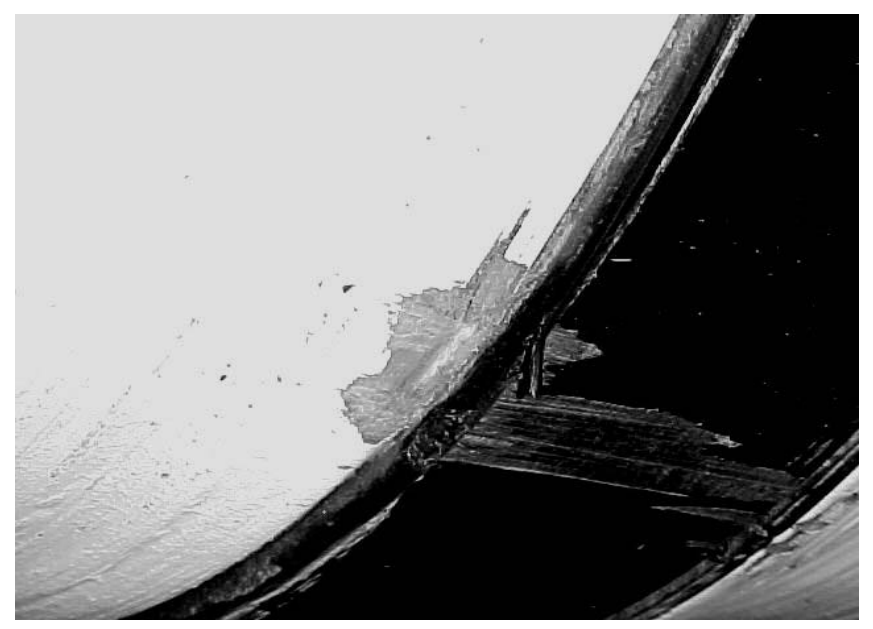

Figure 5.3 Abrasion Damage

\section{Surface Cuts and Scratches}

These are caused by a foreign object coming in contact with the cylinder. Brackets and other items rubbing against the cylinder can also cause this damage. Cuts or gouges less than $0.010 \mathrm{in}$. deep are defined as Level 1 damage regardless of length, number or direction. Cuts or scratches greater than or equal to 0.010 in deep are defined as Level 2 or Level 3 damage and may require the use of manufacturer's guidelines (see $§ 7.5 .2$ of C-6.4).

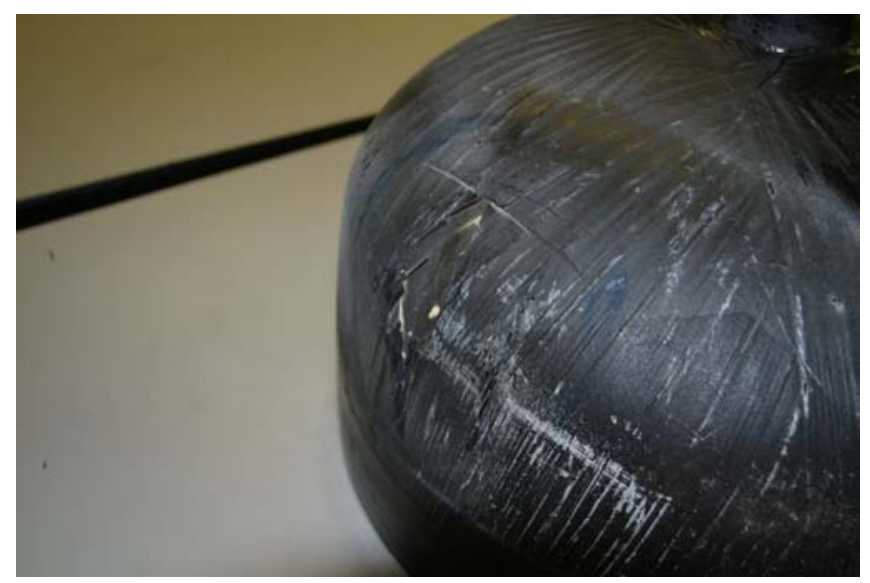

Figure 5.4 Cuts

\section{Blunt or Sharp Object Impact Damage}

Minor dents up to $1 / 16$ " inch and greater than 2 in. diameter on Type 1 cylinders can be tolerated. However, blunt impact damage on Type 2, 3, and 4 cylinders is very hard to evaluate and can be dangerous, especially on Type 4 cylinders. Extreme care must be taken to determine if any deformation of the cylinder is present indicting fiber damage. This is a sign that the cylinder may not be structurally sound and is considered Level 2 or 3 damage. 


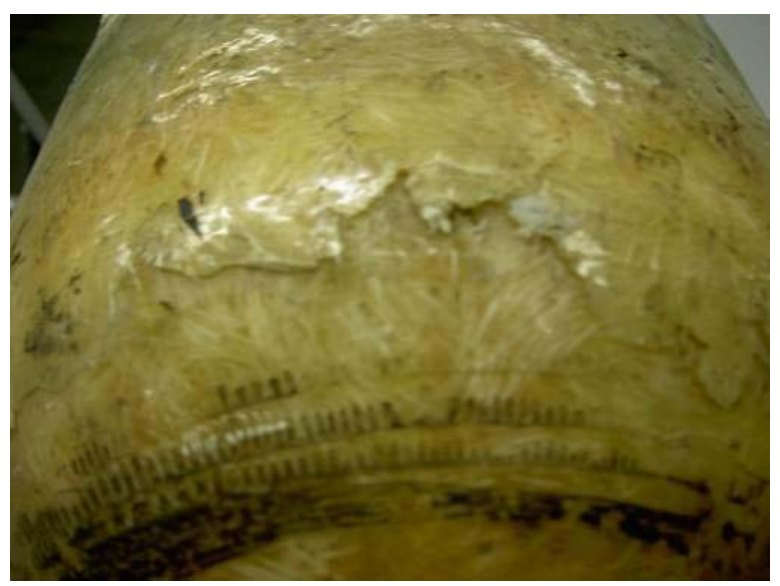

Figure 5.5 Impact damage

\section{Collision, Fire or Heat Damage}

Any indication that the vehicle has been involved in an accident or fire requires careful examination of the cylinders. Follow the manufacturer's guidelines for such damage. Generally, if Type 2, 3, or 4 cylinders are exposed to excessive heat, or any discoloration occurs that does not wash off, it is considered Level 3 damage.

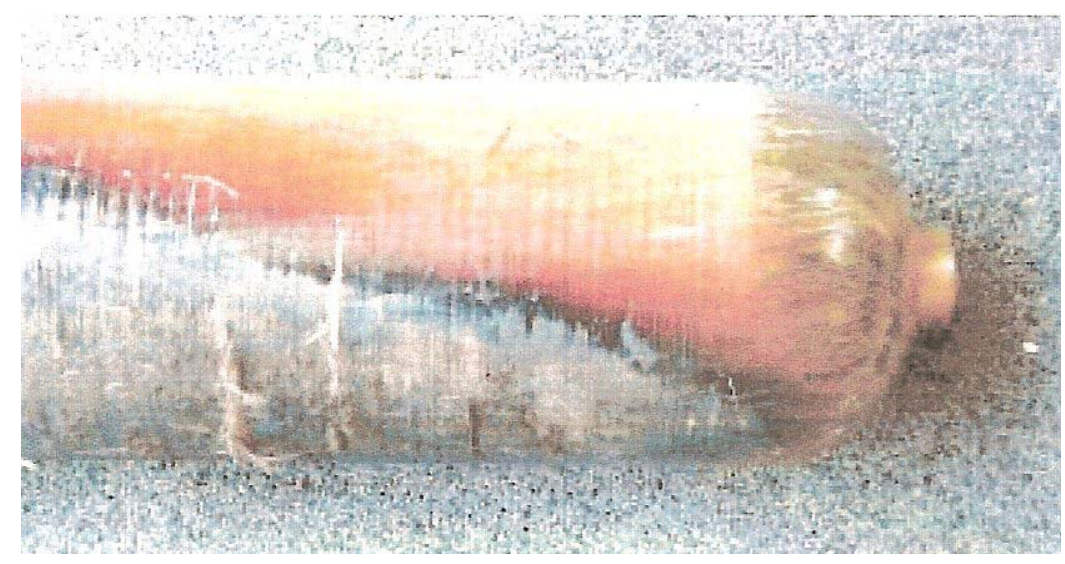

Figure 5.6 Heat damage

\section{Chemical Attack}

Acids and other chemicals can severely damage the cylinder wrap and possibly the metal itself. Extreme care should be taken to identify and neutralize any chemicals spilled on the cylinder. Only minor discoloration is allowed after neutralization and a very careful inspection should be performed to make sure the chemical did not get between the wrap and the cylinder where unseen damage could progress. Chemical attack can also lead to Stress Corrosion Cracking (see No.2 above). Comdyne cylinders suspected of being exposed to acid should be depressurized as soon as possible to prevent rupture. The cylinders should then be removed from service, rendered unusable, and disposed of. Any other CNG cylinder that has been exposed to acid should be examined in accordance with either the vehicle or cylinder manufacturer's recommendations. Ref. CVEF Safety Advisory. 


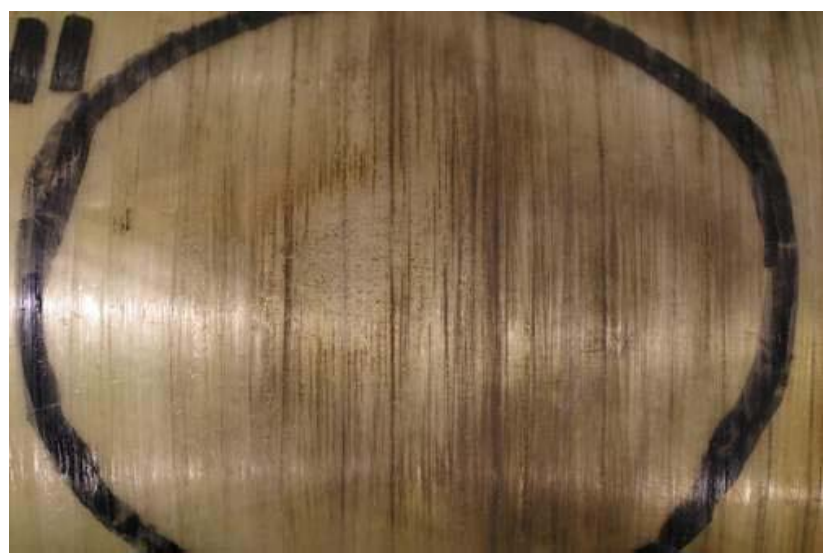

Figure 5.7 Chemical damage

\section{Bulging, Bowing of Cylinder Wall}

All visible outward bulges indicate a problem with the cylinder material and should be considered Level 3 damage (\$7.6.2 CGA C-6.4). This is most prominent on Type 1 and the exposed surfaces of Type 2 cylinders.

\section{Weathering/UV Damage}

Ultraviolet light can cause damage to the wrap which must be addressed. In most cases the manufacturers have coatings on the cylinder to prevent this damage. Excessive weathering results in Level 3 damage, as the fibers are damaged.

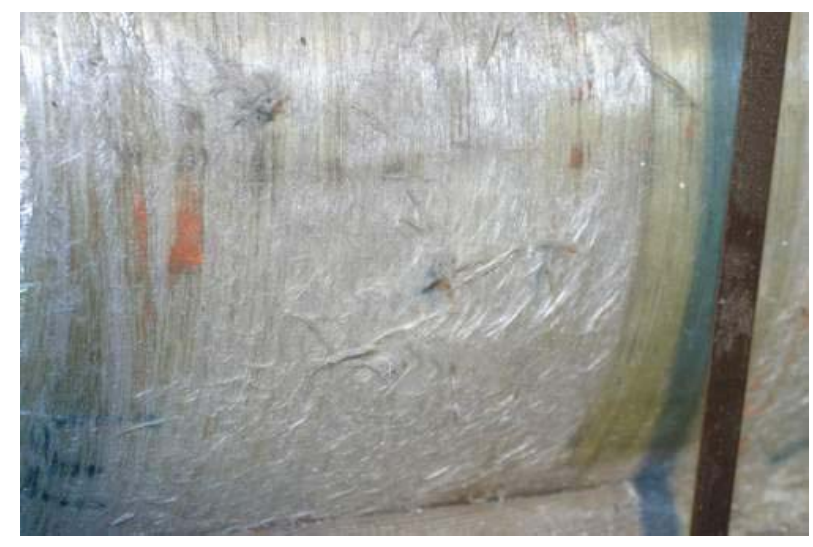

Figure 5.8 UV damage

\section{Over-Pressurization, Leaks}

Any cylinder that leaks or has been exposed to over 1.25 times its service pressure is to be considered to have Level 3 damage. Bubbles on the surface of Type 4 cylinders may be indications of leaking of the liner and could be Level 3 damage. Further tests to determine if it is trapped air between the liner and the overwrap or is, in fact, leaking gas should be performed. 


\section{Labeling}

A label that cannot be seen requires repair and, if it is missing or illegible it becomes Level 3 damage.

It should be noted that in addition to the cylinder label OEM manufacturers or after-market conversions must also have a label (usually located in the engine compartment) that identifies the vehicle as being CNG-fueled and includes; service pressure, the installers name or company, the cylinder retest or expiration date and the total container(s) water volume in gallons or liters.

According to NFPA 52, another label is required at the fill connection receptacle that specifies CNG, the system working pressure and the cylinder retest or expiration date. Most OEM's also follow this recommendation.

\section{All the following are to be considered Level 3 damage:}

- A missing cylinder label

- All fire damage, if it leaves discoloration

- All chemical damage, if it leaves discoloration

- Any noticeable discoloration that cannot be washed off

- Stress corrosion cracking

- Impact damage on Type 4 cylinders 
The following table reprinted from CGA-6.4 by permission gives a detailed breakdown of generic cylinder damage limits.

\begin{tabular}{|c|c|c|c|c|}
\hline Condition & Level 1 & Level 2 & Level 3 & $\begin{array}{l}\text { C-6.4 } \\
\text { ref. }\end{array}$ \\
\hline \multicolumn{5}{|c|}{ Composite and metal containers (All container types) } \\
\hline Labeling & $\begin{array}{l}\text { Legible and clean. Required } \\
\text { information all legible. } \\
\text { Information correlates with } \\
\text { vehicle service }\end{array}$ & $\begin{array}{l}\text { Only manufacturer and } \\
\text { serial number is clear. } \\
\text { Other required information } \\
\text { is illegible }{ }^{1}\end{array}$ & $\begin{array}{l}\text { Serial number illegible or } \\
\text { untraceable, or unidentified } \\
\text { manufacturer or model/part } \\
\text { number. Beyond service } \\
\text { life. }\end{array}$ & 7.9 \\
\hline Cuts/scratches/gouges & $<0.01 \mathrm{in}(.025 \mathrm{~mm})$ & 2 & $\geq 0.01(0.25 \mathrm{~mm})$ deep $^{2}$ & 7.5 .2 \\
\hline Charring/sooting & None & Washes off $^{2}$ & Permanent marking & 7.5 .3 \\
\hline Gas leakage & None & Bubble test negative & Bubble test confirms leak & 7.5 .4 \\
\hline Chemical attack & $\begin{array}{l}\text { Cleans off. No residue or } \\
\text { affect. Chemical is know not } \\
\text { to affect container type }\end{array}$ & $\begin{array}{l}\text { Discoloration is minor after } \\
\text { cleaning, no material loss }\end{array}$ & $\begin{array}{l}\text { Permanent discoloration, } \\
\text { loss/disruption of material }^{1}\end{array}$ & 7.5 .5 \\
\hline Weathering (UV effects) & None & $\begin{array}{l}\text { Coating disruption and/or } \\
\text { loss }^{2}\end{array}$ & $\begin{array}{l}\text { Excessive. Look for other } \\
\text { condition effects }\end{array}$ & 7.5 .6 \\
\hline $\begin{array}{l}\text { Involved in a collision, } \\
\text { accident or fire. } \\
\text { Container subjected to } \\
\text { high or unknown heat. }\end{array}$ & $\begin{array}{l}\text { No indications and vehicle } \\
\text { owner know of no accident, } \\
\text { fire or heat exposure }\end{array}$ & $\begin{array}{l}\text { Owner reports vehicle was } \\
\text { in a fire, accident or } \\
\text { exposed to heat }{ }^{1}\end{array}$ & $\begin{array}{l}\text { Indications of vehicle an/or } \\
\text { container impact or heat } \\
\text { damage }^{1}\end{array}$ & $\begin{array}{l}7 . \\
7.2 \\
7.5 .3 \\
\end{array}$ \\
\hline Overpressurization & $\begin{array}{l}\text { No indication or knowledge } \\
\text { by inspector }\end{array}$ & & $\begin{array}{l}\text { Cylinder reported to have } \\
\text { been pressurized above the } \\
\text { applicable design standard }\end{array}$ & 7.5 \\
\hline \multicolumn{5}{|c|}{ Composite containers (Type 2,3 and 4 ) } \\
\hline Impact & None & $\begin{array}{l}\text { Dents, fiber breaking, cuts, } \\
\text { etc. }{ }^{1}\end{array}$ & $\begin{array}{l}\text { Permanent deformation of } \\
\text { container }^{1}\end{array}$ & 7.7.2 \\
\hline Stress corrosion cracking & None & Questionable or unsure $^{1}$ & Any identified SCC & 7.5 \\
\hline Abrasion & $<0.010$ in $(0.25 \mathrm{~mm})$ deep & 2,3 & $\geq 0.01 \mathrm{in}(0.25 \mathrm{~mm})$ deep & 7.7 .1 \\
\hline \multicolumn{5}{|c|}{ Metal containers (Type 1 and the exposed metallic portions of Type 2) } \\
\hline Bulging/bowing & $\begin{array}{l}\text { None or shape can be } \\
\text { identified as 'banana' or } \\
\text { bower (Type } 1 \text { containers } \\
\text { only) }\end{array}$ & $\begin{array}{l}\text { All visible bulges are Level } \\
3 \text { damage. See }{ }^{1} \text { for } \\
\text { questionable bow }\end{array}$ & $\begin{array}{l}\text { Visible bulge or bowed } \\
\text { shape interferes with proper } \\
\text { mounting }\end{array}$ & 7.6 .2 \\
\hline Corrosion, pits & $<0.035$ in $(0.889 \mathrm{~mm})$ deep & 1 & $\geq 0.035$ in $(0.889 \mathrm{~mm})$ deep & 7.6.1.1 \\
\hline Corrosion, line pits & $<0.03$ in $(0.76 \mathrm{~mm})$ deep & 1 & $\begin{array}{l}\geq 0.03 \text { in }(0.76 \mathrm{~mm}) \text { deep. } \\
\text { Any depth over } 6 \text { in long } \\
(15.2 \mathrm{~cm})\end{array}$ & 7.6.1.4 \\
\hline Dents & None & Minor dent(s) ${ }^{1}$ & $\begin{array}{l}\leq \text { in }(51 \mathrm{~mm}) \text { diameter or } \\
\geq 0.0625 \mathrm{in}(1.588 \mathrm{~mm}) \text { deep }\end{array}$ & 7.6 .3 \\
\hline Abrasions & None & Minor & $\begin{array}{l}\text { Depths that reduces wall } \\
\text { thickness below minimum } \\
\text { allowable }\end{array}$ & 7.6 .4 \\
\hline
\end{tabular}

${ }^{1}$ Contact container manufacturer for criteria and procedure(s), or get the needed information from product literature, instruction manual(s), written recommendations, advertisements, etc.

2 Specified by manufacturer. Repair may be possible if directed by container manufacturer.

3 Exposed fibers may be coated. Contact manufacturer. 


\section{Measuring Damage}

Measuring damage is very difficult with the normal differences in the cylinder wrap thickness. Cuts tend to have a flared edge to them, which makes them also stick up on the edges. A depth gauge or dial caliper that has been sharpened to a point is needed to accurately measure the damage while bridging the gap accurately.

Sometimes, it is necessary to use a straight edge across a gouge to seek a level from which to measure. With this method you must subtract the thickness of the straight edge from your reading.

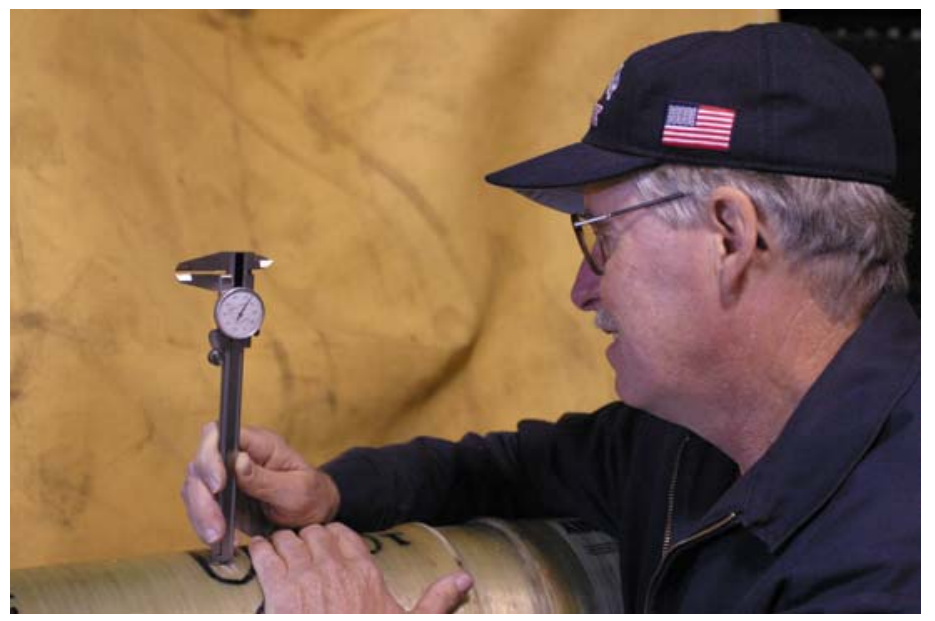

Figure 5.10 


\section{Section 6.0 Original Fuel System Installation}

This section deals with the rest of the fuel system components. It includes fuel lines, valves, fittings, pressure regulators and filling receptacles. Beyond the first stage or high-pressure regulator, systems diverge in their approach and technologies. As such there will not be any questions on underhood fuel mixing strategies, either mechanical or computer controlled.

Again, NFPA 52 is the primary resource for the installation of NGV Fuel Systems. What follows is a summary (not intended to be complete) of the major points. The latest edition should always be consulted for more detail. NOTE: NFPA 52 is designed as a minimum guideline for aftermarket conversions. The OEM's, however, build to a different set of standards that may go beyond the requirements of aftermarket converters.

\section{Fuel lines (piping) considerations:}

- When passing through a panel shall be protected by grommets or the equivalent

- Shall be mounted or supported to minimize vibrations and breakage due to strain or wear.

(This suggests stress loops or vibration loops between moving parts.)

- Fittings or joints should be located in accessible positions.

Installation of valves:

- Every cylinder must have a manual or normally closed automatically-actuated (e.g., electric), shutoff valve

- Every cylinder on an on-road vehicle must have a second valve, either manual or automatically actuated, that allows isolation of the cylinders from the rest of the fuel system*
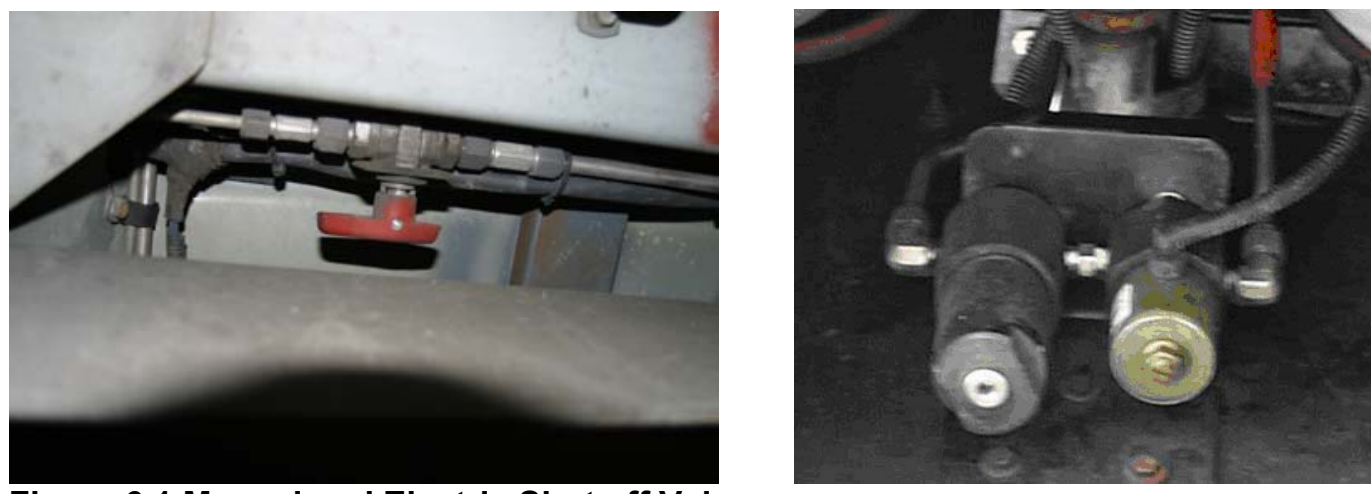

Figure 6.1 Manual and Electric Shut off Valves

- If a manual shutoff valve is used, it must not require more that 90 degrees rotation (quarter turn valve) to close ${ }^{4}$

- Access to the manual shutoff valve shall not require the use of any tool or key

- The 'quarter turn' valve must be indicated with a label or decal

- A final valve that automatically prevents the flow of gas to the engine when the engine is not running, even with the key on, is also required. NOTE: electronic fuel injectors meet this requirement

- Valves must be securely mounted and protected

- The fuel system must have a backflow check valve between the cylinders and the fill receptacle. This is incorporated into the NGV1 fill receptacle

\footnotetext{
${ }^{4}$ In the case of shutoff valves this has caused confusion in that most OEM Natural Gas Vehicles do NOT require an additional manual or quarter turn shutoff valve beyond the cylinders. The OEM's, however, build to a different set of standards that go well beyond the requirements of aftermarket converters.
} 
- The check valve mounting must be able to withstand the breakaway force (150 lbs) of the fill hose.

- There must be an additional check valve located between the cylinders and the fill point.

\section{Pressure gauges:}

While pressure gauges are optional, they do serve to give technicians the best information on system status before any service is performed. If used they:

- Cannot allow gas into the passenger compartment

- Must be equipped with shatterproof lens, and an internal pressure relief

- Must have a limiting orifice (that reduces dial fluctuation)

- Shall be securely mounted and shielded

\section{Pressure Regulators:}

- Must have a means to prevent refrigeration effects

- Must be installed so that their weight is not placed on the attached gas lines.

\section{Fueling Connections:}

- Fueling receptacles must be mounted to withstand a breakaway force beyond that specified for the dispenser hose (currently $150 \mathrm{lbs}$ )

- Must have clearance around the fueling connection to prevent interference with the fueling nozzle

Early in the development of the NGV industry, several different fill connection profiles were used.

Adapters were used to switch between them. These are no longer allowed. Today they have all been replaced by the current industry standard known as NGV1. This applies to fill nozzles on dispenser hoses and receptacles on vehicles. The design allows for three different fill pressures still found around the country: $2400 \mathrm{psi}, 3000 \mathrm{psi}$ and $3600 \mathrm{psi}$. The connector on the fill hose is usually color coded to reflect these pressures:

Green: 2400

Blue: 3000

Yellow: 3600

The unique feature is that you can always connect a lower pressure hose to a higher pressure fuel system, but you can't hook a higher pressure hose to a lower pressure system. 


\section{Section 7.0 Inspection Reporting}

Proper documentation, communication and reporting are the final steps in the process. The ability to convey your findings to both owners and agencies will impact both them and the industry.

Several checklists are available as guidelines for a complete inspection (See Appendix B). These are primarily for the benefit of the owner of the vehicle, not to report findings to the certifying agency or any other entity. However, the inspector should retain a copy for his or her records as a matter of due diligence. They all include details of the vehicle, an itemized list of inspection criteria and areas to record your results.

At the present time, CSA America is NOT requiring that copies of the inspection form be sent to them at the completion of the inspection. But, customers will want a copy as evidence of your work. Beyond the written form and results, many owners appreciate a photo attachment of the system condition. This can aid in the description of any damage and serve as a benchmark at the time of the examination. Digital photographs and electronic files can easily be added to the reports.

The last question that may arise is how to handle disposition of cylinders or systems that have potentially serious damage that would require immediate action. There are two situations where cylinders would need to be defueled or depressurized - scheduled or intended defueling such as removal or return of a out-of-date cylinder and emergency defueling in the case of confirmed or suspected Level 3 cylinder damage.

The use of atmospheric venting must be done with care. A static electrical charge can build up when releasing gas that can cause a spontaneous ignition. There are several documents that cover the necessary precautions to prevent this including CGA C-6.4 Appendix C; NFPA 52 Section 6.14 (2006) and the GTI Cylinder Care and Maintenance Handbook. All indicate that this should be done only by trained personnel. Atmospheric defueling should only be done with approval of local authorities, as natural gas (methane) is a potent greenhouse gas.

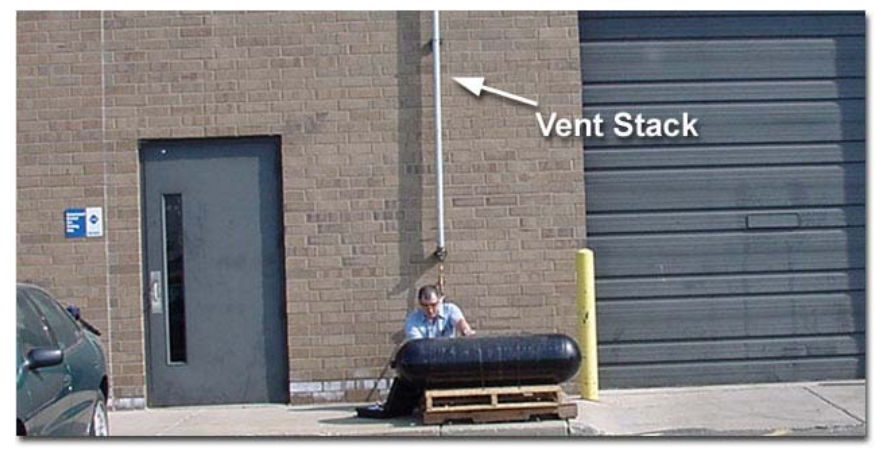

Figure 7.1 Intended defueling

Cylinder defueling is not the responsibility of the fuel system inspector, however, your advice may be helpful in guiding the authorities having jurisdiction in the proper procedures. CGA P-22 details the disposition of compressed gases and their containers. It recommends necessary training, handling, decommissioning (including removing all identifying marks), purging and rendering the cylinder unusable by drilling or cutting one or two half inch diameter holes. additional sample procedure.

Also see CGA C-6.4 Appendix C for an

CAUTION: Certain Type 4 plastic-lined cylinders may be sensitive to rapid defueling. Consult the cylinder manufacturer for guidance. PARTICULAR ATTENTION should be paid to defueling cylinders with internal electronic solenoid valves. Always follow the manufacturers' instructions. 


\title{
Appendix A
}

\section{Cylinder Manufacturers*}

CNG Cylinder Corp. In a letter dated September 14, 2006, CNG Cylinder Corp. DOT-Exemptions have been granted to $\mathrm{SCl}$, Pomona, CA. See SCI below for contact information.

Comdyne Comdyne Cylinder Co. is no longer in business. See CVEF Safety Warning dated Nov. 6, 2007 (http://www.cleanvehicle.org/technology/Comdyne Warning.pdf).

\author{
Dynetex Industries \\ 4410 46th Ave SE \\ Calgary, Alberta, Canada T2B 3N7 \\ Tel. 888-396-3835 \\ http://www.dynetek.com/cng.php \\ Faber Industrie SpA \\ Zona Industriale \\ 33043 Cividale del Friuli (Udine) - Italy \\ http://www.faber-italy.com/cng.htm
}

\author{
Lincoln Composites \\ 4300 Industrial Avenue \\ Lincoln, NE 68504 \\ Tel. 800-279-8265 \\ http://www.lincolncomposites.com/
}

Lucas Aerospace Power Equipment Co. is no longer in the CNG cylinder business. That division has changed hands several times, including TRW and AeroVantix. Some information can be obtained from the Clean Vehicle Education Foundation.

\section{Luxfer Gas Cylinders, USA \\ 3016 Kansas Avenue, \\ Riverside, California 92507, USA \\ Tel: +1 9516845110 or 1-800-764-0366 \\ http://www.luxfercylinders.com/products/cng/}

NGV Systems Inc. is no longer in business, however information can be obtained from SCl.

PST (Pressed Steel Tank Company, Inc.)

Tel. 414-476-0500

http://www.pressedsteel.com/index.html

No longer producing CNG cylinders but information is still available.

Quantum Fuel Systems Technologies

17872 Cartwright Road

Irvine, CA 92614

Tel. 949-399-4500

http://www.qtww.com

SCI (Structural Composites Industries)

325 Enterprise Place

Pomona, CA 91769

Tel. 909-594-7777

http://www.scicomposites.com/ 
Taylor-Wharton-Cylinders

521 Green Cove Road

Huntsville, AL 35803-3033

Tel: +1-256-650-9100 or 800-898-2657

http://www.taylorwharton.com

\section{OEM Manufacturers*}

Blue Bird

Daimler Chrysler

El Dorado National

Ford

Freightliner

GM

Honda

$\mathrm{NABI}$

New Flyer

Orion

Thomas Built Buses
Tel. 912-822-2091

Tel. 248-576-4117

Tel. 909-591-9557

Tel. 313-322-4771

Tel. 503-745-5219

Tel. 905-644-5020

Tel. 310-781-5718

Tel. 256-831-6155

Tel. 402-464-6611

Tel. 905-403-1111

Tel. 336-881-7243 http://www.blue-bird.com/

http://www.dcbusna.com/

http://www.enconline.com/

http://www.ford.com/

http://www.freightlinertrucks.com/

http://www.gm.com/

http://www.honda.com/

http://www.nabiusa.com/

http://www.newflyer.com/

http://www.orionbus.com/

http://www.thomasbus.com/

\section{Equipment Manufactures/Converters*}

$\begin{array}{lll}\text { BAF } & \text { Tel. 214.231.1450 } & \\ \text { Baytech } & \text { Tel. 415-949-1976 } & \underline{\text { http://www.BAFtechnologies.com }} \\ \text { Campbell-Parnell USA } & \text { Tel. 623-581-8335 } & \underline{\text { http://www.baytechcorp.com }} \\ \text { ECO Fuel Systems } & \text { Tel. 604-888-8384 } & \underline{\text { http://www.ecofuel.com/ }} \\ \text { FAB Industries } & \text { Tel. 256.831.6155 } & \underline{\text { http://www.fabind.com/ }} \\ \text { Teleflex/GFI } & \text { Tel. 519-576-4270 } & \underline{\text { http://www.teleflexgfi.com/ }} \\ \text { ITT Conoflow } & \text { Tel. 843-563-9281 } & \underline{\text { http://www.conoflow.com }} \\ \text { NaturalDrive } & \text { Tel. 801-768-2986 } & \underline{\text { http://www.naturaldrive.com/ }} \\ \text { Parker } & \text { Tel. 256-881-2040 } & \underline{\text { http://www.parker.com }} \\ \text { Sherwood Valves } & \text { Tel. 724-225-8000 } & \underline{\text { http://www.sherwoodvalve.com/ }} \\ \text { SSP Fittings } & \text { Tel. 330-425-4250 } & \underline{\text { http://www.sspfitings.com }} \\ \text { TransEco Energy } & \text { Tel. 828-654-8300 } & \end{array}$

This list is not all inclusive. Any entities wishing to be added should contact:

AFV International at (740) 205-2107 


\section{Appendix B Sample Checklists}

\section{CSA America CNG Cylinder Inspection Form}

\section{CNG CYLINDER INSPECTION FORM}

Date:

\begin{tabular}{|c|c|c|c|c|}
\hline Vehicle Make & Model & Mileage & Year & VIN\# \\
\hline & & & & \\
\hline & 1 & 2 & 3 & $\overline{4}$ \\
\hline \multicolumn{5}{|c|}{ Cylinder No. } \\
\hline \multicolumn{5}{|c|}{ Manufacturer } \\
\hline \multirow{2}{*}{\multicolumn{5}{|c|}{$\begin{array}{l}\text { Serial\# } \\
\text { Location }\end{array}$}} \\
\hline & & & \multicolumn{2}{|c|}{ Location } \\
\hline Label Serial \# & & & & \\
\hline
\end{tabular}

\begin{tabular}{|c|c|c|c|c|c|c|}
\hline & & & Pas & & $=F$ & \\
\hline NFPA 52 Sect. & CGA sect. & CNG Cylinder Examination & 1 & 2 & 3 & 4 \\
\hline $3-3$ & 7.4 .1 & Cylinder installation compliant with NFPA-52 & & & & \\
\hline $3-3$ & 7.4 .2 .4 & Rubber mounting pads in place and in good condition & & & & \\
\hline $3-3$ & 7.4 .2 .4 & Cylinder firmly restrained by the brackets (no rocking, looseness or cracks) & & & & \\
\hline $3-3$ & 7.4 .2 .4 & ALL the bracket securing bolts present and tight & & & & \\
\hline $3-3$ & 7.4 .2 .4 & Mounting brackets in good condition (not bent, no deformation) & & & & \\
\hline $3-3$ & 7.4 .2 .4 & Mounting bracket area free of damage & & & & \\
\hline $3-3$ & 7.4 .2 .4 & Check bracket-to-vehicle mounting for signs of stress & & & & \\
\hline $3-3$ & 7.4 .2 .4 & Brackets and straps corrosion free & & & & \\
\hline & 7.5 .3 & Cuts, gouges and abrasions on the cylinder are less than 0.010 inch in depth & & & & \\
\hline & 7.5 .4 .1 & No signs of cylinder exposure to fire or extreme heat & & & & \\
\hline $3-4$ & 7.4 .1 & Cylinder is properly externally vented (cylinders enclosed in vehicles only) & & & & \\
\hline & 7.5 .1 .3 & Cylinder is free of rust, corrosion or etching of outer surface & & & & \\
\hline & 7.6 .2 & External paint, composite layer or metal surface is free of bubbles or bulges & & & & \\
\hline $2-8$ & 7.8 & Valves, lines and/or Pressure Relief Device (PRD) assemblies are damage free & & & & \\
\hline $2-5$ & 7.8 & PRD is in good condition (with no visible extrusion of eutectic material) & & & & \\
\hline $3-5$ & 7.4 .2 .1 & Fuel and vent lines are properly attached to the vehicle & & & & \\
\hline & 7.3 & Vehicle history (no incidents possibly damaging the cylinder) & & & & \\
\hline & 7.11 .2 & Installation of new inspection sticker & & & & \\
\hline
\end{tabular}

Summary of examination and description of damage andor adverse findings:

Repairs or replaced brackets or other components as follows:

Cylinder Inspection Results (check one)

Return Cylinder(s) to service.

Repair Cylinder(s) as follows:



Send Cylinder(s) to Mrr. for further inspection as follows:

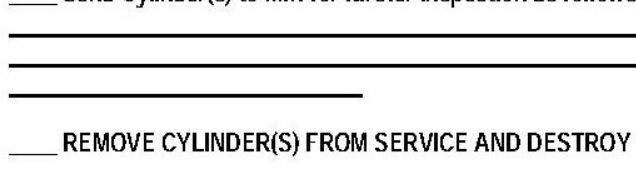

Certificate No.

Inspector Signature: 


\section{CGA Sample Inspection Form}

Vehicle Inspector Name:

Vehicle Inspector Organization:

Date:

Vehicle Mileage:

Vehicle Make and Model:

Vehicle Identification Number (VIN):

NGV Container:

Manufacturer:

Part Number: Serial Number:

Location:

NOTE-An examination form shall be filled out for each fuel container in an installation.

\begin{tabular}{|c|c|c|c|}
\hline $\bar{Y}$ & $\mathbf{N}$ & & Examination \\
\hline & & 1 & Is the container and mounting bracket area clean, free of dirt, and ready for examination? \\
\hline & & 2 & Is the container free from evidence of fire or exposure to extreme temperatures? \\
\hline & & 3 & Is the container free from indications that the container has been involved in an accident? \\
\hline & & 4 & $\begin{array}{l}\text { Has the owner been questioned about any conditions or incidents that may have caused damage to } \\
\text { the container? (Report adverse findings below.) }\end{array}$ \\
\hline & & 5 & Is the installation in compliance with applicable regulations (e.g., NFPA 52 or CSA B109)? \\
\hline & & 6 & Is this inspection being conducted before the expiration of the container service life? \\
\hline & & 7 & $\begin{array}{l}\text { Are the container service pressure markings greater than or equal to the vehicle service pressure } \\
\text { markings? }\end{array}$ \\
\hline & & 8 & $\begin{array}{l}\text { Is there a minimum of } 0.5 \text { in (12.7 mm) clearance around the container when mounted? (A minimum } \\
\text { standoff of } 0.375 \text { in [ } 9.52 \mathrm{~mm} \text { ] is recommended for shields.) }\end{array}$ \\
\hline & & 9 & If containers are enclosed in the vehicle, are they properly vented externally to the vehicle? \\
\hline & & 10 & Are fuel and vent lines properly and securely attached to the vehicle? \\
\hline & & 11 & Is the rubber pad between the mounting bracket and fuel container in place and in good condition? \\
\hline & & 12 & Is the container firmly restrained by the brackets (i.e., no rocking or looseness)? \\
\hline & & 13 & Are the bolts that secure the brackets to the vehicle present and tight? \\
\hline & & 14 & Is the mounting bracket in good condition and not bent or deformed? \\
\hline & & 15 & is the vehicle free of damage where the mounting brackets are attached? \\
\hline & & 16 & Are bolts on brackets or straps torqued to proper levels? \\
\hline & & 17 & Are the valve and/or relief device assemblies free of damage? \\
\hline & & 18 & $\begin{array}{l}\text { Are the valves and relief devices tightly seated? } \\
\text { (Caution: Do not loosen valves or relief devices while the tank is pressurized.) }\end{array}$ \\
\hline & & 19 & Are the interfaces between the valves or relief devices and the container free of leaks? \\
\hline & & 20 & If there are cuts, gouges, or abrasions present, are they less than $0.01 \mathrm{in}(0.25 \mathrm{~mm})$ deep? \\
\hline & & 21 & $\begin{array}{l}\text { Is the container free of impact damage (e.g. surface discoloration, cracked resin, chipping, loose fi- } \\
\text { bers)? }\end{array}$ \\
\hline & & 22 & Is the container free of surface dents? \\
\hline & & 23 & Is the container free of rust, corrosion, or etching of the outer surface? \\
\hline & & 24 & Is the container surface free of discoloration? \\
\hline & & 25 & Is the external paint, composite layer, or metal surface free of bubbles or bulges? \\
\hline & & 26 & Is the pressure relief device (PRD) in good condition with no visible extrusion of fusible metal? \\
\hline & & 27 & Are all relief devices in place? \\
\hline & & 28 & Are brackets or straps free of corrosion? \\
\hline & & 29 & Has the area under the straps been examined? \\
\hline & & 30 & Has a new examination sticker or stamp/mark been applied? \\
\hline & & 31 & Has the cylinder been pressurized above the applicable design standard? \\
\hline
\end{tabular}

(continued on next page) 


\section{CGA Sample Inspection Form (con't.)}

Summary of examination and description of any damage or adverse findings:

Repair or replace brackets or other components as follows:

Recommended disposition for fuel container:

\begin{tabular}{|l|l|l|}
\hline & 1 & Repair fuel container as follows: \\
\hline & 2 & Send fuel container to manufacturer for further inspection as follows: \\
\hline & 3 & Remove fuel container from service and condemn \\
\hline & 4 & Return fuel container to service \\
\hline
\end{tabular}

Signature of inspector: 


\section{CNG CYLINDER INSPECTION FORM} Model \# Mileage:

Year:

VIN\#

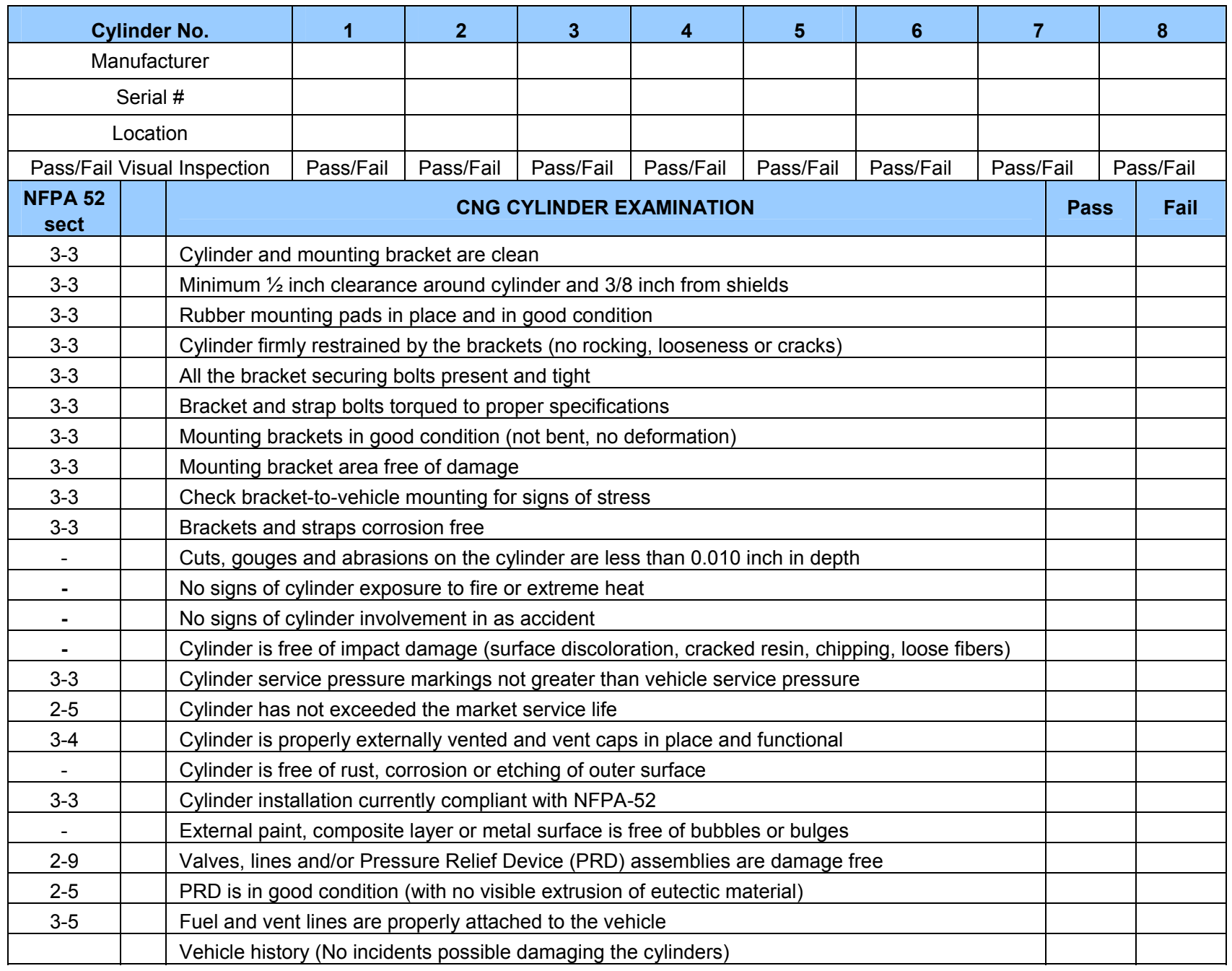

Summary of examination and description of damage and/or adverse findings:

Repair or replaced brackets or other components as follows:

\section{Cylinder Inspection Results (check one)}

$\square$ Return Cylinder(s) to Service

$\square$ Repair Cylinder \#(s) as follows:

$\square$ Send Cylinder \#(s) to Mfr. For further inspection as follows:

$\square$ REMOVE CYINDER \#(S) FROM SERVICE AND DESTROY Inspector/Tech \# 


\section{Appendix C}

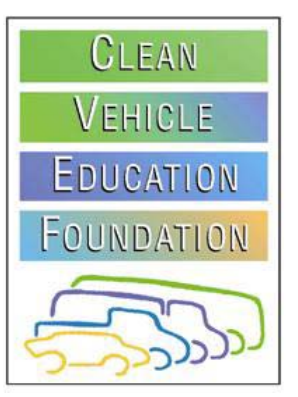

6812 Haycock Road

Falls Church, VA 22043, USA

1-703-534-6151

www.cleanvehicle.org

\section{Codes, Standards and Advisories Applicable to Natural Gas Vehicles and Infrastructure}

(N.B. This list is not all-inclusive)

\section{Document}

\begin{tabular}{l} 
NFPA 52 - Vehicular Fuel Systems Code - \\
2006 \\
\hline NFPA 57 - Liquefied Natural Gas Vehicular \\
Fuel System Code - 2002
\end{tabular}

Fuel System Code - 2002

NFPA 88A - Standard for Parking Structures -

2007

NFPA 30A - Code for Motor Fuel Dispensing

Facilities and Repair Garages - 2007

NFPA 59A - Standard for the Production,

Storage, and Handling of Liquefied Natural

Gas - 2009

SAE J1616 - Recommended Practice for

Compressed Natural Gas Vehicle Fuel - 1994

SAE J2343 - Recommended Practices for

LNG Powered Heavy-Duty Trucks- 2007

SAE J2406 - Recommended Practices for

CNG Powered Medium and Heavy- Duty

Trucks - 2002
Applicability

\begin{tabular}{l|l}
\multicolumn{2}{c}{ Comments } \\
lity
\end{tabular}

fueling facilities,

LNG and L/CNG vehicles (incl. marine) and fueling facilities

Open, enclosed, basement and

underground parking structures

Facilities dispensing both gaseous

and liquid fuels at the same facility

Site selection, design, construction,

and fire protection for LNG

facilities.

CNG motor vehicle fuel

LNG powered heavy duty trucks

CNG powered medium and heavy

duty trucks (>14,000 GVWR)
Probably single best source of guidance for CNG vehicles and fueling facilities.

Single best source of guidance for LNG vehicles and fueling facilities

No special requirements for NGVs other than reference to NFPA 52 and 57

Includes requirements of old 88B on repair garages.

Recommendations on vehicular fuel composition.

Primarily heavy truck recommendations but some maintenance facility equipment and procedures.

Published in 2002. 


\begin{tabular}{|c|c|c|}
\hline $\begin{array}{l}\text { SAE J2645 - Liquefied Natural Gas (LNG) } \\
\text { Vehicle Metering and Dispensing Systems }\end{array}$ & $\begin{array}{l}\text { LNG Vehicular Fuel Metering and } \\
\text { Dispensing. }\end{array}$ & Published in 2008 \\
\hline $\begin{array}{l}\text { Design Guidelines for Bus Transit Systems } \\
\text { Using Liquefied Natural Gas (LNG) as an } \\
\text { Alternative Fuel (3/97) }\end{array}$ & $\begin{array}{l}\text { Transit Facilities but useful reference } \\
\text { for other fleets }\end{array}$ & $\begin{array}{l}\text { FTA Report - Not only references required codes (e.g., NFPA) but also } \\
\text { suggests additional precautions and provides general information. }\end{array}$ \\
\hline $\begin{array}{l}\text { Design Guidelines for Bus Transit Systems } \\
\text { Using Compressed Natural Gas as an } \\
\text { Alternative Fuel }(6 / 96)\end{array}$ & ditto & ditto \\
\hline $\begin{array}{l}\text { Compressed Natural Gas Safety in Transit } \\
\text { Operations (10/95) }\end{array}$ & ditto & ditto \\
\hline $\begin{array}{l}\text { Liquefied Natural Gas Safety in Transit } \\
\text { Operations (3/96) }\end{array}$ & ditto & ditto \\
\hline NFPA 1 - Fire Code - 2009 & $\begin{array}{l}\text { "Adopted in jurisdictions throughout } \\
\text { North America" }\end{array}$ & May be the fire code used in your area. Check with local fire marshal. \\
\hline International Fire Code - 2006 & $\begin{array}{l}\text { "regulations governing the } \\
\text { safeguarding of life and property from } \\
\text { all types of fire and explosions } \\
\text { hazards." }\end{array}$ & Check with local fire marshal on applicability. \\
\hline $\begin{array}{l}\text { CSA B108-99 (R2006) Natural Gas Fuelling } \\
\text { Stations Installation Code }\end{array}$ & $\begin{array}{l}\text { Canadian Std. applicable to fleet and } \\
\text { public stations }\end{array}$ & \\
\hline $\begin{array}{l}\text { CSA B109-01 - Natural Gas for Vehicles } \\
\text { Installation Code }\end{array}$ & $\begin{array}{l}\text { Canadian Std. Applies to "installation, } \\
\text { servicing and repair of NG fuel } \\
\text { systems on self-propelled vehicles." }\end{array}$ & \\
\hline $\begin{array}{l}\text { ANSI NGV1-2006 - Compressed Natural Gas } \\
\text { Vehicle (NGV) Fueling Connection Devices }\end{array}$ & $\begin{array}{l}\text { CNG vehicular fueling connection } \\
\text { devices }\end{array}$ & Assures standardized nozzles and receptacles \\
\hline $\begin{array}{l}\text { ANSI NGV 2-2007 - Compressed Natural Gas } \\
\text { Vehicle Fuel Containers }\end{array}$ & CNG fuel containers & Container requirements in addition to FMVSS 304. \\
\hline $\begin{array}{l}\text { ANSI NGV3.1-1995 - Fuel System } \\
\text { Components for Natural Gas Powered } \\
\text { Vehicles }\end{array}$ & $\begin{array}{l}\text { Fuel system components for NGVs } \\
\text { (excludes LNG components upstream } \\
\text { of vaporizer) }\end{array}$ & Primarily for converted vehicles. \\
\hline $\begin{array}{l}\text { ANSI NGV4.1/ CSA } 12.5-1999-\text { NGV } \\
\text { Dispensing Systems }\end{array}$ & $\begin{array}{l}\text { CNG vehicular fuel dispensing } \\
\text { systems }\end{array}$ & \\
\hline
\end{tabular}


Document

ANSI NGV4.2/CSA 12.52 -1999 - Hoses for

NGVs and Dispensing Systems

ANSI NGV4.4/CSA 12.54 -1999 - Breakaway

Devices for Natural Gas Dispensing Hoses

and Systems

ANSI NGV4.6/CSA 12.56 -1999 - Manually

Operated Valves for Natural Gas Dispensing Systems

ANSI NGV4.8/CSA 12.8 -2002 - Natural Gas

Vehicle Fueling Station Reciprocating

Compressor Guidelines

ANSI PRD1-1998 (with 1999 \& 2007 addenda)

- Basic Requirements for Pressure Relief

Devices for Natural Gas Vehicle Fuel

Containers

CGA C-6.4-2007 - Methods for External Visual

Inspection of Natural Gas Vehicle Fuel

Containers and Their Installations

49 CFR 178.56 - Specification 4AA welded

steel cylinders

49 CFR 178.57 - Specification 4L welded

insulated cylinders

49 CFR 571.304, FMVSS 304 - Compressed

Natural Gas Fuel Container Integrity

49 CFR 571.303, FMVSS 303 - Fuel System

Integrity of Compressed Natural Gas Vehicles

49 CFR 393.65, FMCSR - All Fuel Systems
Applicability

Comments

CNG dispenser and vehicular hose assemblies

CNG dispenser shear valves and

fueling hose emergency breakaway

shutoff devices

Manually operated CNG valves,

excluding cylinder shut-off valves

Compressor packages containing

reciprocating compressors used in

CNG fueling station service.

Pressure Relief Devices for CNG Fuel

Containers

CNG vehicular fuel containers

Referenced in ANSI NGV2

CNG cylinders for fueling stations.

LNG vehicular fuel tank requirement

called out in NFPA 57

CNG motor vehicle fuel containers

CNG vehicles $\leq 10,000 \mathrm{lbs}$. GVWR

and school buses

Commercial vehicles in interstate

commerce
Generally not used for new CNG fueling stations. ASME vessels now generally used.

Option is meeting ASME Boiler and Pressure Vessel Code.

DOT Federal Motor Vehicle Safety Standard for CNG motor vehicles.

DOT Federal Motor Vehicle Safety Standard for crash test of light duty vehicle and school bus CNG fuel systems.

DOT Federal Motor Carrier Safety Regulations. May have been adopted by states for intrastate application. Wasn't written w NGVs in mind but may be legally applicable. 
Document

40 CFR 80.33 - Controls applicable to natural gas retailers and wholesale purchaser-

consumers

40 CFR 86.098-8 - Emission standards for 1998 and later model year light-duty vehicles

ASME Boiler and Pressure Vessel Code,

Section VIII (Pressure Vessels)

\section{CA Code of Regulations, Title 13, Div 2, Ch 4,}

\section{Article 2}

CA Code of Regulations, Title 8, Div 1, Ch 4,

Subchapter 1

CA Code of Regulations, Title 13, Div 3, Ch 5,

Article 3, Sec 2292.5

TX Administrative Code, Title 16, Part 1
Applicability

Retailer and wholesale purchaserconsumers of NG

Light-Duty Vehicles

Sections applicable to LNG containers

used on vehicles and in fueling

stations. Sections applicable to

containers used in CNG fueling

stations.

Fuel systems using LNG in $13 \mathrm{CCR}$

935, CNG in 13 CCR 934

CNG and LNG Storage Tanks

CNG sold in CA

CNG regulations in Chapter 13, LNG

regulations in Chapter 14
Comments

EPA 1.2 gm limit on atmospheric venting per refueling.

Requires NGV1 receptacles.

CA vehicle requirements

CA fuel storage requirements

CA CNG composition requirements

TX requirements

Availability:

National Fire Protection Association (NFPA) documents - contact NFPA at 1-800-344-3555 or http://catalog.nfpa.org

Society of Automotive Engineers (SAE) documents - contact SAE at 774-726-0790 or www.sae.org/products

Federal Transit Administration (FTA) documents - contact William Hathaway at 617-494-2081 or the National Technical Information Service at 703-605-6050 or www.ntis.gov

Uniform Fire Code - Contact Western Fire Chiefs Association/Uniform Fire Code Association at 760-723-6911 or www.wfca.com/ufca or buy from a bookstore, such as Amazon.Com

International Fire Code - Contact International Codes Council at 703-931-4533 or www.intlcode.org

Canadian Standards Association (CSA) documents - Contact CSA at 1-800-463-6727 or www.csa.ca

ANSI NGV documents - May be purchased from CSA at http://www.csa-intl.org/onlinestore/getcatalogdrilldown.asp?Parent=0\&k=3\&l=1 or

ANSI at http://webstore.ansi.org/ansidocstore/default.asp 
Compressed Gas Association (CGA) documents - Contact CGA at 703-788-2700 or www.cganet.com

Code of Federal Regulations (CFR) - Can be obtained on the web at www.access.gpo.gov

California Code of Regulations (CCR) - Can be obtained on the web at http://ccr.oal.ca.gov

Texas Administrative Code - Can be obtained on the web at http://info.sos.state.tx.us:80/pub/plsql/readtac\$ext. ViewTAC

ASME Boiler and Pressure Vessel Code - Contact ASME at 800-843-2763 or www.asme.org updated 9/11/08 


\section{Appendix D}

There are 60 questions on the test that are graded toward certification although there may be additional sample questions inserted for evaluation but not graded. They are proportioned according to the Objectives as follows:

Percentage

Of Coverage

Section 1 Preparation for Inspection

Section 2 Determine Inspection Requirements

Section 3 Pressure Relief Device Inspection

Section 4 Physical Inspection of Brackets and HP Components

Section 5 Physical Assessment of Cylinders

Section 6 CNG Fuel System Installation

Section 7 Inspection Reporting
$13 \%$

$9 \% \quad 5$

$7 \%$

$18 \%$

$38 \%$

$10 \%$

$5 \%$

$100 \%$

\section{Number of \\ Questions}

8

4

11

23

6

3

60

The test is offered throughout the year as a computer-based exam offered at designated centers all across the country and as a paper and pencil exam offered during scheduled, semi-annual exam sessions. Candidates for certification may download the personnel certification guide and application form from CSA America's website at:

http://www.csa-america.org/personnel_certification/cng_certification/.

Completed application forms can be submitted via email to: personnelcertification@csa-

america.org or by faxing to (216) 520-8979. Once the application and payment are received and processed, CSA will send the candidate information to the test vendor. The test vendor will email the candidate the "Notice to Schedule" or NTS which includes instructions on scheduling their exam session. Once the candidate receives their NTS, they will be able to register for the exam at the test site/date they choose. Candidates must submit the scheduling request at least 10 business days prior to the requested examination date. Testing sites are located throughout the United States and are normally within a short driving distance from most potential inspectors. 


\section{Taking the Test}

All of the questions are direct multiple choice or of the type "given a scenario... what would you recommend." Unlike the ASE tests, there are no "True/False" questions, no "Technician A or Technician B" questions, no "fill in the blank" and no negative questions ( e.g.: "All of the following are true EXCEPT" or "none of the above"). Each question will have only one correct answer.

You will have more than enough time and there is no need to feel rushed, but as with all test taking, keep track of the time and monitor your progress. Read each question thoroughly and carefully. Answer all of the questions you are confident of quickly and then go back and concentrate on those that you need more time to think about. Make sure you attempt an answer on all of the questions.

\section{Sample Questions}

1. The cylinder service pressure is the pressure measured at

a. 70 deg. $F$

b. 140 deg. $F$

c. 180 deg. $F$

d. Ambient Temperature

2. The primary purpose of the liner in a Type 4 , all composite cylinder is to

a. prevent the absorption of water vapor

b. absorb the gas

c. prevent gas leakage

d. contain gas pressure

3. An undamaged CNG cylinder with a service pressure of 3,600 psig is designed with a safety factor so as not to rupture

a. in excess of 4500 psig

b. in excess of 8,000 psig

c. in excess of 15,000 psig

d. will not rupture at any pressure

4. In a Type 4 all-composite cylinder, the portion of pressure load due to internal pressure, taken up by the plastic liner is
a. $100 \%$
b. $10 \%$
c. $5 \%$
d. $0 \%$

5. A composite wrap consists of

a. fibers embedded in a resin

b. a resin system

c. a hoop wrapped resin system

d. metal cords in a rubber base 
6. The pipe or tubing attached to the pressure relief device is commonly known as

a. the pressure overflow line

b. the high pressure line

c. the vent line

d. none of the above

7. The agency having jurisdiction over the FMVSS 304 standard is

a. the National Highway Traffic Safety Administration (NHTSA)

b. the Federal Transit Administration (FTA)

c. the U.S. Department of Energy (DOE)

d. the Clean Cities network

8. What is the useful life of cylinders made to the 1992 version of the NGV 2 standard?

a. 5 years

b. 15 years

c. 30 years

d. indefinite

9. NGV 2 recommends that, as a minimum, cylinders should be subject to a detailed visual inspection
a. every year
b. every two years
c. during every refueling
d. every three years

10. In addition to setting forth cylinder inspection requirements, the CGA C-6.4 standard also addresses the following topic:

a. cylinder installation

b. fueling connectors

c. fueling station ground storage

d. emission requirements

11. A cylinder mounted inside a vehicle must be

a. protected from road debris

b. mounted in locations that minimize damage

c. vented to the outside

d. NFPA 52 does not allow this type of installation 
12. During the detailed visual inspection a cut on the cylinder was found. What would you recommend?

a. the cylinder should be condemned

b. the cylinder manufacturer should be contacted

c. the level of damage should be determined

d. the cylinder should be immediately defueled

13. To perform the general visual inspection, how much training is required?

a. ASE Certification

b. two years of hands-on training

c. understanding and knowledge of cylinder damage

d. CSA Fuel System Inspection certification

14. The primary inspection method in the NGV 2 and the DOT FMVSS 304 standard is

a. detailed visual inspection

b. hydrostatic testing

c. ultrasonic testing

d. acoustic emission testing

15. One of the major safety concerns with venting natural gas from a cylinder is

a. overheating the vent pipe

b. static build-up

c. pollution of the environment

d. cuts due to high pressure

16. You are inspecting a school bus with cylinders mounted in the undercarriage and notice the road clearance is just above the 9 inch minimum. It's obvious that if the bus had a flat tire the clearance would be below that. What would you recommend?

a. As long as the clearance is about 9 inches, this is not a defect.

b. Level 1 defect, record the finding and return the bus to service

c. Level 2 defect, this must be repaired

d. Level 3 defect, defuel and condemn the cylinder

17. An older Type 4 fiberglass wrapped cylinder is mounted under the bed of a cargo van used to transport car batteries for recycling. During inspection, there is evidence of chemical attack on the cylinder.

Based on this information, what action would you recommend?

a. Clean any residue from the cylinder after the inspection is completed

b. Remove the cylinder for visual inspection

c. Contact the container manufacturer for guidance

d. Defuel the cylinder immediately and condemn it. 
18. Refer to the illustration.

The driver of a converted passenger van states that he drove over a large curb and heard a loud sound. The abrasion is inspected and found to have a depth of 0.060 inches.

What is the level of damage and recommended action?

a. Level 1: no action required

b. Level 2: contact the cylinder manufacturer for its recommend action

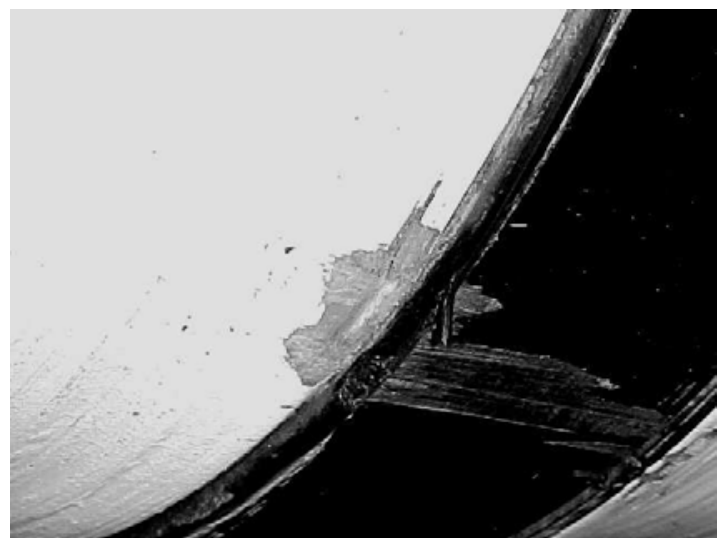

c. Level 3: contact the vehicle manufacturer for a recommend repair procedure

d. Level 3: condemn the cylinder

19. A surface cut in a cylinder is 2 inches long and 0.040 inches deep. What should be done?

a. condemn the cylinder

b. consult the cylinder manufacturer's guidelines to determine the damage level

c. remove the cylinder and consult the cylinder manufacturer's guidelines

d. approve the cylinder for service

20. Refer to the illustration.

Identify the type of cylinder.
a. Type 1
b. Type 2
c. Type 3
d. Type 4

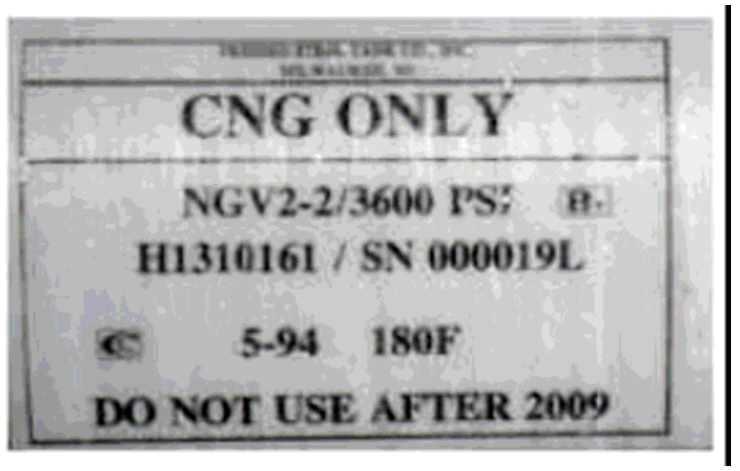

ANSWER KEY: 1.a, 2.c, 3.b, 4.d, 5.a, 6.c, 7.a, 8.b, 9.d, 10.a, 11.c, 12.c, 13.c, 14.a, 15.b, 16.c, 17.d, 18.d, 19.b, 20.b 
Appendix J - Cylinder and Vehicle Manufacturers' Inspection Requirements

In order to meet NETL's requirements that the final report accompanying form 241.3 be uploaded as one document in pdf file, this appendix would be unacceptably compromised (all files are listed by Adobe in alphabetical order, rather than under appropriate subfolders). Therefore this appendix has not been included.

Please contact CVEF for a CD copy of the Cylinder and Vehicle Manufacturers' Inspection Requirements. 


\section{Appendix K - NGV Incidents in CVEF Files as of 1-5-09}

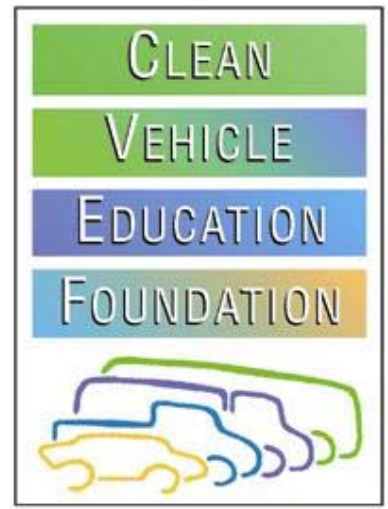

The Clean Vehicle Education Foundation and its predecessor organization have been collecting information on natural gas vehicle "incidents" as long as they have been used in the US. In most cases detailed information about the incident is available in our files. This information is used by the NGV industry to improve codes and standards for the manufacture, fueling and use of NGVs and to help ensure the vehicles' safety of operation.

Incident information in our files is from publicly available sources or has been provided to CVEF voluntarily, with the understanding that it is intended only for the use of the industry and appropriate government agencies to improve the safety of natural gas vehicles.

\begin{tabular}{|c|c|c|}
\hline File \# & Date & Description \\
\hline $84-1$ & 7/4/84 (?) & $\begin{array}{l}\text { CNG Billings (MT) Yellow Cab (1980 Chevrolet Impala) broadsided } \\
\text { gasoline Chevrolet pickup. Driver's side gasoline tank on pickup } \\
\text { split, pouring gasoline over front of taxi resulting in fire. No leaks or } \\
\text { damage to CNG system. Minor driver injuries. }\end{array}$ \\
\hline $85-1$ & 6/19/85 & $\begin{array}{l}\text { Bi-fuel } 1983 \text { AMC Eagle owned by Philadelphia Electric Co. Gaso- } \\
\text { line fuel line failed leading to engine fire. Apparently CNG fuel line } \\
\text { ruptured in fire, causing explosion and feeding fire. Two minor inju- } \\
\text { ries. }\end{array}$ \\
\hline $87-1$ & $\begin{array}{l}\text { Winter } \\
1987\end{array}$ & $\begin{array}{l}\text { Bi-fuel } 1976 \text { Ford hit another vehicle and a utility pole in Vancouver } \\
\text { BC. Small gasoline fire ensued. No damage to NG system. Seri- } \\
\text { ous injuries to both drivers. }\end{array}$ \\
\hline $90-1$ & $7 / 6 / 90$ & $\begin{array}{l}\text { Fire in Central Netherlands Transport bus garage destroyed } 35 \\
\text { buses (including two fully fueled CNG buses). "The safety systems } \\
\text { worked perfectly" and all cylinders were intact after the fire. Appar- } \\
\text { ently CNG buses did not cause or contribute to fire. }\end{array}$ \\
\hline 93-1 & $8 / 26 / 93$ & $\begin{array}{l}\text { CNG Cylinder Co. type } 2 \text { aluminum cylinder with fiberglass wrap } \\
\text { ruptured in Southeastern Michigan Gas Co. Chevrolet Corsica, } \\
\text { puncturing the gasoline tank and causing a fire. Escaping NG fed } \\
\text { fire. Substantial pre-existing damage to wrap and overpressuriza- } \\
\text { tion to } 4000 \text { psi found. } 1983 \text { DOT exemption cylinder with no re- } \\
\text { cord of required three year hydro test and visual inspection. One } \\
\text { serious injury. }\end{array}$ \\
\hline $93-2$ & $12 / 93$ & $\begin{array}{l}\text { Cherry Creek, CO School District bus leaked gas during storage in } \\
\text { indoor garage. Overhead heaters caused fire and small explosion. } \\
\text { All seven tanks vented and fed fire. Speculation that DOT 3A } 2400 \\
\text { psi cylinders may have been overfilled at local } 3000 \text { psi station (not } \\
\text { NGV1 connectors) and additional expansion when heaters came } \\
\text { on could have failed } 3750 \text { psi cylinder burst disc. No injuries. }\end{array}$ \\
\hline $93-3$ & $\begin{array}{l}1993- \\
1996\end{array}$ & $\begin{array}{l}\text { Hercules engines in school buses and shuttle buses. Various en- } \\
\text { gine (throttle body, sensors, computers, head gaskets, low com- } \\
\text { pression, melted pistons) and fuel problems. }\end{array}$ \\
\hline $94-1$ & $1-15-94$ & Las Vegas transit bus drove away w/o disconnecting fueling con- \\
\hline
\end{tabular}




\begin{tabular}{|c|c|c|}
\hline & & $\begin{array}{l}\text { nector. Breakaway at dispenser did not function. Check valve on } \\
\text { outside of bulkhead connector pulled loose, allowing gas flow which } \\
\text { ignited. No injuries but bus heavily damaged. }\end{array}$ \\
\hline $94-2$ & $\begin{array}{l}1 / 18 \text { and } \\
2 / 1 / 94\end{array}$ & $\begin{array}{l}\text { Comdyne (type 3) tank ruptured during refueling in PG\&E and Min- } \\
\text { negasco } 1992 \text { GMC Sierra pickup trucks. Caused by stress corro- } \\
\text { sion cracking of cylinder composite wrap, from exposure to battery } \\
\text { acid dripped through bolt holes in truck bed. No fires, two iniuries. }\end{array}$ \\
\hline $94-3$ & $2 / 94$ & $\begin{array}{l}\text { CNG powered bus caught fire in a maintenance garage in Bryan, } \\
\text { TX (Brazos Transit). Fire caused by short circuit igniting leaking } \\
\text { power steering fluid. CNG vented properly. "Gas ignited in a fire- } \\
\text { ball against the door, but did no damage." }\end{array}$ \\
\hline 94-4 & $3 / 23 / 94$ & $\begin{array}{l}\text { Sun Oil Co. fueling station in Philadelphia area PRV automatically } \\
\text { relieved to reduce system pressure. No fire or equipment failure. } \\
\text { Noise of valve relieving brought the fire department and media at- } \\
\text { tention. }\end{array}$ \\
\hline $94-5$ & 4/94 (?) & $\begin{array}{l}1992 \text { CNG pickup owned by Energas Co. was broadsided in Mid- } \\
\text { land, TX. Tanks, valves and fuel lines all remained intact. One } \\
\text { tank supporting strap broke. No injuries or fire. }\end{array}$ \\
\hline $94-6$ & $10 / 94$ & $\begin{array}{l}\text { SaskEnergy CNG pickup hit by oncoming car. The two steel Faber } \\
70 \text { liter cylinders mounted in the truck box stayed in place. } \\
\text { Threaded connection stripped off valve and leaked. No fire or in- } \\
\text { jury noted. }\end{array}$ \\
\hline $94-7$ & $\begin{array}{l}1994- \\
1996\end{array}$ & $\begin{array}{l}\text { Widespread Mirada PRD leakage failures caused by O-ring extru- } \\
\text { sion, low eutectic melt temperature, ice formation and over torquing } \\
\text { causing premature trigger failure. }\end{array}$ \\
\hline $95-1$ & $\begin{array}{l}1995- \\
1997\end{array}$ & $\begin{array}{l}\text { EDO LiteRider (type } 4 \text { ) tank leaks from o-rings at the valve/boss } \\
\text { interface, from the valve/liner interface and cracking of the liner. } \\
\text { Leaks noted in many different transit applications throughout North } \\
\text { America. }\end{array}$ \\
\hline $96-1$ & $2 / 13 / 96$ & $\begin{array}{l}\text { A gas recovery vessel ruptured at a Columbia Gas CNG fueling } \\
\text { station in Toledo, OH. The relief valve on the pressure vessel } \\
\text { failed to operate, probably because water had collected in the vent } \\
\text { pipe and rendered it inoperable. Explosion and fire. No injuries. }\end{array}$ \\
\hline $96-2$ & $4 / 3 / 96$ & $\begin{array}{l}\text { A 12-year old aluminum-lined Type } 2 \text { cylinder produced by NGV } \\
\text { Systems ruptured on a pick-up truck at Alabama Gas Co. No inju- } \\
\text { ries were reported. The cylinder was suspected of having external } \\
\text { physical and ultraviolet (UV) damage as well as being overpressur- } \\
\text { ized. }\end{array}$ \\
\hline $96-3$ & $6 / 3 / 96$ & $\begin{array}{l}\text { A Comdyne Type } 3 \text { cylinder in a converted Chevrolet van owned } \\
\text { and operated by Crown Services ruptured in Houston, Texas. No } \\
\text { serious injuries reported other than temporary loss of hearing. }\end{array}$ \\
\hline $96-4$ & 8/21/96 & $\begin{array}{l}\text { Rupture of one of } 10 \text { EDO cylinders on LACMTA Neoplan bus. No } \\
\text { one on board and no fire or injuries. Extensive damage to bus. } \\
\text { Determined that some sort of impact had damaged tank. Tanks } \\
\text { were mounted under the bus and not protected from damage. } \\
\text { Aluminum panels were placed under underfloor tanks to provide } \\
\text { future protection. }\end{array}$ \\
\hline $96-5$ & $1996-$ & $5^{\prime \prime}$ \\
\hline
\end{tabular}




\begin{tabular}{|c|c|c|}
\hline & 1997 & \\
\hline $97-1$ & $6 / 20 / 97$ & $\begin{array}{l}\text { A Comdyne Type } 3 \text { cylinder on a United Airlines ground vehicle } \\
\text { failed in Los Angeles, apparently due to battery acid dripping on } \\
\text { cylinder. }\end{array}$ \\
\hline $97-2$ & $10 / 23 / 97$ & $\begin{array}{l}\text { LAMTA bus electrical fire in engine compartment. PRD "operated } \\
\text { as designed... however, the released fuel ignited, resulting in a } \\
\text { rapid, highly visible burn-off. All CNG tanks...are intact, and the } \\
\text { fire does not appear to have been caused by the CNG fueling sys- } \\
\text { tem." No injuries. }\end{array}$ \\
\hline $98-1$ & $5 / 20 / 98$ & $\begin{array}{l}1991 \text { Honda Accord converted to bi-fuel impacted by another vehi- } \\
\text { cle traveling "at nearly } 160 \mathrm{~km} / \mathrm{h} \text {." Honda was stopped at Peace } \\
\text { Arch border crossing between BC and WA state. Ensuing fire fed } \\
\text { by gasoline. } 50 \text { liter CNG cylinder stayed intact and secure in its } \\
\text { brackets. Two fatalities. }\end{array}$ \\
\hline $99-1$ & 1999 & Ford CNG vehicle fuel-fill receptacle leakage. \\
\hline $99-2$ & $\begin{array}{l}8 / 10 / 99 \\
\text { and } \\
12 / 22 / 99\end{array}$ & $\begin{array}{l}\text { Engine fires on El Dorado buses at BWI airport. CNG vented } \\
\text { properly. Vent line running through engine compartment melted } \\
\text { and CNG fed fire. No injuries. }\end{array}$ \\
\hline $99-3$ & 1999 & $\begin{array}{l}\text { Canco filter housing failures at the Los Angeles County MTA and } \\
\text { City of Mesa. }\end{array}$ \\
\hline $99-4$ & 10/8/99 & $\begin{array}{l}\text { Knox-Western compressor high-pressure intercooler failure at the } \\
\text { Jackie Gleason bus garage in Brooklyn, NY. No injuries or fire. }\end{array}$ \\
\hline $99-5$ & 10/15/99 & $\begin{array}{l}\text { NGV1 nozzle adapter to Hansen coupling separated (unscrewed), } \\
\text { Laclede Gas. Minor injury, no fire. }\end{array}$ \\
\hline $99-6$ & 10/31/99 & $\begin{array}{l}\text { Comdyne Type } 3 \text { cylinder rupture on } 1996 \text { Dodge van, Enbridge } \\
\text { Consumers Gas Co, Toronto, ON. No injuries or fire. }\end{array}$ \\
\hline $99-7$ & $11 / 23 / 99$ & $\begin{array}{l}\text { High pressure release of CNG at Jackie Gleason bus garage in } \\
\text { Brooklyn, NY. Temporary flexible gasket in outdoor modulating } \\
\text { valve failed. Minor injuries to three NYC Transit employees. No } \\
\text { fire. }\end{array}$ \\
\hline $99-8$ & $12 / 24 / 99$ & $\begin{array}{l}\text { Mirada Gen. } 2.5 \text { PRD malfunctioned (caused by ice) on New Flyer } \\
\text { bus with Lincoln cylinders in State College, PA, allowing unin- } \\
\text { tended CNG venting. No fire or injuries. }\end{array}$ \\
\hline $99-9$ & $\begin{array}{l}12 / 16(?), \\
26 \text { and } \\
27 / 99\end{array}$ & $\begin{array}{l}\text { Mirada Gen. } 2.5 \text { PRDs malfunctioned on Akron RTD Orion buses } \\
\text { with } \mathrm{SCl} \text { cylinders in Akron, OH, allowing unintended CNG venting. } \\
\text { No fire or injuries in first two incidents. Explosion and one minor } \\
\text { injury in third incident. }\end{array}$ \\
\hline $00-1$ & $2 / 5 / 00$ & $\begin{array}{l}\text { FuelMaker C3 VRA overpressure sensing system clogged, allowing } \\
\text { overpressurization of CNG cylinder on delivery truck being fueled in } \\
\text { Montreal. One of four Faber Type } 1 \text { cylinders ruptured. No fire or } \\
\text { injuries. }\end{array}$ \\
\hline $00-2$ & $3 / 1 / 00$ & $\begin{array}{l}\text { Chevrolet Cavalier CNG container ruptured at Transport Canada's } \\
\text { Blaineville, PQ, contract test facility. Vehicle was being prepared } \\
\text { for CMVSS crash test. Cylinder was not completely purged of } \\
\text { CNG (air used as purge medium), then refilled to } 3777 \text { psi with } \\
\text { compressed air. Solenoid valve was then activated to drain air to } \\
3600 \text { psi. Electric arc from solenoid valve inside the tank caused } \\
\text { the explosion. Three fatalities. }\end{array}$ \\
\hline
\end{tabular}




\begin{tabular}{|c|c|c|}
\hline $00-3$ & $5 / 11 / 00$ & $\begin{array}{l}\text { Eleven foot, six inch tall Command bus shuttling race goers around } \\
\text { Belmont Park attempted to go under a 9' } 6 \text { " underpass. The entire } \\
\text { supporting frame of the roof mounted fuel storage system was } \\
\text { pushed back approximately } 10 \text { feet. A high pressure fuel line de- } \\
\text { tached causing a violent decompression of the entire fuel storage } \\
\text { system. As the released gas rose it was trapped by the overpass } \\
\text { and ignited by a damaged underpass light fixture. Three confirmed } \\
\text { minor injuries. }\end{array}$ \\
\hline $00-4$ & $6 / 20 / 00$ & $\begin{array}{l}\text { Circle Seal RV 99-273 PRD on Lincoln cylinder leaked on initial fu- } \\
\text { eling of new New Flyer bus at NYC Transit. Fueler heard whistle } \\
\text { and hit ESD. PRD replaced and bus put back in service. No inju- } \\
\text { ries, no damage. Bus had been refueled six times en route to } \\
\text { NYC. Cause of leakage unknown as yet. }\end{array}$ \\
\hline $00-5$ & $6 / 22 / 00$ & $\begin{array}{l}\text { Fire broke out in the engine compartment of a } 1998 \text { El Dorado } \\
\text { shuttle bus, operated by Dollar Rent A Car, at Denver International } \\
\text { Airport. Cummins engine and stainless vent line in this bus (see } \\
\text { BWI incidents above). CNG cylinders vented as designed, but } \\
\text { loose connection at "Y" in vent line popped off during venting, al- } \\
\text { lowing gas to feed the fire. }\end{array}$ \\
\hline $00-6$ & $6 / 23 / 00$ & $\begin{array}{l}\text { Engine compartment fire in a } 1995 \text { LACMTA Neoplan bus led to } \\
\text { venting of CNG, which fueled the fire. Damage was estimated at } \\
\text { between } \$ 10,000 \text { and } \$ 15,000 \text {, Two minor injuries. Source of fire } \\
\text { was separation of flexible and solid exhaust pipe connection (clamp } \\
\text { let go). Exhaust caused fiberglass cowling on back to bus to catch } \\
\text { fire. }\end{array}$ \\
\hline $00-7$ & $10 / 00$ & $\begin{array}{l}\text { Blue Bird recalled } 134 \text { of its } 1992-93 \text { CNG buses to replace PRDs, } \\
\text { some of which may vent unexpectedly. The original Superior valve } \\
\text { apparently was both temperature and pressure actuated. It re- } \\
\text { lieved at } 4000 \text { psi and was replaced with a valve that relieved at } \\
5000 \text { psi. }\end{array}$ \\
\hline $00-8$ & $12 / 14 / 00$ & $\begin{array}{l}\text { Type } 1 \text { cylinder failed, probably from overpressurization. Four } \\
2400 \text { psi cylinders were mounted in bed of NIPSCO } 1992 \text { con- } \\
\text { verted S10 meter reading truck. Speculate that multiple failures of } \\
\text { pressure regulating and relief systems allowed dispensing of } 6000 \\
\text { psi gas, failing cylinder during fueling. No injuries, but damage to } \\
\text { truck and fueling station. }\end{array}$ \\
\hline $00-9$ & Early 2000 & $\begin{array}{l}\text { Fire near control panel in Wisconsin CNG station. No injuries. Gas } \\
\text { turned off and fire burned out. }\end{array}$ \\
\hline 01-1 & $3 / 13 / 01$ & $\begin{array}{l}\text { Blowdown (recovery) tank regulator failed and overpressurized } \\
\text { tank which ruptured. PRV did not function. Tank designed for } 200 \\
\text { psi - it failed as it should have, perhaps around } 800 \text { psi. Location } \\
\text { was the New Flyer bus manufacturing plant in Crookston, MN. No } \\
\text { injuries. Small fires put out by local fire department. Property } \\
\text { damage to fueling station and local area. }\end{array}$ \\
\hline $01-2$ & $3 / 29 / 01$ & $\begin{array}{l}\text { DOT 3AA, } 3600 \text { psi, } 91 / 4 \times 58 \text { buffer tank ruptured at slow fill station } \\
\text { at Tumwater School District in Tumwater, WA at 5:13 a.m. Appar- } \\
\text { ently buffer tank was not overpresssurized, so must have been de- } \\
\text { fect in cylinder although hydro tested in } 1998 \text {. Supply line to com- }\end{array}$ \\
\hline
\end{tabular}




\begin{tabular}{|c|c|c|}
\hline & & $\begin{array}{l}\text { pressors was sheared and gas caught fire. Put out by fire depart- } \\
\text { ment. No injuries but PD. }\end{array}$ \\
\hline $01-3$ & $5 / 2 / 01$ & $\begin{array}{l}\text { LACMTA CNG transit bus hijacked. After two-mile chase bus col- } \\
\text { lided with minivan and UPS CNG delivery truck. Driver of minivan } \\
\text { killed. No damage to CNG systems on bus or truck. No mention of } \\
\text { vehicles being CNG-powered in press. }\end{array}$ \\
\hline $01-4$ & $8 / 01$ & $\begin{array}{l}\text { Shook PRD on SCI cylinder on Sacramento RTD "trolley" replica } \\
\text { unintentionally vented during fueling. Other Shook PRDs on all } \\
\text { four RTD trolleys showed initial symptoms of a similar problem, ex- } \\
\text { trusion of the eutectic material from the valve. }\end{array}$ \\
\hline $02-1$ & Summer/02 & $\begin{array}{l}\text { NYSDOT Honda GX struck from behind by tanker truck in NYC. } \\
\text { Left rear corner crushed to B-pillar. No leak or rupture of the CNG } \\
\text { fueling system. Driver walked away with minor injuries. }\end{array}$ \\
\hline $02-2$ & $7 / 15 / 02$ & $\begin{array}{l}\text { El Dorado bus operated by San Gabriel Transit caught fire and was } \\
\text { totally destroyed. No injuries. Origin was an oil fire, and after } \\
\text { about } 2 / 3 \text { of the bus was burned, the CNG tanks vented and con- } \\
\text { tributed to the fire. Believed to be Cummins enqine. }\end{array}$ \\
\hline $02-3$ & $9 / 24 / 02$ & $\begin{array}{l}\text { WI state-owned } 1998 \text { Ford Crown Vic CNG PST tank ruptured in } \\
\text { vehicle fire. Apparently Superior PRD did not vent gas. Three } \\
\text { other tanks did not explode. No injuries but local PD. }\end{array}$ \\
\hline $02-4$ & $2002 / 2003$ & $\begin{array}{l}\text { High-capacity NexGen LNG vehicular fuel tanks have lost vacuum } \\
\text { in the insulating section of the double-walled tanks faster than ex- } \\
\text { pected. NexGen is working with tank users to repump the vacuum } \\
\text { on-site and to set up periodic maintenance to assure vacuum is not } \\
\text { lost in the future. }\end{array}$ \\
\hline 03-1 & $1 / 03$ & $\begin{array}{l}\text { A Mirada PRD ruptured on a Bi-State (St. Louis) } 40 \text { foot Neoplan } \\
\text { CNG bus in an inside vehicle wash area. The PRD failure was ap- } \\
\text { parently caused by water and ice inside the vent tube, which was } \\
\text { missing its exterior cap. No injuries or damage. }\end{array}$ \\
\hline $03-2$ & $4 / 14 / 03$ & $\begin{array}{l}\text { A MARTA (Metropolitan Atlanta Rapid Transit Authority) CNG bus } \\
\text { caught fire and completely burned. The fire originated in a rear tire } \\
\text { and spread throughout the bus. PRDs on the roof-mounted CNG } \\
\text { tanks functioned as designed, venting CNG above the bus where it } \\
\text { caught fire. The bus was completely destroyed as the fire burned } \\
\text { itself out. No injuries. }\end{array}$ \\
\hline $03-3$ & 2003 & $\begin{array}{l}\text { Wear on the outer surface of NGV1 CNG receptacles, variously } \\
\text { described as "mushrooming," or "Brinelling" has been found on re- } \\
\text { ceptacles from a number of manufacturers and seems to appear } \\
\text { whether "jaw lock" or "ball lock" design fueling nozzles have been } \\
\text { used. Material builds up on the outer surface of the receptacle, } \\
\text { making it increasingly difficult to attach and properly seat the noz- } \\
\text { zle or to remove the nozzle after fueling. Improper seating of noz- } \\
\text { zles on these receptacles can lead to them popping off with signifi- } \\
\text { cant force when fueling is attempted. }\end{array}$ \\
\hline 03-4 & $4 / 27 / 03$ & $\begin{array}{l}\text { LACMTA experienced fueling breakaway separating on bus fueling } \\
\text { hose during operations with no undue tension on hose. High pres- } \\
\text { sure gas continued to flow through hose and caused hose to whip. } \\
\text { No injuries. By May a total of seven breakaway separations oc- } \\
\text { curred, all at Hanover stations. No injuries but minor PD reported. }\end{array}$ \\
\hline
\end{tabular}




\begin{tabular}{|c|c|c|}
\hline $03-5$ & $4 / 28 / 03$ & $\begin{array}{l}\text { Sherex } 5000 \text { bus receptacle failed at LACMTA. Internal brass part } \\
\text { broke into three pieces and clogged check valve, allowing gas to } \\
\text { escape once nozzle was removed. No injuries or PD. LACMTA } \\
\text { campaigned fleet and replaced this design with later Sherex design } \\
\text { using stainless steel for the part. }\end{array}$ \\
\hline $03-6$ & $9 / 3 / 03$ & $\begin{array}{l}\text { A Faber type } 1 \text { (all steel) CNG cylinder burst during fueling of a } \\
1994 \text { converted van in a Toronto suburb. "Massive external corro- } \\
\text { sion" is the apparent cause. Filling pressure was approximately } \\
1400 \text { psi at time of rupture. There is no evidence of the cylinder } \\
\text { having been inspected. There were no injuries but "extensive } \\
\text { damage to the vehicle and the natural gas dispensing system at } \\
\text { the station." }\end{array}$ \\
\hline 03-7 & $10 / 03$ & $\begin{array}{l}\text { CNG fuel line from tank behind rear axle in Ford E450 rubbing } \\
\text { against brake line. Wears through fuel line causing leak. Reported } \\
\text { by Laclede gas. Vehicles at Lambert Field (St. Louis airport). No } \\
\text { injuries, PD, fire, etc. }\end{array}$ \\
\hline 04-1 & $5 / 04$ & $\begin{array}{l}\text { Mack recall of up to } 317 \text { LNG trucks because of potential malfunc- } \\
\text { tion of Chart LNG tank pressure relief valves. Based on Chart } \\
\text { safety alert to all shrouded (truck) tank customers on replacing old } \\
\text { shroud doors with vented doors and installing pressure relief vent } \\
\text { tube. }\end{array}$ \\
\hline $04-2$ & Spring 04 & $\begin{array}{l}\text { CNG tanks on top of two WMATA (Washington, DC) CNG transit } \\
\text { buses were damaged when separate buses ran under low over- } \\
\text { passes. On first one cylinder scraped and gouged and one fuel } \\
\text { line severed. Internal tank solenoid valve stopped flow. On sec- } \\
\text { ond two PRDs were severed and gas vented down. Gas detection } \\
\text { system shut down system and isolated other cylinders. No injuries } \\
\text { or third-party property damage in either case. }\end{array}$ \\
\hline $04-3$ & $7 / 5 / 04$ & $\begin{array}{l}\text { Technician removed valve on CNG tank at Mesa, AZ Chevrolet } \\
\text { dealership without fully depressurizing it. Cylinder was } \\
\text { "launched... more than } 500 \text { feet" coming down through the roof of a } \\
\text { nearby body shop. Technician suffered minor injuries but body } \\
\text { shop employee was seriously injured. }\end{array}$ \\
\hline 04-4 & $10 / 04$ & $\begin{array}{l}63 \text { Converted Dallas police cars grounded because of leaky gas } \\
\text { lines and purported "CO" poisoning. In Sept } 26 \text { were "grounded for } \\
\text { the second time because of a faulty fuel hose in one car" where "a } \\
\text { pressurized fuel line had come unattached from the intake." Ford } \\
\text { Crown Vics converted by BAF. }\end{array}$ \\
\hline $05-1$ & $2 / 1 / 05$ & $\begin{array}{l}\text { Sacramento RT bus carrying students caught fire on I-5. Orion VIII } \\
\text { with Cummins } 8.3^{+} \text {gas engine, and original SCI tanks. Fire } \\
\text { started inside the bus, and did not involve the fuel system in any } \\
\text { way. No injuries. }\end{array}$ \\
\hline 05-2 & $2 / 05$ & $\begin{array}{l}\text { Montgomery County, MD's bus fleet received a slug of very wet } \\
\text { ( } 90-100 \mathrm{lbs} / \mathrm{mmscf} \text { gas (versus the } 20 \text { to } 30 \mathrm{lbs} \text { normal maximum). } \\
\text { This extremely unusual situation overwhelmed the gas dryer and } \\
\text { led to water in the bus fuel tanks, which then froze due to the } \\
\text { Joule-Thompson effect and cold weather, and disabled bus opera- } \\
\text { tion. }\end{array}$ \\
\hline
\end{tabular}




\begin{tabular}{|c|c|c|}
\hline $05-3$ & $2 / 28 / 05$ & $\begin{array}{l}\text { A severed gas main interrupted South County Area Transit's (Ven- } \\
\text { tura County, CA) normal supply of natural gas. Replacement gas } \\
\text { to the station from local off-shore production had a methane num- } \\
\text { ber much lower than that allowed for their Detroit Diesel engines, } \\
\text { making it likely they could be seriously damaged. Old diesel buses } \\
\text { were found and refurbished but full service was not restored for five } \\
\text { weeks until normal gas service was restored. }\end{array}$ \\
\hline $05-4$ & $6 / 05$ & $\begin{array}{l}\text { Two Xebec CNG fueling station dryer fires. No injuries in either } \\
\text { case. Both started in regeneration heater assembly. Poor mainte- } \\
\text { nance/operations practices identified by Xebec. }\end{array}$ \\
\hline $05-5$ & $7 / 26$ & $\begin{array}{l}\text { On July } 26 \text { five Waste Management CNG refuse trucks and one } \\
\text { sewage truck were destroyed by fire at Palm Desert, CA. Sus- } \\
\text { pected trash lodged in engine compartment or exhaust starting fire. } \\
\text { Believe PRDs relieved as designed. Believe trucks to be EcoTrans } \\
\text { converted Volvos. No injuries. }\end{array}$ \\
\hline $05-6$ & $8 / 3 / 05$ & $\begin{array}{l}\text { Four CNG trash trucks burned in Santa Monica, CA. Possible } \\
\text { cause an electrical short or failed high pressure regulator. Fire } \\
\text { started on truck not being fueled but spread to others, possibly be- } \\
\text { cause PRD's vented toward them. No injuries. }\end{array}$ \\
\hline $06-1$ & $2005-2006$ & $\begin{array}{l}\text { Thomas Built school buses in Virginia Beach and Charlottesville, } \\
\text { VA developed CNG leak just downstream of receptacle. No PD, } \\
\text { injuries nor fire. Repaired by Thomasbuilt. }\end{array}$ \\
\hline $06-2$ & $4 / 10 / 06$ & $\begin{array}{l}\text { Fire during decommissioning of CNG bus fueling station at NY City } \\
\text { MTA. Release of gas at service feed area ignited by unknown } \\
\text { source. No CNG buses involved. No injuries. }\end{array}$ \\
\hline $06-3$ & $4 / 28 / 06$ & $\begin{array}{l}\text { Toyota ran off road and hit CNG transit bus on roof. Shield over } \\
\text { CNG tanks damaged. No known damage to CNG fuel system. Se- } \\
\text { rious injuries to car driver, minor injuries to one bus passenger. }\end{array}$ \\
\hline $06-4$ & 10/7/06 & $\begin{array}{l}\text { Fire at rear of Kansas City airport shuttle bus caused by hydraulic } \\
\text { fluid leaking from engine fan onto exhaust pipe. PRD vented gas } \\
\text { safely. Three minor injuries, but an airport terminal was evacuated. }\end{array}$ \\
\hline $06-5$ & $12 / 7 / 06$ & $\begin{array}{l}2002 \text { CNG-powered Orion bus caught fire on I-85, a major com- } \\
\text { muter route outside Atlanta, during evening rush hour. No one was } \\
\text { hurt in the fire and the CNG tanks vented as designed. The fire } \\
\text { was caused by a hydraulic line failure. }\end{array}$ \\
\hline 07-1 & $1 / 11 / 07$ & $\begin{array}{l}\text { CNG powered trash truck caught fire in Irvine, CA. Fire originated } \\
\text { in trash. No noted involvement of CNG fuel system. No injuries, } \\
\$ 7000 \text { PD. }\end{array}$ \\
\hline $07-2$ & $2 / 7 / 07$ & $\begin{array}{l}\text { Gwinnett County (GA) Transit CNG Orion VII bus caught fire on I- } \\
85 \text { outside of Atlanta. No injuries. The fire was caused by a hy- } \\
\text { draulic line failure. No known CNG system involvement. }\end{array}$ \\
\hline $07-3$ & $3 / 22 / 07$ & $\begin{array}{l}\text { Boise, ID bus "burst into flames." No injuries. Looks like engine } \\
\text { fire as damage is to rear of bus. PRD venting system seems to } \\
\text { have worked as designed. No information on mfg, year, etc. yet. }\end{array}$ \\
\hline $07-4$ & $3-26-07$ & $\begin{array}{l}\text { Arsonist torched } 12 \text { vehicles in Seattle, WA city vehicle yard, in- } \\
\text { cluding one Honda CNG car. Fuel tank ruptured, apparently due to } \\
\text { localized flame impingement away from PRD. No injuries. }\end{array}$ \\
\hline $07-5$ & $5-26-07$ & Comdyne add-on tank at rear of Ford E350 SuperShuttle van rup- \\
\hline
\end{tabular}




\begin{tabular}{|c|c|c|}
\hline & & $\begin{array}{l}\text { tured during first fueling after repair of rear-end accident in Carson, } \\
\text { CA. One fatality. Stress corrosion cracking of overwrap caused by } \\
\text { exposure to battery acid in previous accident. }\end{array}$ \\
\hline $07-6$ & $7-07$ & $\begin{array}{l}\text { Flex line just downstream of receptacle tube sprung small leak. } \\
\text { Norwich, CT Public Utilities E350 Ford Van. }\end{array}$ \\
\hline $08-1$ & $2-12-08$ & $\begin{array}{l}\text { Santa Barbara County (CA) commuter bus (coach) fire in left rear } \\
\text { wheel well area. No involvement of CNG fuel system. }\end{array}$ \\
\hline $08-2$ & $4-22-08$ & $\begin{array}{l}1995 \text { or '96 privately-owned Dodge van Comdyne tank ruptured } \\
\text { during fueling in Grand Rapids, MI. Minor injuries to driver/fueler. } \\
\text { Probably caused by gouges/damage to fiberglass wrapping. Cylin- } \\
\text { ders had not been inspected in at least four years. }\end{array}$ \\
\hline $08-3$ & $3-25-08$ & $\begin{array}{l}\text { Explosion at Questar CNG fueling station in Salt Lake City, UT. Re- } \\
\text { lief valve fitting on one tank failed, causing failure of two other fit- } \\
\text { tings (banded together), which vented gas and caught fire. Fire- } \\
\text { fighters turned off gas and put out flames. No injuries. }\end{array}$ \\
\hline $08-4$ & $6-9-08$ & $\begin{array}{l}\text { Rupture of Lucas Type } 2 \text { cylinder on } 1998 \text { Ford E350 van owned } \\
\text { by Philadelphia Gas Works in Philadelphia suburb. Occurred soon } \\
\text { after fueling. No injuries. }\end{array}$ \\
\hline $08-5$ & $7-16-08$ & $\begin{array}{l}\text { Cylinder rupture on a } 1995 \text { Chrysler Corp. van in Corona, CA July } \\
\text { 16. Five people were hurt. }\end{array}$ \\
\hline $08-6$ & $9-19-08$ & $\begin{array}{l}\text { Out-of-service Los Angeles (LAMTA) bus broadsided by commuter } \\
\text { train. } 14 \text { train occupants suffered minor injuries. One roof- } \\
\text { mounted CNG cylinder broken loose and leaked. }\end{array}$ \\
\hline $08-7$ & $11-11-08$ & $\begin{array}{l}\text { During fueling NG leaked into passenger cabin of Chevrolet Subur- } \\
\text { ban, rendering four children unconscious (no permanent injury). } \\
\text { Conversion to NG had been done by owner of vehicle, father of } \\
\text { children. }\end{array}$ \\
\hline $08-8$ & $12-24-08$ & $\begin{array}{l}\text { Boston MBTA bus struck object, ripped off cylinder cover on roof } \\
\text { and compromised CNG fuel line. Firefighters let gas dissipate, } \\
\text { then transit employee shut off valve. No injuries. }\end{array}$ \\
\hline $08-9$ & $12-29-08$ & $\begin{array}{l}\text { Phoenix, AZ LNG transit bus fire started at rear wheel oil seal. No } \\
\text { injuries or explosion. Bus was allowed to burn to the ground. }\end{array}$ \\
\hline
\end{tabular}

The Clean Vehicle Education Foundation makes a great effort to provide secure, accurate and complete information regarding NGV incidents. However, portions of the information contained in this document may be incorrect or not current. Any errors or omissions should be reported to jlapetz@cleanvehicle.org for investigation.

The Clean Vehicle Education Foundation, it's officers, employees or agents shall not be liable for damages or losses of any kind arising out of or in connection with the use or performance of the information provided herein, including but not limited to, damages or losses caused by reliance upon the accuracy or timeliness of any such information, or damages incurred from the viewing, distributing, or copying of those materials.

The information provided in this document is provided "as is." No warranty of any kind, implied, expressed, or statutory, including but not limited to the warranties of noninfringement of third party rights, title, merchantability, or fitness for a particular purpose, is given with respect to the contents of this document. 
Clean Vehicle Education Foundation - 6011 Fords Lake Ct., Acworth, GA 30101-8469 - www.cleanvehicle.org 


\section{Appendix L - Detailed File of NGV Incidents}

In order to meet NETL's requirements that the final report accompanying form 241.3 be uploaded as one document in pdf file, this appendix would be unacceptably compromised (all files in the appendix files are listed by Adobe in alphabetical order, rather than under appropriate subfolders). Therefore this appendix has not been included.

Please contact CVEF for a CD copy of the Detailed File of NGV Incidents. 


\section{Recommended Minimum In-Service Inspection of CNG Station Storage Cylinders and Pressure Vessels}

J. B. Dimmick December 30, 2008

\section{Introduction:}

Pressure containers used in filling stations for compressed natural gas vehicles commonly include various ASME Code ${ }^{1}$ pressure vessels or gas cylinders that are commonly used for shipment of gases or as vehicle fuel containers. The system standard for CNG stations, NFPA 52 Vehicular Fuel Systems Code ${ }^{2}$, 8.16.1 states: "Containers and their appurtenances, piping systems compression equipment, controls, and detection devices shall be maintained in safe operating condition and according to manufacturers' instructions." However, in many cases there are no manufacturer's instructions regarding pressure vessels. The discussion explains the source for the recommendations and how they were developed from generally accepted practices or standards.

\section{Purpose:}

The purpose of this document is to provide specific recommendations for inspection or retest of containers and attached gages and pressure relief devices (PRDs) whenever inspection is not regulated by an Authority Having Jurisdiction (AHJ). These recommendations are based on the usual recommended practices in standards and regulations.

\section{ASME Code Pressure Vessels:}

3.1. NFPA 522006 defines a pressure vessel as a container or other component designed in accordance with the ASME Boiler and Pressure Vessel Code or the CSA B51-03, Boiler, Pressure Vessel and Pressure Piping Code. The "other components" may include but are not limited to blow down vessels, filter housings, dryer housings, heat exchangers and meter housings. Any ASME Code component will be marked with the U stamp authorized by ASME.

3.2. The ASME Code is commonly used in the US, but the scope of the ASME Code does not include any requirements for in-service inspection. A separate inspection code, ANSI/NB-23, National Board Inspection Code ${ }^{4}$, contains general and specific rules for such in-service inspections. In general, there is a lack of uniformity in the way that various federal, state or local authorities use, omit, or modify the ANSI/NB-23 rules. Most states have an agency that is responsible to regulate pressure vessel safety, often including $\mathrm{CNG}$ vessels. The National Board of Boiler and Pressure Vessel Inspectors publishes ANSI/NB-23 and also maintains documents on their website with synopses of state, provincial and local pressure vessel regulations. The site also gives contact information for the AHJ in each of the different jurisdictions. 
Follow this link to obtain a copy of ANSI NB-23 or to access the synopses. https://www.nationalboard.org/nationalboard/Default.aspx For the synopses, at the left side, select E-Publications and Directories. Select the last menu item, National Board Synopsis of Boiler and Pressure Vessel Laws, Rules and Regulations. You must register for a password in order to access the synopses, but there is no charge. Each jurisdiction synopsis also includes the contact information for the authority having jurisdiction over pressure vessels and boilers.

3.3. Some states may delegate CNG vessel safety to state or local fire officials. The fact that the US regulatory environment leaves many locations without a clear legal rule for in-service inspection of $\mathrm{CNG}$ vessels does not mean that such inspections are unnecessary, only that they are not a proactive legal requirement.

\section{Gas Cylinders:}

In some stations, gas cylinders not pressure vessels are used for cascade storage or buffer containers. These cylinders are more commonly intended to be used in shipping compressed gases such as oxygen or nitrogen or $\mathrm{CNG}$, or as $\mathrm{CNG}$ vehicle fuel containers. Station designers have found it convenient to use these cylinders as cascades even though this use is outside the scope of the original standards regulations.

Cylinders are defined in NFPA 52 as CNG containers constructed, inspected and maintained in accordance with DOT and Transport Canada (TC) regulations or ANSI $\mathrm{NGV}_{2}{ }^{5}$. NFPA $52 \S 4.4 .4$ requires that these cylinders be inspected, equipped, maintained and retested in accordance with the DOT or TC regulations or ANSI NGV2. NFPA therefore accepts the use of these specifications of cylinders outside the limited scope of their standards of construction. Since the periodic inspection or retest requirements are different depending on the standard of construction, the general class of "cylinders" is subdivided as described below.

\subsection{Compressed Gas Cylinders:}

In this document, these are DOT or TC cylinders originally intended as containers for the transportation of compressed gases as specified by the DOT Pipeline and Hazardous Materials Safety Administration (PHMSA), typically DOT-3AA ${ }^{6}$ specification cylinders, or vehicle fuel containers under the National Highway Transportation Safety Administration (NHTSA). TC cylinders are intended as containers for the transportation of compressed gases under the Transportation of Dangerous Goods (TDG) section of TC. Fixed pressure vessels and cascades are therefore outside the scope of TC TDG or DOT PHMSA regulations, but using such cylinders on CNG filling stations is a long-established practice and is acceptable to NFPA as well as many jurisdictions.

It is believed that although the actual use of these cylinders in stations is outside the scope of the respective federal regulations, NFPA intends that the same in-service inspection or retest requirements apply as if the cylinders were in the federally 
regulated service. OSHA has issued an interpretation to this effect concerning required periodic retesting of DOT PHMSA DOT-3AA cylinders in CNG filling station service. The OSHA interpretation can be found at the end of this document.

\subsection{Vehicle Fuel Containers:}

In this document, these cylinders are ANSI NGV2 or FMVSS $304^{7}$ vehicle fuel containers under the National Highway Transportation Safety Administration (NHTSA). Their use on stations is outside the scope of their original standards, but they are permitted and used in stations in accordance with NFPA 52.

The OSHA interpretation does not apply explicitly to either NGV2 or FMVSS 304 vehicle fuel containers used as cylinders in a CNG station. NGV2 and FMVSS 304 vehicle cylinders are intended to be visually inspected at least every three years. Applying the rationale in NFPA 52 and extrapolating from the OSHA interpretation, three years would be the maximum inspection interval for these cylinders in station use.

\section{Authorities Having Jurisdiction (AHJ):}

NFPA 52 defines an AHJ as "...responsible for enforcing the requirements of a code or standard, or for approving equipment, materials, an installation or a procedure." AHJs include various state, local and Canadian provincial safety agencies responsible for pressure vessels and equipment but may also include fire marshals and OSHA. Wherever an $\mathrm{AHJ}$ requires inspection of $\mathrm{CNG}$ vessels, cylinders or appurtenances such as relief valves, these should be considered minimum requirements. Since not all locations are regulated by an $\mathrm{AHJ}$ for $\mathrm{CNG}$, and because AHJs may or may not impose specific requirements for $\mathrm{CNG}$ vessels, it may be necessary to determine an effective inspection program without resort to regulators.

The CSA B51 Code is administered in Canada by the various provincial pressure vessel safety authorities who also establish the requirements for in-service inspections. The CSA B51 Code includes both pressure vessels and cylinders at CNG stations. The requirements for retest or requalification depend on the standard used to register the design of the cylinders or vessels.

Where any code or standard may conflict with the requirements of an AHJ, the AHJ requirements take precedence. The AHJ may regulate the inspection and retest of both ASME Code vessels and cylinders that are used in CNG stations.

\section{Underwriter's Requirements for Inspection:}

In some cases where the AHJ does not require inspections of vessels, the insurance company that underwrites coverage for the station may require periodic inspection of vessels. This will often be carried out in accordance with the National Board Inspection Code (ANSI NB-23). 


\section{Recommended In-Service Inspection of ASME Pressure Vessels When There Are No State or Local Regulations in Effect:}

If the AHJ does not impose requirements and in the absence of explicit ASME vessel inspection requirements in NFPA 52, we suggest that the recommended basis for the inspection of ASME pressure vessels be ANSI/NB-23. The purpose of ANSI/NB-23 is given in the introduction as: "...to maintain the integrity of pressure-retaining items after they have been placed in service by providing rules for inspection, installation, repair and alteration, thereby ensuring that these objects continue to be safely used. ...and is intended to provide guidance to jurisdictional inspectors, users, and organizations performing repairs and alterations..." "Where complete details are not included in this Code, the Code user is advised to seek technical guidance."

As is normal for codes, in any "conflict with a jurisdictional regulation, the jurisdictional regulation shall govern." Some specific requirements from the 2006 addenda of ANSI/NB-23 have been identified as follows.

\subsection{ANSI/NB-23 Provisions for Inspection Interval:}

The following provisions of ANSI/NB-23 have been selected as providing guidance in determining inspection frequency.

7.1.1. RB-9040 contains a list of the information that may be used in determining inspection frequency. The relevant deterioration mechanisms are used to determine an inspection frequency. Eliminating special causes such as fire or mechanical damage, the dominant mechanisms for CNG vessels are corrosion and fatigue.

7.1.2. RB-9050 requires a basic interval for "internal inspection or complete inservice evaluation" of "ten years or one half the remaining life, whichever is less." It also allows longer intervals based on technical justification. The ASME Section VIII Division 1 vessels that are common in CNG stations are not designed to a finite life. Vessels known to have developing flaws and vessels designed for a specific life under Divisions 2 or 3 may be subject to the one half remaining life requirement.

7.1.3. RB-9110 (e) requires that "All pressure vessels above ground be given an external examination after operating the lesser of five years, or one quarter of the remaining life, preferably while in operation.” There is a basic assumption that such a vessel is subject to external corrosion. As in the case of RB-9050, Division 1 vessels typically are not designed for a specific life and the five year interval would apply. RB-9040 also contains comprehensive guidance for vessels subject to internal corrosion, but this may not be an issue for CNG vessels. 
7.1.4. The natural gas vehicle industry has established limits on the permissable levels of contaminants to effectively prevent corrosion of fuel containers including pressure vessels. These limits are given in NFPA 52 §4.2, Composition. ANSI/NB-23 RB-9120 (a) allows that the gas be considered non-corrosive if "The non-corrosive character of the content, including the effect of trace elements has been established by at least five years comparable service experience with the fluid being handled." General experience in the CNG industry indicates that CNG may be considered noncorrosive.

\subsubsection{RB-9260, Evaluating Exposure of Pressure Retaining Items to Cyclic} Fatigue, refers to the data listed in RB-9040 to be used in determining the "allowable number of cycles." A definitive fatigue analysis can be very involved, but is seldom required except in extraordinary circumstances. Cyclic fatigue may not be an issue for CNG vessels based on the absence of any indications of fatigue failure history and the common vessel designs used for CNG. Most high-pressure CNG vessels used in buffer and cascade systems are designed to the requirements of ASME Section VIII, Division 1. This Division does not explicitly require a fatigue analysis for design. In addition, these vessels are generally designed as seamless forgings without significant fatigue stress raisers and of "streamlined" shape as illustrated in Appendix 22 of Division 1.

7.1.6. For $\mathrm{CNG}$ vessels designed to Divisions 2 or 3 , a design fatigue analysis may have been required and may form the basis for establishing inspection intervals with a detailed fatigue analysis.

\subsection{Recommended Default Vessel Inspection Interval:}

The following inspection intervals are recommended in the absence of any specific regulations by the AHJ.

\subsubsection{Division 1 Vessels Operating in Accordance with NFPA 52:}

For operating conditions complying with NFPA 52 \$4.2 Composition, this document recommends an initial inspection interval for ASME Code Section VIII $\mathrm{CNG}$ vessels is five years for external inspection with no required internal inspection. This is consistent with RB-9110(e), above. We suggest that this represents good practice given the service conditions of $\mathrm{CNG}$ station pressure vessels.

7.2.2. Division 2 or 3 Vessels Operating in Accordance with NFPA 52:

For ASME Code Section VIII Division 2 or 3 vessels operating under the NFPA 52 conditions, a fatigue analysis may be required to determine an inspection 
interval for this damage mechanism. It is recommended that the vessel manufacturer be contacted for specific recommendations.

\subsubsection{Vessels Not Operating in Accordance with NFPA 52:}

For operating conditions outside those specified in NFPA 52, the inspection interval should be determined after a detailed technical evaluation in accordance with RB-9110.

\section{Inspection of Pressure Gages:}

RB-6251, Gages, requires that pressure gages be compared to others on the system for accuracy and that they be correctly connected to indicate the actual pressure in the vessel. "When required, the accuracy of pressure gages should be verified by comparing the readings with a standard test gage or a dead weight tester." This is the recommended practice for all pressure measuring instruments, not just mechanical gages, on stations.

\section{Pressure Relief Devices (relief valves or rupture disks):}

9.1. NFPA $52 \S 4.5 .2 .3$ requires that "Pressure relief valves protecting ASME pressure vessels shall be repaired, adjusted and tested in accordance with NB-23, National Board Inspection Code."

9.2. NFPA $52 \S 8.10 .2$ states "pressure relief valves shall be tested at least every three years."

The NFPA requirements are seen as two minimum requirements with the three year interval taking effect if the relief valve is not protecting an ASME pressure vessel or if NB-23 would allow a longer interval. As in the case of vessel inspections, the AHJ can require greater or lesser requirements and takes precedence over the standards. NB-23 provides the following guidance.

9.3. RB-6252 refers to RB- 8000 for the inspection of pressure relief valves and rupture disks.

9.4. RB-6253, Control Devices, requires inspection either of actual operation or the procedures and records for verification of operation.

ANSI/NB-23, RB-8000 contains rules for inspection of Pressure Relief Devices (PRDs).

The function of the PRD on these vessels should not be confused with the function of a PRD on a CNG vehicle fuel container. The PRD on the vehicle fuel container is intended only to activate during a fire and prevent container rupture from fire exposure. It does not provide protection against over pressurization from any other 
source. ASME vessels are required to be protected by pressure activated PRDs that will protect against overpressurization from any source.

\subsection{RB-8010 defines the scope as follows.}

"The most important appurtenances on any pressurized system are the pressure relief devices provided for overpressure protection of that system. These devices such as safety valves, safety relief valves, pilot valves, and rupture disks or other nonreclosing devices that are called upon to operate and reduce an overpressure condition.

"These devices are not designed or intended to control the pressure in the system during normal operation. Instead, they are intended to function when normal operating controls fail or abnormal system conditions are encountered.

"Periodic inspection and maintenance of these important safety devices is critical to ensure their continued functioning and to provide assurance that they will be available when called upon to operate."

Note the priority as the "most important appurtenance." This recognizes that control systems may fault or fail, but the vessel should be protected with the PRD system.

Note also that the PRD is not intended to have any role in the normal operation of the system, being wholly a redundant backup for all of the other pressure controls. This also indirectly but clearly defines any operation of the PRD as abnormal and resulting from failure or faults in the other pressure control systems.

The third important scope issue is the need for periodic inspection and maintenance. This is normally expected for any such device operating under a gage or measuring system quality assurance program. Fueling systems for vehicles are sometimes subject to local or state authorities, but these may not require this periodic inspection and maintenance of all PRDs in the system.

9.6. Operating a PRD system within this scope requires the following:

9.6.1. The PRD system must be entirely independent of the normal operating pressure control. There must be no identifiable fault in the normal operating pressure control system that could interfere with the ability of the PRD to prevent or reduce to a safe level any overpressure in the vessel system.

9.6.2. Any actual operation of the PRD must be treated as an operating pressure system failure or fault. Since overpressure is the principal immediate hazard for any pressure system or vessel, such a fault or failure must result in corrective action. Simply venting sufficient pressure so that the PRD reseats or replacing a rupture disk or non-reclosing PRD is not sufficient. This point is critically important for safe operation. 


\section{Inspection or Retest of Pressure Relief Devices:}

The periodic inspection and maintenance system for the PRD is as important as the initial presence of a PRD in the system. PRDs, particularly those of the reclosing type common on flammable gas systems, are mechanically complex and have a variety of deterioration and failure mechanisms. Simple visual inspection is not sufficient for these valves and periodic pressure testing is also required by RB-8400. While many PRDs on steam, air or similar systems have lifting devices for an on-system function check, codes such as NFPA 52 \$4.5.2.2 prohibit lifting devices on PRDs for flammable fuel gases.

RB-8410 gives guidance in determining and adjusting the interval for periodic inspection and maintenance of relief valves. The intervals are dependent on both the system and fluid details as well as the results of each inspection. It is also clear that compliance with these requirements will require the maintenance of spare PRDs to be substituted while periodic maintenance or repair is performed. "If valves are found to be defective or damaged by system contents during inspection, the inspection interval should be shortened until acceptable inspection results are obtained." Where detailed records are not available, our recommended initial inspection frequency for PRDs in clean dry gas service is three years. This appears appropriate under NB-23 for operating conditions as specified in NFPA 52 and also is consistent with NFPA 52 \$8.10.2 and also with Canadian Codes CSA B 51 and CSA B $109^{8}$. If the PRD is found to be in unsatisfactory condition, the interval to the next inspection should be shortened.

\section{Pressure Vessel Inspector Qualifications:}

The recognized qualification for inspectors of pressure vessels is licensing by the National Board of Boiler and Pressure Vessel Inspectors. A list of organizations providing such inspections is available from the National Board of Boiler and Pressure Vessel Inspectors.

In certain cases, owner-operators of vessels with sufficient technical expertise may also be considered qualified, subject to the AHJ. Owner-operator inspectors must be qualified and are generally employed in large plants. The AHJ will audit the owner-operator inspectors for compliance.

In some jurisdictions such as California and Pennsylvania, the AHJ will directly provide inspection services by qualified inspectors.

\section{Qualifications for Calibration and Repair of Pressure Relief Valves:}

ANSI/NB-23 Part RE, Repairs of Pressure Relief Valves, should be applied to all calibration and repair. The basic requirement is that the repair organization holds a National Board "VR" Certificate of Authorization. A list of "VR" certificate holders is available from the National Board of Boiler and Pressure Vessel Inspectors. 


\section{Recommended In-Service Retest and Inspection of Cylinders Installed in CNG Filling Stations:}

\subsection{DOT or TC Compressed Gas Cylinders:}

In accordance with the requirements of NFPA 52 and the OSHA interpretation (see below), DOT and TC compressed gas cylinders should be subjected to in-service retesting and inspection in general accordance with the controlling specification or Special Permits. In Canada, the provincial pressure vessel authority should be contacted for guidance on inspection of cylinders.

Based on the interpretation received from OSHA, we believe DOT PHMSA and TC TDG compressed gas cylinders, either specification cylinders such as DOT-3AA or Special Permit cylinders should be inspected and retested by qualified retesters and at the intervals as required in the DOT or TC regulations. The typical retest interval for specification cylinders containing natural gas is five years with three years being more typical for Special Permits. Prior to 2006 DOT PHMSA used the term "Exemption" instead of "Special Permit." Special Permits also often specify a maximum life after which the cylinders must be removed from service. Users of Special Permit cylinders should obtain and retain copies from the issuing agency, either DOT PHMSA or TC TDG. The basic specifications will require a hydrostatic pressure test as well as both internal and external examinations. DOT has also issued Special Permits that allow the use of external ultrasonic examination as a substitute for the pressure test and internal inspection. This newer method may be preferable where available.

Some station operators may not be under the jurisdiction of OSHA. Cylinders in these stations must still be periodically inspected or retested to assure they are safe for continued use. In the absence of an AHJ, these station operators should still abide by the OSHA interpretation and NFPA intent for cylinders.

\subsection{Qualifications for Retesting and Inspection of Compressed Gas Cylinders:}

DOT PHMSA and TC TDG maintain listings of authorized retest facilities. All periodic retest and inspection in accordance with DOT PHMSA or TC TDG regulations must be performed by one of these authorized retest facilities.

\subsection{ANSI NGV2 or FMVSS 304 CNG Fuel Containers:}

DOT NHTSA FMVSS 304 and ANSI NGV2 CNG fuel containers in vehicles must be inspected externally at least every three years in accordance with the manufacturer's guidelines and CGA C-6.4 $4^{9}$ and have a limited lifetime after which they must be removed from service. Since use on a fuel station is outside the specific scope of use for these cylinders, if they are used in a station application, the inspection interval and guidelines should be in accordance with the cylinder 
manufacturer's recommendations for use in a station, but not less than the three year interval.

\subsection{Qualifications for Inspection of CNG Fuel Containers:}

The qualifications for inspectors are set out in CGA C-6.4. CSA America also maintains a roster of $\mathrm{CNG}$ container inspectors certified by their test program. CVEF can provide a list of trainers for CGA C-6.4.

\section{PRDs for Cylinders Installed in CNG Filling Stations:}

Although cylinders are used outside the scope of their original manufacturing specification when installed in stations, they should be equipped with PRDs as required in their original specifications.

14.1. NFPA $52 \S 4.4 .4$ requires that cylinders be “... equipped... in accordance with..." DOT, TC or CSA standards or special permits. These standards and special permits all have specific requirements for PRDs.

14.2. NFPA 52 \$4.5.1 requires that cylinders be equipped with PRDs in accordance with several different standards. Taken in combination with 4.4.4, the PRDs used on cylinders should be those required by their various standards of construction and use.

14.3. These PRDs are intended to protect against rupture in a fire, but do not protect against overpressurization due to a failure in the fill station controls. Because the materials of construction and operating stresses in cylinders may be significantly different from ASME Code vessels, the ASME Code pressure relief valve rules may not protect cylinders from rupture in a fire. NFPA $52 \$ 4.5 .2$ requires both cylinders and pressure vessels to be protected spring-loaded pressure relief valves in accordance with the ASME Boiler and Pressure Vessel Code.

14.4. A pressure relief valve in addition to the standard cylinder PRDs is required to protect each bank of the buffer or cascade from accidental over pressurization by the compressor. In this regard, the application of these cylinders to a filling station is similar to the application of ASME pressure vessels and a similar provision for pressure relief in the event of a pressure control system failure or fault is recommended. ASME Code pressure relief valves must be installed with set pressure and capacity to prevent the station from overpressurizing the cylinders. These pressure relief valves should then be maintained and inspected as described above and as required for pressure vessel relief valves in ANSI/NB-23.

15. Websites of Interest: 
www.cvef.org The Clean Vehicle Education Foundation

www.nfpa.org The National Fire Protection Association

www.nationalboard.org The National Board of Boiler and Pressure Vessel Inspectors www.osha.gov the Occupational Safety and Health Administration http://hazmat.dot.gov/sp app/special permits/exe 0000.htm US DOT site for downloading the most current special permits and exemptions by number. The DOT retest and inspection requirements are included in the exemption or special permit. www.csa-intl.org The CSA International website for CNG standards including ANSI/CSA NGV2.

www.cganet.com Compressed Gas Association website for purchase of CGA C-6.4

\section{OSHA Interpretation Regarding DOT Compressed Gas Cylinders Used In Stations:}

\section{U.S. Department of Labor \\ Occupational Safety and Health Administration Washington, D C. 20210 \\ Reply to the Attention of: DCP/GICA/MIMID-1 13}

SEP 262002

Hank Seiff, P.E.

Director of Technology

The Natural Gas Vehicle Coalition

400 North Capitol Street, NW

Washington, DC 20001

Dear Mr. Seiff:

Thank you for your November 9, 2001 letter to the Occupational Safety and Health Administration's (OSHA's) Directorate of Compliance Programs (DCP). You have questions regarding OSHA's Compressed Gases (General Requirements) Standard, $\S 1910.101$. Please be

aware that this response may not be applicable to any question or situation not delineated within

your original correspondence. Your specific questions are related to inspection and maintenance requirements for compressed natural gas (CNG) cylinders at vehicular fueling stations and the application of a latter version of a document which is incorporated by reference into OSHA 
standards.

Scenario: U.S. Department of Transportation (DOT) 3AA cylinders are used for storage of

high pressure gas at some $\mathrm{CNG}$ vehicular refueling stations.

Question: Does OSHA have any requirements or guidelines for station operators on the

inspection and maintenance of these fuel storage cylinders?

Reply: First, OSHA would have jurisdiction over the situation you describe. The DOT 3AA cylinders are not involved in the movement of materials involved with interstate commerce; the cylinders are used merely for on-site storage of CNG. Since DOT has no authority to enforce its regulations over the storage of $\mathrm{CNG}$ in cylinders at the described locations, there is no preemption of OSHA authority and OSHA would enforce its workplace health and safety regulations over the situation you describe.

\section{OSHA/DOT Requirements}

While OSHA has not published any guidelines on the situation you describe, we do have regulations related to the inspection and maintenance of compressed gas cylinders, which include

CNG cylinders. OSHA has incorporated by reference DOT Hazardous Materials Regulations, 49

CFR Parts 171-179 (amended January 1, 1970).

Part of OSHA regulation $\$ 1910.101(a)^{1}$ requires station operators/employers to determine that compressed gas cylinders under their control are in a safe condition to the extent that the cylinders' mechanical integrity can be determined by visual inspection. Additionally, OSHA and DOT recognize that, based on the service of the cylinder, i.e. material, pressure, etc., a visual inspection alone may be insufficient to determine the mechanical integrity of a compressed gas cylinder.

Since employers may not be able to adequately determine the cylinder condition based merely on a visual inspection, OSHA and DOT require visual and other inspections as prescribed in the referenced DOT regulations. DOT has a regulation, 49 CFR 173.34, Qualification, Maintenance and Use of Cylinders (a copy of this section of the 1970 incorporated standard is attached), which is applicable to your question.

In particular, 49 CFR 173.34(e), Periodic retesting and reinspection of cylinders, requires retesting of DOT 3AA cylinders every five years. This requirement would be applicable to DOT 3AA cylinders containing CNG. Further, 49 CFR 173.34(e)(1) specifies criteria to be included in a periodic retest which includes a visual internal and external examination together with a test by interior hydrostatic pressure in a water jacket or other apparatus of suitable form. 
Please be advised that in this case, the exceptions found in the OSHA-incorporated standard, 49 CFR 173.34(e)(10), are not applicable to CNG cylinders because your CNG cylinders do not meet the listed service criteria ("...listed in the table below and used exclusively in the service indicated [emphasis added]..."). The table in 49 CFR 173 $.34(e)(10)$ lists only the services of liquefied petroleum gas, anhydrous ammonia, fluorinated hydrocarbons, butadiene, and liquefied hydrocarbon gas; $\mathrm{CNG}$ is not one of these specified service materials.

Question \#2: What is OSHA's position on whether an employer may follow later versions of the Compressed Gas Associations (CGA) pamphlets?

Response: Employers may use the current revision of a national consensus standard relative to a previous revision which was incorporated, usually with changes, into OSHA regulations. This, however, is predicated on compliance with a current national consensus standard which provides at least the same level of safety and health protection as would otherwise be provided by complying with the previous national consensus standard adopted into 051-IA regulations. Therefore, OSHA recommends that employers comply with later versions of CGA pamphlets which provide at least the same level of safety and health protection as would otherwise be

29 CFR 19 10.101(a) Inspection of compressed gas cylinders. Each employer shall determine that compressed gas cylinders under his control are in a safe condition to the extent that this can be determined by visual inspection. Visual and other inspections shall be conducted as prescribed in the Hazardous Materials Regulations of the Department of Transportation (49 CFR parts 1 71-1 79 and 14 CFR part 103). Where those regulations are not applicable, visual and other inspections shall be conducted in accordance with Compressed Gas Association Pamphlets C-6-1968 and C-8-1962, which is incorporated by reference as specified in Sec. 1910.6.

provided by complying with previous CGA pamphlets that have been incorporated by reference into OSHA standards.

Thank you for your interest in occupational safety and health. We hope you find this information helpful. OSHA requirements are set by statute, standards and regulations. Our interpretation letters explain these requirements and how they apply to particular circumstances, but they cannot create additional employer obligations. This letter constitutes OSHA's interpretation of the requirements discussed. Note that our enforcement guidance may be affected by changes to OSHA rules. Also, from time to time we update our guidance in response to new information.

To keep apprised of such developments, you can consult OSHA's website at http://www.osha.gov. If you have any further questions, please feel free to contact the Office of General Industry Enforcement at (202) 693-1850. 
Sincerely,

\title{
$|\mathbf{s}|$
}

\author{
Richard E. Fairfax, Director \\ Directorate of Enforcement Programs
}

Attachment: 49 CFR 173.34, Qualification, Maintenance and Use of Cylinders, 1970

\footnotetext{
${ }^{1}$ ASME Boiler \& Pressure Vessel Code, The American Society of Mechanical Engineers, New York, NY

${ }^{2}$ NFPA 52 Vehicular Fuel Systems Code 2006 Edition, The National Fire Protection Association, 1 Batterymarch Park, Quincy, MA

${ }^{3}$ CSA B51-03 Boiler Pressure Vessel, and Piping Code, Canadian Standards Association, Mississauga, ON, Canada

${ }^{4}$ ANSI/NB-23, NBIC National Board Inspection Code, 2006 Addendum, The National Board of Boiler and Pressure Vessel Inspectors, Columbus, $\mathrm{OH}$

${ }^{5}$ ANSI NGV2-3007, American National Standard for Compressed Natural Gas Vehicle Fuel Containers, CSA America, Inc., Cleveland, $\mathrm{OH}$

${ }^{6}$ 49CFR 178.37 , Specification $3 A A$ and $3 A A X$ seamless steel cylinders, Title 49, US Code of Federal regulations, Office of the Federal Register, National Archives and Records Administration, Washington, $\mathrm{DC}$

${ }^{7}$ 49CFR 5571.304 , Standard No. 304; Compressed natural gas fuel container integrity, Title 49, US Code of Federal regulations, Office of the Federal Register, National Archives and Records Administration, Washington, DC

${ }^{8}$ CSA B109, Natural Gas for Vehicles Installation Code, August 2000, CSA International, Toronto, ON, Canada

${ }^{9}$ CGA C-6.4-2003, Methods for Visual Inspection of Natural Gas Vehicle (NGV) Fuel Containers and their Installations, Compressed Gas Association, Inc., Chantilly, VA
} 


\section{Appendix N - Recertification Results from Surveys}

Fleet

Capital City Coach, Indianapolis, IN Carlos Garcia, Ontario, CA

Citizens Gas and Coke, Indianapolis, IN City of Asheville, NC

City of Kingwood, MO

City of Long Beach, CA Gas Dept

City of Merced, CA

City of Tulare, CA

City of Visilia, CA

Fiba Canning, Scarborough, ON, Canada

Greater Portland Transit, Portland, ME Greenes Auto Service, Indianapolis, IN Hoffman Beverage, Virginia Beach, VA Lansing Community College, Lansign, M

Manhattan Beer, NYC, NY

Monterey-Salinas Transit, Monterey, CA

Montgomery County, Maryland

MV Transit, Fremont, CA

Oklahoma Natural Gas, Davenport, OK

Omnitrans, San Bernardino, CA
6. Does the fact that most CNG cylinders have a design life of 15 years (and should be replaced after that time) cause a problem for your fleet?

Yes

\section{No Additional Comments}

1 Fleet replacement criteria

is less than 15 years

1 Under normal circumstances we will replace vehicle before life of clyinder Ther is no know reson that these Cylinders need to be replaced at 15 years This needs to be reviwed by DOT.

1 Most of our vehicles are replaced before the 15 year life cycle of the cylinders.

1 Replacement schedule for fleet equpiment is generally ten years or less.

We see no reason why these can't be retested.

1

7. If "Yes," would it be useful to have a method of "recertifying"

cylinders so that they could be used

for an additional period of time?

\section{$\underline{\text { Yes }}$ \\ No Additional Comments}

There will be concerns of running buses longer. The FTA mandates that transit operators that use federal money must use the vehicle for 12 years or 500,000 miles. When money is tight, transit operators will run vehicles longer. In this case it presents another consideration to there is not too much maintainance places, and is too expensive

Yes to recertifying the clyinders,

if there good keep using them

This would have to be a in shop prosses

Absolutely!

Cylinders are in truck and behind back seat and not subject to damage. Vehicles used for training only 
Omnitrans, San Bernardino, CA 2

QC Metrolink, Rock Island, IL

Questar, Salt Lake City, UT

Sacramento RTD, Sacramento, CA

Santa Clarita, CA Transit

SunLine Transit, Thousand Palms, CA

Southwest Transportation Agency, Caruthers CA

Valley Transit, Walla Walla, WA

Veolia Transit, Chula Vista, CA

Veolia Transportation, Springfield, VA

West Wind Farms, Deer Lodge, TN

TOTALS:

operate transit buses past there normal life.

Yes and No, the normal life cycle of a Transit bus is supposed to be 12 years.

Unfortunately they do not necessarily get replaced in 15 years.

Vehicles still have useful life but must be replaced due to age of tanks and the cost of replacement.

The FTA guidelines for vehicle life of transit buses in 10-12 years or 500,000 miles this will be well within the 15 year limit.

The cost of replacing the tanks is more then a school would want to put in a 15 year old bus. But would not have a choose at this time, because a new bus is much more.

1

Even though transit buses are designed to have a life of 12 years they are usually kept around much longer.

It will be a financial hindrance to

continued use of the CNG fuel option when the existing tanks expire.
The cylinders are still passing inspections and do not appear to need relacement. It is our belief the life expectancy should be longer than 15 years.

If the tank would last as long as the bus was still safe to run $\&$ not cost to much to keep on the road. 


\section{Appendix D (informative) to NGV2 \\ Procedure for Requalifying All-Steel Natural Gas Vehicle Storage Cylinders}

Note: This informative Appendix has been written in normative language to facilitate its adoption where users of the Standard or regulatory authorities wish to adopt it formally as additional requirements to this Standard.

\section{D.1 Introduction}

This Appendix establishes the minimum requirements for requalifying cylinders that are designed in accordance with this Standard and have reached the end of the service period specified by their manufacturers.

The design life of an all-metal steel cylinder, which is based on the rate of fatigue crack growth in the cylinder wall is determined by a variety of factors, including material properties, the number of pressure cycles, the wall stress associated with the pressure cycle amplitude, and the nature of the crack-initiating feature on the cylinder.

\section{D.2 Cylinder Types and Service Conditions}

\section{D.2.1 Cylinder Types Covered}

The types of cylinders covered in this Appendix are;

a) steel vehicle cylinders that

i) are designed for use as on-board fuel storage containers for vehicles powered by natural gas;

ii) meet the requirements of this Standard

\section{D.2.2 Service Conditions}

Section 2 of this Standard specifies the service conditions for cylinders that are used for the onboard storage of natural gas as a fuel for automotive vehicles.

\section{D.3 Visual Inspection Procedure}

\section{D.3.1 Original Markings}

The inspector (the person responsible for requalifying the cylinders) shall ensure that the original markings on the cylinder are clearly visible and unambiguous. If the markings do not include an identification of the manufacturer, the cylinder serial number, and the design registration number, the cylinder shall not be requalified under this procedure.

\section{D.3.2 Visual Damage}

Prior to ultrasonic inspection, the cylinder shall be visually inspected in accordance with CGA C6.4 for heat damage, dents, gouges, or severe external corrosion. Severe corrosion is metal loss that causes a local reduction in the cylinder wall thickness of more than $5 \%$ of the original or minimum design wall thickness. A cylinder that fails the visual inspection shall be destroyed. 
(C) Canadian Standards Association

D.3.3 Cleaning

To ensure the accuracy of the ultransonic inspection, any surface roughness that would interfere with that inspection shall be removed. All cylinders shall be drained of fluids.

\section{D.4 Ultrasonic Inspection}

\section{D.4.1 General}

This section is based on the ultrasonic inspection techniques described in Annex B of ISO 98091. Other inspection techniques may be used, provided that they have been demonstrated to be suitable for the detection of the reference notches.

\section{D.4.2 Requirements}

The ultrasonic testing equipment shall be capable of detecting, at a minimum, the reference standards described in Clauses D.4.3.2 and D.4.5.2. The equipment shall be serviced regularly in accordance with the manufacturer's operating instructions to ensure that its accuracy is maintained. Inspection records and approval certificates for the equipment shall be maintained.

The operation of the testing shall be performed by trained personnel and supervised by qualified and experienced personnel certified to Level 2 of ISO 9712.

The outer and inner surfaces of any cylinder that is to be tested ultrasonically shall be in a condition suitable for an accurate and reproducible test.

For flaw detection, the pulse echo system shall be used. For thickness measurement, either the resonance method or the pulse echo system shall be used. Either contact or immersion testing techniques shall be used. A coupling method that ensures adequate transmission of ultrasonic energy between the testing probe and the cylinder shall be used.

\section{D.4.3 Flaw Detection in the Cylinder Sidewall}

\section{D.4.3.1 Procedure}

The cylinder to be inspected and the search unit shall have a rotating motion and translation relative to one another such that a helical scan of the cylinder will be described. The velocity of rotation and translation shall be constant within $\pm 10 \%$. The pitch of the helix shall be less than the width covered by the probe (at least $10 \%$ overlapping shall be guaranteed) and be related to the effective beam width in such a way as to ensure $100 \%$ coverage at the velocity of rotation and translation used during the calibration procedure.

An alternative scanning method in which the scanning or relative movement of the probes and the work piece is longitudinal may be used for transverse defect detection. The sweeping motion shall be such as to ensure $100 \%$ coverage at the velocity of rotation and translation used during the calibration procedure.

An alternative scanning method in which the scanning or relative movement of the probes and the work piece is longitudinal may be used for transverse defect detection. The sweeping motion shall be such as to ensure a $100 \%$ surface coverage with about $10 \%$ overlapping of the sweeps.

The cylinder wall shall be tested for longitudinal defects with the ultrasonic energy transmitted in both circumferential directions and for transverse defects in both longitudinal directions.

The effectiveness of the equipment shall be periodically checked by submitting a reference standard to the test procedure. At a minimum, this check shall be carried out at the beginning 
(c) Canadian Standards Association

and end of each shift. If during this check the appropriate reference notch is not detected, all cylinders tested subsequent to the last acceptance check shall be retested after the equipment has been reset.

\section{D.4.3.2 Sidewall Reference Standard}

A reference standard of convenient length shall be prepared from a cylinder sidewall of similar diameter and wall thickness range, and made of material with the same acoustic characteristics and surface finish as the cylinder to be inspected. The reference standard shall be free from discontinuities that may interfere with the detection of the reference notches.

Reference notches, both longitudinal and transverse, shall be machined on the outer and inner sidewall surface of the standards. The notches shall be separated in such a way that each notch can be clearly identified.

The dimensions and shape of notches are crucially important for the adjustment of the equipment and shall conform to the following requirements:

a) The length of a notch shall not be greater than $25 \mathrm{~mm}$.

b) The width of a notch shall not be greater than $2 \mathrm{~mm}$.

c) The depth of a notch shall be $5 \% \pm 0.75 \%$ of the wall thickness over the full length of the notch. Runouts at each end shall be permitted.

d) A notch shall be sharp-edged at its intersection with the surface of the cylinder wall. The cross-section of a notch shall be rectangular except where spark-erosion machining methods are employed, in which case the bottom of the notch shall be rounded.

e) The shape and dimensions of a notch shall be demonstrated by an appropriate method.

\section{D.4.3.3 Calibration of Equipment}

The equipment shall be adjusted to provide clearly identifiable indications from inner and outer reference notches in accordance with the sidewall reference standard described in Clause D.4.3.2. The amplitude of the indications shall be as close to identical as possible. The indication of smallest amplitude shall be used as the rejection level and for the setting of visual, audible recording, or sorting devices. The equipment shall be calibrated with the reference standard or probe, or both, moving in the same manner, direction, and speed as will be used during the inspection of the cylinder. All visual, audible, recording, and sorting devices shall operate satisfactorily at the test speed.

\section{D.4.4 Wall Thickness Measurement}

One hundred per cent of the cylindrical part shall be examined to ensure that the wall thickness is not less than the minimum design thickness and that the reduction in wall thickness is less than $5 \%$ of the typical wall thickness of the cylinder being examined. The sidewall reference standard specified in Clause D.4.3.2 shall be sued to calibrate the thickness measurement.

\section{D.4.5 Flaw Detection in the Cylinder Ends}

\section{D.4.5.1 Procedure}

The entire surface of the ends shall be examined using a $45^{\circ}$ angle probe.

The scans shall be made as follows:

a) with the probe parallel to the longitudinal axis; and

b) with the probe at right angles to the longitudinal axis. 
(c) Canadian Standards Association

Each scan shall be carried out with the probe pointing in one direction and then repeated with the probe reversed. In addition, a scan shall be carried out at the root of the neck with the probe held at an angle of $45^{\circ}$ to the longitudinal axis of the cylinder and then repeated with the probe turned to $90^{\circ}$. The scans shall be made using probes with maximum dimensions of 10 to $20 \mathrm{~mm}(0.4$ to $0.8 \mathrm{in})$, the smaller probes being used to scan the root of the neck.

Scans shall begin or terminate at points $50 \mathrm{~mm}$ ( 2 in) along the parallel part of the cylinder, and each individual scan shall overlap the pervious scan by $25 \%$.

\section{D.4.5.2 End Reference Standards}

A reference standard of convenient length shall be prepared from a cylinder head end and a cylinder base end of similar diameter and wall thickness range, and made of material with the same acoustic characteristics and surface finish as the cylinder to be inspected. The reference standard shall be free from discontinuities that may interfere with the detection of the reference notches.

Reference notches, both longitudinal and transverse, shall be machined on the outer and inner surfaces of the standard head end and base end. The notches shall be separated in such a way that each notch can be clearly indentified. At the head end, the notches shall be located in the neck curvature near the base of the threads. At the base end, the notches shall be located in the curved portion of the transition adjacent to the cylinder sidewall.

The dimensions and shape of notches are crucially important for the adjustment of the equipment and shall conform to the following requirements:

a) The length of a notch shall not be greater than $25 \mathrm{~mm}$.

b) The width of a notch shall not be greater than $1 \mathrm{~mm}$.

c) The depth of a notch shall be $10 \% \pm 0.75 \%$ of the wall thickness over the full length of the notch. Runouts at each end shall be permitted.

d) A notch shall be sharp-edged at its intersection with the surface of the cylinder wall. The cross-section of a notch shall be rectangular except where spark-erosion machining methods are employed, in which case the bottom of the notch shall be rounded.

e) The shape and dimensions of a notch shall be demonstrated by an appropriate method.

\section{D.4.6 Assessment of Results}

\section{D.4.6.1 General}

If surface defects are removed by grinding, the cylinders shall also be subjected to ultrasonic defect detection and thickness measurement after the grinding. Cylinders continuing to show defects at points of minimum design thickness shall be deemed not to comply with the requirements of this procedure.

\section{D.4.6.2 Acceptance Criteria for Steel Vehicle Cylinders}

The acceptance criterion for the sidewall of a cylinder that is designed in accordance with this Standard shall be as specified in Table D.1.

The acceptance criterion for the ends of a cylinder that is designed in accordance with this Standard shall be the absence of any defect indication that is equal to or greater than the end reference notches.

\section{D.4.6.4 Destruction of Cylinders That Fail the Examination}

Cylinders that fail either the sidewall or the end inspection shall be destroyed. 


\section{D.5 Protective Coating}

\section{D.5.1 Recoating}

Cylinders that are designed in accordance with this Standard for vehicle use shall be recoated to meet the requirements of Section 2.6 of this Standard. Stamping marks shall also be coated.

\section{D.5.2 Visibility of Markings}

Requalifying agencies shall ensure that all markings are visible after a cylinder has been recoated and before the cylinder is shipped.

\section{D.6 Stamping or Labelling Procedure}

\section{D.6.1 Unique Marking}

A requalified cylinder shall be marked with unique marking that will identify the agency approved or recognised by the authority having jurisdiction over the cylinder designs covered by this procedure for the requalification of cylinders. This marking shall be registered with the local authority having jurisdiction.

\section{D.6.2 Length of Requalification Period}

Cylinders that have been requalified shall be marked with a retest due date that is no more than 15 years from the date of inspection.

The service life of steel vehicle cylinders shall not exceed 30 years from the date of manufacture.

\section{D.6.3 Use after Requalification}

If not previously marked, a requalified cylinder shall be marked CNG ONLY. The lettering shall be at least $6 \mathrm{~mm}(0.24 \mathrm{in})$ high.

\section{D.6.4 Expiry Date - Steel Vehicle Cylinders}

Steel vehicle cylinders that have been requalified shall be labelled with the words DO NOT USE AFTER followed by the year of expiry (at most, 15 years from the date of ultrasonic inspection or 30 years from the date of manufacture, whichever comes first). The lettering shall be at least 6 $\mathrm{mm}(0.24 \mathrm{in})$ high. The following is an example:

\section{DO NOT USE AFTER 2018}

(inspector's sample)

\section{D.7 Storage and Shipment Procedure}

For a cylinder where the valve has been removed, the internal surfaces of the cylinder shall be sprayed with a vapour-phase corrosion inhibitor (or equivalent). The cylinder shall then be sealed to atmosphere for storage or transport.

\section{D.8 Documentation}

A record of the inspection and stamping shall be made for each requalified cylinder. Records shall be kept for at least 15 years by the agency identified in Clause D.6.1. One copy of the completed form for each cylinder shall be provided to the cylinder owner. 


\section{D.9 Quality Control System}

The quality control system for the inspection of cylinders used by the agency identified in Clause D.6.1 shall comply with the requirements of Section 15 of this Standard.

\section{Table D.1}

\section{Acceptance Criteria for Steel Vehicle Cylinders}

(See Clause D.4.6.2)

\begin{tabular}{ll}
\hline $\begin{array}{l}\text { Crack depth (d) expressed as a } \% \text { of } \\
\text { wall thickness }\end{array}$ & Permissible crack length \\
\hline$D \leq 4 \%$ & No limit \\
$4 \%<D \leq 5 \%$ & $8 \mathrm{~mm}$ or less \\
$5 \%<D \leq 7 \%$ & $6 \mathrm{~mm}$ or less \\
$7 \%<D \leq 10 \%$ & $5 \mathrm{~mm}$ or less \\
$10 \%<D \leq 15 \%$ & $4 \mathrm{~mm}$ or less \\
$D>15 \%$ & Not acceptable \\
\hline
\end{tabular}




\title{
Appendix P - \\ Some Things to be Learned from the "Other" Compressed Gas Fuel System
}

\author{
Henry E. Seiff \\ Clean Vehicle Education Foundation
}

Copyright (C) 2008 CVEF

\begin{abstract}
Compressed natural gas vehicles were first commercialized after World War II in Italy. There are now seven million CNG vehicles on the road worldwide. The first US CNG vehicle "incident" in our files dates to 1984. "Those who cannot learn from history are doomed to repeat it" (1), so this paper will explore a few of the things to be learned from CNG vehicle history that can help assure the safety of compressed hydrogen tanks and fuel systems.
\end{abstract}

\section{A LITTLE BACKGROUND INFORMATION}

Compressed natural gas as a motor vehicle fuel has been around for a long time.

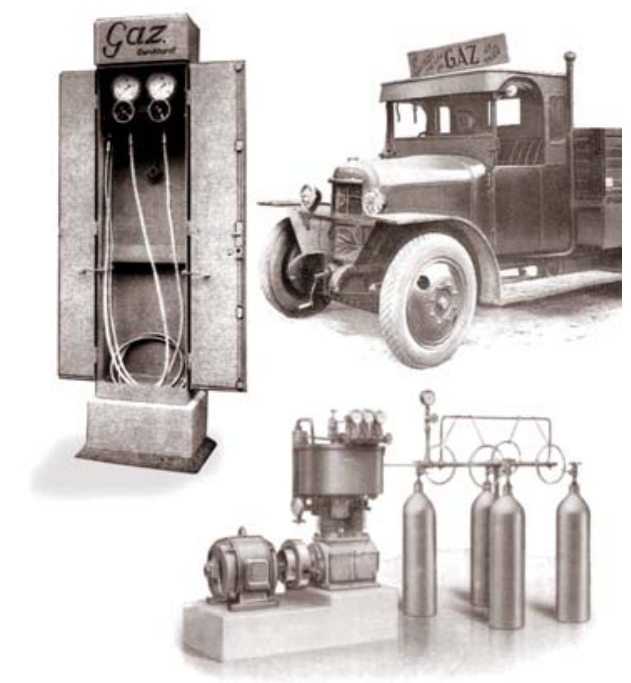

Figure 1: Historic CNG vehicle and equipment (2)

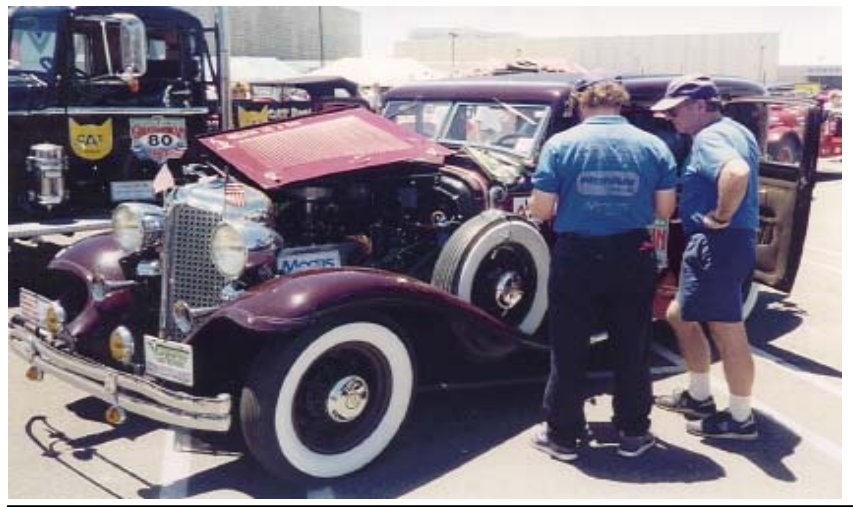

Figure 2: 1932 Chrysler "Ironsides" powered by a Mogas Natural Gas System (3)

Although low in number in the United States, worldwide there are seven million natural gas vehicles (NGVs) on the road today and a target of 50 million for 2020 (4). Natural gas vehicles offer some major advantages, such as:

- $\quad$ they use zero petroleum

- $\quad$ they are inherently cleaner burning than gasoline or diesel

- they produce around $25 \%$ less Greenhouse gas

- the fuel is less expensive on an energy equivalent basis

and some disadvantages:

- the fuel system costs more to produce

- the fuel system takes up more space and weighs more for the same driving range

- there is a limited natural gas fueling infrastructure in place 
Interestingly, the safety record of natural gas vehicles has proved to be as good as, if not better, than petroleum-powered vehicles. (5)

It is also interesting, but not surprising, that the advantages and disadvantages of natural gas (which is almost all methane) as a vehicle fuel are similar to those of hydrogen. Four-fifths of the atoms in a natural gas molecule are

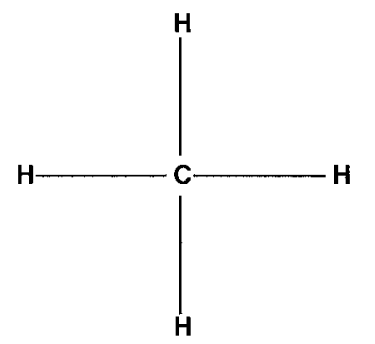

Figure 3: Natural gas molecule is $4 / 5$ hydrogen.

hydrogen and vehicular natural gas is most often transported in high-pressure cylinders, just like hydrogen.

So, given that "those who cannot learn from history are doomed to repeat it," what can be learned from 60 or more years of experience with natural gas vehicles, particularly, what can be learned from our experience with compressed natural gas fuel systems, which are likely to be very similar to compressed hydrogen fuel systems if and when hydrogen-powered vehicles become popular?

\section{A VECHICULAR COMPRESSED GAS FUEL SYSTEM PRIMER}

This paper concentrates on the high-pressure cylinder and related items, particularly the cylinder and the pressure relief device normally used to protect against cylinder rupture in a fire.

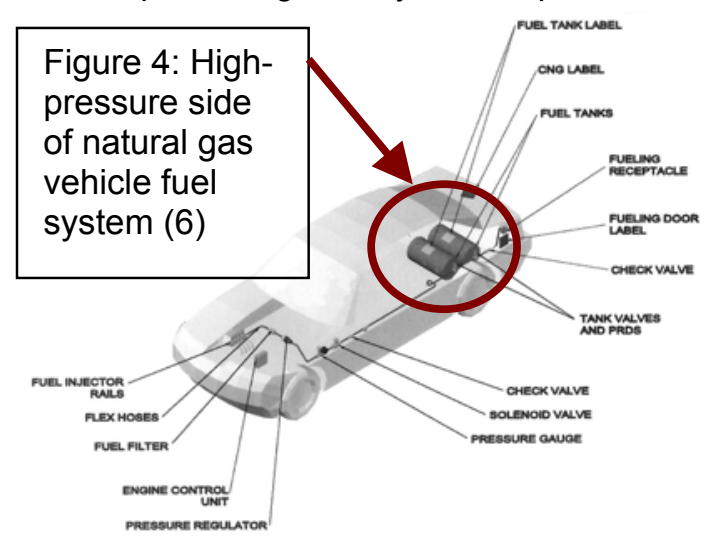

The safety of CNG cylinders is assured by Federal Motor Vehicle Safety Standard 304 (7), as well as ANSI/CSA NGV2 (8), the industry standard generally adhered to in the US, which is somewhat more stringent than FMVSS 304. Hydrogen cylinder safety standards are being written by both CSA and SAE at this time.

Four types of cylinders are normally used for vehicular compressed gas fuel (8):

\section{Type 1 - Metal}

Type 2 - Resin impregnated continuous filament with metal liner with a minimum burst pressure of $125 \%$ of service pressure. The container may be either hoop-wrapped or full-wrapped.

Type 3 - Resin impregnated continuous filament with metal liner. The container may be either hoop-wrapped or full-wrapped.

Type 4 - Resin impregnated continuous filament with a non-metallic liner.

Type 1 steel cylinders are the least expensive and the heaviest. They are generally used on CNG vehicles in the developing world. Type 3 and 4 cylinders, wrapped with fiberglass and/or carbon filament, are the lightest and most expensive cylinders. Because of their relatively light weight (1/4 -1/2 the weight of an equivalent steel type 1 cylinder), they are generally used in the US on OEM natural gas vehicles, such as transit buses and are the type of cylinders normally considered for vehicles operating on compressed hydrogen.

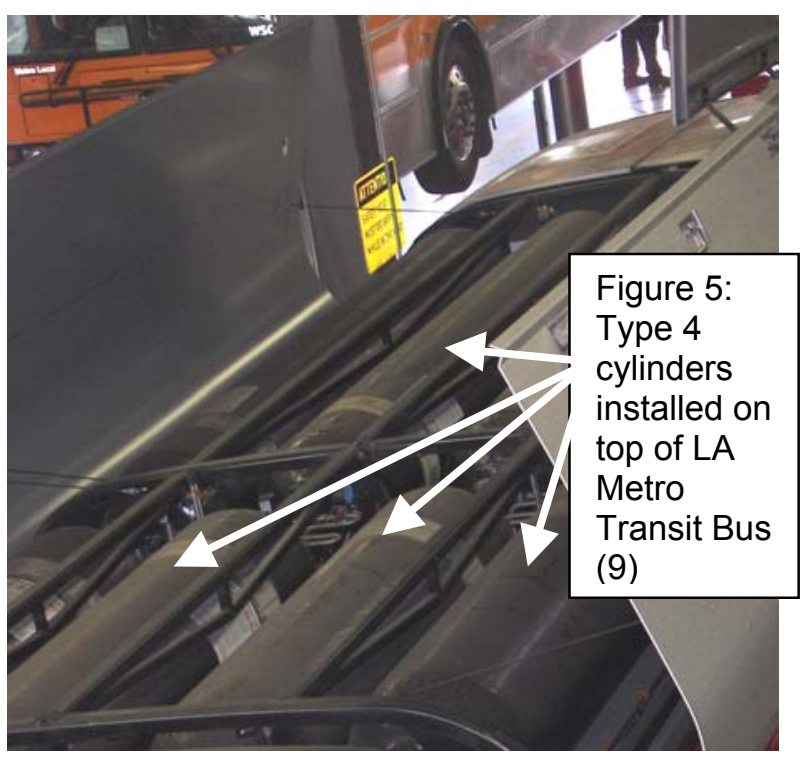


The service pressure of vehicular CNG cylinders worldwide is normally 200 Bar (3,000 psi), although most US CNG vehicles operate at 250 Bar (3,600 psi). Since hydrogen must be compressed to much higher pressures to provide a sufficient amount of energy in a reasonable space, pressures of 350 - 700 Bar $(5,000-10,000 \mathrm{psi})$ are being considered.

The standards under which CNG cylinders are built assure that they will withstand at least 2.25 times service pressure and "leak before burst" (LBB) if punctured or cycled. In particular, the standards require that the cylinder shall not rupture after having been "penetrated by an armor piercing bullet with a diameter of 7.62 mm" (\$18.11, ANSI/CSA NGV2-2000) or when subjected to a minimum of 11,250 cycles at $125 \%$ of service pressure ( $\$ 12.5 .2 .1)$. They also undergo a number of other tests to assure safety, such as an accelerated stress rupture test, a drop test, and exposure to various corrosive environmental fluids.

To avoid potential rupture, cylinders must be protected with pressure relief devices. Despite their name, these PRDs must be designed to protect against fire, not excessive pressure (although some PRDs protect against both). The logic behind this is two-fold:

1- The fueling dispenser has at least two levels of overpressure protection, so it is relied on to assure that the cylinder is not over pressurized during fueling. This leaves a vehicle fire as the only reasonable way a cylinder might experience excessive pressure.

2- When exposed to a fire, the gas in Type 1 (all metal) cylinders could be expected to increase in pressure as it heats up and vent through a pressure relief valve before a cylinder ruptured. However Types 2-4, especially Types 3 and 4 cylinders, would likely have their strength compromised by the fire before the pressure increased enough to vent through a pressure relief valve. Therefore they must be protected by a temperature sensitive PRD.

Both the government (FMVSS 304) and industry (ANSI/CSA NGV2) CNG cylinder standards provide for a bonfire test to assure that the PRD operates properly. The industry ANSI/CSA PRD1 (10) standard sets other safety requirements of $P R D$ s used on $C N G$ vehicles. $A$ hydrogen version of PRD1 (HPRD1) is currently under development by the industry.

In addition to these design and test requirements to assure natural gas vehicle safety, cylinders and their accompanying fuel systems should undergo a detailed safety inspection at least every three years or 36,000

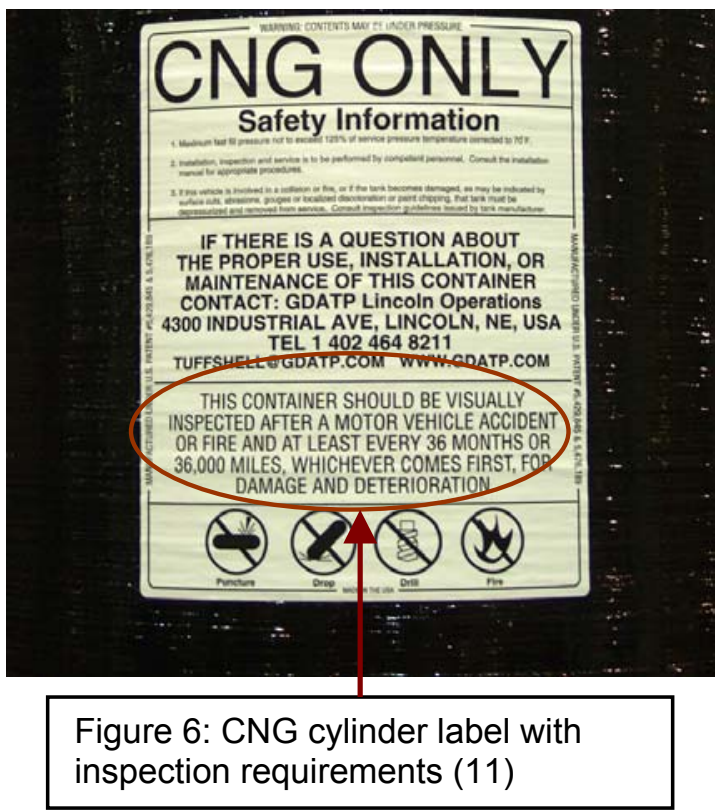

miles or when they have been involved in collisions, accidents, fires or other damage (ANSI/NGV2 $\$ \S 4.1 .4 \quad \& \quad 4.1 .5) \quad$ Specific inspection procedures are provided in the Compressed Gas Association's pamphlet C-6.4, supplemented by the cylinder manufacturer's own inspection instructions. There are a number of ways of providing the "qualified" inspectors required by CGA C-6.4, the best known being the CSA inspector qualification test (12), which now provides over 500 certified inspectors nationwide.

As some of the discussion above indicates, the natural gas vehicle industry has been extremely concerned about safety in the US. In fact, there has been only one fatality in the US caused by the natural gas fuel system of a vehicle! So there is a lot of safety information from the manufacture and operation of those vehicles, which may be applicable to hydrogen-powered vehicles. 


\section{NATURAL "INCIDENTS" \\ GAS}

The Clean Vehicle Education Foundation and its predecessor organization, the Natural Gas Vehicle Coalition, run a voluntary NGV incident reporting program in order to learn from incidents involving NGVs. This has allowed us to improve NGV codes and standards to assure higher levels of vehicle safety over the years. To date we have details of 76 incidents since 1984 , primarily in the US, as well as information on 44 cylinder failures worldwide (13) since 1976 , plus a number of other overseas incidents (Contact the author for a summary list or details on these incidents). Learning from domestic and overseas incidents and incorporating the information in US NGV codes and standards helps ensure this enviable safety record.

\section{SOME THINGS THE NGV INDUSTRY HAS LEARNED WHICH APPLY TO HYDROGEN VEHICLES}

Some of the issues which the natural gas vehicle industry has seen which the hydrogen vehicle industry can learn from follow.

VEHICLE CYLINDERS CAN GET OVER PRESSURIZED AND RUPTURE - An obvious concern of any industry dealing with highpressure compressed gas is over pressurization of a gas cylinder (or any other part of the system) which could lead to cylinder failure, or in the worst case, cylinder rupture.

The NGV industry assigns the responsibility to avoid over pressurization to the dispenser at the fueling facility. The vehicle system has no protection against over pressurization as such (the Pressure Relief Device on the vehicle is required to protect against fire, although some devices may also protect against over pressurization).

NFPA 52 (Vehicular Fuel Systems Code) (14) (§8.6.3) requires that "An overpressure protection device...shall be installed in the fueling transfer system to prevent overpressure in the vehicle."

ANSI/NGV4.1 (NGV Dispenser Systems) (15) requires two overpressure protection systems. Section 1.11.4 on overfill protection requires: "Each dispensing system shall be fitted with a safety valve set to relieve pressure no more than..." $125 \%$ of service pressure "...to prevent cylinder over pressurization." And \$1.4.2 requires: "A pressure relief valve shall be located downstream of the overfill protection system to prevent over-pressurization of the vehicle storage vessels."

And yet there have been cases where the dispenser protection has failed to keep vehicle cylinders from being over pressurized and rupturing.

In 2000 one cylinder mounted in the bed of a pickup truck ruptured, apparently from over pressurization. It is speculated that multiple failures of pressure regulating and relief systems allowed the dispensing of 6000 psi gas, into 2400 psi cylinders. The force of the rupture propelled the cylinder through the roof of an adjoining bowling alley. Luckily the bowling alley was unoccupied at the time so there were no injuries from the incident.

Also in 2000, a vehicle refueling appliance (a small dispenser) failed, allowing the over pressurization and rupture of a CNG cylinder on a delivery truck being fueled in Montreal, Canada. There were also no injuries from this incident.

Lesson for compressed hydrogen vehicles: Consider whether vehicle tanks should also provide protection against over pressurization.

PHYSICAL OR CHEMICAL ABUSE CAN DAMAGE CYLINDERS - CNG cylinders built to ANSI/NGV2 standards must pass tests to assure they can stand up to physical and chemical abuse. Section 18.8 requires withstanding a $1.83 \mathrm{~m}$ (6 foot) drop test such as might happen if a cylinder fell from a forklift. Section 18.4 requires an "environmental test" in which the cylinder is first impacted, then exposed to five different types of corrosive fluid, including sulfuric acid. This last test was added when some cylinders failed in service after battery acid in a truck bed leaked onto cylinders and failed the fiberglass overwrap from stress corrosion cracking.

The environmental test was added to the NGV2 standard in 1998 but some older cylinders are still in use. The first US fatal accident attributed to the CNG fueling system happened in May 2007, when an older add-on tank on a 
SuperShuttle van ruptured during fueling. It appears the failure was due to acid exposure on the tank during a previous accident.

Since the 1995 edition, NFPA 52 (§6.3.2.1) has required that "fuel supply containers shall be protected with a means to prevent damage that can occur due to road hazards, loading, unloading, direct sunlight, exhaust heat, and vehicle use including accidental cargo leakage." However in August 1996 a cylinder on a Los Angeles transit bus, apparently built to an earlier standard, ruptured due to physical damage. "Since the rupture all of the buses have been equipped with shields to protect the cylinders (which) they did not have before the rupture" (NGV News, August 1997).

In 1994 and 1996, prior to the environmental test requirement in NGV2, two cylinders ruptured from exposure to battery acid. And, as mentioned above, another older cylinder recently failed from this cause and killed the man fueling the vehicle in May of 2007.

Lesson for compressed hydrogen vehicles: Cylinders must be designed to protect against corrosive agents, road debris, and other types of foreseeable damage. Alternatively cylinders must be mounted in the vehicle to avoid these types of potential damage.

CYLINDERS/FUEL SYSTEMS NEED INSPECTION - In September 2003, in Scarborough, Ontario (a suburb of Toronto) a cylinder ruptured during fueling of a Dodge Ram van which had been converted to CNG in 1994. "Apparently the cylinder failed during fueling at substantially under its rated 3000 psi (200 Bar). The driver said he had smelled gas for a long time but hadn't gotten around to checking the source. It was reported that there was massive external corrosion on the tank and there was no evidence of it having been inspected." (16)

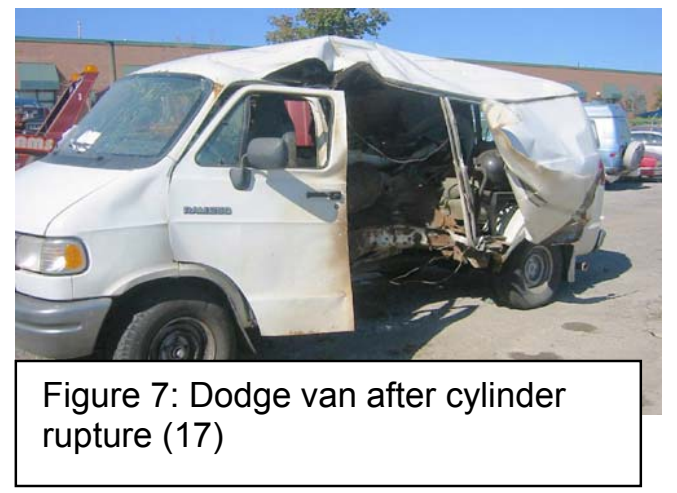

As discussed above, the natural gas vehicle industry advises cylinder users to routinely have a detailed visual inspection at least every 36 months and FMVSS 304 requires the cylinder label to state that "This container should be visually inspected after a motor vehicle accident or fire and at least every 36 months or 36,000 miles, whichever comes first, for damage or deterioration." On the Dodge van, the Canadian cylinder registration required an inspection every three years. (18)

A detailed visual inspection, conducted per the Compressed Gas Association pamphlet C-6.4 (Methods for External Visual Inspection of Natural Gas Vehicle Fuel Containers and Their Installations) (19) referenced in the industry container standard, ANSI/CSA NGV2, includes inspection of much of the CNG fuel system, not just the fuel container. Therefore these inspections find gas leaks, PRD or fuel line damage, and many other potential problems before they lead to an incident. Periodic CNG fuel system inspections are no different from brake, headlight or tire inspections in their ability to help ensure safe vehicle operation.

Lesson for compressed hydrogen vehicles: Periodic fuel system safety inspections can help ensure safe vehicle operation.

PRDs SOMETIMES LEAK - PRDs (pressure relief devices) are routinely used with CNG containers to protect against rupture during a fire. They sense high temperature and vent the high-pressure gas before the fire can compromise the tank strength and allow it to rupture. Vehicular hydrogen tanks, which generally operate at a higher pressure than CNG, also use PRDs.

Two types of problems can occur with PRDs in service. A type 1 failure, where the PRD fails to properly vent the gas during a fire, is likely to be more serious, potentially allowing the tank to rupture. A type 2 failure, the unintended venting of the PRD during normal vehicle operation, can also cause serious problems.

Normally a type 2 failure causes only the loss of the gaseous fuel into the atmosphere, and is an inconvenience to the operator. But a leaking PRD can also occasionally lead to a serious fire as it did in an Akron, Ohio bus garage in 1999. In this case, there were two preceding leaks, 
one two weeks before and one seven hours before the leak which led to the fire (20).

Although it is not known exactly why the PRDs leaked in Akron, the cause of a similar incident about the same time in State College, PA is known. In that case and in others, moisture got into the PRD vent tube, froze and, as it expanded, damaged the PRD. Although vent caps are normally used to prevent this, and some CNG bus users drill "weep holes" to allow water to drip out, this problem continues, although at a much decreased rate.

Lesson for compressed hydrogen vehicles: With the potential for high volumes of compressed hydrogen vehicles on the highways, type 2 failures, where PRDs vent unintentionally, must be eliminated or at least minimized.

PRDS DON'T ALWAYS PROTECT AGAINST FIRES, ESPECIALLY LOCALIZED FIRES ANSI/CSA PRD1 (§1.2) contains a warning that "Pressure relief devices may not prevent rupture of a container under all conditions of fire exposure. When the heat transferred to the container is localized, intensive, and remote to the relief device, or where the fire builds extremely rapidly, such as in an explosion, and is of very high intensity, the container may weaken sufficiently to rupture before the relief device operates, or while it is operating." This, or a similar warning, will likely be carried over to the standard for compressed hydrogen PRDs.

In September 2002 a CNG cylinder on a Ford Crown Victoria police vehicle awaiting decommissioning "exploded during a fire" in a holding lot. "It was concluded that a direct flame (from the interior of the vehicle) onto the cylinder (located behind the rear seat in the car) compromised the hoop strength of the natural gas cylinder, thus allowing the cylinder to fail prior to the PRD releasing at its designed temperature." (21)

On March 26, 2007, an arsonist torched 12 vehicles in a Seattle, WA city government holding lot. All the vehicles were damaged or destroyed including a CNG Honda GX, whose fuel tank exploded in the fire. The tank was apparently compromised by fire moving from the back seat area onto the center of the cylinder, which was protected by a PRD at one end.

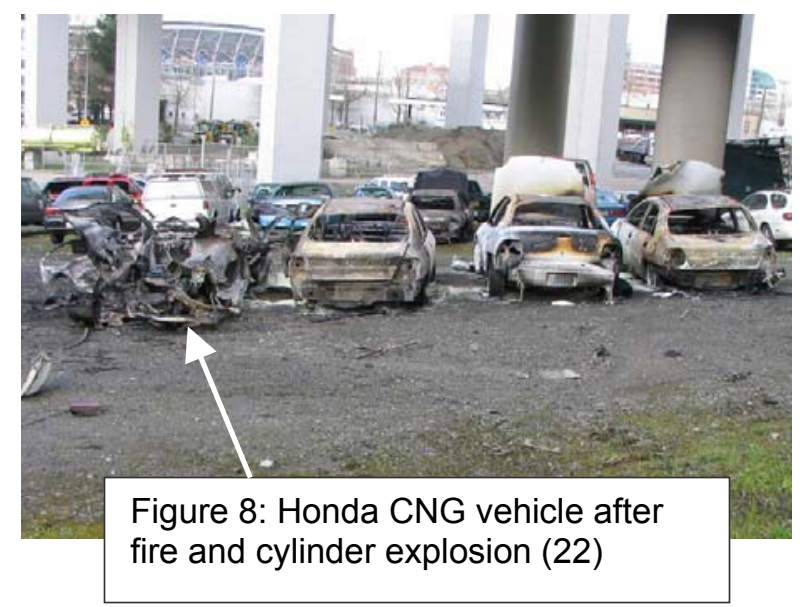

Lesson for compressed hydrogen vehicles: Although PRDs normally successfully protect CNG cylinders from explosion in vehicle fires, there are cases where present designs may not protect from localized fires. This could become a more serious concern if high volumes of compressed hydrogen vehicles on the highways using Types 2, 3, and 4 cylinders, are not protected by more advanced fire-sensing systems.

FIBER-WRAPPED CYLINDERS ARE EXPENSIVE: - A major expense for compressed natural gas or compressed hydrogen vehicles is the pressure vessels or cylinders used to hold the fuel. A ball-park estimate of the cost of various types of eight gasoline-gallon equivalent (gge) 250 Bar CNG cylinders is (23):

Type 1 - $\$ 375$

Type 2 - $\$ 580$

Type 3 - \$1022

Type 4 - $\$ 1022$

For compressed hydrogen far higher pressures and/or larger cylinders would be needed to hold an equal amount of fuel energy. This may be balanced against expected high efficiencies of fuel-cell vehicles, however.

Although Type 1 all steel cylinders are often used for CNG vehicles in the developing world, some consider them too heavy for use on OEM NGVs or hydrogen vehicles in the US. However, all of cylinders have been used on OEM CNG vehicles, but Types 3 or 4 are generally talked about for hydrogen vehicles, probably because of their lower weight, especially when 350 to 700 Bar pressures are considered.

The relatively high cost of compressed gas cylinders has to do with: 
- High costs of fiber overwrap, especially carbon fiber

- low (compared to normal automotive production numbers) production volumes

- slow formation times for cylinder liners

- $\quad$ slow and expensive fiber winding methods.

Lesson for compressed hydrogen vehicles: Lower cost manufacturing processes and materials must be found to substantially lower the cost of cylinders for hydrogen automotive vehicles to be competitive in the marketplace.

\begin{abstract}
HIGH-PRESSURE CYLINDERS HAVE A LIMITED LIFE AND NEED PERIODIC SAFETY INSPECTION - We are used to seeing high pressure cylinders being used for welding, or holding medical gases in hospitals or even dentists' offices. These are normally "DOT" cylinders (built to Department of Transportation regulations) which require periodic hydrostatic testing for safety. They are generally the all steel type 1 cylinders, which are inexpensive, extremely heavy and almost "bullet proof." The lighter-weight, more expensive and higher technology type 3 or 4 cylinders likely to be used on hydrogen-powered vehicles will have a limited life and likely also require periodic safety inspection.
\end{abstract}

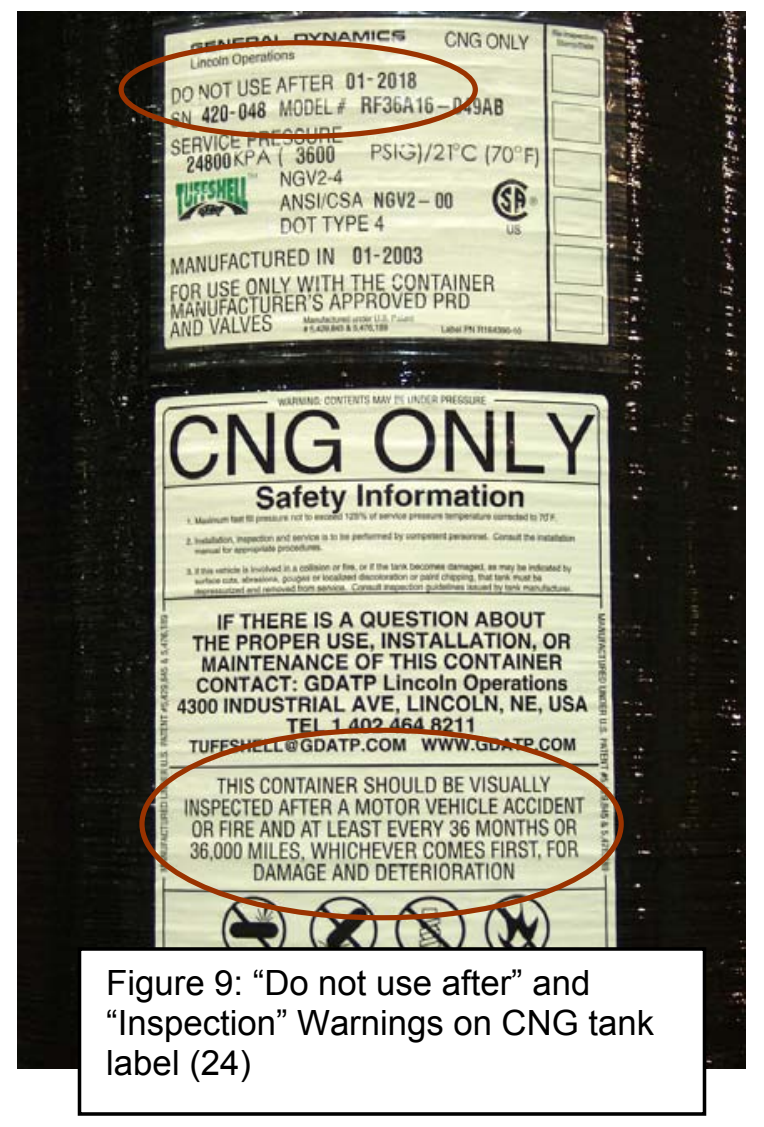

Compressed natural gas cylinders in the US carry a notice that they should be inspected after an accident or fire and every 36 months or 36 thousand miles, whichever comes first. They also have a limited life, and carry an expiration date, after which they should be replaced.

Two potential problems are the possibility of moving cylinders, pressure relief devices, and other fuel system components from one vehicle to another and enforcing the "end of life" date on the cylinders. Although both those in the automotive industry working on CNG and hydrogen vehicles are strongly opposed to allowing components from one vehicle to be moved to another vehicle (such as using components on a scrapped vehicle to repair another damaged vehicle), there is no clear way to avoid this potential danger. The concern is that "donor" components used in this manner might not be safe to use on the "recipient" vehicle.

State periodic motor vehicle inspections (PMVI) might be used to ensure that these transplants never occur, but at present only 18 states, the District of Columbia and Puerto Rico have PMVI in place and the number is decreasing. Another potential solution is to provide for free exchange of cylinders and other components when inspection is needed. Such a system is in place in Italy where fuel tax money is used to pay for replacement of cylinders at mandatory inspection periods.

Lesson for compressed hydrogen vehicles: - A way must be found to either make compressed hydrogen cylinders and fuel systems safe for the life of the vehicle, or to ensure they are inspected periodically to ensure improper components are not "transplanted" and that the system remains safe.

\section{REFERENCES}

(1) George Santayana, The Life of Reason, Volume I, Reason in Common Sense, (Wikiquote, http://en.wikiquote.org/wiki/ George_Santayana)

(2) European Natural Gas Vehicle Association (www.engva.org), private communication

(3) http://www.mogassales.com/Corporate/ ironside.htm 
(4) Top ten countries for NGVs (International Association for Natural Gas Vehicles, www.iangv.org)

\begin{tabular}{|c|c|c|}
\hline Position & Country & Number of NG Vel \\
\hline 1 & Argentina & $1,650,000$ \\
\hline 2 & Pakistan & $1,550,000$ \\
\hline 3 & Brazil & $1,425,513$ \\
\hline 4 & Italy & 432,900 \\
\hline 5 & India & 334,820 \\
\hline 6 & Iran & 263,662 \\
\hline 7 & Columbia & 203,292 \\
\hline 8 & USA & 146,876 \\
\hline 9 & China & 127,120 \\
\hline 10 & Ukraine & 100,00 \\
\hline
\end{tabular}

(5) Clean Vehicle Education Foundation Technology Committee Bulletin, "How Safe are Natural Gas Vehicles?", http://www.cleanvehicle.org/committee/technical/ PDFs/Web-TC-TechBul2-Safety.pdf

(6) Schematic done for CVEF by Powertech Labs, Surrey, British Columbia, Canada. www.powertechlabs.com.

(7) 49 CFR 571.304 (Compressed Natural Gas Fuel Container Integrity), available from http://www.access.gpo.gov/nara/cfr/cfr-tablesearch.html

(8) ANSI/CSA NGV2-2000 (Basic Requirements for Compressed Natural Gas Vehicle Fuel Containers) available from CSA, www.csaamerica.org

(9) Photo by author.

(10) ANSI/CSA PRD1 (Basic Requirements for Pressure Relief Devices for Natural Gas Vehicle Fuel Containers) available from CSA, www.csaamerica.org

(11) Photo courtesy of Lincoln Composites, Lincoln, NE, www.lincolncomposites.com.

(12) www.csa-america.org/advisory_service/ ngv_certification/

(13) "Known In-Service Failures of NGV Cylinders (Since 1976)," Powertech Labs, Inc., Surrey, British Columbia, Canada. www.powertechlabs.com.
(14) National Fire Protection Association, NFPA 52 (Vehicular Fuel Systems Code) available from NFPA, www.nfpa.org

(15) ANSI/NGV4.1 (NGV Dispenser Systems) available from CSA, www.csa-america.org

(16) NGVCommunications, weekly member newsletter of the Natural Gas Vehicle Coalition, September 12, 2003.

(17) Case Study, Natural Gas Fuel Cylinder Failure, Leland Shields, www.vehiclefire investigation.com/hybridalternative/hybridalterna tive12b.html

(18) "Natural Gas Fuel Cylinder Failure on 03/09/03," fact sheet was prepared by Charonic Canada Inc. in conjunction with Powertech Labs Inc. and the Ontario Technical Standards and Safety Authority.

(19) CGA C-6.4 (Methods for External Visual Inspection of Natural Gas Vehicle Fuel Containers and Their Installations), available from the Compressed Gas Association, www.cganet.com

(20) Akron Beacon-Journal, December 30, 1999.

(21) NHTSA Office of Defects Investigation Recall web site. Recall campaign number 03V472000.

(22) Photo from "Firefighter Near Miss," City of Seattle Fire Department Operations Division, May 9, 2007.

(23) Private communications.

(24) Photo courtesy of Lincoln Composites, Lincoln, NE, www.lincolncomposites.com.

\section{CONTACT}

Hank Seiff, P.E.

Director of Technology

Clean Vehicle Education Foundation hseiff@cleanvehicle.org 1-703-534-6151 